\title{
Biomineralized collagen for bone regeneration
}

Citation for published version (APA):

De Melo Pereira, D. (2022). Biomineralized collagen for bone regeneration: Insights into cell-material interactions. [Doctoral Thesis, Maastricht University]. ProefschriftMaken. https://doi.org/10.26481/dis.20220124dp

Document status and date:

Published: 01/01/2022

DOI:

10.26481/dis.20220124dp

Document Version:

Publisher's PDF, also known as Version of record

\section{Please check the document version of this publication:}

- A submitted manuscript is the version of the article upon submission and before peer-review. There can be important differences between the submitted version and the official published version of record.

People interested in the research are advised to contact the author for the final version of the publication, or visit the DOI to the publisher's website.

- The final author version and the galley proof are versions of the publication after peer review.

- The final published version features the final layout of the paper including the volume, issue and page numbers.

Link to publication

\footnotetext{
General rights rights.

- You may freely distribute the URL identifying the publication in the public portal. please follow below link for the End User Agreement:

www.umlib.nl/taverne-license

Take down policy

If you believe that this document breaches copyright please contact us at:

repository@maastrichtuniversity.nl

providing details and we will investigate your claim.
}

Copyright and moral rights for the publications made accessible in the public portal are retained by the authors and/or other copyright owners and it is a condition of accessing publications that users recognise and abide by the legal requirements associated with these

- Users may download and print one copy of any publication from the public portal for the purpose of private study or research.

- You may not further distribute the material or use it for any profit-making activity or commercial gain

If the publication is distributed under the terms of Article $25 \mathrm{fa}$ of the Dutch Copyright Act, indicated by the "Taverne" license above, 


\section{Biomineralized collagen for bone regeneration Insights into cell-material interactions}

Daniel de Melo Pereira 
ISBN: 978-94-6423-516-6

Published December 2021

Copyright (C 2021 Daniel de Melo Pereira. All rights reserved.

Cover design by Danielle "a Talentosa" Ferreira Baptista

Printed by ProefschriftMaken

The work described in this thesis was carried out at the department of Instructive Biomaterials Engineering of the MERLN Institute for Technology-Inspired Regenerative Medicine at Maastricht University, the Netherlands.

This research has been made possible by the contribution of the Dutch Province of Limburg. The author would also like to acknowledge the contribution to the printing of this thesis from the Netherlands Society for Biomaterials (NBTE). 


\title{
Biomineralized collagen for bone regeneration Insights into cell-material interactions
}

\author{
Dissertation
}

To obtain the degree of Doctor at the Maastricht University, on the authority of the Rector Magnificus, Prof. Dr. Rianne M. Letschert in accordance with the decision of the Board of Deans, to be defended in public

on Monday the $24^{\text {th }}$ of January 2022, at 10:00 hours.

$$
\text { by }
$$

Daniel de Melo Pereira

Born on the $8^{\text {th }}$ of May 1989 in Figueira da Foz, Portugal 


\section{Promotor}

Prof. Dr. Pamela Habibović

\section{Assessment Committee}

Prof. Dr. Gunnar Seide (Chair)

Dr. Anat Akiva, Radboud UMC

Prof. Dr. Jenneke Klein-Nulend, Academic Center for Dentistry Amsterdam (ACTA), University of Amsterdam and VU Amsterdam

Prof. Dr. Martijn van Griensven

Dr. Vanessa LaPointe 


\section{Paranymphs}

Danielle F. Baptista

Jasia A. King 


\section{Table of Contents}

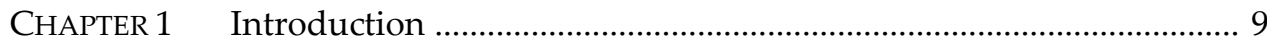

CHAPTER 2 Biomineralization-inspired materials design for bone regeneration

CHAPTER 3 Human osteoclast formation and resorptive function on biomineralized collagen 61

CHAPTER 4 Cobalt-containing calcium phosphate induces resorption of biomineralized collagen by human osteoclasts

CHAPTER 5 Proliferation and osteogenic differentiation of hMSCs on biomineralized collagen 121

CHAPTER 6 Behind the scenes: insights into technical details behind experimental studies

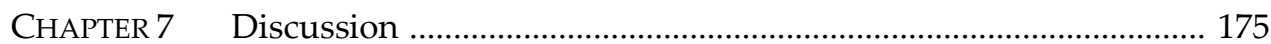

CHAPTER 8 Statement of impact ……………................................................... 191

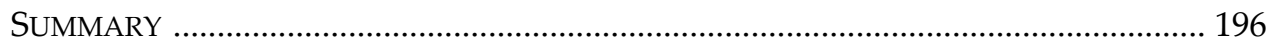

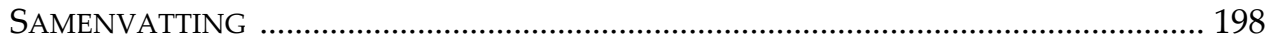

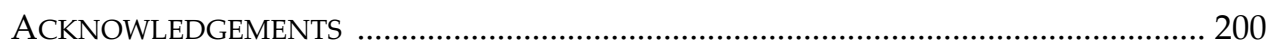

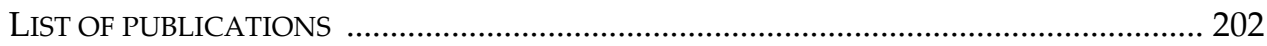


Chapter 1

Introduction 
Bone is the second most transplanted tissue worldwide, with around 1 million grafting procedures performed yearly, in Europe alone. ${ }^{[1]}$ This type of procedure is associated with a variety of clinical situations, for example complex fractures resulting from traumatic injuries, defects remaining after tumor removal, spinal fusion as treatment of degenerated intervertebral discs, and craniomaxillofacial surgery. ${ }^{[2-4]}$ In the majority of situations (estimated around $80 \%$ ), grafting procedures are carried out using either a patient's own bone tissue (autologous graft, considered the standard of care) or bone tissue from a donor (allogeneic graft). The share of treatments that do not include autologous or allogeneic grafts is smaller, but has been growing in past years, and is projected to continue increasing in the future. ${ }^{[5]}$ This is because autografts and allografts have inherent disadvantages, the most important being, respectively, the associated donor site morbidity, and risk of immune reaction. Both types of grafts are also limited in availability. Bone graft substitutes, being under continuous development, are expected to eventually have similar or even better clinical performance than the current standard of care, while overcoming their inherent drawbacks. A plethora of biomaterials is used as bone graft substitutes, but its largest segment consists of materials based on calcium phosphate $(\mathrm{CaP})$ or their composites, which have been in use for over half a century and proved to be closest to the goal of replacing autograft as the standard of care.[6] The biomimetic nature of CaP-based biomaterials, which closely resemble the composition of the inorganic component of bone, underpins their performance.

While CaPs are good mimics of the mineral component of bone, the intrinsic brittleness of (porous) ceramics limits their applicability to non-load bearing sites. To mimic both the chemical composition and the mechanical properties of bone, polymer-ceramic composites are of great interest. In this context, research efforts have been expended to develop biomineralized collagen, a composite of collagen and $\mathrm{CaP}$ in the form of hydroxyapatite that resides inside the collagen fibril (intrafibrillar mineral). The particular organization of the nano-sized hydroxyapatite crystals inside the collagen fibrils is a feature also found in natural bone tissue, and is considered a major contributor to its mechanical properties. ${ }^{[7]}$ Therefore, biomineralized collagen is a promising candidate material for bone graft substitute, as it not only provides a microenvironment that is chemically and structurally similar to the host tissue, but can also, through proper design and engineering, offer mechanical properties that would be similar to the native tissue. The state of the art in bone mimicking materials as well as our current knowledge of biomineralized collagen is reviewed in Chapter 2 of this thesis. 
To contribute to developments of synthetic biomimetic bone graft substitutes, the overall aim of this thesis is to improve our understanding of interactions between biomineralized collagen and cells residing in human bone. To provide a more specific context to the experimental chapters that follow, there are a few considerations regarding implantation of biomaterials in a bone environment, as well as bone physiology, which will be briefly introduced here.

One important aspect of bone graft substitutes is their osseointegration, or bonebonding property, defined as the ability to interface directly with bone tissue without formation of a soft fibrotic intermediate layer. Any fibrous capsule prevents the eventual replacement of the implanted material by natural bone tissue. ${ }^{[8]}$ The capacity to undergo creeping substitution, i.e., be gradually replaced by new bone tissue, is another important characteristic of bone graft substitutes. This entails two processes that should be coordinated, the removal of implant material, and the formation of bone tissue. ${ }^{[9]}$ Removal or degradation of a bone graft substitute can occur by three different mechanisms: physicochemical degradation (dissolution), fragmentation by mechanical forces, and cell-mediated processes such as small particle phagocytosis by macrophages or surface resorption by osteoclasts. ${ }^{[10]}$

In healthy bone tissue, there is a process that is somewhat analogous to creeping substitution, called bone remodeling. Bone remodeling is the main process by which bone tissue is renewed, and the structural stability of the skeleton maintained during the lifetime of an individual. Bone remodeling has two main consecutive stages. The first is removal of bone tissue, carried out by specialized cells named osteoclasts that differentiate from monocyte/macrophages from the hematopoietic cell lineage; the second is deposition of new bone tissue, carried out by osteoblasts that differentiate from multipotent mesenchymal stromal cells present in the bone marrow. These two processes are coupled, meaning that there is communication in various forms at different stages of the process, ensuring that the right amount of bone formation occurs at the right time following bone resorption. ${ }^{[11]}$

A biomaterial that is amenable to resorption by osteoclasts can be integrated in the resorption-deposition cycle of bone remodeling, and its resorption by osteoclasts can trigger the subsequent bone deposition by osteoblasts, thus leading to its replacement by native tissue. In this context, interactions with osteoclasts is an important aspect that characterizes the suitability of biomineralized collagen as a comprehensive synthetic bone graft substitute, and much of this thesis addresses this aspect. 
In Chapter 3, we investigate the capacity of human osteoclasts, derived from monocyte/macrophages from peripheral blood, to resorb biomineralized collagen membranes.

Based on the obtained results, in Chapter 4 we applied modifications to biomineralized collagen, adding extra mineral with incorporated cobalt ions, with the aim to enhance the resorptive activity of osteoclasts.

As mentioned above, new bone tissue is formed by osteoblasts, which differentiate from precursors originating from mesenchymal stromal cells. This process can be triggered by signaling occurring in the context of bone remodeling, as was already detailed, but it can also be initiated by other signals. Namely, the material used as bone graft substitute can elicit the recruitment of undifferentiated cells towards the osteoblast lineage, a process known as osteoinduction, and highly important in the context of bone regeneration. ${ }^{[12]}$ In Chapter 5, the interaction of biomineralized collagen with mesenchymal stromal cells is investigated, to understand to what extent this biomimetic material is able to trigger the osteogenic differentiation of these cells.

The experimental section of this thesis closes with Chapter 6, which is a collection of results obtained in the course of optimization of parameters related to both biomaterials and cells used in the thesis and justifies technical choices made in all other experimental chapters. Its purpose is to share valuable experimental information that did not make it to scientific publications resulting from this thesis, and yet may be useful for replication of experiments and follow-up research.

The General Discussion is given in Chapter 7, which places the main findings from this thesis against the backdrop of the existing knowledge. In this chapter, future perspectives concerning biomineralized collagen are discussed including suggestions for approaches to be used in further work, as well as a more general debate concerning biomimetic approaches to biomaterials engineering. 


\section{References}

[1] REBORNE (multiple authors), "Regenerating Bone defects using New biomedical Engineering approaches," can be found under https://cordis.europa.eu/project/1d/241879, 2015.

[2] S. K. Nandi, S. Roy, P. Mukherjee, B. Kundu, D. K. De, D. Basu, Indian J. Med. Res. 2010, 132, 15.

[3] S. D. Boden, Spine (Phila. Pa. 1976). 2002, 27, S26.

[4] A. Torroni, J. Oral Maxillofac. Surg. 2009, 67, 1121.

[5] Grand View Research (multiple authors), “Bone Graft And Substitutes Market Size, Share \& Trends Analysis Report By Material Type (Allograft, Synthetic), By Application (Craniomaxillofacial, Dental, Foot \& Ankle), By Region, And Segment Forecasts, 2021 - 2028," can be found under https://www.grandviewresearch.com/industry-analysis /bone-grafts-substitutesmarket, 2021.

[6] W. Habraken, P. Habibovic, M. Epple, M. Bohner, Mater. Today 2016, 19, 69.

[7] N. Reznikov, M. Bilton, L. Lari, M. M. Stevens, R. Kröger, Science (80-. ). 2018, 360, eaao2189.

[8] T. Rolvien, M. Barbeck, S. Wenisch, M. Amling, M. Krause, Int. J. Mol. Sci. 2018, 19, DOI 10.3390/ijms19102893.

[9] T. T. Roberts, A. J. Rosenbaum, Organogenesis 2012, 8, 114.

[10] Z. Sheikh, M. N. Abdallah, A. A. Hanafi, S. Misbahuddin, H. Rashid, M. Glogauer, Materials (Basel). 2015, 8, 7913.

[11] S. Takeshita, T. Fumoto, K. Matsuoka, K. Park, H. Aburatani, S. Kato, M. Ito, K. Ikeda, J. Clin. Invest. 2013, 123, 3914.

[12] M. Bohner, R. J. Miron, Mater. Today 2019, 22, 132. 


\section{Chapter 2}

\section{Biomineralization-inspired materials design for bone regeneration}

Daniel de Melo Pereira, Pamela Habibović

Published in Advanced Healthcare Materials, 2018, 1800700, 1-18. 


\begin{abstract}
Synthetic substitutes of bone grafts, such as calcium phosphate-based ceramics, have shown some good clinical successes in the regeneration of large bone defects, and are currently extensively used. In the past decade, the field of biomineralization has delivered important new fundamental knowledge and techniques to better understand this fascinating phenomenon. This knowledge is also applied in the field of biomaterials, with the aim of bringing the composition and structure, and hence the performance, of synthetic bone graft substitutes even closer to those of the extracellular matrix of bone. The purpose of this progress report is to critically review advances in mimicking the extracellular matrix of bone as a strategy for development of new materials for bone regeneration. Lab-made biomimicking or bio-inspired materials are discussed against the background of the natural extracellular matrix, starting from basic organic and inorganic components, and progressing into the building block of bone, the mineralized collagen fibril, and finally larger, 2D and 3D constructs. Moreover, bioactivity studies on state-of-the-art biomimicking materials are discussed. By addressing these different topics, an overview is given of how far the field has advanced toward a true bone-mimicking material, and some suggestions are offered for bridging current knowledge- and technical gaps.
\end{abstract}

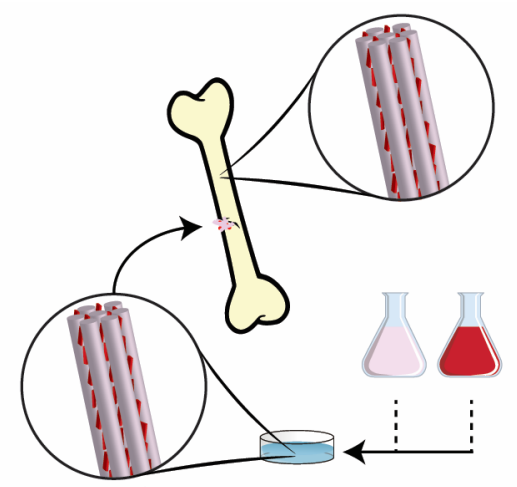




\section{Introduction}

\subsection{Bone graft substitutes}

Currently, over 2 million bone grafting procedures are performed annually worldwide, and this number is predicted to increase. ${ }^{[1]}$

Autologous bone grafting is the current standard of care for bone defects that do not heal spontaneously for a variety of reasons. The structural organization of a patient's own tissue provides an environment for osteoconduction (migration of osteogenic host cells into the transplanted material), its chemical composition and associated growth factors support osteoinduction (induction of differentiation of undifferentiated stem cells into osteoprogenitor cells), and it contains progenitor cells that are able to initiate osteogenesis (forming bone from the interior of transplanted material). In addition to that, autologous bone poses virtually no risk of immunogenic response. ${ }^{[2]}$

The drawbacks of the use of autologous bone grafts are tied with the finite supply $\left(5-70 \mathrm{~cm}^{3}\right)$ as well as with the harvesting procedure. The graft is usually taken from the iliac crest, a procedure that is associated with minor (10-39\% of cases) and major ( $0.76-25 \%$ of cases) complications. A relatively new method using a reamerirrigator-aspirator (RIA) device harvests bone fragments from the intramedullary canal with potentially lower complication rates (overall 6\%); however this method is not yet as widespread as harvesting from iliac crest bone. ${ }^{[3]}$

Eliminating the autologous harvesting procedure by using bone graft substitutes is obviously very attractive, and alternatives including allogeneic grafts (human derived), xenogeneic grafts (animal derived), or synthetic bone graft substitutes are abundantly described in the literature, and are also used in the clinical setting. ${ }^{[4,5]}$ Allogeneic and xenogeneic bone grafts suffer from disadvantages related to their origin, being the risk of immune reactions and disease transmission. Synthetic bone graft substitutes lack these drawbacks, and have the potential to perform as well as autologous bone graft, but have the additional challenge of doing so while remaining affordable.

The affordability issue precludes, to some extent, the use of cell- and growth factorbased tissue engineering scaffolds, despite some clinical successes.[6-9] Other associated drawbacks are related to cell survival upon implantation,,$^{[10]}$ and diffusion of growth factors into surrounding tissue, possibly causing ectopic bone 
formation that may lead to complications and often requires surgical intervention. ${ }^{[11-13]}$

A systematic review on biomaterials-based bone graft substitutes for use in the orthopaedics, performed in the U.K., showed that only 4 out of 59 commercially available products were demonstrated to perform equally or better than bone autograft. ${ }^{[4]}$ These products were Alpha-BSM (DePuy), ${ }^{[14,15]}$ a resorbable calcium phosphate paste, Cortoss (Orthovia), ${ }^{[16]}$ a non-resorbable bioactive glass cement, Vitoss (Orthovia), ${ }^{[17]}$ a resorbable composite of calcium phosphate and collagen, and Norian SRS (Synthes), ${ }^{[18]}$ a resorbable CaP cement. Strikingly, Vitoss is the only product showing signs of osteoinduction, a property considered essential for successful bone regeneration of large bone defects. Also important to note is that most of the products available are based on calcium phosphates, the exception being the bioglass cement, which has similar properties to CaPs in that it possesses tuneable degradability and allows direct contact with bone tissue. Despite the proven bioactivity, none of these materials are able to sustain loads by themselves, and therefore they are typically used in combination with (metallic) fixation instruments. ${ }^{[4]}$

This is the current challenge for bone graft substitutes: having load-bearing capacity comparable to cortical bone, sufficient bioactivity (osteo- conduction and induction) to elicit new bone formation, and degrading as it is replaced by newly formed native tissue. ${ }^{[19]}$ All these properties are combined in the natural bone tissue, originating from its composition and hierarchical structural organization. Natural bone is therefore a logical source of inspiration for developing novel biomaterials for bone repair and regeneration.

\subsection{Aim}

What is the state of the art in developing nature-inspired/biomimetic bone graft substitutes and what are the promises for the future?

These are the main questions that this review aims to answer, by critically analyzing recent developments in the biomaterials field, inspired from advances in the field of biomineralization, leading to materials that are close to natural bone in structure and composition. The goal is to comprehensively cover the strategies to reproduce various properties of extracellular bone matrix, ranging from the chemical composition of the mineral phase to mechanical properties stemming 
from 3D hierarchical structures. The key challenges that need to be tackled at each length scale are highlighted, as well as possible ways to overcome them.

To provide adequate background, the next section contains a short introduction to the extracellular matrix (ECM) of bone as context for discussion of achievements in bone-mimicking biomaterials research and development.

\subsection{Bone structure and composition}

Regarding the composition and structure of bone, it is important to recall that the focus is on secondary, or lamellar bone, in contrast to primary or woven bone. Secondary bone is found in mature bones, in the form of cortical (dense, compact) or cancellous (spongy, trabecular) bone. The lamellar bone tissue is remodeled from primary bone, which can be formed by endochondral ossification (mineralization of preexisting cartilage template) or by intramembranous ossification (direct bone formation).

The overall composition of the extracellular matrix of bone, excluding the various cell types that populate the matrix, is about $25 \mathrm{wt} \%$ organic component, $65 \mathrm{wt} \%$ inorganic component, and $10 \mathrm{wt} \%$ water content. Water is found in pores and channels within the bony matrix ( $5-20 \%$ of the total water present in bone), as well as in close association with the collagen fibrils and apatite mineral. In particular the latter, bound water, contributes to the plastic behavior of bone and its ability to absorb energy, with a decrease in water content due to aging resulting in a lower resistance to fracture.[20] Starting from basic components to a whole bone, an overview of structure and organization of the matrix is given below.

\subsubsection{Organic phase}

The organic matrix of bone comprises around $90 \mathrm{wt} \%$ type I fibrillar collagen and $10 \mathrm{wt} \%$ noncollagenous proteins (NCPs). Collagen molecules aggregate in triple helices - tropocollagen - that are $300 \mathrm{~nm}$ long and $1.5 \mathrm{~nm}$ wide. These are stacked longitudinally with a gap of $40 \mathrm{~nm}$, and having a lateral spacing of 1-2 nm.[21] A collagen fibril (about $100 \mathrm{~nm}$ in diameter) is made up of staggered rows of axially packed, tropocollagen molecules with lateral crosslinks (Figure 1).[22] These crosslinks can be enzymatic or non-enzymatic. The enzymatic links are further divided into immature (divalent) and mature (trivalent) crosslinks, and their total amount and ratio are related to the mechanical properties of bone, such as stiffness, 
tensile strength and fracture toughness. The non-ezymatic crosslinks result from accumulation of advanced glycation end (AGE) products and are correlated with increased bone brittleness in aging or disease. ${ }^{[23]}$ The staggering of rows creates periodic density variations in the fibril, visible in transmission electron microscopy (TEM) due to the misalignment of the gaps between molecules. ${ }^{[24]}$ These nanoscale spaces within the collagen fibril are templates that are populated by mineral during bone formation.

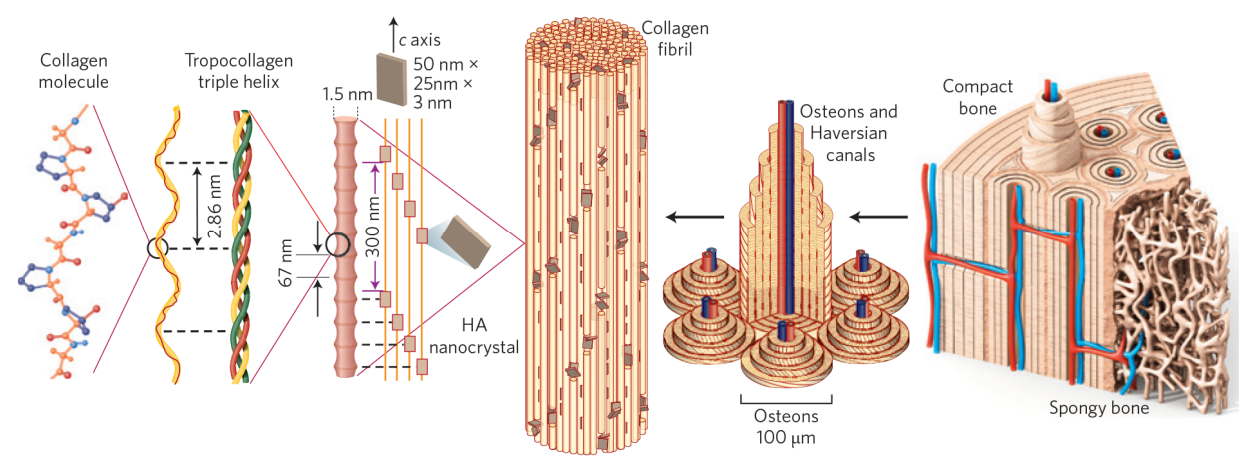

Figure 1. Schematic of the hierarchical structure of bone. Collagen molecules, with unique amino acid sequence, are assembled into triple helices, forming what is known as a tropocollagen molecule. Tropocollagen molecules come together with apatite crystallites in the collagen fibril, the building block of bone. Collagen fibrils further associates into bundles, lamellae, and finally osteons, the microscale structure of bone. Trabecular or spongy bone is porous and resides in the interior of, for example, long bones, while the compact bone is much less porous and is present at the exterior. Reproduced with permission. ${ }^{[148]}$ Copyright 2015, Nature Publishing Group.

While collagen is the main organic component of the extracellular matrix, the matrix contains a multitude of other NCPs. The roles of these proteins are still not completely understood; for a comprehensive overview of the available knowledge of these proteins we refer to the review by Zhu et al. ${ }^{[25]}$ Table 1 highlights the NCPs that are thought to have a structural role (i.e., they are present in the matrix at some point) in the formation and regulation of the extracellular matrix. This excludes enzymes (e.g., alkaline phosphatase despite its role in phosphate liberation) or growth factors (e.g., bone morphogenetic proteins despite their role 
in promoting mineralization). Most NCPs have a regulatory function in the process of biomineralization, but some also have direct implications for the mechanical properties of bone. Osteopontin (OPN) is hypothesized to play a role in preventing crack propagation: OPN-deficient mice showed 30\% less resistance to fracture.[26] OPN and osteocalcin were furthermore shown to influence the formation of submicrometer gaps, called dilational bands, between adjacent mineral aggregates. The formation of these dilational bands was correlated with diffuse damage at the micrometer scale and increased resistance to fracture. ${ }^{[27]}$

\subsubsection{Mineral phase}

Bone mineral, or bone apatite, can be found inside the collagen fibril (intrafibrillar mineral) as well as surrounding it (extrafibrillar mineral) although their relative fractions are a matter of dispute. ${ }^{[28,29]}$ In a recently published analysis of the morphology of the mineral particles, obtained by 3D reconstruction of tomography data from ion-milled bone slices, imaged by scanning transmission electron microscopy, classification in four hierarchical categories was made. Thin $(5 \mathrm{~nm}$ base), elongated (50-100 nm) and curved crystals aggregate laterally (width 20-30 $\mathrm{nm}$ ) to form the platelets often described in literature. ${ }^{[30]}$ These can be further organized into thicker stacks of two to four platelets, or into aggregates of all the previous structures, forming complex shapes up to $300 \mathrm{~nm} \cdot{ }^{[31]}$

The chemical composition of bone apatite is far from the stoichiometric hydroxyapatite, as it is highly substituted with ions from the physiological fluids. These substitutions can occur in any of its four crystallographic domains: the $\mathrm{PO}_{4}{ }^{3-}$ tetrahedral positions, the $\mathrm{Ca}^{2+}(\mathrm{I})$ positions (phosphate surrounded hexagonal columns) or $\mathrm{Ca}^{2+}(\mathrm{II})$ positions (staggered triangular surrounding hydroxide) or in the $\mathrm{OH}^{-}$"channel" positions. ${ }^{[32]}$ The precise composition of ion substitutes tends to vary among subjects and depending on the type of bone itself, but in general, the following ions are present: $\mathrm{CO}_{3}{ }^{2-}$ (around $5 \mathrm{wt} \%$ ), $\mathrm{Na}^{+}$and $\mathrm{Mg}^{2+}$ (both 0.5-1 $\mathrm{wt}^{\%} \%$ ), ${ }^{[30,33,34]} \mathrm{K}^{+}, \mathrm{Sr}^{2+}, \mathrm{Cl}, \mathrm{F}^{-}$(up to $0.1 \%$ ), and trace amounts (in the ppm range) of several other metallic elements: $\mathrm{B}, \mathrm{Al}, \mathrm{Si}, \mathrm{Ti}, \mathrm{Cr}, \mathrm{Mn}, \mathrm{Fe}, \mathrm{Co}, \mathrm{Zn}, \mathrm{Sr}, \mathrm{Cd}, \mathrm{La}$, and $\mathrm{Pb}$. ${ }^{[35-37]}$ There is debate about whether the crystallographic unit cell is hexagonal or monoclinic. ${ }^{[30,38]}$

Mineral platelets are hypothesized to be closely related to the citrate anion, which is present in high concentration in bone. ${ }^{[39]}$ The role of citrate is not yet well defined 
although evidence exists relating it to the restriction of apatite crystal growth[40,41] and to the stabilization of an amorphous calcium phosphate phase. ${ }^{[2]}$

Table 1. Noncollagenous structural proteins involved in the formation or regulation of the extracellular matrix

\begin{tabular}{ll}
\hline Non-collagenous protein & Known or hypothesized role \\
\hline $\begin{array}{l}\text { Large proteoglycans } \\
\text { Versican (VCAN) }\end{array}$ & $\begin{array}{l}\text { Present in early stages of bone development. }{ }^{[149]} \text { Contains } \\
\text { EGF-like sequences that may affect osteogenic } \\
\text { differentiation. }{ }^{[150,151]}\end{array}$ \\
$\begin{array}{ll}\text { Small leucine-rich repeat proteoglycans (SLRPs) } \\
\text { Decorin (DCN) } \\
\text { and biglycan (BGN) }\end{array}$ & $\begin{array}{l}\text { Regulators of collagen fibrillogenesis. }{ }^{[152,153]} \text { Decorin possibly } \\
\text { modulates collagen mineralization. }{ }^{[154]} \text { Biglycan is involved in } \\
\text { angiogenesis during fracture healing, }{ }^{[155,156]} \text { and osteoblast } \\
\text { differentiation. }{ }^{[157]}\end{array}$ \\
Osteomodulin (OMD) & $\begin{array}{l}\text { Involved in the organization of the collagen matrix. } .^{[158]} \\
\text { Asporin (ASPN) }\end{array}$ \\
Fibromodulin (FMOD) & Possible role in collagen mineralization. ${ }^{[159,160]}$ \\
and Lumican (LUM) &
\end{tabular}

\section{Glycoproteins}

Osteonectin (SPARC) Affinity to collagen and hydroxyapatite, involved in collagen mineralization. ${ }^{[162,163]}$

Tetranectin (CLEC3B)

Possibly involved in fracture healing. ${ }^{[164]}$

RGD-containing Glycoproteins

Thrombospondin-2 (THBS2)

Involved in the organization of the collagen matrix. [165]

Fibronectin (FN1)

Possibly providing attachment points to various cell types.[166]

and Vitronectin (VTN)

Small integrin-binding ligans with N-linked Glycosilation (SIBLINGs)

Osteopontin (SPP1)

Bone sialoprotein (IBSP)

Affinity to collagen and hydroxyapatite, known inhibitor of ectopic calcification, modulator of bone mineralization.[167]

Affinity to collagen and hydroxyapatite, induces

differentiation of osteoblasts and is a mineralization promoter. ${ }^{[168,169]}$

Dentin Matrix Protein 1 (DMP1)

Involved in bone mineralization, likely promoter of mineralization. ${ }^{[51,170]}$

Matrix extracellular

phosphoglycoprotein (MEPE)

Involved in bone mineralization, likely inhibitor of

Bone acidic glycoprotein 75 mineralization. [171-173]

Possibly involved in early stages of bone mineralization. ${ }^{[174-176]}$

(BAG-75) 
$\gamma$-Carboxy glutamic acid proteins

Matrix-Gla-protein (MGP)

Osteocalcin (BGLAP)

Serum proteins

Albumin (ALB) and

$\alpha_{2}$-HS-glycoprotein (AHSG)
Inhibitor of mineralization. ${ }^{[177,178]}$

Regulator of mineralization. ${ }^{[173,179,180]}$

Both have affinity to $\mathrm{Ca}^{2+}$, inhibiting hydroxyapatite mineralization by arresting crystal growth. AHSG does so to a greater extent than albumin. ${ }^{[181,182]}$

\subsubsection{Mineralized collagen fibril}

The components described so far, the structural collagen matrix, noncollagenous proteins and molecules, and the apatite platelets, come together in what is considered to be the building block of the extracellular matrix of bone: the mineralized collagen fibril (Figure 1).

The small dimensions of crystallites, their highly substituted chemical phase, and close associations with collagen and other organic molecules make it rather difficult to precisely characterize this unit block although more is known about its composition (described in the previous section) than about the mechanisms by which it is formed. The 3D arrangement of apatite and collagen at the nanoscale has also been recently described. ${ }^{[31]}$

The mounting evidence for amorphous precursors in the formation stages of bone from different vertebrates, namely in zebrafish fin rays, ${ }^{[43-45]}$ mouse calvaria and long bones, ${ }^{[46,47]}$ as well as in enamel in mouse, ${ }^{[48]}$ points toward a non-classical mineralization pathway. ${ }^{[49]}$ This is substantiated with an in vitro study on the formation of amorphous calcium phosphate $(\mathrm{ACP})$ that correlates with the $\mathrm{ACP}$ phase found in vivo. ${ }^{[50]}$ The process of collagen mineralization by an amorphous precursor is likely assisted by NCPs that stabilize ACP and interact with collagen, as shown in in vitro models for DPP, DMP1, ${ }^{[51,52]} \mathrm{OPN},{ }^{[53]}$ and AHSG, ${ }^{[54-56]}$ as well as by a study on remineralization of DBM. ${ }^{[57]}$ Carbonate has also been hypothesized to have a role in the formation of first mineral domains, but at the moment only limited in vitro evidence exists.[58-60] Therefore, much is still to be discovered about the mechanisms of mineralization regulated by organic components.[61] 


\subsubsection{Fibril arrays, lamella and osteons}

Mineralized collagen fibrils (about $100 \mathrm{~nm}$ ) bundle together in fibril arrays (sometimes called fibers), and these are arranged in parallel stacks. A lamella consists of typically parallel fibril arrays (in trange of $100 \mathrm{~nm}$ to $10 \mu \mathrm{m}$ ). Lamella can be stacked in different ways, ${ }^{[62]}$ for example, in parallel stacks, with a slight angle offset, or alternating at sharp angles. ${ }^{[63,64]}$

Osteons are the hallmark structure of secondary bone, and they have a diameter of around $100 \mu \mathrm{m}$. An osteon consists of a central (Haversian) canal, occupied by a blood vessel, and surrounded by concentric lamella (Figure 1). Canals perpendicular to the Haversian canal, the so-called Volkmann canals, link adjacent osteons. Lacunae, with resident osteocytes, populate the bone surrounding the Haversian canal, with their long axis concentric to the Haversian canal. Canaliculi extend from the lacunae, linking adjacent osteocytes to each other and to a filtering surface.

\subsubsection{Cortical and trabecular bone}

Cortical bone forms the hard shell-like surface of a bone, while trabecular bone fills its core. Porosity distinguishes cortical $(\approx 6 \%)$ and trabecular bone $(\approx 80 \%))$. While osteons are packed together in cortical bone, in trabecular bone the mineralized matrix is sparse, consisting of interconnected struts of about $200 \mu \mathrm{m}$ (Figure 1). These trabeculae undergo constant remodelling, and their orientation and local density depend on the typical load applied to the bone.[21] The pores in this spongelike structure house the bone marrow.

\section{Biomineralization-inspired materials}

In the following section, examples of biomaterials are given that possess closer-tobone characteristics. The focus is on discussing the state-of-the-art of bonemimicking or bone-inspired biomaterials, and having a critical comparison of such materials with the natural extracellular matrix.

\subsection{Mimicking the building block of bone}

When ascertaining to what level an artificially produced mineralized collagen fibril replicates the building block found in natural bone, there are several key points to 
keep in mind (Figure 2). One is the high mineral content of the natural nanocomposite, of around $65 \mathrm{wt} \%$, that can, for example, be shown by thermal gravimetric analysis (TGA) (Figure 2g). The second is a homogenous mineralization throughout the developed substrate that can be demonstrated using elemental surface analysis methods such as energy dispersive X-ray spectroscopy (EDS) mapping performed on different areas (Figure $2 \mathrm{~b}-\mathrm{c}$ ). Third, there must be evidence of the close relationship between the apatite mineral and the collagen matrix, which can be observed by scanning electron microscopy (SEM) and EDS, or TEM. Fourth, proof that mineralization is intrafibrillar, which can be obtained by a combination of indirect methods, or directly, by the use of 3D imaging techniques. Indirectly, intrafibrillar mineralization can be evidenced by a combination of techniques that show that the composite has a high mineral content (e.g., TGA), while the normal ultrastructure and surface of the organic matrix is maintained (e.g., SEM-EDS), leading to the conclusion that the mineral must reside within the organic matrix. The latter analysis should show that at early time points, no extrafibrillar mineral is present, as mineralization typically starts from the inside out, leaving the collagen surface unaltered (Figure 2a). Unequivocal proof of intrafibrillar mineralization can be obtained with 3D imaging techniques such as cryogenic electron tomography (Figure $2 \mathrm{~h}$ ) or, alternatively, by clever use of superresolution microscopy techniques such as stochastic optical reconstruction microscopy, used to image fluorescent calcein-labeled apatite, to show its localization within immuno-labeled collagen fibrils (Figure 2i). Last, it is important to show that the mineral present is similar to bone apatite, in composition, particle size and orientation. Data from selected- area electron diffraction (SAED) in combination with TEM imaging covers all three, by revealing the angles and dspacing characteristic of apatite mineral and proving that the orientation of the crystallographic c-axis is along the longitudinal axis of the collagen fibril (Figure 2d-f). 

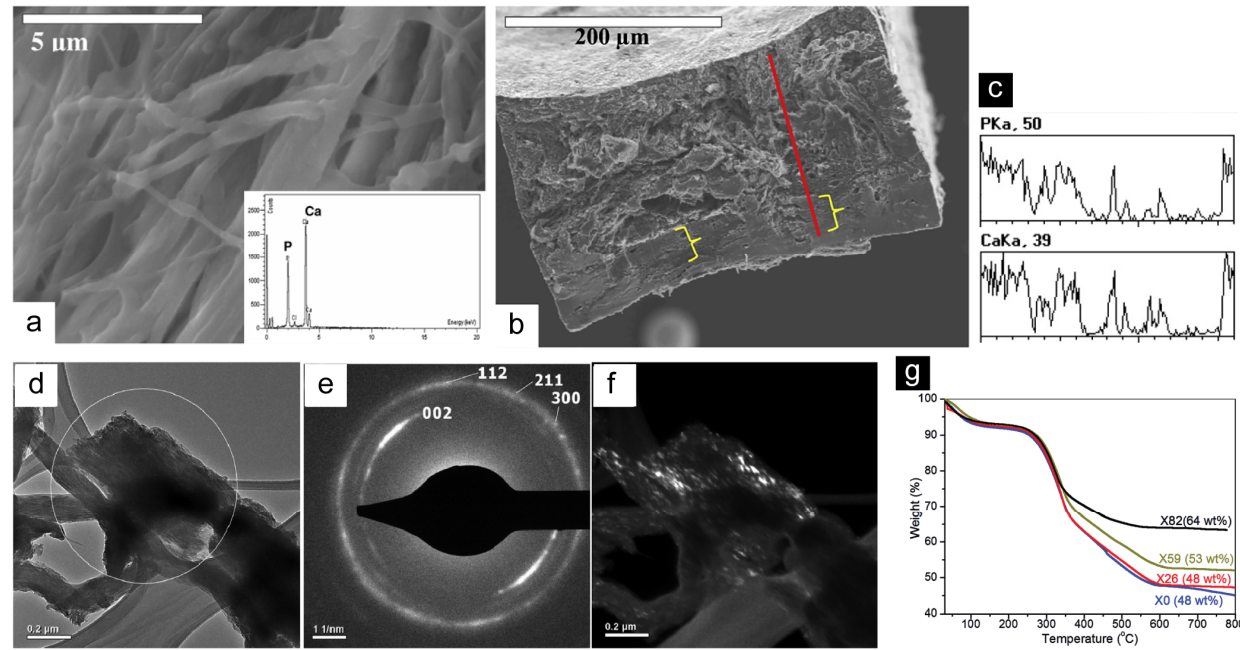

h
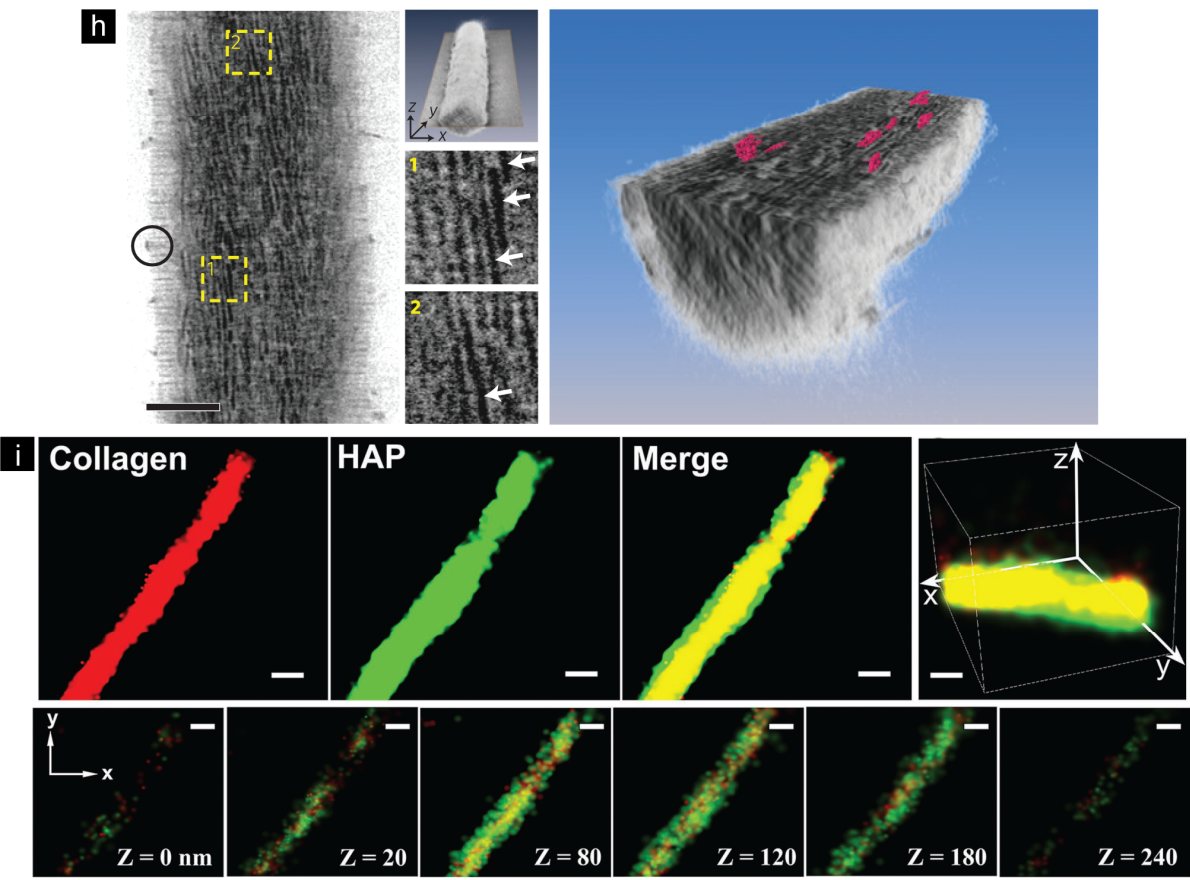

Figure 2. Characterization of intrafibrillar-mineralized collagen. (a) SEM image of a collagen scaffold mineralized for 3 days, showing a typical surface morphology of the organic matrix, while EDS spectrum (inset) shows presence of $\mathrm{Ca}$ and $\mathrm{P}$, associated with $\mathrm{CaP}$ mineralization; (b) SEM image of a remineralized piece of manatee bone, with (c) EDS spectra collected along the red line, showing heterogeneous $\mathrm{Ca}$ and $\mathrm{P}$ distribution on the cross section, suggesting that infiltration of the pAsp-ACP complex was not complete; (d) 
bright field TEM image of a mineralized collagen fibril and (e) corresponding SAED pattern, showing characteristic 002 reflection aligned with the fibril, and ring composed of the 112, 211, and 300 reflections; (f) dark field TEM image made with the beam from the 002 reflection, showing crystals distribution within the collagen fibril; (g) TGA of PILPmineralized collagen sponges showing that increasing degree of crosslinking ( $\mathrm{X} 0$ : control, $\mathrm{X} 26<\mathrm{X} 59<\mathrm{X} 82)$ promotes increase in mineral content; (h) cryo-ET of a mineralized collagen fibril showing the plane of section through the middle of the fibril, with magnified regions identified by yellow squares, where apatite platelets are visible as dark streaks (white arrows). On the right a 3D reconstruction of the apatite platelets embedded in the collagen fibril: (i) STORM images of calcein-labeled apatite (red) and immunostained collagen (green), imaged in a z-stack ranging 0-240 nm, showing the presence of apatite within the collagen fibril. (a) Reproduced with permission.[77] Copyright 2010, Elsevier. (b), (c) Reproduced with permission. ${ }^{[69]}$ Copyright 2011, Elsevier. (d), (e), (f), (g) Reproduced with permission. ${ }^{[80]}$ Copyright 2011, American Chemical Society. (h) Reproduced with permission. ${ }^{[56]}$ Copyright 2010, Nature Publishing Group. (i) Reproduced with permission. ${ }^{[97]}$ Copyright 2018, Wiley-VCH.

In the context of reconstituting the main building block of bone, i.e., the mineralized collagen fiber, the discovery of the so-called polymer-induced liquid precursor (PILP) process has had a major impact. This process, which was observed in the in vitro experiments on the stabilization of amorphous mineral phases, was initially described for calcium carbonate ${ }^{[65]}$ and later for CaP. ${ }^{[30]}$ The PILP process encompasses, in the case of $\mathrm{CaP}$, the stabilization of hydrated clusters of $\mathrm{Ca}_{2}\left(\mathrm{HPO}_{4}\right)_{3}{ }^{2-}$ that form from saturated solutions, ${ }^{[50]}$ by charged (typically anionic) polymers, effectively creating stable amorphous calcium phosphate-polymer particles.

The ACP-polymer complexes are capable of infiltrating pre-assembled collagen fibrils through the a-band of the $40 \mathrm{~nm}$ gap region of collagen. ${ }^{[56]}$ The mineral then crystallizes inside the fibril, resulting in mineralized collagen fibrils that have a remarkable resemblance to mineralized collagen found in natural bone. More specifically, the intrafibrillar mineral consists of crystallites with the c-axis orientation in the direction of the fibril.[30] The exact mechanism of infiltration is still under debate, and various hypotheses suggest the importance of electrostatic interactions, ${ }^{[50,56]}$ capillary-like forces, ${ }^{[30]}$ mineralization by inhibitor exclusion, ${ }^{[55]}$ or simultaneous fibrillogenesis and apatite formation.[66] Interestingly, the fact that intrafibrillar mineralization of collagen was achieved via an amorphous precursor in vitro prompted the biomineralization community to renew efforts in 
investigating the role of such precursors in the in vivo biomineralization process. These efforts resulted in the confirmation that indeed, in the process of formation of zebrafish fin rays, and mouse calvaria, long bones, and enamel, an amorphous precursor is present, as mentioned in the introductory section. ${ }^{[43,45-48,67]}$ Since early 2000, a range of experiments have been performed that have increased our understanding of the process of intrafibrillar mineralization of collagen and bone biomineralization, as well as provided useful tools for developing truly biomimetic materials for bone regeneration.

A large majority of approaches to obtain intrafibrillar collagen mineralization employs polymeric process-directing agents; however, in few studies the process was performed in the absence of NCPs or their analogs. For example in a study by Wang et al., the authors employed simultaneous collagen fibrillogenesis and precipitation of carbonated apatite. This approach proved capable of forming intrafibrillar mineral, with orientation of the crystallographic c-axis in the collagen fibril direction, as shown by TEM-SAED and SEM microscopic images. The mineral content, determined using TGA, was $32 \mathrm{wt} \%$ after a 4 day reaction (calculated relative to the dry composite, taken as $\mathrm{wt} \%$ left at $200^{\circ} \mathrm{C}$ ). The mineralization was extended by a 16 day immersion in a simulated body fluid, resulting in an increase of mineral content up to $50 \mathrm{wt} \%$ of the dry mass. ${ }^{[66]}$ While this work proved that NCPs, or their analogues are not necessary to achieve intrafibrillar mineralization of collagen, the mineralization extent in this study falls somewhat short of the expected $65 \mathrm{wt} \%$. Furthermore, while it is expected that the mineral deposited during the first phase is homogeneously spread throughout the scaffold, this is less certain for the second phase, where the substrate is already dense and mineralized before immersion in the SBF${ }^{1}$ solution. Indeed, cryo-TEM micrographs of the scaffold showed fibrils ranging from not mineralized to fully mineralized. Complementary SEM-EDS analyses at different depths across the 3 $\mathrm{mm}$ thick disc would have given a valuable insight in the homogeneity of mineral distribution throughout the dense substrate.

In another study by Marelli et al., it was hypothesized that the $\mathrm{pH}$ at which collagen fibrils are formed, influences intrafibrillar mineralization. Using cationic or anionic dyes, it was confirmed that collagen gels formed under mild alkaline conditions $(\mathrm{pH}=9.0)$ had more negative charges than gels formed under neutral

\footnotetext{
${ }^{1}$ Simulated body fluid
} 
conditions $(\mathrm{pH}=7.4)$. After mineralization in SBF for 14 days, the negatively charged gel had $64 \mathrm{wt} \%$ more mineral deposited than the positively charged one. Although this was presented as intrafibrillar mineral, no conclusive evidence was provided to support this statement. The TEM image of the gel after $24 \mathrm{~h}$ of incubation in SBF showed mineral agglomerates together with a collagen fibril, but the exact location of the mineral (adjacent to, on top of, or inside the fibril) was not clear. The supplementary SEM images strongly suggested that the mineral formed was actually extrafibrillar, and the authors were careful to phrase the figure captions as "mineral formation within the collagen framework," which suggests that the formation occurred inside the collagen gel, but that it was not necessarily intrafibrillar.

Among the different polymeric additives that have been explored in the context of mineralization with process-directing agents, poly(aspartic acid) (pAsp) is the most commonly used. ${ }^{30,52,56,57,68-80]}$ Other polymer additives include poly(glutamic acid) (pGlu), ${ }^{[79,81,82]}$ poly(amidoamine) dendrimers, ${ }^{[83,84]}$ poly(acrylic acid) (PAA), ${ }^{[69,81]}$ typically used in combination with poly(vinylphosphonic acid) (PVPA), $[79,81,85,86]$ sodium trimethaphosphate (STMP), ${ }^{[87]}$ poly(glutamic acid), ${ }^{[88]}$ or phosphorylated collagen. ${ }^{[89,90]}$ The only example of a cationic polymer able to induce intrafibrillar mineralization of collagen is the one of poly(allylamine) hydrochloride (PAH). ${ }^{61,91-}$ 93]

It is hypothesized that the high content of anionic side chains in, e.g., pAsp, as well as its nonglobular, flexible nature, mimic the composition and structure of certain acidic NCPs found in vivo such as OPN, DMP1 and DPP, known to be involved in biomineralization processes. ${ }^{[94,95]}$ These are classified as intrinsically disordered proteins that have a high content of charged side chains and lack a well-defined tertiary structure. However, not all aspects of the NCPs are present in polymeric analogs: often they lack the integrin- or collagen-binding sequences, phosphorylated serine/threonine content, or post-translation modifications such as glycosylation, and the role of these NCP motifs in biomineralization remains elusive. ${ }^{[96]}$ The exception is perhaps the role of phosphorylated side chains, which were mimicked by the use of PVPA as process-directing agent, or by pre-treating collagen with STMP. PVPA, either in solution or crosslinked to collagen, was able to induce intrafibrillar mineralization of reconstituted collagen fibrils and demineralized dentin, but only in the presence of PAA. ${ }^{[81,86]}$ The phosphorylation of 
collagen fibrils by SMTP before immersion in a mineralization solution (stabilized by PAA) was also shown to produce intrafibrillar mineral in reconstituted fibrils and demineralized dentin. ${ }^{[85,87]}$

These studies seem to suggest that the use of phosphorylated groups has a role in guiding the formation of intrafibrillar mineral, but not in stabilizing ACP itself, as this was accomplished by another biomimetic analog (PAA). The lack of quantification of mineral content in the collagenous matrix, for example by TGA, makes comparison with other mineralization strategies difficult. Moreover, the rather complicated mineralization setup used in these studies, consisting of a combination of set Portland cement (as $\mathrm{Ca}^{2+}$ and $\mathrm{OH}^{-}$source) and a modified SBF (as $\mathrm{PO}_{4}{ }^{3-}$ source), as well as the necessary use of two biomimetic analogs make this strategy rather complex for the results it yields.

Comparative studies on the effect of polymer concentration ${ }^{[79]}$ and molecular weight ${ }^{[77]}$ point toward pAsp of high molecular weight (highest reported is $32 \mathrm{kDa}$, but $27 \mathrm{kDa}$ is more commonly used) as the best process-directing agent for achieving mineral content between 60 and $70 \mathrm{wt} \%$, comparable to that of native bone. Similar ranges of mineral content were obtained by combinations of pGlu with pAsp, but there was no added benefit from the incorporation of pGlu.

The influence of polymer concentration is harder to gauge from the existing literature, because for many studies, the reaction volume is not stated, and similar mineral content $(\mathrm{wt} \%)$ is achieved with different concentrations, ranging from 10 to $100 \mu \mathrm{g} / \mathrm{mL}$. One study analyzed the effect of concentration of pAsp (molecular weight $2-11 \mathrm{kDa}$ ) below $10 \mu \mathrm{g} / \mathrm{mL}$, finding that the rate of infiltration and mineralization slowed down with increasing concentration of the process-directing agent. ${ }^{[52]}$ However, these results were obtained for collagen fibrils reconstituted on TEM grids, and the mineralization solution was less concentrated than typical PILP preparations ( $2.7 \mathrm{mM} \mathrm{CaCl}_{2}$ and $1.35 \mathrm{mM} \mathrm{K}_{2} \mathrm{PO}_{4}$ ), making the comparison with other studies that often use bulk reactions for millimeter-sized substrates, difficult. Another aspect that has been shown to affect the mineral content upon PILPdriven mineralization is the extent of collagen crosslinking. For example, it has been demonstrated that a collagen sponge exhibited increased mineral content (48, 53 , and $64 \mathrm{wt} \%)$ with an increase in crosslink density $(26 \%, 59 \%$, and $82 \%$ of free amine groups in collagen). ${ }^{[80]}$ The mineralization reaction of a $10 \mathrm{mg}$ reconstituted collagen sponge using $10.5 \mathrm{kDa}$ pAsp $(50 \mu \mathrm{g} / \mathrm{mL}$ in $100 \mathrm{~mL})$ lasted for 14 days. 
When a (non-crosslinked) commercial sponge was used, the same level of mineralization was achieved after 16 days; however, in the latter case, the reaction volume used was 5 times higher (10.3 kDa pAsp $(50 \mu \mathrm{g} / \mathrm{mL}$ in $500 \mathrm{~mL})$ ). [77] Taken together, these studies suggest that the crosslinking of collagen substrates is an efficient way of improving mineralization kinetics. Moreover, crosslinking density was correlated with increasing stiffness and hardness of the composite, with the highest crosslink density (82\%) showing elastic modulus comparable to that of woven bone (around $0.2 \mathrm{GPa}$ ), even in the wet state. ${ }^{[80]}$

In a recent study by Shao et al., citrate was also shown to increase the mineral content of reconstituted collagen and demineralized dentin, when used in combination with pAsp-assisted mineralization. Collagen gels were pretreated in a citrate-containing solution, before immersing in mineralization medium, and showed increased residual mineral $\mathrm{wt} \%$ with increasing concentration of citrate. ${ }^{97]}$ One aspect that has not been extensively discussed or investigated is the influence of the nature or properties of the collagen substrate itself on the mineralization by the PILP process. It is known that the nanometer-sized compartments, created during fibrillogenesis, and discernible through the emergent $67 \mathrm{~nm}$ periodic Dspacing, are necessary for infiltration of the hydrated complexes, and also have a role in directing crystallization and determining the final morphology of the apatite mineral. ${ }^{[56]}$ Proper fibril formation appears to be the only necessary condition for subsequent intrafibrillar mineralization. However, the collagen scaffolds used in the studies reported here have many different origins and have undergone different processing. For example, collagen substrates have been used as reconstituted fibrils or sponges (sometimes called membranes) from acid- or enzyme-extracted collagen (that can be prepared in-house or purchased). They can originate from rat-tail tendon, ${ }^{[61,75,91]}$ bovine ${ }^{[77,79,90,92,93]}$ or equine tendon, ${ }^{[52,56]}$ or bovine skin. ${ }^{30,68,80,81,83,85]}$ Substrates have also been prepared directly from biological tissue, such as turkey tendon, ${ }^{[78]}$ demineralized sections from mouse periodontium, ${ }^{[57]}$ and bone from manatee ${ }^{[69,79]}$ or fish ${ }^{[82]}$ origin, as well as demineralized dentin from mouse, ${ }^{[72,73]}$ or human origin. ${ }^{[70,71,74,86-89]}$

The versatility of the PILP process is attested by the mineralization results with all these substrates. But considering the differences between the reactions employed conditions of fibril formation, molecular weight and concentration of the additive, reaction volume and time - one may question whether there is an underlying 
relation between the conditions required for successful mineralization and the properties of the substrate. In other words, are we simply selecting the conditions that work for a certain substrate, but missing some key aspect in the overall mechanism? This is supported by sporadic observations of heterogeneity of mineral deposition, more so in reconstituted than in tissue-derived collagen; and also by (possibly underreported) failed mineralization reactions upon aging of a collagen substrate known to have worked previously.96] With the available information, this conclusion remains speculative and therefore, more systematic studies are needed on the influence of collagen origin and processing on the efficiency and kinetics of mineralization.

In addition to pAsp and other polymers described above, whole (recombinant or purified) proteins have also been used for collagen mineralization studies, as mentioned in Section 1. ${ }^{[51,53-56]}$ Inspired by the native proteins, some tailor-made peptides and proteins have been developed and shown to play a role in $\mathrm{CaP}$ precipitation in vitro, ${ }^{[98]}$ although a few attempts have been made to use them as NCP analogs in collagen mineralization studies. Sfeir et al. used a peptide containing a DPP-like motif, giving some insight into the possible phosphorylation mechanism of sequential serine residues, often found in NCPs. ${ }^{[99]}$ Ping et al. used a multi-functional protein, containing a BSP-sequence and a HAP-binding motif that was shown to promote intrafibrillar mineralization of collagen in the presence of poly(acrylic acid).[100] These studies have made an important contribution to the existing knowledge regarding the mechanisms of bone mineralization. Nevertheless, the number of studies in which tailor-made NCP analogs like these have been used to develop in vitro building blocks of bone is much lower than the studies where the far simpler synthetic polymers described above were used. Furthermore, the extent of mineralization obtained using these methods seemed to be inferior toi.e., pAsp-driven mineralization.

Taken together, it can be concluded that our knowledge of the roles and importance of NCPs is still relatively limited. Nevertheless, continued research on biomineralization-related synthetic peptides and proteins is warranted as such compounds may be useful in controlling, for example, the extrafibrillar mineral formation, in enhancing mechanical properties of synthetic bone substitutes, but 
also in less obvious ways, as replacements of collagen as the structural matrix, as described in Section 2.3 .

\subsection{Mimicking the biological apatite}

Where the previous section focused on the methods to replicate the intimate contact between apatite and collagen, here we zoom in into the properties of the mineral phase formed during intrafibrillar mineralization of collagen. It should be noted that while a large body of literature exists on methods to produce bone-like apatite (e.g., described in several reviews ${ }^{[101,102]}$ ), here only the studies concerning mineral formed during intrafibrillar mineralization of collagen are discussed. In the previous section, it was shown that some excellent results were obtained on the nanoscale organization of organic and inorganic constituents of bone-like building blocks by using intrafibrillar mineralization of collagen. In contrast, less is known about the chemical composition of the deposited mineral phase, specifically regarding ionic substitutions. Characterization of intrafibrillar mineral is mostly limited to TEM-SAED and X-ray diffraction (XRD) data, showing crystallite size and orientation, and crystallographic reflections matching those of bone apatite (TEM-SAED), ${ }^{[30]}$ corroborated by similar XRD patterns between synthetic scaffolds and compact bone. ${ }^{[80]}$

Small amounts of ions can sometimes have large effects of normal functioning of organs and tissues and on their repair and regeneration. ${ }^{[103,104]}$ This is the concept behind the application of bioinorganics, which involves the use of (trace amounts of) ions to stimulate the regeneration of damaged tissue, much like a (organic) growth factor would do. This concept as well as various methods of incorporation of bioinorganics into, e.g., bone graft substitutes has been extensively discussed in several reviews. ${ }^{[102]}$ It is therefore interesting to investigate whether bioinorganics can also be incorporated into the mineral deposited during intrafibrillar mineralization of collagen. This would not only make "closer-to-nature" materials, in terms of their chemical composition, but would also plausibly enhance their bone regenerative potential. Only a few examples exist of such studies, although some substitutions are to be expected due to the composition of the mineralization solution employed, containing carbonate, sodium, and potassium ions. 
Characterization of the carbonate moiety by FTIR is typically done by deconvolution of the $v_{2} \mathrm{CO}_{3}^{2-}$ band between 850 and $890 \mathrm{~cm}^{-1}$ into three different peaks: at $866 \mathrm{~cm}^{-1}$ for unstable location, $871 \mathrm{~cm}^{-1}$ for B-substitution ( $\mathrm{PO}_{4}^{3-}$ position) and $878 \mathrm{~cm}^{-1}$ for A-substitution ( $\mathrm{OH}^{-}$position). ${ }^{[105]}$ Inductively coupled plasma optical emission spectroscopy (ICP-OES) or mass spectrometry (ICP-MS) can provide good quantification of $\mathrm{wt} \%$ of metallic ions, although by itself it is not sufficient to determine the exact position of the dopant, which can be incorporated into the crystalline lattice, adsorbed on the surface, or associated with the surrounding organic molecules. A complementary analysis by Rietveld refinement of diffraction data can substantiate evidence for the location of a substitute ion. This approach was used to characterize substitutions by $\mathrm{Mn}^{2+}, \mathrm{Co}^{2+}$ and $\mathrm{Ni}^{2+}$ in nanometer-sized citrate-carbonate-apatite (albeit in the absence of collagen, which could admittedly complicate the analysis), with good agreement between the theoretical pattern (calculated for a certain \% of substitution in $\mathrm{Ca}^{2+}$ - data obtained by ICP-OES) and the experimental data.[106] Refinement of the diffraction data was also used for characterizing $\mathrm{Mg}^{2+}$-substituted carbonated apatite, and identifying preferential substitution in the $\mathrm{Ca}(\mathrm{II})$ position.[107] Other characterization methods to differentiate between adsorbed or lattice-incorporated dopants include ${ }^{13} \mathrm{P},{ }^{1} \mathrm{H}$, or ${ }^{43} \mathrm{Ca}$ solid-state nuclear magnetic resonance spectroscopy (NMR), that can give information about the location of cation substitutions, ${ }^{[108-110]}$ and small- and wideangle X-ray scattering (SAXS and WAXS), which provides measurements of the lattice parameters, which are typically affected by substitutions from dopant ions with different radii. Additionally, SAXS/WAXS can also provide spatial resolution when used in the scanning mode.[111] Combination of electron microscopy with electron energy-loss spectroscopy (EELS) also offers chemical information with spatial resolution, as shown in a study where the formation of mineral deposits by mouse osteoblasts was observed to start from a $\mathrm{CO}^{2}{ }^{2-}$-rich ACP nodule.[60] Other useful methods of chemical mapping include time-of-flight secondary-ion mass spectrometry, ${ }^{[112,113]}$ and atom probe tomography. ${ }^{[114]}$

Although in general, the techniques that have been used for chemical and structural characterization of bone can also be applied to the intrafibrillar mineral produced in vitro, to the best of our knowledge, they have been used to a very limited extent in biomineralization studies. One study mentioned that the addition 
of strontium to the mineralization solution "strongly disrupted the [PILP] process", although no data were provided. ${ }^{96]}$ Strontium is known to decrease osteoclast-resorbing activity and differentiation, while promoting osteoblast differentiation, ${ }^{[115,116]}$ which makes it attractive for bone regeneration strategies. Another study focused on the interference of copper that was included in the mineralization solution by dissolution from TEM grids. The ion was shown to completely prevent mineralization by the PILP process, by over-stabilizing the ACP precursor, which was shown to infiltrate the collagen fibrils but remain amorphous for at least 72 hours.[52] Copper is a known stimulator of angiogenesis, ${ }^{[117]}$ which is of great value for large-defect regeneration.

Clearly, further research is necessary on the role of "foreign" ions in the polymerinduced mineralization process. It is important to understand whether they can be incorporated during the amorphous precursor formation phase or, as in the case of copper (and apparently strontium), they prevent the mineralization process altogether. This knowledge would not only increase our understanding of natural mineral formation, but could also aid the development of better performing bone graft substitutes.

\subsection{Beyond biomimetics: replacing collagen as the structural matrix for biomineralization}

Exploring synthetic biomaterials as a template for intrafibrillar-like mineralization can contribute to the development of novel bone graft substitutes that do not rely on collagen sources. In general, collagen used in these types of studies and developments is animal-derived, and finding synthetic alternatives paves the way toward more ethically responsible and sustainable strategies for bone regeneration. Another advantage of replacing collagen as the organic structural matrix is that by using a designed and more controlled system, it is possible to better understand the role of individual properties of both the structural template and the processdirecting agent in the biomineralization process. Replicating the nanoscale organization of the organic-inorganic components of bone with synthetic materials is, however, not trivial, because it is in part due to the collagen structure and composition that the organization of the apatite phase takes place, as pointed out by various studies. $[55,56,66]$ 
Nevertheless, a successful development was shown using the recombinant proteins elastin-like recombinamers (ELRs). These are recombinant proteins, inspired from a sequence derived from elastin, and capable of undergoing a soluble-insoluble transition at a critical temperature (termed inversed transition temperature $T_{t}$ ), aggregating in a stable conformation. For temperatures above $T_{t}$, ELRs were shown to undergo intrafibrillar-like mineralization (Figure 3). Characterization by TEM-SAED showed that the self-assembled and crosslinked ELR matrix was mineralized with randomly oriented, needle-like apatite crystals. X-ray diffraction confirmed the resemblance with bone apatite, and SEM-EDS showed maintenance of the hydrogel structural morphology and porosity despite extensive mineralization. Furthermore, mechanical characterization of the mineralized ELR was performed by nanoindentation both in dry and wet states, with bovine cortical bone used as control. The mineralized ELR had an elasticity modulus of $20.3 \mathrm{GPa}$, comparable to that of cortical bone with a modulus of elasticity being $25 \mathrm{GPa}$. The hardness of mineralized ELR matrix and cortical bone in the dry state was 0.93 and $1.2 \mathrm{GPa}$, respectively. In the wet state, the mechanical properties of mineralized ELR were about one-third of those of cortical bone.[118]

A subsequent study by the same group gave evidence that the spaces created within ELR fibrils were responsible for its infiltration with the ACP precursor, and subsequent intrafibrillar mineralization. By using pAsp or PAH as processdirecting agents that create, respectively, negative and positive charged ACPpolymer complexes, mineralization of the positively charged ELR (due to lysine residues) occurred in both cases. This suggests that electrostatic interactions are not the driving force behind the infiltration of an amorphous phase, as mentioned in other studies.[56] It was also observed that the ELRs containing bioactive sequences formed gels that were less stable than the gels formed by ELRs containing the backbone sequence alone. It was hypothesized that the bioactive sequences disrupted the otherwise stable secondary structures $-\beta$-spirals - that form at temperatures higher than $T_{\text {t. }}$. However, mineral was reported to be found within the fibrils of all ELRs. 

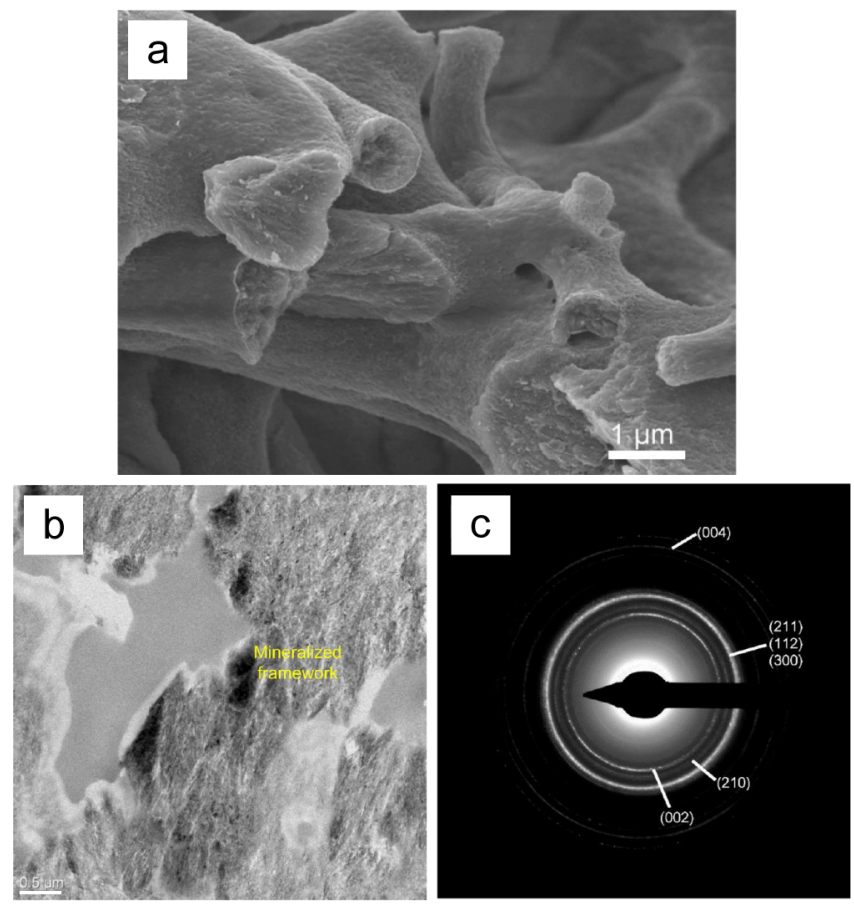

Figure 3. Intrafibrillar mineralization of an ELR sponge. (a) SEM image of an ELR sponge mineralized for 7 days, (b) brightfield TEM image of an ELR fiber mineralized for 14 days, and (c) corresponding SAED pattern showing relatively random distribution of the c-axis components 002 and 004, as well as reflections from the 211, 112, 300, and 210 planes. Reproduced with permission. ${ }^{[118]}$ Copyright 2015, American Chemical Society.

The specific advantages of the ELR system stem from their recombinant nature. The ability to engineer specific peptide sequences allows controlled design of, for example, self-assembly properties, mechanical stability, or inclusion of bioactive sequences.[119] The drawback with recombinant peptides is their cost, which currently poses a barrier to upscaling.

\subsection{Scaling up: from a building block to $2 \mathrm{D}$ and $3 \mathrm{D}$ constructs}

Organization of the building blocks into structures with different hierarchical levels is the next step in constructing a bone mimicking biomaterial/bone graft substitute. Referring to bone structure, fiber bundles and lamellae are the structures that are next on the length scale following single fibrils. At the $1-10 \mu \mathrm{m}$ 
range, the emergent characteristic of fibril organization is the parallel array of fiber bundles.

Reconstituted fibrils from diluted acidic solutions generally have a random orientation. Many different attempts have been undertaken to align the fibrils along a given direction, and more in general, to structure collagen scaffolds.[120] The approaches we will discuss here are not exhaustive; they were selected only if the following three conditions were fulfilled: 1 ) the capacity to produce collagen fibrils that retain the periodic D-spacing; 2) the ability to form 2D or 3D structures that are relevant from a bone-mimicking perspective; and 3) the applicability to either in vitro cell models or potential for upscaling to larger, implantable constructs.

Electrochemically aligned collagen fibrils can be produced by generating an electric potential difference across a dialyzed collagen solution. Using this method, fibril bundles were produced ranging from tens to hundreds of micrometers in thickness, and centimeters in length. Tropocollagen molecules align in a medium that is far from ideal for fibrillogenesis: they are at their isoelectric point (no charged residues) and there is close to zero ionic strength, due to the dialysis step. Subsequent incubation in phosphate-buffered saline is critical for allowing proper fibril formation. The process can be complemented with crosslinking. Although the characteristic D-spacing pattern could not be confirmed by SEM or TEM, the SAXS pattern showed similarity with that of a natural tendon. While this method is promising for the production of large and oriented collagen structures, more data is needed on the conservation of the native fibril conformation. ${ }^{[121,122]}$ 


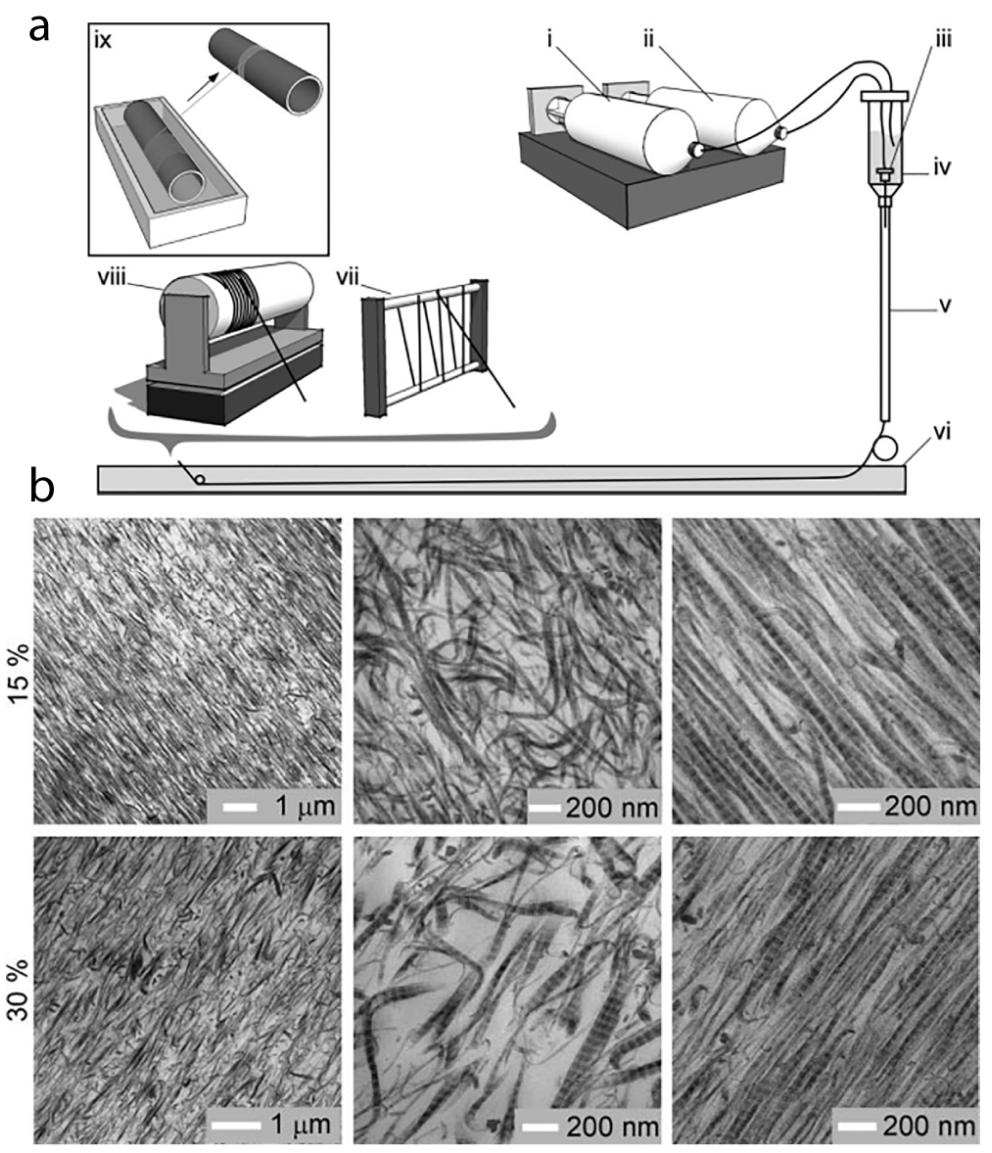

Figure 4. Wet spinning for collagen fibril alignment. (a) Schematic of the wet spinning setup, showing syringes (i,ii) with collagen and buffer solutions that are injected into a needle (iii), housed in a bubble trap (iv), with a glass capillary (v) leading to a bath containing the fiber formation buffer (vi); fibers are collected manually (vii) or automatically in a spool (viii), and finally go through a 70\% ethanol bath before being air dried (ix); (b) bright field TEM of sections of collagen fibers stained with $2 \%$ uranyl acetate after being subjected to $15 \%$ or $30 \%$ strain. While low-magnification images (left column) show highly aligned fibers, close inspection reveals in both cases areas of disorder (middle column) and order (right column) within the same fiber. Reproduced with permission. ${ }^{[123]}$ Copyright 2009, Wiley Periodicals.

A combination of wet spinning, fibrillogenesis step, and subsequent application of uniaxial strain (15\% or $30 \%$ ) produced highly aligned, elliptical-shaped fibrils, with the cross-sectional dimensions of about 50 by $20 \mu \mathrm{m}$. D-spacing of the fibrils was confirmed by TEM, although some unbanded parts were still present (Figure 4). 
This method was used to produce fibers that were hundreds of meters long.[123] Wet spinning uses a combination of solvents that is typically not compatible with the self-assembly of tropocollagen into native quarter-staggered conformation, so a second incubation step is necessary, where the drawn "amorphous" collagen fiber is immersed in a fibril formation buffer, e.g., PBS. The impact of the spinning process on the capacity of the tropocollagen molecules to assemble within the already drawn fiber is not clear, and more studies are needed to characterize the fibrillogenesis process after wet spinning. Nonetheless, this method shows great promise for scaling up: from drawn fibers of collagen into 2D sheets with control over fiber orientation, for example, by using weaving techniques and potentially into 3D scaffolds. ${ }^{[124,125]}$

Magnetic fields have also been used for aligning collagen gels, as the tropocollagen molecule has a permanent dipole, as does the aggregated fibril. A strong magnetic field (12 T) was applied to a $5 \mathrm{~mm}$ thick collagen gel $(9 \mathrm{mg} / \mathrm{mL})$ for $3 \mathrm{~h}$, resulting in alignment of about $85 \%$ of the fibers within 10 degrees of the expected direction, as was shown by $t$ SEM microscopic images. ${ }^{[126]}$ While these microscopic images only showed the gel surface, it would be interesting to investigate the alignment of the fibers in the bulk of the thick gels. Formation of fibrils with periodic D-spacing was not disturbed by the magnetic field, as shown by SEM ${ }^{[127]}$ and AFM.[126] Weaker magnetic fields were also able to induce alignment of fibrils, provided that magnetic particles (e.g., iron oxide nanoparticles) were embedded in the collagen matrix. ${ }^{[128,129]}$ However, the presence of these extra particles could hamper the mineralization process.

Using a soft lithography method microtransfer molding ( $\mu \mathrm{TM}$ ), Naik et al. were able to produce collagen patterns on flat surfaces, although with little evidence about their native conformation. ${ }^{[130]}$ Also several microfluidic-based methods were used for producing aligned collagen fibrils. A flow-assisted patterning method using parallel microchannels was used to deposit aligned fibrils. It was shown that smaller channel width led to increasing frequency of alignment, with $10 \mu \mathrm{m}$ wide channels having about $40 \%$ fibers within $5^{0}$ of the channel direction. ${ }^{[131]}$ These strategies, although useful for producing 2D substrates for studies on cell-material interaction, have limited potential for upscaling into 3D structures. A study using hydrodynamic focusing showed a great potential for continuous production of a 
collagen fiber of controllable width, in a manner similar to wet spinning, but with overall smaller fiber diameters and more control over the diameter (Figure 5). [132] Although the conditions of fibrillogenesis in these studies are amenable to formation of the native D-spacing, the data proving this were largely absent in these studies.

Apart from fibril orientation, another important characteristic for production of 2D or $3 \mathrm{D}$ collagen constructs is the packing of collagen molecules, and the density of the final material. Again, referring to the composition of natural bone, a biomimicking material should contain $\approx 65 \mathrm{wt} \%$ apatite, $\approx 25 \mathrm{wt} \%$ organic matter and a maximum of about $10 \mathrm{wt} \%$ water. Some examples of intrafibrillar mineralization studies presented in the previous section reported mineral content, as determined by TGA, of around $65 \mathrm{wt} \%{ }^{[77,80]}$ However, as most samples are lyophilized before the analysis, the reported weight percentages are those of the dry substrate.

This value is often compared directly with the inorganic content of bone cited in the literature, of $65 \mathrm{wt} \%$, without mention of the water content in the scaffold. It should be noted that the $65 \mathrm{wt} \%$ pertains to wet bone, and that the corresponding weight percentage of inorganic content in a dry sample would is about $72 \mathrm{wt} \%$. So if a mineralized and lyophilized collagen scaffold shows $65 \mathrm{wt} \%$ inorganic content, this is still very comparable to the $72 \mathrm{wt} \%$ found in bone. This means that the collagen fibrils (and not the scaffold as a whole) have a mineral content that is indeed close to that of bone fibrils. Indeed, the substrates prepared in vitro have higher porosity and take up much more than the $10 \mathrm{wt} \%$ water that bone usually contains. This becomes apparent when the mechanical properties of the biomineralized materials are measured in the wet state. Under wet conditions, the elastic modulus and hardness are generally much lower than what would be expected if the water content of such a material would be similar to that of bone. ${ }^{[80]}$ The concentrations of commercially available acidic solutions of collagen range from 2 to $10 \mathrm{mg} / \mathrm{mL}$, which is $25-125$ times lower than in natural bone. 


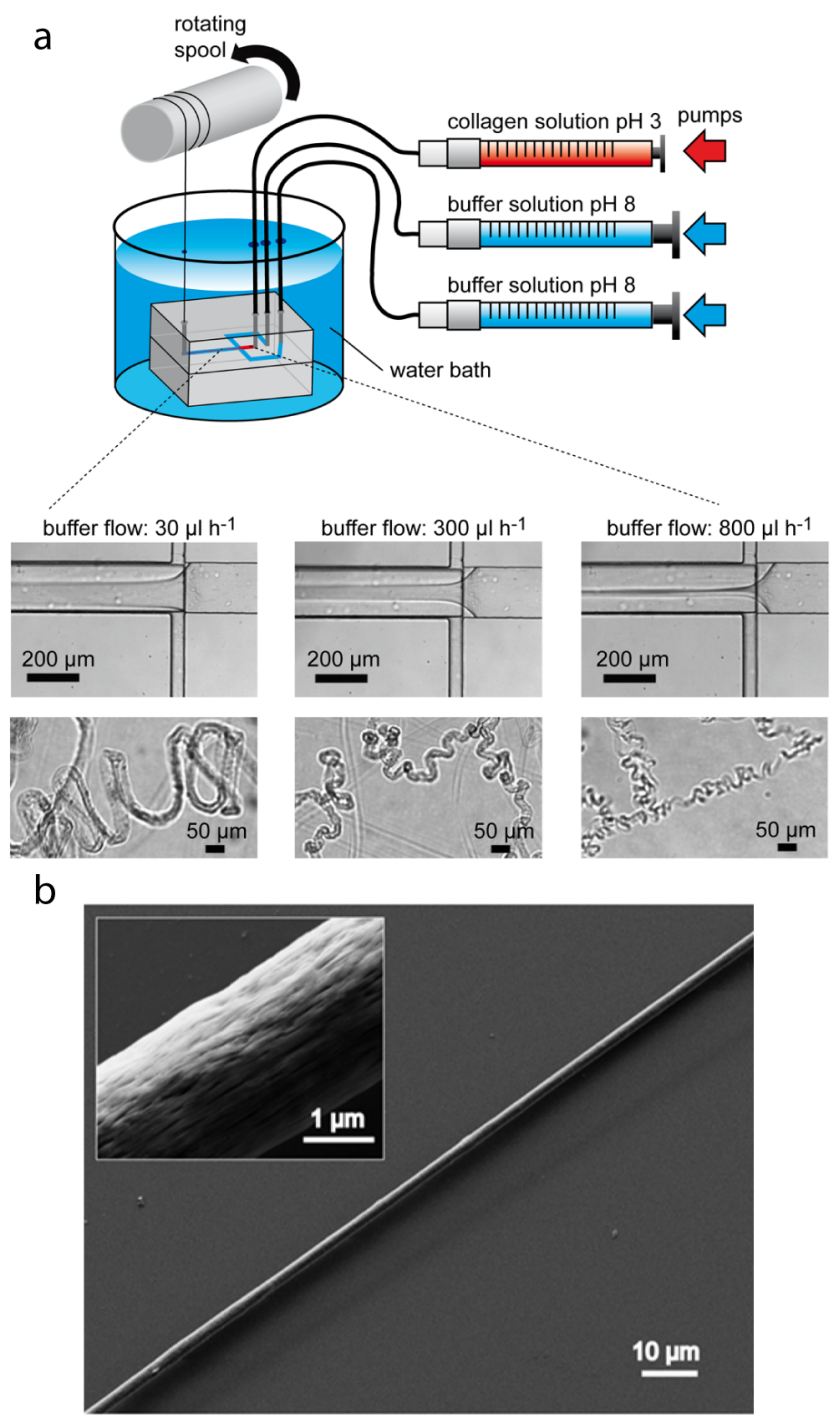

Figure 5. Microfluidic extrusion of collagen fibrils by hydrodynamic focusing. (a) Schematic of the setup, with two inlets for buffer solution (blue) and one for the collagen solution (red); the microfluidic chip is housed inside a beaker with fiber formation buffer, where the fiber passes through while being collected by a spool. This technique allows great control over fiber diameter, as seen in the images of different fiber diameter produced at different flow rates. (b) SEM image of a collagen fibril produced using this system. Reproduced with permission. ${ }^{[132]}$ Copyright 2016, American Chemical Society. 
This means that in order to have a substrate with around $10 \mathrm{wt} \%$ of water, common collagen solutions or gels have to be concentrated. A few approaches to produce dense matrices from diluted solutions of collagen have been described.

For example, based on reports that collagen exhibits a behavior similar to liquid crystals at high concentrations $(>80 \mathrm{mg} / \mathrm{mL})$, assembling into a cholesteric structure in vitro that is reminiscent of the angular step between adjacent lamella, ${ }^{[133,134]}$ Wang et al. produced organized and dense matrices from a diluted collagen solution, via a combination of injection and reverse dialysis.[135] TEM and SEM microscopic images of these dense scaffolds showed features with some similarity to osteonal bone, while collagen fibrils showed the characteristic D-spacing, confirming that they were well formed.

Due to the method used, with injection (8 days), dialysis (4 days), and fibrillogenesis (4 days), the entire process was lengthy, compounded with 16 additional days to achieve $50 \mathrm{wt} \%$ mineral content. Nevertheless, a dense scaffold was formed, showing a degree of organization that bears some resemblance to osteonal bone, which is a notable achievement.

Extensive and homogeneous mineralization of large, dense scaffolds remains a challenge, as was also illustrated by the relatively limited infiltration depth of PILP precursors in demineralized bone (up to $100 \mu \mathrm{m})^{[69]}$ and tendon tissue (up to 500 $\mu \mathrm{m}) .{ }^{[76]}$

An alternative method to obtain dense collagen scaffolds in vitro is by applying compression to typical collagen gel formulations, increasing their concentration by forcing out the water. ${ }^{[136,137]}$ This approach was used by Li et al. to obtain dense collagen matrices, in the form of a $100 \mu \mathrm{m}$ thick membrane with collagen concentration of around $80 \mathrm{mg} / \mathrm{mL}$. ${ }^{[80]}$ Homogenous mineralization by the PILP method was confirmed by SEM-EDS analysis of a cross-section of the membrane. This method yielded randomly oriented fibrils and a substrate that was less dense than the ones produced by the injection-dialysis method described above. After crosslinking and mineralization (64 wt\% dry weight), the membrane had an elastic modulus of $9 \mathrm{GPa}$ and hardness of $0.7 \mathrm{GPa}$, which were about one third of the values for lamellar bone in the dry state. When measured in the wet state, the mechanical properties decreased even further, reaching the elastic modulus of around 0.2 GPa, close to that of woven bone in the wet state. This comparison shows that, in order to mimic the mechanical properties of bone, further 
compaction of the collagen matrix, in combination with successful mineralization, is necessary.

Design of osteon-like structures that replicate its level of hierarchical organization is a difficult task to undertake. Current $3 \mathrm{D}$ printing techniques focus on the reconstruction of the central Haversian canal and network of micro channels surrounding it, typically using a biocompatible polymer like poly(lactic acid).[138] Printing techniques, however, are not capable of achieving high collagen density. An alternative approach could include a reverse-dialysis or compression method on a collagen gel containing a sacrificial framework that would be washed away in a subsequent step, creating a network of interconnected channels within a dense scaffold.

\subsection{Evaluation of bioactivity}

An important step in research and development of biomaterials for bone regeneration is the investigation of their interactions with relevant cells in vitro, such as osteoblasts, mesenchymal stem cells, etc., as a means to evaluate their bioactivity. Interestingly, only a limited number of studies exist in which collagen substrates with intrafibrillar mineralization were evaluated in vitro using cell culture systems.

In one of these studies, a crosslinked and phosphorylated collagen gel was formed on coverslips and mineralized using an SBF-like solution containing PAA. This substrate was used for culturing human periodontal ligament stem cells up to 21 days, in culture medium without stimulators of osteogenic differentiation. Controls included a collagen scaffold mineralized in the same SBF-like solution, but without PAA, as well as unmineralized collagen. Quantification of osteogenic gene expression showed upregulation of OPN, COL1A1 and BMP2 mRNA on the intrafibrillar mineralized collagen as compared to the controls. Moreover, alizarin red staining showed more pronounced mineralization on the scaffolds with intrafibrillar mineralization. ${ }^{[139]}$

In another study, an intrafibrillar-mineralized collagen scaffold was prepared using phosphorylated collagen and PAA, followed by the 21 day culture of human umbilical cord mesenchymal stem cells. Controls included collagen mineralized in the absence of PAA, non-phosphorylated mineralized collagen and unmineralized collagen. ALP activity of the cells cultured on intrafibrillar-mineralized collagen 
scaffold was higher than on the controls only after 21 days, while no differences were observed at earlier time points. These constructs were also implanted in a femoral bone defect model in rabbits. Complete defect healing after 12 weeks was only shown for the intrafibrillar-mineralized collagen, by the MRI and micro-CT analyses. ${ }^{[140]}$

A collagen scaffold with intrafibrillar mineralization, prepared with carboxymethyl chitosan as process-directing agent, was used for culturing mouse MC3T3-E1 osteoblast-like cells, for 7 days. An SBF-mineralized collagen scaffold and a collagen-only scaffold served as controls. ALP activity was higher for the scaffold with intrafibrillar mineralization after 5 and 7 days, although not at the earliest time point. These constructs were also implanted in a cranial defect model in rats, where only the intrafibrillar-mineralized scaffold showed almost complete defect closure within 8 weeks, as demonstrated by $\mu \mathrm{CT}$ and histological data.[141]

In an elegant study by Jiao et al., a biphasic silica/apatite mineralized collagen scaffold was prepared by a two-step PILP-like procedure, where PAH was used for stabilization of the silica-containing medium, and pAsp for the CaP-containing medium. Controls included a silicified-collagen substrate, a calcified-collagen substrate, and a collagen-only substrate. The in vitro cell culture was performed using mouse mesenchymal stem cells, as well as mouse macrophage cell line RAW264.7, capable of undergoing differentiation toward osteoclasts. The differentiation of mMSC in osteogenic medium, assessed by the ALP activity, was highest on the biphasic scaffold. Quantitative real-time polymerase chain reaction showed a strong effect of silica incorporation: upregulation of ALP, OPN and OPG on the one hand, and downregulation of osteoclast-activating RANKL on the other, for the biphasic and silica-collagen scaffolds. When biphasic and silica-collagen scaffolds were compared, a higher expression of the osteogenic markers was observed on the former type. The OPG upregulation and RANKL downregulation were confirmed by the Western blot analysis. Furthermore, in a coculture of RAW264.7 cells with scaffold-conditioned mMSC, it was shown that the number of TRAP-positive cells and area of resorption pits were decreased for conditions where the mMSC were cultured on silicified and biphasic scaffolds. The authors further completed the study by uncovering the signaling pathways behind the observed mechanism of inhibition of osteoclastogenesis through a series of pathway-inhibition experiments.[93] 
Another study used OPN- or pAsp-assisted mineralization of demineralized slices of bovine femur, and compared the influence of the process-directing agent on mouse osteoclasts derived from bone marrow. Controls were unaltered bone slices, as well as demineralized slices that were remineralized in the absence of OPN or pAsp. Immunofluorescence and SEM results showed, respectively, that the number of actin rings and resorption pits formed were lower on the pAspremineralized slices than on the unaltered bone slices. In contrast, the number of actin rings and resorption pits was higher when the slices were remineralized by OPN. ${ }^{[53]}$ These results suggest that OPN has a role in cell-mediated bone resorption, corroborated with evidence for less resorption by OPN-deficient mice. ${ }^{[142,143]}$

Although limited in number, the available studies on the in vitro bioactivity of intrafibrillar- mineralized collagen scaffolds show promising results, both in terms of support of differentiation of multipotent stem cells toward the osteogenic lineage, as well as for being able to be resorbed by cell-mediated phenomena. The two in vivo experiments ${ }^{[140,141]}$ reviewed also show promising results, with better healing of bone defects when compared to the respective controls.

Continued biological experiments to evaluate the bioactivity of bone-mimicking substitutes are a necessity, to validate material design and investigate whether pursuit of the biomimetic route indeed delivers on its promises.

\subsection{Translation to the clinic}

Some efforts have been made to translate the amorphous precursor systems into clinical strategies for remineralization of hypo-mineralized tissues, as solutionbased systems have limitations for application in a clinical situation. Typically these efforts involve the use of a carrier system to deliver the mineralizing components to the target tissue. For example, mesoporous silica nanoparticles were loaded with PAA-stabilized ACP, and used for mineralizing reconstituted collagen fibrils in vitro. TEM analysis showed the presence of intrafibrillar mineral, throughout the collagen network, after 4 days. [144,145]

Another example is the use of self-etch adhesives, currently used in the treatment of dental cavities, as a carrier system for pAsp-stabilized silica-doped ACP. When tested on demineralized dentin, the adhesives produced a 1-2 $\mu$ m layer of lightly re-mineralized tissue after 14 days, close to the native dentin. [146] 
These examples show a simple and effective way of translating an in vitro concept of biomineralization directly to a clinical setting, and are a promising first step toward developing biomimetic remineralization systems. The current challenges are related with volume of mineralizing components that can be delivered; the adhesive mentioned above was loaded with $25 \mathrm{wt} \%$ of amorphous precursor particles, which could potentially be increased, but not by a large amount. Alternative strategies could focus on using the mineral ions present in surrounding physiological fluids as the source for continued mineralization. 


\section{Conclusion and Outlook}

Successful intrafibrillar mineralization of collagen substrates in vitro, achieved in the past decade, has been an important step forward toward truly biomimetic bone-like structures. As is evident from the different studies reviewed here, the lab-made building blocks closely replicate some of the most important features found natural tissue, such as mineral density, particle size, and crystallite orientation. Nevertheless, for some other characteristics, it is still unclear to which extent the in vitro built structures resemble their natural counterparts. For example, more characterization of the chemical identity of the in vitro formed intrafibrillar mineral is required, in particular regarding the substitutions by ions present in the mineralizing solution. This could deliver further insights into the mechanism of polymer-assisted mineralization as some ions were shown to disrupt the process. Furthermore, while present in low (often trace) amounts in natural bone mineral, the relevance of these ions should not be ignored; on the contrary, bioinorganic additives to the mineral may enhance the bone regenerative potential to the biomimetic bone graft substitutes.

This review has also shown the importance of advanced analytical techniques in understanding the natural process of biomineralization, which in turn should be applied to the design and development of new biomimetic biomaterials. For example, the curved needle-like crystallites, shown by 3D reconstruction of HRTEM of bone slices to interpenetrate more than one collagen fibril, ${ }^{[31]}$ put in question the division between intrafibrillar and extrafibrillar mineral and beg for new hypothesizes regarding the formation of these structures. Furthermore, continued research toward full understanding of the roles of NCPs in bone mineralization might shed light upon the crystallization process of these particles. Research into and development of (synthetic) alternatives to collagen as structural component of the ECM is also highly important for two reasons. First, the use of synthetic, often well-characterized materials may contribute to the knowledge of the fundamentals of biomineralization, for example, regarding the role of NCPs, by allowing a direct comparison with collagen. Second, the development of collagen alternatives is highly important from a translational perspective, where the use of a synthetic material has several advantages over the (xenogeneic) collagen. The only system that has shown convincing intrafibrillar-like mineralization is based on recombinant proteins, which add versatility and customization features, but are 
expensive for upscaling. Therefore, efforts to develop other alternative matrices that can overcome this issue are justified. As exemplified by the ELRs, and also by a study on mineralization upon physical confinement, ${ }^{[147]}$ a critical feature for intrafibrillar-like mineralization seems to be the existence of stable, structural confined spaces. This is achieved in both collagen and ELR by the assembly of small building blocks, of tropocollagen and $\beta$-spirals, respectively, into larger hierarchical structures - collagen fibrils (staggered tropocollagen molecules) and ELR filaments (association of folded $\beta$-spirals). It is envisioned that by this same principle of producing stable molecular structures with nanoporosity, other polymeric systems can be used for intrafibrillar-like mineralization.

Upscaling from a building block into organized 2D and 3D assemblies comes with two main challenges: directionality of the collagen fibers and compact arrangement into dense substrates.

State-of-the-art methodologies capable of aligning collagen fibrils while retaining their capacity for bone-like mineralization come from many different fields. Of note are wetspinning ${ }^{[123]}$ and microfluidic ${ }^{[132]}$ approaches, that have the potential to produce aligned bundles of fibers with the native D-spacing, as well as the advantage of having some control over fiber diameter.

These can be combined with other techniques, for example weaving of biopolymers, ${ }^{[125]}$ to fabricate $2 \mathrm{D}$ bidirectional meshes of parallel collagen fibers. Control over the fibril thickness and pore dimension, and further stacking of 2D meshes would result in a 3D structure structurally resembling cancellous bone.

It should be emphasized that one of the main reasons for pursuing the biomimetic approaches to develop synthetic bone graft substitutes is the potential to match the mechanical characteristics of natural bone, which cannot be achieved with currently widely used $(\mathrm{CaP})$ ceramic bone graft substitutes, which are intrinsically brittle. Interestingly, in many studies reviewed here, the mechanical properties have not been extensively evaluated, while specific mechanical tests would be useful, for example, in determining the importance of fibril alignment.

It has been shown that the elastic modulus of synthetic scaffolds is about $33 \%$ (for ELR) and $13 \%$ (for collagen) of that of lamellar bone. This difference can be attributed to an insufficient fibril density of the synthetic scaffolds, which generally perform poorly when in the wet state. Replication of the cortical bone density requires the mineralization of large collagen substrates with limited porosity 
$(\approx 6 \%)$, which is a challenging task. The study by Wang et al. is a good example of exploiting the liquid-crystal behavior of collagen solutions, at high concentrations, to form dense collagen substrates that also show some degree of fiber orientation. ${ }^{[66]}$ Another promising method is the use of plastic compression applied to gels with prealigned fibrils, obtained, for example, using magnetic fields.

While 3D constructs are essential from a translational/clinical perspective, planar structures are a necessary step in getting there, meanwhile being useful for cellmaterial interaction studies that provide data to sustain further developments of biomimetic materials. As discussed in the last section of the review, the number of studies dealing with the interactions between relevant cells and the latest wave of biomimetic materials for bone regeneration is limited. Nevertheless, the results of these studies are compelling. In particular, further studies concentrating on the interplay between osteoblasts and osteoclasts would be useful in establishing a model for resorption and replacement of these synthetic substrates by natural bone ECM.

It must be noted that the reason for choosing a biomimetic route to biomaterial design and development does not come solely from its potential to deliver better materials for the clinic. Indeed, no consensus in the field of materials for biomedical applications exists that biomimetic or bioinspired methods are more promising than other methods for developing successful treatments of damaged and diseased organs and tissues. Nevertheless, by using more natural-like strategies in vitro, it is sometimes possible to link laboratory evidence to actual in vivo mechanisms, as was the case for the discovery of amorphous precursors in vertebrate bone. The combination of fundamental and applied studies that take place at the biomimetic border fuel each other into new discoveries, and this is one of the main strengths of biomimetism. 


\section{Funding}

This research has been made possible with the support of the Dutch Province of Limburg.

\section{Conflict of interest}

The authors confirm that there are no known conflicts of interest associated with this publication and there has been no significant financial support for this work that could have influenced its outcome. 


\section{References}

[1] V. Campana, G. Milano, E. Pagano, M. Barba, C. Cicione, G. Salonna, W. Lattanzi, G. Logroscino, J. Mater. Sci. Mater. Med. 2014, 25, 2445.

[2] S. N. Khan, F. P. Cammisa, H. S. Sandhu, A. D. Diwan, F. P. Girardi, J. M. Lane, J. Am. Acad. Orthop. Surg. 2005, 13, 77.

[3] R. Dimitriou, G. I. Mataliotakis, A. G. Angoules, N. K. Kanakaris, P. V. Giannoudis, Injury 2011, 42, S3.

[4] T. Kurien, R. G. Pearson, B. E. Scammell, Bone Jt. J. 2013, 95 B, 583.

[5] D. Salem, Z. Natto, S. Elangovan, N. Karimbux, J. Periodontol. 2016, 87, 872.

[6] J.-H. Zeng, S.-W. Liu, L. Xiong, P. Qiu, L.-H. Ding, S.-L. Xiong, J.-T. Li, X.-G. Liao, Z.-M. Tang, J. Orthop. Surg. Res. 2018, 13, 33.

[7] D. Rickert, J. J. R. H. Slater, H. J. A. Meijer, A. Vissink, G. M. Raghoebar, Int. J. Oral Maxillofac. Surg. 2012, 41, 160.

[8] R. Agarwal, K. Williams, C. A. Umscheid, W. C. Welch, J. Neurosurg. Spine 2009, 11, 729.

[9] D. M. Fisher, J. M.-L. Wong, C. Crowley, W. S. Khan, Curr. Stem Cell Res. Ther. 2013, $8,260$.

[10] A. Moya, J. Paquet, M. Deschepper, N. Larochette, K. Oudina, C. Denoeud, M. Bensidhoum, D. Logeart-Avramoglou, H. Petite, Stem Cells 2018, 36, 363.

[11] W. Wang, K. W. K. Yeung, Bioact. Mater. 2017, 2, 224.

[12] A. W. Ritting, E. W. Weber, M. C. Lee, J. Hand Surg. Am. 2012, 37, 316.

[13] S. Boraiah, O. Paul, D. Hawkes, M. Wickham, D. G. Lorich, Clin. Orthop. Relat. Res. 2009, 467, 3257.

[14] H. S. Johal, R. E. Buckley, I. L. D. Le, R. K. Leighton, J. Trauma Acute Care Surg. 2009, 67,875 .

[15] T. A. Russell, R. K. Leighton, JBJS 2008, 90, 2057.

[16] H. Bae, H. P. J. Hatten, R. Linovitz, A. D. Tahernia, M. K. Schaufele, V. McCollom, L. Gilula, P. Maurer, R. Benyamin, J. M. Mathis, M. Persenaire, Spine 2012, 37, 544.

[17] T. Lerner, V. Bullmann, T. L. Schulte, M. Schneider, U. Liljenqvist, Eur. Spine J. 2009, 18,170 .

[18] C. Cassidy, J. B. Jupiter, M. Cohen, M. Delli-Santi, C. Fennell, C. Leinberry, J. Husband, A. Ladd, W. R. Seitz, B. Constanz, J. Bone Joint Surg. Am. 2003, 85-A, 2127.

[19] M. Bohner, L. Galea, N. Doebelin, J. Eur. Ceram. Soc. 2012, 32, 2663.

[20] M. Granke, M. D. Does, J. S. Nyman, Calcif. Tissue Int. 2015, 97, 292.

[21] P. Fratzl, R. Weinkamer, Prog. Mater. Sci. 2007, 52, 1263.

[22] S. P. Robins, J. D. Brady, in Principles of Bone Biology, Vol. 1 (Eds: J.P. Bilezikian, L.G. Raisz, T.J. Martin), Academic Press, Massachusetts, USA 2008, Ch. 16.

[23] M. Saito, K. Marumo, Calcif. Tissue Int. 2015, 97, 242.

[24] J. R. Harris, A. Soliakov, R. J. Lewis, Micron 2013, 49, 60.

[25] W. Zhu, P. G. Robey, A. L. Boskey, in Osteoporosis, Vol. 1 (Eds: R. Marcus, D. Feldman, D. Nelson, C. Rosen), Academic Press, Massachusets, USA 2008, Ch. 9.

[26] P. J. Thurner, C. G. Chen, S. Ionova-Martin, L. Sun, A. Harman, A. Porter, J. W. 
Ager, R. O. Ritchie, T. Alliston, Bone 2010, 46, 1564.

[27] A. A. Poundarik, T. Diab, G. E. Sroga, A. Ural, A. L. Boskey, C. M. Gundberg, D. Vashishth, Proc. Natl. Acad. Sci. 2012, 109, 19178.

[28] E. A. McNally, H. P. Schwarcz, G. A. Botton, A. L. Arsenault, PLoS One 2012, 7, DOI: 10.1371/journal.pone.0029258.

[29] E. McNally, F. Nan, G. A. Botton, H. P. Schwarcz, Micron 2013, 49, 46.

[30] M. J. Olszta, X. Cheng, S. S. Jee, R. Kumar, Y. Y. Kim, M. J. Kaufman, E. P. Douglas, L. B. Gower, Mater. Sci. Eng. R Reports 2007, 58, 77.

[31] N. Reznikov, M. Bilton, L. Lari, M. M. Stevens, R. Kröger, Science 2018, 360, DOI: 10.1126/science.aao2189.

[32] B. Wopenka, J. D. Pasteris, Mater. Sci. Eng. C 2005, 25, 131.

[33] R. K. Rude, in Principles of Bone Biology, Vol. 1 (Eds: J.P. Bilezikian, L.G. Raisz, T.J. Martin), Academic Press, Massachusetts, USA 2008, Ch. 24.

[34] E. Boanini, M. Gazzano, A. Bigi, Acta Biomater. 2010, 6, 1882.

[35] F. Bronner, in Principles of Bone Biology, Vol. 1 (Eds: J.P. Bilezikian, L.G. Raisz, T.J. Martin), Academic Press, Massachusetts, USA 2008, Ch. 25.

[36] O. A. Golovanova, N. N. Strunina, S. A. Lemesheva, B. T. Baisova, J. Appl. Spectrosc. 2011, 78, 145.

[37] N. P. Zaksas, T. T. Sultangazieva, V. A. Gerasimov, Anal. Bioanal. Chem. 2008, 391, 687.

[38] G. Ma, X. Y. Liu, Cryst. Growth Des. 2009, 9, 2991.

[39] L. C. Costello, M. Chellaiah, J. Zou, R. B. Franklin, M. A. Reynolds, J. Regen. Med. Tissue Eng. 2014, 3, 4.

[40] Y.-Y. Hu, A. Rawal, K. Schmidt-Rohr, Proc. Natl. Acad. Sci. 2010, 107, 22425.

[41] E. Davies, K. H. Muller, W. C. Wong, C. J. Pickard, D. G. Reid, J. N. Skepper, M. J. Duer, Proc. Natl. Acad. Sci. 2014, 111, DOI: 10.1073/pnas.1315080111.

[42] J. M. Delgado-López, F. Bertolotti, J. Lyngsø, J. S. Pedersen, A. Cervellino, N. Masciocchi, A. Guagliardi, Acta Biomater. 2017, 49, 555.

[43] J. Mahamid, A. Sharir, L. Addadi, S. Weiner, Proc. Natl. Acad. Sci. 2008, 105, 12748.

[44] M. Bennet, A. Akiva, D. Faivre, G. Malkinson, K. Yaniv, S. Abdelilah-Seyfried, P. Fratzl, A. Masic, Biophys. J. 2014, 106, L17.

[45] J. Mahamid, B. Aichmayer, E. Shimoni, R. Ziblat, C. Li, S. Siegel, O. Paris, P. Fratzl, S. Weiner, L. Addadi, Proc. Natl. Acad. Sci. 2010, 107, 6316.

[46] J. Mahamid, A. Sharir, D. Gur, E. Zelzer, L. Addadi, S. Weiner, J. Struct. Biol. 2011, $174,527$.

[47] N. J. Crane, V. Popescu, M. D. Morris, P. Steenhuis, M. A. Ignelzi, Bone 2006, 39, 434.

[48] E. Beniash, R. A. Metzler, R. S. K. Lam, P. U. P. A. Gilbert, J. Struct. Biol. 2009, 166, 133.

[49] J. J. De Yoreo, P. U. P. A. Gilbert, N. A. J. M. Sommerdijk, R. L. Penn, S. Whitelam, D. Joester, H. Zhang, J. D. Rimer, A. Navrotsky, J. F. Banfield, A.F. William, F.M. Michel, F.C. Meldrum, H. Cölfen, P.M. Dove, Science 2015, 349, DOI: 10.1126/science.aaa6760.

[50] W. J. E. M. Habraken, J. Tao, L. J. Brylka, H. Friedrich, L. Bertinetti, A. S. Schenk, A. Verch, V. Dmitrovic, P. H. H. Bomans, P. M. Frederik, J. Laven, P. van der Schoot, B. 
Aichmayer, G. de With, J.J. De Yoreo, N. Sommerdijk, Nat. Commun. 2013, 4, 1507.

[51] A. S. Deshpande, P. A. Fang, X. Zhang, T. Jayaraman, C. Sfeir, E. Beniash, Biomacromolecules 2011, 12, 2933.

[52] F. Nudelman, P. H. H. Bomans, A. George, G. de With, N. A. J. M. Sommerdijk, Faraday Discuss. 2012, 159, 357.

[53] D. E. Rodriguez, T. Thula-Mata, E. J. Toro, Y. W. Yeh, C. Holt, L. S. Holliday, L. B. Gower, Acta Biomater. 2014, 10, 494.

[54] D. Toroian, E. L. Joo, P. A. Price, J. Biol. Chem. 2007, 282, 22437.

[55] P. A. Price, D. Toroian, J. E. Lim, J. Biol. Chem. 2009, 284, 17092.

[56] F. Nudelman, K. Pieterse, A. George, P. H. H. Bomans, H. Friedrich, L. J. Brylka, P. A. J. Hilbers, G. De With, N. A. J. M. Sommerdijk, Nat. Mater. 2010, 9, 1004.

[57] A. J. Lausch, E. D. Sone, Biomacromolecules 2015, 16, 1938.

[58] W. E. G. Müller, H. C. Schröder, U. Schlossmacher, V. A. Grebenjuk, H. Ushijima, X. Wang, Biomaterials 2013, 34, 8671.

[59] X. Wang, H. C. Schröder, U. Schlossmacher, M. Neufurth, Q. Feng, B. Diehl-Seifert, W. E. G. Müller, Calcif. Tissue Int. 2014, 94, 495.

[60] K. Nitiputri, Q. M. Ramasse, H. Autefage, C. M. McGilvery, S. Boonrungsiman, N. D. Evans, M. M. Stevens, A. E. Porter, ACS Nano 2016, 10, 6826.

[61] K. Jiao, L. Niu, C. F. Ma, X. Q. Huang, D. D. Pei, T. Luo, Q. Huang, J. H. Chen, F. R. Tay, Adv. Funct. Mater. 2016, 26, 6858.

[62] J. Mitchell, A. H. van Heteren, Comptes Rendus - Palevol 2016, 15, 23.

[63] T. G. Bromage, H. M. Goldman, S. C. McFarlin, J. Warshaw, A. Boyde, C. M. Riggs, Anat. Rec. 2003, 274B, 157.

[64] T. Yamamoto, T. Hasegawa, M. Sasaki, H. Hongo, C. Tabata, Z. Liu, M. Li, N. Amizuka, J. Electron Microsc. 2012, 61, 113.

[65] L. B. Gower, D. J. Odom, J. Cryst. Growth 2000, 210, 719.

[66] Y. Wang, T. Azaïs, M. Robin, A. Vallée, C. Catania, P. Legriel, G. Pehau-Arnaudet, F. Babonneau, M. M. Giraud-Guille, N. Nassif, Nat. Mater. 2012, 11, 724.

[67] A. Akiva, G. Malkinson, A. Masic, M. Kerschnitzki, M. Bennet, P. Fratzl, L. Addadi, S. Weiner, K. Yaniv, Bone 2015, 75, 192.

[68] D. Kim, B. Lee, S. Thomopoulos, Y.-S. Jun, Cryst. Growth Des. 2016, 16, 5359.

[69] T. T. Thula, D. E. Rodriguez, M. H. Lee, L. Pendi, J. Podschun, L. B. Gower, Acta Biomater. 2011, 7, 3158.

[70] A. K. Burwell, T. Thula-Mata, L. B. Gower, S. Habeliz, M. Kurylo, S. P. Ho, Y. C. Chien, J. Cheng, N. F. Cheng, S. A. Gansky, S.J. Marshall, W. Grayson, PLoS One 2012, 7, DOI: 10.1371/journal.pone.0038852.

[71] T. Thula-Mata, A. Burwell, L. B. Gower, S. Habelizt, G. Marshall, Mater. Res. Soc. Symp. Proc. 2011, 1355, DOI: 10.1557/opl.2011.1114.

[72] A. J. Lausch, B. D. Quan, J. W. Miklas, E. D. Sone, Adv. Funct. Mater. 2013, 23, 4906.

[73] H. Nurrohman, K. Saeki, K. Carneiro, Y. C. Chien, S. Djomehri, S. P. Ho, C. Qin, S. J. Marshall, L. B. Gower, G. W. Marshall, J. Mater. Res. 2016, 31, 321.

[74] H. Nurrohman, K. M. M. Carneiro, J. Hellgeth, K. Saeki, S. J. Marshall, G. W. Marshall, S. Habelitz, PLoS One 2017, 12, DOI: 10.1371/journal.pone.0188277.

[75] A. S. Deshpande, E. Beniash, Cryst. Growth Des. 2008, 8, 3084. 
[76] S. Jee, L. Culver, Y. Li, E. P. Douglas, L. B. Gower, J. Cryst. Growth 2010, 312, 1249.

[77] S. Jee, T. T. Thula, L. B. Gower, Acta Biomater. 2010, 6, 3676.

[78] S. S. Jee, R. K. Kasinath, E. DiMasi, Y.-Y. Kim, L. Gower, CrystEngComm 2011, 13, 2077.

[79] T. T. Thula, F. Svedlund, D. E. Rodriguez, J. Podschun, L. Pendi, L. B. Gower, Polym. Basel 2011, 3, 10.

[80] Y. Li, T. T. Thula, S. Jee, S. L. Perkins, C. Aparicio, E. P. Douglas, L. B. Gower, Biomacromolecules 2011, 13, 49.

[81] Y. K. Kim, L. S. Gu, T. E. Bryan, J. R. Kim, L. Chen, Y. Liu, J. C. Yoon, L. Breschi, D. H. Pashley, F. R. Tay, Biomaterials 2010, 31, 6618.

[82] J. Chen, C. Burger, C. V. Krishnan, B. Chu, B. S. Hsiao, M. J. Glimcher, Macromol. Chem. Phys. 2005, 206, 43.

[83] K. Liang, Y. Gao, J. Lie, Y. Liao, S. Xiao, X. Zhou, J. Li, J. Biomater. Sci. Polym. Ed. 2015, 26, 963.

[84] S. Tao, M. Fan, H. H. K. Xu, J. Li, L. He, RSC Adv. 2017, 7, 54947.

[85] Y. Liu, N. Li, Y. P. Qi, L. Dai, T. E. Bryan, J. Mao, D. H. Pashley, F. R. Tay, Adv. Mater. 2011, 23, 975.

[86] F. R. Tay, D. H. Pashley, Biomaterials 2008, 29, 1127.

[87] L. S. Gu, J. Kim, Y. K. Kim, Y. Liu, S. H. Dickens, D. H. Pashley, J. Q. Ling, F. R. Tay, Dent. Mater. 2010, 26, 1077.

[88] J. Sun, C. Chen, H. Pan, Y. Chen, C. Mao, W. Wang, R. Tang, X. Gu, J. Mater. Chem. B 2014, 2, 4544.

[89] L. Gu, Y. K. Kim, Y. Liu, H. Ryou, C. E. Wimmer, L. Dai, D. D. Arola, S. W. Looney, D. H. Pashley, F. R. Tay, J. Dent. Res. 2011, 90, 82.

[90] H. Nurrohman, S. Nakashima, T. Takagaki, A. Sadr, T. Nikaido, Y. Asakawa, M. Uo, S. J. Marshall, J. Tagami, Biomed. Mater. Eng. 2015, 25, 89.

[91] L. Niu, S. E. Jee, K. Jiao, L. Tonggu, M. Li, L. Wang, Y. D. Yang, J. H. Bian, L. Breschi, S. S. Jang, J.H. Chen, D.H. Pashley, F.R. Tay, Nat. Mater. 2017, 16, 370.

[92] L. N. Niu, K. Jiao, H. Ryou, C. K. Y. Yiu, J. H. Chen, L. Breschi, D. D. Arola, D. H. Pashley, F. R. Tay, Angew. Chemie - Int. Ed. 2013, 52, 5762.

[93] K. Jiao, L. Niu, Q. Li, F. Chen, W. Zhao, J. Li, J. H. Chen, C. W. Cutler, D. H. Pashley, F. R. Tay, Acta Biomater. 2015, 19, 23.

[94] L. Kalmar, D. Homola, G. Varga, P. Tompa, Bone 2012, 51, 528.

[95] A. George, A. Veis, Chem. Rev. 2009, 108, 4670.

[96] L. B. Gower, in Biomineralization and Biomaterials: Fundamentals and Applications (Eds: C. Aparicio, M.P. Ginebra) Woodhead Publishing, Cambridge, United Kingdom 2015, Ch. 6.

[97] C. Shao, R. Zhao, S. Jiang, S. Yao, Z. Wu, B. Jin, Y. Yang, H. Pan, R. Tang, Adv. Mater. 2018, 30, 1 .

[98] K. Iijima, M. Hashizume, Protein Pept. Lett. 2018, 25, 25.

[99] C. Sfeir, P.-A. Fang, J. Thottala, A. Raman, Z. Xiaoyuan, E. Beniash, Acta Biomater. 2014, 10, 2241.

[100] H. Ping, H. Xie, B. Su, Y. Cheng, W. Wang, H. Wang, Y. Wang, J. Zhang, F. Zhang, Z. Fu, J. Mater. Chem. B 2015, 3, 4496. 
[101] J. H. Shepherd, D. V Shepherd, S. M. Best, J. Mater. Sci. Med. 2012, 23, 2335.

[102] M. Šupová, Ceram. Int. 2015, 41, 9203.

[103] A. Malhotra, P. Habibovic, Trends Biotechnol. 2016, 34, 983.

[104] S. Bose, G. Fielding, S. Tarafder, A. Bandyopadhyay, Trends Biotechnol. 2013, 31, 594.

[105] C. Rey, B. Collins, T. Goehl, I. R. Dickson, M. J. Glimcher, Calcif. Tissue Int. 1989, 45, 157.

[106] F. J. Martínez-Casado, M. Iafisco, J. M. Delgado-López, C. Martínez-Benito, C. RuizPérez, D. Colangelo, F. Oltolina, M. Prat, J. Gómez-Morales, Cryst. Growth Des. 2016, 16, 145.

[107] S. Lala, M. Ghosh, P. K. Das, D. Das, T. Kar, S. K. Pradhan, Mater. Chem. Phys. 2016, 170, 319 .

[108] D. Laurencin, M. E. Smith, Prog. Nucl. Magn. Reson. Spectrosc. 2013, 68, DOI: 10.1016/j.pnmrs.2012.05.001.

[109] A. Bigi, M. Debbabi, M. Gazzano, N. Roveri, Eur. J. Inorg. Chem. 2001, 5, 1261.

[110] S. Caldarelli, J. Eon, D. Laurencin, M. E. Smith, J. Am. Chem. Soc. 2009, 131, 5145.

[111] C. Li, O. Paris, S. Siegel, P. Roschger, E. P. Paschalis, K. Klaushofer, P. Fratzl, J. Bone Miner. Res. 2010, 25, 968.

[112] A. Henss, A. Hild, M. Rohnke, S. Wenisch, J. Janek, Biointerphases 2016, 11, DOI: 10.1116/1.4928211.

[113] M. Rohnke, A. Henss, J. Kokesch-Himmelreich, M. Schumacher, S. Ray, V. Alt, M. Gelinsky, J. Janek, Anal. Bioanal. Chem. 2013, 405, 8769.

[114] L. M. Gordon, L. Tran, D. Joester, ACS Nano 2012, 6, 10667.

[115] C. Capuccini, P. Torricelli, E. Boanini, M. Gazzano, R. Giardino, A. Bigi, J. Biomed. Mater. Res. - Part A 2009, 89, 594.

[116] Z. T. Birgani, A. Malhotra, C. A. van Blitterswijk, P. Habibovic, J. Biomed. Mater. Res. - Part A 2016, 104, 1946.

[117] J. Barralet, U. Gbureck, P. Habibovic, E. Vorndran, C. Gerard, C. J. Doillon, Tissue Eng. Part A 2009, 15, 1601.

[118] Y. Li, X. Chen, A. Fok, J. C. Rodriguez-Cabello, C. Aparicio, ACS Appl. Mater. Interfaces 2015, 7, 25784.

[119] Y. Li, J. C. Rodriguez-Cabello, C. Aparicio, ACS Appl. Mater. Interfaces 2017, 9, 5838.

[120] B. D. Walters, J. P. Stegemann, Acta Biomater. 2014, 10, 1488.

[121] X. Cheng, U. A. Gurkan, C. J. Dehen, M. P. Tate, H. W. Hillhouse, G. J. Simpson, O. Akkus, Biomaterials 2008, 29, 3278.

[122] J. A. Uquillas, V. Kishore, O. Akkus, Biomed. Mater. 2011, 6, DOI 10.1088/17486041/6/3/035008.

[123] J. M. Caves, V. A. Kumar, J. Wen, W. Cui, A. Martinez, R. Apkarian, J. E. Coats, K. Berland, E. L. Chaikof, J. Biomed. Mater. Res. - Part B Appl. Biomater. 2010, 93, 24.

[124] J. L. Ng, L. E. Knothe, R. M. Whan, U. Knothe, M. L. K. Tate, Sci. Rep. 2017, 7, DOI: 10.1038/srep40396.

[125] J. Gilmore, T. Burg, R. E. Groff, K. J. L. Burg, J. Biomed. Mater. Res. - Part B Appl. Biomater. 2017, 105, 1342.

[126] S. Chen, N. Hirota, M. Okuda, M. Takeguchi, H. Kobayashi, N. Hanagata, T. Ikoma, Acta Biomater. 2011, 7, 644. 
[127] J. Torbet, M. Malbouyres, N. Builles, V. Justin, M. Roulet, O. Damour, A. Oldberg, F. Ruggiero, D.J. Hulmes, Biomaterials 2007, 28, 4268.

[128] C. Guo, L. J. Kaufman, Biomaterials 2007, 28, 1105.

[129] J. Zhuang, S. Lin, L. Dong, K. Cheng, W. Weng, ACS Biomater. Sci. Eng. 2018, 4, 1528.

[130] N. Naik, J. Caves, E. L. Chaikof, M. G. Allen, Adv. Healthc. Mater. 2014, 3, 367.

[131] P. Lee, R. Lin, J. Moon, L. P. Lee, Biomed. Microdevices 2006, 8, 35.

[132] C. Haynl, E. Hofmann, K. Pawar, S. Förster, T. Scheibel, Nano Lett. 2016, 16, 5917.

[133] M. M. Giraud-Guille, G. Mosser, E. Belamie, Curr. Opin. Colloid Interface Sci. 2008, 13, 303.

[134] M. M. Giraud Guille, G. Mosser, C. Helary, D. Eglin, Micron 2005, 36, 602.

[135] Y. Wang, J. Silvent, M. Robin, F. Babonneau, A. Meddahi-Pellé, N. Nassif, M. M. Giraud Guille, Soft Matter 2011, 7, 9659.

[136] R. A. Brown, M. Wiseman, C. B. Chuo, U. Cheema, S. N. Nazhat, Adv. Funct. Mater. 2005, 15, 1762.

[137] E. A. Abou Neel, U. Cheema, J. C. Knowles, R. A. Brown, S. N. Nazhat, Soft Matter 2006, 2, 986.

[138] H. Cui, W. Zhu, M. Nowicki, X. Zhou, A. Khademhosseini, L. G. Zhang, Adv. Healthc. Mater. 2016, 5, 2174.

[139] Y. Fu, S. Liu, S. Cui, X. Kou, X. Wang, X. Liu, Y. Sun, G. Wang, Y. Liu, Y. Zhou, ACS Appl. Mater. Interfaces 2016, 8, 15958.

[140] B. Ye, X. Luo, Z. Li, C. Zhuang, L. Li, L. Lu, S. Ding, J. Tian, C. Zhou, Mater. Sci. Eng. C 2016, 68, 43.

[141] Y. Wang, N. Van Manh, H. Wang, X. Zhong, X. Zhang, C. Li, Int. J. Nanomedicine 2016, 11, 2053.

[142] C. L. Duvall, W. R. Taylor, D. Weiss, A. M. Wojtowicz, R. E. Guldberg, J. Bone Miner. Res. 2007, 22, 286.

[143] A. Franzén, K. Hultenby, F. P. Reinholt, P. Önnerfjord, D. Heinegård, J. Orthop. Res. 2008, 26, 721.

[144] X. J. Luo, H. Y. Yang, L. N. Niu, J. Mao, C. Huang, D. H. Pashley, F. R. Tay, Acta Biomater. 2016, 31, 378.

[145] W. Zhang, X. J. Luo, L. N. Niu, H. Y. Yang, C. K. Y. Yiu, T. Da Wang, L. Q. Zhou, J. Mao, C. Huang, D. H. Pashley, F.R. Tay, Sci. Rep. 2015, 5, DOI: 10.1038/srep11199.

[146] Z. Wu, X. Wang, Z. Wang, C. Shao, X. Jin, L. Zhang, H. Pan, R. Tang, B. Fu, ACS Appl. Mater. Interfaces 2017, 9, 17710.

[147] B. Cantaert, E. Beniash, F. C. Meldrum, Chemistry 2013, 19, 14918.

[148] U. G. K. Wegst, H. Bai, E. Saiz, A. P. Tomsia, R. O. Ritchie, Nat. Mater. 2015, 14, 23.

[149] M. Nakamura, S. Sone, I. Takahashi, I. Mizoguchi, S. Echigo, Y. Sasano, J. Histochem. Cytochem. 2005, 53, 1553.

[150] L. J. Foster, P. A. Zeemann, C. Li, M. Mann, O. N. Jensen, M. Kassem, Stem Cells 2005, 23, 1367.

[151] W. W. Du, L. Fang, W. Yang, W. Sheng, Y. Zhang, A. Seth, B. B. Yang, A. J. Yee, BMC Cancer 2012, 12, 341.

[152] I. Zvackova, E. Matalova, H. Lesot, Front. Physiol. 2017, 8, DOI: 10.3389/fphys.2017.00554. 
[153] T. Douglas, S. Heinemann, S. Bierbaum, D. Scharnweber, H. Worch, Biomacromolecules 2006, 7, 2388.

[154] Y. Mochida, D. Parisuthiman, S. Pornprasertsuk-Damrongsri, P. Atsawasuwan, M. Sricholpech, A. L. Boskey, M. Yamauchi, Matrix Biol. 2009, 28, 44.

[155] A. D. Berendsen, E. L. Pinnow, A. Maeda, A. C. Brown, N. McCartney-Francis, V. Kram, R. T. Owens, P. G. Robey, K. Holmbeck, L. F. de Castro, T.M. Kilts, M.F. Young, Matrix Biol. 2014, 35, 223.

[156] J. J. Warren, D. Blanchette, D. V Dawson, A. Teresa, K. R. Phipps, D. Starr, D. R. Drake, Matrix Biol. 2016, 141.

[157] D. Parisuthiman, Y. Mochida, W. R. Duarte, M. Yamauchi, J. Bone Miner. Res. 2005, 20, 1878 .

[158] T. Tashima, S. Nagatoishi, H. Sagara, S. I. Ohnuma, K. Tsumoto, Biochem. Biophys. Res. Commun. 2015, 463, 292.

[159] S. Kalamajski, A. Aspberg, K. Lindblom, D. Heinegård, Å. Oldberg, Biochem. J. 2009, 423, 53.

[160] S. Houari, T. Wurtz, D. Ferbus, D. Chateau, A. Dessombz, A. Berdal, S. Babajko, J. Bone Miner. Res. 2014, 29, 1446.

[161] S. Kalamajski, A. Oldberg, Matrix Biol. 2010, 29, 248.

[162] E. M. Rosset, A. D. Bradshaw, Matrix Biol. 2016, 52-54, 78.

[163] T. Iline-Vul, I. Matlahov, J. Grinblat, K. Keinan-Adamsky, G. Goobes, Biomacromolecules 2015, 16, 2656.

[164] K. Iba, Y. Abe, T. Chikenji, K. Kanaya, H. Chiba, K. Sasaki, T. Dohke, T. Wada, T. Yamashita, J. Bone Miner. Metab. 2013, 31, 399.

[165] A. I. Alford, A. Z. Golicz, A. L. Cathey, A. B. Reddy, Connect. Tissue Res. 2014, 54, 275.

[166] P. Gehron Robey, in Principles of Bone Biology, Vol. 1 (Eds: J.P. Bilezikian, L.G. Raisz, T.J. Martin), Academic Press, Massachusetts, USA 2008, Ch. 17.

[167] G. K. Hunter, Calcif. Tissue Int. 2013, 93, 348.

[168] G. S. Baht, G. K. Hunter, H. A. Goldberg, Matrix Biol. 2008, 27, 600.

[169] W. Bouleftour, L. Juignet, G. Bouet, R. N. Granito, A. Vanden-Bossche, N. Laroche, J. E. Aubin, M. H. Lafage-Proust, L. Vico, L. Malaval, Matrix Biol. 2016, 52-54, 60.

[170] A. A. Joshi, A. J. Chaudhari, C. Li, J. Dutta, S. R. Cherry, D. W. Shattuck, A. W. Toga, R. M. Leahy, Nat. Genet. 2006, 38, 1310.

[171] A. Nampei, J. Hashimoto, K. Hayashida, H. Tsuboi, K. Shi, I. Tsuji, H. Miyashita, T. Yamada, N. Matsukawa, M. Matsumoto, S. Morimoto, T. Ogihara, T. Ochi, H. Yoshikawa, J. Bone Miner. Metab. 2004, 22, 176.

[172] P. S. N. Rowe, Crit. Rev. Oran Biol. Med. 2004, 15, 264.

[173] M. L. Zoch, T. L. Clemens, R. C. Riddle, Bone 2016, 82, 42.

[174] J. P. Gorski, A. Wang, D. Lovitch, D. Law, K. Powell, R. J. Midura, J. Biol. Chem. 2004, $279,25455$.

[175] R. J. Midura, A. Wang, D. Lovitch, D. Law, K. Powell, J. P. Gorski, J. Biol. Chem. 2004, 279, 25464.

[176] N. T. Huffman, J. A. Keightley, C. Chaoying, R. J. Midura, D. Lovitch, P. A. Veno, S. L. Dallas, J. P. Gorski, J. Biol. Chem. 2007, 282, 26002. 
[177] L. J. Schurgers, J. Uitto, C. P. Reutelingsperger, Trends Mol. Med. 2013, 19, 217.

[178] M. L. Cancela, V. Laizé, N. Conceição, Arch. Biochem. Biophys. 2014, 561, 56.

[179] Y. T. Tsao, Y. J. Huang, H. H. Wu, Y. A. Liu, Y. S. Liu, O. K. Lee, Int. J. Mol. Sci. 2017, 18, DOI 10.3390/ijms18010159.

[180] A. Neve, A. Corrado, F. P. Cantatore, J. Cell. Physiol. 2013, 228, 1149.

[181] E. Mavropoulos, A. M. Costa, L. T. Costa, C. A. Achete, A. Mello, J. M. Granjeiro, A. M. Rossi, Colloids Surfaces B Biointerfaces 2011, 83, 1.

[182] L. Brylka, W. Jahnen-Dechent, Calcif. Tissue Int. 2013, 93, 355. 


\section{Chapter 3}

\section{Human osteoclast formation and resorptive function on biomineralized collagen}

Daniel de Melo Pereira, Noel Davison, Pamela Habibović

Published in Bioactive Materials, 2021, 10.1016/j.bioactmat.2021.06.036 


\section{Abstract}

Biomineralized collagen composite materials pose an intriguing alternative to current synthetic bone graft substitutes by offering a biomimetic composition that closely resembles native bone. We hypothesize that this composite can undergo cellular resorption and remodeling similar to natural bone. We investigate the formation and activity of human osteoclasts cultured on biomineralized collagen and pure collagen membranes in comparison to cortical bone slices. Human monocytes/macrophages from peripheral blood differentiate into multinucleated, tartrate-resistant alkaline phosphatase (TRAP)-positive osteoclast-like cells on all substrates. These cells form clear actin rings on cortical bone, but not on biomineralized collagen or pure collagen membranes. Osteoclasts form resorption pits in cortical bone, resulting in higher calcium ion concentration in cell culture medium; however, osteoclast resorption of biomineralized collagen and collagen membranes does not measurably occur. Activity of osteoclast enzymes - TRAP, carbonic anhydrase II (CA-II), and cathepsin-K (CTS-K) - is similar on all substrates, despite phenotypic differences in actin ring formation and resorption. The mesh-like structure, relatively low stiffness, and lack of RGD-containing binding domains are likely the factors responsible for preventing formation of stable actin rings on and resorption of (biomineralized) collagen membranes. This insight helps to guide further research toward the optimized design of biomineralized collagen composites as a more biomimetic bone-graft substitute.

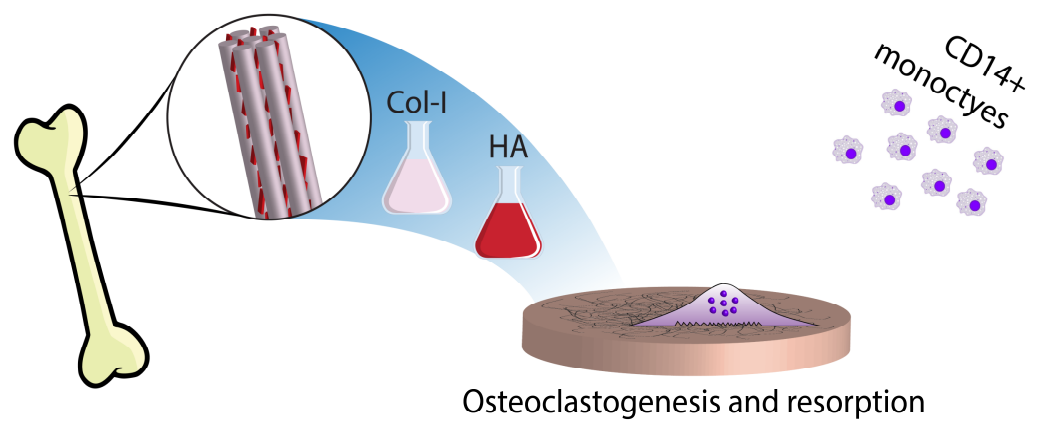




\section{Introduction}

Biomineralization is a process used by many organisms to integrate a mineral entity in an organic setting, with often fascinating results. ${ }^{[1]}$ As the biomineralization process unfolds, there is tight spatial and temporal control over the availability of ions and mineral precursors, and over the way they organize with organic components. This allows for the creation of complex structures, in which function is directly linked to form. Excellent examples for this can be found in the human body, where our bones, teeth, and inner ear are biomineralized tissues with unique properties and function. ${ }^{[2]}$ One of the key functions of human skeleton is to provide mechanical support and protection to soft tissues and organs, for which exceptional mechanical properties are a prerequisite. Bone possesses a unique combination of stiffness and fracture toughness that cannot be solely explained by its composition. In fact, the structural arrangement between soft (organic) and hard (inorganic) components is critical to its performance. ${ }^{[3,4]}$ Biomineralized tissues and the processes by which they form are therefore a natural source of inspiration for the development of new and improved biomaterials.

Many currently available synthetic substitutes of natural bone grafts (auto-, allo- and xenografts) are comprised of collagen, various types of calcium phosphates (CaP), or combinations thereof, inspired by the composition of natural bone tissue.[5,6] Although such biomaterials (partially) mimic the composition of bone, their structure normally does not resemble the intricate organic-inorganic architecture of bone at the submicron scale. As a result, their mechanical properties typically fall short of what is clinically required, especially in load-bearing applications. Moreover, the microenvironment in which the cells relevant to bone formation and remodeling reside is different from their natural microenvironment.

These two drawbacks of current synthetic bone grafts are addressed in biomineralized collagen, consisting of collagen with intrafibrillar $\mathrm{CaP}$ mineral, a material that can be prepared in the laboratory, owing to advances in understanding bone biomineralization processes. ${ }^{[7,8]}$ It is therefore important to further expand our knowledge of biomimetic materials based on biomineralized collagen, and use this knowledge to develop improved bone graft substitutes.

Intrafibrillarly mineralized collagen is commonly prepared by a method named polymer-induced liquid precursor (PILP) method, in which an amorphous calcium 
phosphate (ACP) phase, stabilized by charged polymers (e.g., poly-L-aspartic acid or poly-L-glutamic acid), ${ }^{[9]}$ is able to migrate to the inner confined spaces of collagenI fibers, where it crystallizes into nano-sized hydroxyapatite (HA) platelets, resulting in intrafibrillary mineralized fibers. ${ }^{[10-12]}$ Biomimetic materials based on intrafibrillarly-mineralized collagen replicate key aspects of bone composition and structure to a degree that was not possible before, and are therefore interesting to investigate in the context of bone regeneration. Of note, at the individual fibril level, biomineralized collagen has mineral density similar to bone, as well as similar organization of its main organic and inorganic components, with apatite platelets arranged with their c-axis quasi-parallel to the longitudinal direction of the collagen fiber. The parallel organization of stiffer elongated apatite particles inside the softer collagen matrix phase is likely to contribute to the mechanical properties exhibited by bone at a larger scale. ${ }^{[13,14]}$ Therefore, this mineralized fibril motif constitutes, essentially, the building block of bone's extracellular matrix (ECM), being therefore a promising candidate for building larger scaffolds/constructs that can eventually combine bone-like mechanical properties and bioactivity.

One of the key properties of graft substitute materials for regeneration of large bone defects is their rate of degradation and replacement by native tissue. CaP-based materials, commonly used as synthetic bone graft substitutes, when implanted in a bone environment undergo degradation by both passive physicochemical processes such as hydrolytic dissolution and (mechanical) fragmentation, and by cellmediated processes such as phagocytosis by macrophages and resorption by osteoclasts.[15] Cell-mediated processes are particularly interesting from the perspective of designing materials that are capable of responding to and interacting with the implant environment. Resorption of old ECM by osteoclasts and deposition of new bone tissue by osteoblasts are the two continuous processes responsible for maintaining bone homeostasis. Coordination between these two tightly synchronized processes is ensured by crosstalk between osteoclasts and osteoblasts in a process called bone coupling. ${ }^{[16]}$ When living bone tissue is used as a bone graft material, the graft is slowly resorbed and gradually replaced by new bone, beginning from the edges of the defect toward the middle. This process is defined as creeping substitution of the graft and importantly results in little mechanical weakening of the graft prior to complete bone repair. ${ }^{[17]}$ From this perspective, a synthetic graft material that is recognized and resorbed by resident osteoclasts may 
elicit similar crosstalk and osteogenic coupling factors, ${ }^{[18]}$ thereby retaining its mechanical properties as it is selectively substituted by new bone.

Toward the aim of incorporating bone graft substitutes into the bone remodeling system, it is important to investigate whether biomineralized collagen can undergo resorption by osteoclasts, as this is a first step for cell-mediated synchronous removal of the bone graft substitute and replacement by native tissue. If osteoclastmediated creeping substitution of a biomimetic bone graft substitute could be achieved, it would potentially avoid the challenges in engineering responsive physicochemical degradation rates to match dynamically variable bone formation.

To our knowledge, so far, no studies have directly evaluated osteoclast formation and function on synthetic biomineralized collagen matrices, i.e., collagen matrices with intrafibrillar mineral, although other collagen-hydroxyapatite scaffolds have been investigated in this context. ${ }^{[19,20]}$ Indirect approaches have however been used. Mouse osteoclasts were shown to resorb demineralized bone that was remineralized by the PILP method, with osteopontin as process-directing agent instead of a polymer [21]. Mouse mesenchymal stromal cells (MSCs), cultured in a trans-well system in the presence of collagen scaffolds containing intrafibrillar apatite and silica, were shown to have an inhibitory effect on osteoclastogenesis and resorption when subsequently co-cultured with mouse macrophage cell line RAW 264.7, showcasing the type of cell-to-cell communication that can be affected by these biomaterials. ${ }^{[22]}$

To expand the current knowledge on cell-mediated degradation of biomineralized collagen, we differentiated human monocytes into osteoclasts on mineralized collagen substrates produced with the PILP method and evaluated osteoclast formation and function. For comparison, collagen matrices without mineral, as well as cortical bone slices, a well-known control surface for in vitro osteoclast resorption studies, were used. 


\section{Materials and methods}

\subsection{Materials}

PureCol® collagen type-I solution (col-I, $3 \mathrm{mg} / \mathrm{mL}$, 97\% bovine dermal type I atelocollagen) was purchased from Advanced BioMatrix (San Diego, California, USA, cat\#5005). Poly-L-aspartic acid sodium salt (pAsp, Mw = $27 \mathrm{kDa}$ ) was purchased from Alamanda Polymers (Huntsville, Alabama, USA, cat\#000-D200). 1-Ethyl-3-[3dimethylaminopropyl] carbodiimide hydrochloride (EDC), Nhydroxysulfosuccinimide (sulfo-NHS), calcium chloride dihydrate, potassium phosphate dibasic, and all other chemicals were ordered from Sigma-Aldrich (St. Louis, Missouri, USA). Recombinant human M-CSF and soluble RANK-L cytokines were from Peprotech (Connecticut, USA, cat\#3002510 and cat\#3100110).

\subsection{Preparation of collagen membranes}

In brief, collagen gels with a concentration of $2 \mathrm{mg} / \mathrm{mL}$ were prepared by mixing $1412 \mu \mathrm{L}$ of collagen solution, $352 \mu \mathrm{L}$ of $10 x$ phosphate buffered saline (PBS), and 236 $\mu \mathrm{L}$ of $0.1 \mathrm{M} \mathrm{NaOH}$. Gels with a volume of $2 \mathrm{~mL}$ were formed in modified $5 \mathrm{~mL}$ syringes (Sigma Aldrich, cat\#Z248010), at $37{ }^{\circ} \mathrm{C}$ for 24 hours. The syringes containing the gel were inverted on top of nylon meshes with a pore size of $40 \mu \mathrm{m}$ (Fischer Scientific, cat \#11587522) and left for $24 \mathrm{~h}$ at $37^{\circ} \mathrm{C}$ to allow the gels to loose water under their own weight. After this compression step, syringes were removed, and the formed collagen membranes remained in the cell strainer for another $24 \mathrm{~h}$, before being washed with PBS and cross-linked with $50 \mathrm{mM}$ EDC, $25 \mathrm{mM}$ sulfo-NHS in 50 $\mathrm{mM}$ MES buffer $(\mathrm{pH}=7.0)$, overnight at room temperature. The procedure for making collagen membranes is illustrated in Supplementary Figure 1. The following day, the membranes were washed with PBS, incubated with $0.1 \mathrm{Na}_{2} \mathrm{HPO}_{4}$ and $2 \mathrm{M}$ $\mathrm{NaCl}$ for $2 \mathrm{~h}$, to quench the remaining activated carboxylic acid residues, and finally washed three times with PBS for $2 \mathrm{~h}$. The membranes were kept in PBS at $4{ }^{\circ} \mathrm{C}$ for up to one week before being used for cell culture or characterization. All steps were performed in a sterile environment.

\subsection{Mineralization of collagen membranes}

Mineralization solution was prepared according to the PILP method ${ }^{[8]}$. Briefly, precursor solutions of calcium $\left(9 \mathrm{mM} \mathrm{CaCl}_{2}\right)$ and phosphate $\left(4.2 \mathrm{mM} \mathrm{K}_{2} \mathrm{HPO}_{4}\right)$ were 
prepared in a buffer with $50 \mathrm{mM}$ TRIS base and $150 \mathrm{NaCl}$ keeping $\mathrm{pH}$ at 7.8-8.0 at room temperature. Prior to incubation, pAsp was added to the calcium precursor solution, mixed and allowed to rest for $5 \mathrm{~min}$, followed by addition of the same volume of phosphate precursor solution. The final concentration of pAsp was 100 $\mu \mathrm{g} / \mathrm{mL}$. After mixing the two precursor solutions, the collagen membranes were added ( $40 \mathrm{~mL}$ of solution was used per membrane) and left at $37^{\circ} \mathrm{C}$ for 7 days. Before cell culture, mineralized membranes were washed with PBS and incubated in cell culture medium for $3-4 \mathrm{~h}$. All steps were performed in a sterile environment.

\subsection{Characterization of collagen and biomineralized collagen membranes}

\subsubsection{Scanning electron microscopy and energy-dispersive X-ray} spectroscopy (SEM-EDS)

For SEM-EDS, collagen and biomineralized collagen membranes were dehydrated in a series of ethanol in PBS (30\%, 40\%,50\%), followed by a series of ethanol in water $(60 \%, 70 \%, 80 \%, 90 \%, 100 \%), 15$ min per step and finally in hexadimethylsiloxane for $30 \mathrm{~min}$ before air drying overnight. Samples were glued with silver paint (Ted Pella, USA, cat\#16062) onto aluminum stubs and sputter-coated with a $2 \mathrm{~nm}$ layer of iridium for SEM imaging, or left without coating for elemental analysis. Imaging was done at $2-10 \mathrm{kV}$ at a working distance of $2-10 \mathrm{~mm}$ with a Teneo microscope (FEI, USA) using the T1 or ETD detectors in Optiplan mode. Elemental analysis was done by EDS (Team EDS from EDAX, USA) on a VERSA electron microscope (FEI, USA).

2.4.2. Transmission electron microscopy and selected area electron diffraction (TEM-SAED)

For TEM-SAED, collagen fibrils were formed on a gold grid (Electron Microscope Sciences, USA, cat\#FCF400-Au) by placing the grid on a $50 \mathrm{uL}$ droplet of col-I solution ( $2 \mathrm{mg} / \mathrm{mL}$ as described above before the compression step) and incubating at $37^{\circ} \mathrm{C}$ in a humid environment. After washing with distilled water, the collagencoated grid was placed on top of a $50 \mathrm{uL}$ droplet of mineralization solution (prepared as described above) and incubated for 1 or 7 days at $37^{\circ} \mathrm{C}$. Finally the grids were washed with distilled water and air-dried at room temperature overnight. Samples were imaged at $120 \mathrm{kV}$ (Tecnai G2 Spirit, FEI, OR, United Stated). For calculating the d-spacings from electron diffraction, a new gold grid was used to obtain the 
diffraction pattern of gold which was compared with known d-spacings, from which the reciprocal space scale was calculated.

\subsubsection{Thermogravimetric analysis (TGA)}

For TGA, collagen and biomineralized collagen membranes were washed with MilliQ water to remove salts, blotted with filter paper and stored in microcentrifuge tubes. Samples were burned in air, up to $800^{\circ} \mathrm{C}$, at a rate of $5^{\circ} \mathrm{C} / \mathrm{min}$, using a Q500 TGA (TA Instruments, Belgium). The leftover weight at $700{ }^{\circ} \mathrm{C}$ was considered to be the mineral content of the membrane.[23]

2.5. Monocyte/macrophage culture and differentiation into osteoclasts Poietics $^{\text {tm }}$ human peripheral blood CD14+ monocytes were obtained from Lonza (Basel, Switzerland, cat\#2W-400B, lot\#647890). Upon thawing the cryovial, cells were transferred to a $15 \mathrm{~mL}$ tube and $9 \mathrm{~mL}$ warm basic cell culture medium $(\alpha$-MEM (Lonza, cat\#BE02-002F) supplemented with 10\% (v/v) HyClone ${ }^{\text {tm }}$ FetalClone $^{\text {tm }}$ serum (Thermo Fisher Scientific, USA, cat\#10780245, lot\#HXSH3008003), 100 U/ml penicillin, $100 \mu \mathrm{g} / \mathrm{ml}$ streptomycin and $20 \mathrm{ng} / \mathrm{mL}$ M-CSF) was added, followed by centrifugation at $300 \mathrm{rcf}$ for $5 \mathrm{~min}$. Cells were re-suspended in basic cell culture medium, counted, and seeded at a density of 500.000 cells $/ \mathrm{cm}^{2}$ into 96 -well or 48 well plates containing the various substrates. For the first 3 days of culture, the basic medium was modified to contain $35 \mathrm{ng} / \mathrm{mL}$ M-CSF. Then, the culture continued in either basic (20 ng/mL M-CSF) or in differentiation medium (basic medium supplemented with $40 \mathrm{ng} / \mathrm{mL}$ RANK-L) for up to 21 days, counting from the addition of RANK-L, with medium refreshment every 2-3 days.

2.6. Immunohistochemical analysis of osteoclasts by confocal laser microscopy After 14 or 21 days of culture, cells on different substrates were washed once with warm PBS, and fixed with warm 10\% formaldehyde in PBS for $15 \mathrm{~min}$ at room temperature. Cells were washed three times with PBS, and permeabilized with $0.2 \%$ (v/v) Tween-20 in PBS for $10 \mathrm{~min}$ at room temperature. ELF-97 phosphate substrate (Thermo Fisher Scientific, USA, cat\#E6601) was used for visualization of activity of tartrate-resistant alkaline phosphatase (TRAP) according to the protocol published by Filgueira. ${ }^{24]}$ Briefly, 20x concentrated ELF-97 substrate was diluted in a buffer composed of $110 \mathrm{mM}$ acetate $(\mathrm{pH}=5.2), 1.1 \mathrm{mM}$ sodium nitrite and $7.4 \mathrm{mM}$ tartrate. 
Cells were incubated with diluted ELF-97 for $15 \mathrm{~min}$, washed three times with PBS, incubated with 1:200 PBS-diluted AlexaFluor ${ }^{\circledR} 488$ phalloidin (Thermo Fisher Scientific)) for $20 \mathrm{~min}$, washed three times with PBS and finally incubated with 1 $\mu \mathrm{g} / \mathrm{mL}$ DAPI (Thermo Fisher Scientific, USA, cat\#1306) in PBS for $10 \mathrm{~min}$. All incubation steps were at room temperature, in the dark. Imaging $(n=2)$ was done with a SP8 STED confocal laser microscope (Leica Microsystems, Germany) using 25x and 86x water-immersion objectives. Sequential scanning was performed with two detector configurations, one for DAPI and ELF-97, and another for phalloidin 488. This allowed observation of ELF-97 and phalloidin AF-488 without any signal interference, despite the considerable overlap of the emission wavelength of both fluorophores. A $405 \mathrm{~nm}$ excitation laser was used for DAPI and ELF-97, with detection wavelengths of 417-478 nm for DAPI and 570-621 nm for ELF-97. A 488 $\mathrm{nm}$ excitation laser was used for phalloidin AF-488, with detection wavelengths of 499-570 $\mathrm{nm}$. Large, multinucleated $(\mathrm{n}>2)$ and TRAP-positive cells were considered to be osteoclasts. Quantification of TRAP activity ${ }^{1}$ was performed by image analysis using Image J (version 1.52r). The number of TRAP positive pixels was counted for each osteoclast, and expressed as fraction of total cell area. Details of this analysis are provided in a short video as a part of the available dataset published with this study.

\subsection{Identification and quantification of resorption sites}

\subsubsection{Optical profilometry}

After 21 days, cells were removed from the substrates by incubation in $1 \%$ Triton X100 in PBS for $30 \mathrm{~min}$ in an ultrasonic waterbath with ice. The substrates were washed with distilled water and air-dried before analysis. Bone slices, collagen and biomineralized collagen membranes $(n=3)$ were imaged with a VKX-200 3D laser microscope (Keyence, Japan) using the 20x objective. All substrates were imaged completely by stitching. A reference surface $(z=0)$ was defined for individual images using a planar tilt correction (bone slice) or a waveform removal with cutoff

\footnotetext{
${ }^{1}$ Here it should read semi-quantification, i.e. the comparison of amounts of fluorescent substrate (ELF-97) converted by TRAP between different conditions. The type of image analysis presented in this chapter is not sufficient to determine the activity of the TRAP enzyme. We ask the reader to keep this in mind while analyzing Figures 2 and 3 as well as their corresponding discussion. The original text was kept as published in Bioactive Materials and this note was added for clarification.
} 
set at $200 \mu \mathrm{m}$ ((biomineralized) collagen membranes). A threshold below the reference surface was set for bone slices $(z=-2 \mu \mathrm{m})$ and for both biomimetic membranes $(z=-5 \mu \mathrm{m})$, and all data points below this threshold were considered a result of resorption. Quantification was done using the volumetric tool.

\subsubsection{SEM}

After 21 days, the cells were either fixed on or removed from the substrates, as described above. The substrates with cells were dehydrated in a series of ethanol in PBS (30\%, 40\%, 50\%), followed by a series of ethanol in water $(60 \%, 70 \%, 80 \%, 90 \%$, $100 \%), 15$ min per step and finally in hexadimethylsiloxane for $30 \mathrm{~min}$ before air drying overnight. Samples were glued onto aluminium stubs with carbon tape and silver paint, and sputter-coated with a $2 \mathrm{~nm}$ layer of iridium. Imaging was done in a TENEO electron microscope, using the T1 detector in Optiplan mode, with the beam at $2-10 \mathrm{kV}$ and working distance of $2-10 \mathrm{~mm}$.

\subsubsection{Ion-coupled plasma mass spectrometry (ICP-MS)}

At each medium refreshment, a $100 \mu \mathrm{L}$-sample of medium was collected and kept at $-30{ }^{\circ} \mathrm{C}$ until analysis. After collecting samples at the different time points, they were thawed, and $50 \mu \mathrm{L}$ of cell culture medium was diluted in $950 \mu \mathrm{L}$ measuring matrix (1\% $\mathrm{HNO}_{3}$ with $20 \mathrm{ppb}$ scandium). A standard curve of calcium and phosphorous was prepared, ranging from 100 to $4000 \mathrm{ppb}$. Scandium was used as internal standard to compensate signal drift from the instrument and other factors. For each type of substrate analyzed (bone slice and biomineralized collagen), there were two conditions (basic medium and differentiation medium) each with 3 replicates, i.e., medium collected from 3 different wells of a well plate. Since collagen membranes without mineral did not contain any calcium, the ICP-MS analysis for this condition was not performed.

\subsection{Enzymatic activity assays}

At 7,14 , or 21 days, the cell culture medium was removed and substrates were washed once with warm PBS. PBS was removed before freezing the plates containing samples at $-80{ }^{\circ} \mathrm{C}$. When the samples at different time points were collected, the plates were thawed, the substrates were transferred into new plates, and $200 \mu \mathrm{L}$ lysis buffer (1\% Triton X-100 in PBS) was added to each well. Two more 
freeze-thaw cycles were performed, followed by a $30 \mathrm{~min}$ incubation in an ultrasonic waterbath with ice. Cell lysates were then transferred to $0.5 \mathrm{~mL}$ microcentrifuge tubes and either analyzed immediately, or frozen for later analysis. All handling of cell lysate samples was performed in ice to delay protein degradation.

2.8.1. Quantification of tartrate-resistant alkaline phosphatase (TRAP) activity

For quantification of TRAP activity $(\mathrm{n}=3), 20 \mu \mathrm{L}$ of cell lysate was mixed with 130 $\mu \mathrm{L}$ of buffer containing $10 \mathrm{mM}$ 4-nitrophenylphosphate, $0.1 \mathrm{M}$ sodium acetate $(\mathrm{pH}$ 5.8), $0.15 \mathrm{M} \mathrm{KCl}, 0.1 \%$ Triton $\mathrm{X}-100,10 \mathrm{mM}$ sodium tartrate, $1 \mathrm{mM}$ ascorbic acid, and $0.1 \mathrm{mM} \mathrm{FeCl6}$. The mixture was incubated at $37^{\circ} \mathrm{C}$ for $1 \mathrm{~h}$, quenched with $100 \mu \mathrm{L} 0.3$ $\mathrm{M} \mathrm{NaOH}$, and finally the absorption was measured at $405 \mathrm{~nm}$. A standard curve of 4-nitrophenol was used to convert absorption values into moles of liberated phosphate.

\subsubsection{Carbonic Anhydrase II (CA-II) activity}

Quantification of CA-II activity $(\mathrm{n}=3)$ was done by proxy via its known esterase activity. In brief, $50 \mu \mathrm{L}$ of cell lysate was added to $50 \mathrm{uL}$ of buffer, containing 12.5 $\mathrm{mM}$ Tris ( $\mathrm{pH}=7.5), 75 \mathrm{mM} \mathrm{NaCl}$ and $2 \mathrm{mM}$ 4-nitrophenylacetate. Absorbance was measured after $5 \mathrm{~min}$ at $405 \mathrm{~nm}$ with a CLARIOstar Plus microplate reader (BGM Labtech, Germany). A standard curve of 4-nitrophenol was used to convert absorption values into moles of liberated acetate.

\subsubsection{Quantification of cathepsin-K (CTS-K) activity}

Quantification of CTS-K activity $(\mathrm{n}=3$ ) was performed as described before.[25] Briefly, $10 \mu \mathrm{L}$ of cell lysate was further diluted in $40 \mu \mathrm{L}$ of PBS, and then mixed with $50 \mu \mathrm{L}$ buffer containing $0.1 \mathrm{M}$ sodium acetate, $4 \mathrm{mM}$ EDTA, $4 \mathrm{mM}$ DTT $(\mathrm{pH}=5.5)$ and $100 \mu \mathrm{M}$ Z-LR-AMC. The plate was incubated at $37^{\circ} \mathrm{C}$ for $30 \mathrm{~min}$, and fluorescence was measured at an excitation/emission wavelength of $365 / 440 \mathrm{~nm}$. A standard curve of aminomethylcoumarin (AMC) was used to convert the fluorescence intensity into moles of liberated AMC.

2.9. Statistical analysis

Statistical analysis was performed in GraphPad Prism (version 8.3). The Student's ttest was used to compare resorption data from optical profilometry between cells 
cultured in basic and differentiation medium for each substrate, and two-way ANOVA was used to compare $\mathrm{Ca}^{2+}$ data from ICP-MS as well as enzymatic activity data from the respective assays. Differences in means between conditions were tested at each timepoint. A Bonferroni post-hoc test was used to correct for multiple comparisons (one family for all comparisons). Mean differences were considered statistically significant for $\mathrm{p}$-value $<0.05$. In all figures, the following notation applies: ${ }^{*} \mathrm{p}<0.05 ;{ }^{* *} \mathrm{p}<0.001 ;{ }^{* * *} \mathrm{p}<0.0001$.

\section{Results and discussion}

In this study, we aimed to investigate the behavior of human osteoclasts on synthetic biomineralized collagen in comparison to pure collagen and bovine bone slices. Understanding osteoclast formation, function, and related cell-mediated degradation of biomaterials is important for developing biomimetic bone graft substitutes based on biomineralized collagen.

\subsection{Biomineralized membranes mimic composition and structure of bone at submicron scale}

Bovine collagen membranes were successfully produced and used as a base material in this study. The membranes had a porous, mesh-like structure, composed of randomly oriented collagen fibers, as shown by SEM (Figure 1A). After mineralization using the PILP method, no nodular mineral deposits were observed on the collagen fiber surface, as is the case with conventional CaP coating methods, such as incubation in simulated body fluid (SBF).[26] Fiber diameter increased from $81 \pm 23$ to $185 \pm 38(n=20)$ after the mineralization process and elemental analysis by EDS showed presence of calcium and phosphorus (Figure 1B) which was not the case when pure collagen was analyzed (Figure 1A). An EDS line scan over the cross section of a biomineralized membrane detected calcium and phosphorus across the entire thickness of the membrane (about $100 \mu \mathrm{m}$ ) and not only on the surface, indicating that the polymer-ACP complexes were able to diffuse through the membrane and mineralize it completely (Figure 1C). TGA indicated that the inorganic mass fraction (intrafibrillar CaP mineral) was about $78 \%$, while collagen and residual water mass fraction made up the remaining $22 \%$ that were burned away after heating to $800{ }^{\circ} \mathrm{C}$ (Figure 1D). These results provided convincing 
evidence that $\mathrm{CaP}$ mineral was present in the biomineralized membrane, in amounts similar to that of secondary bone, consisting of $\approx 65 \mathrm{wt} . \%$ mineral phase, $\approx 25 \mathrm{wt} . \%$ organic (of which around $90 \%$ is collagen type I), and $\approx 10 \mathrm{wt} . \%$ water, ${ }^{[7]}$ and that its distribution throughout the membrane was homogeneous. Moreover, as mineral was not observed on the surface of collagen fibers, it likely deposited within the fibers as intrafibrillar mineral. Further confirmation was obtained by TEM, where, after incubation of collagen fibers for 1 day in the PILP mineralization solution, nano-sized crystal particles were observed in close association with collagen fibers, as dark streaks parallel to each other and aligned with the collagen fiber direction (Figure 1E). Evidence of the identity of these mineral deposits was obtained after 7 days of mineralization by SAED of a fully mineralized collagen fiber, which showed diffraction arcs with d-spacings and angles corresponding to reflections from HA crystal planes 002, 004, 112, 211 and 300 (Figure 1F-G). ${ }^{[7]}$ The 002 and 004 planes are normal to the c-axis of the hexagonal crystal cell, and the narrow angle of their arcs in the diffraction pattern corresponds to the angular dispersion of the c-axis direction of HA crystal platelets, showing that the c-axis is aligned with the direction of the collagen fiber.

These results show that $\mathrm{CaP}$ mineral was present in the form of nano-sized HA crystals embedded in the collagen fibers, making this biomimetic material similar to bone in composition and structure, at the length scale of nano- to micrometer. ${ }^{[8]}$

3.2. Multinucleated osteoclast-like cells are able to form on collagen, biomineralized collagen as well as on cortical bone slices

Upon optimization of culture protocols, it was possible to differentiate human peripheral blood-derived monocytes into osteoclast-like cells when cultured on the biomineralized collagen membranes, as well as on collagen membranes without mineral and cortical bone slices. Large, multinucleated, and TRAP-positive cells were observed on all three surfaces after 14 days of culture in differentiation medium (Figure 2A, 2C, 2E). On cortical bone slices, F-actin structures having high fluorescence intensity were observed in the form of ring and crescent shapes with punctate morphology (Figure 2A). These structures were different from other actin structures which were also observed, such as stress fibers. The high intensity punctate structures represent podosomal belts and actin rings, which define the sealing zone and resorption lacunae. ${ }^{[27]}$ 

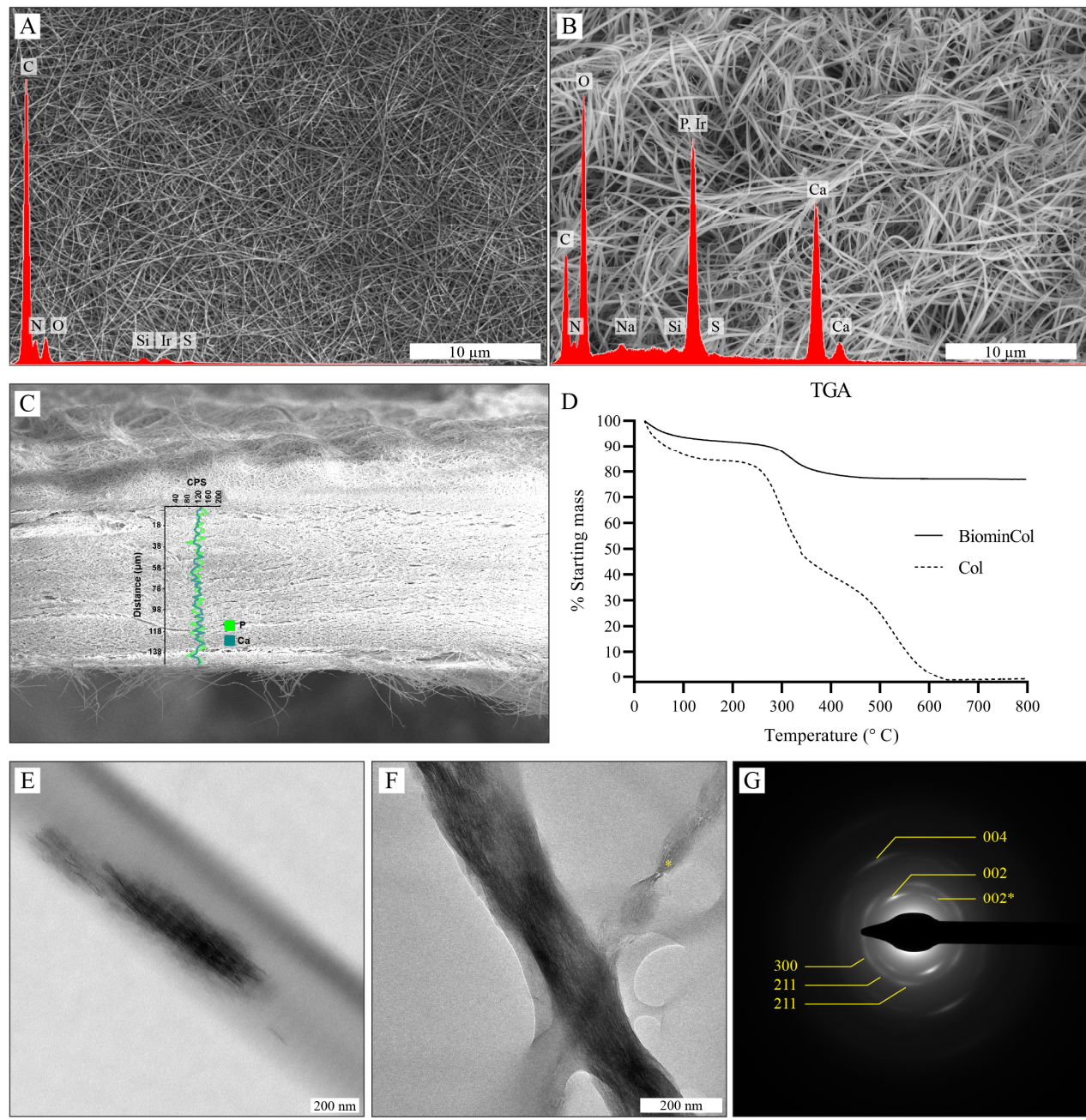

Figure 1. Characterization of collagen and biomineralized collagen membranes. SEM and corresponding EDS of a collagen membrane (A) and biomineralized collagen membrane (B). Cross-section SEM micrograph of a biomineralized collagen membrane, and EDS line scan analysis over its thickness (calcium in blue, phosphorus in green) (C). Quantification of mineral content by TGA of collagen (Col) and biomineralized collagen (BiominCol) (D). TEM micrographs of collagen fibrils after 1 day $(\mathrm{E})$ and 7 days $(\mathrm{F})$ of mineralization using the PILP method. SAED of the area imaged in $(\mathrm{F})$, with the diffraction arcs corresponding to hydroxyapatite reflections highlighted $(\mathrm{G})$. The $002^{*}$ reflection corresponds to the fibril marked with * in $(\mathrm{F})$. 
Actin rings provide evidence that osteoclasts are functional, able to seal off a compartment of the underlying material for resorption. Actin structures with high fluorescence intensity, likely podosomes, were also observed in osteoclasts in close contact with both collagen- and biomineralized collagen membranes, but they lacked the organization seen on cortical bone slices, not having a clear dotted pattern or a well-defined ring- or crescent shape (Figure 2C, 2E).

Similar observations were made after 21 days of culture (Figure 2B, 2D, 2F). Actin rings were visible on cortical bone slices, indicating that osteoclasts were still active at this time point (Figure 2B). Actin structures observed on the ventral membrane of cells on collagen membranes with and without intrafibrillar mineral did not display the structural organization of podosome belts or actin rings, even after 21 days (Figure 2D, 2F). Quantification of TRAP activity by image analysis after 14 and 21 days of culture showed similar levels of activity per osteoclast on cortical bone slices, biomineralized collagen and collagen without mineral (Figure 3).

These results suggest that, while osteoclast-like cells have the ability to form on collagen and biomineralized collagen surfaces, their function is likely to be impaired, given that the typical structures associated with functional osteoclasts were not observed after 14 or 21 days of culture in differentiation medium. This seems to be a direct effect of the properties of these biomimetic membranes, as mature, resorbing osteoclasts were able to form on the cortical bone slices under the same conditions. It is generally recognized that both the physical and chemical properties of the substrate have an effect on osteoclast formation, surface adhesion and normal function, i.e., resorptive activity. Presence of integrin-binding motifs, ${ }^{[28,29]}$ substrate stiffness, ${ }^{[30,31]}$ porosity, ${ }^{[32]}$ and topographical roughness, ${ }^{[27]}$ were all shown to affect actin ring formation or resorptive activity.

Of particular interest to this study are the effects of integrin-binding motifs and physical properties of the biomineralized collagen membranes and collagen membranes without mineral, as these are the two major properties that distinguish these biomimetic materials from cortical bone. Cell-recognized, integrin-binding RGD sequences are present in various proteins of the ECM of cortical bone, [33] but not in (biomineralized) collagen membranes.[34,35] The RGD motif is important for osteoclast adhesion and activation because it is the ligand to integrin $\alpha_{\mathrm{v}} \beta_{3}$, also known as vitronectin receptor, the most highly expressed integrin in osteoclasts, for which there is ample evidence that, in its absence, no resorption takes place. ${ }^{[36,37]}$ 
However, even in the absence of RGD, osteoclasts have shown the ability to form actin rings on collagen-based surfaces, given the right physical properties. In the study by Nakamura et al., it was shown that osteoclasts were able to form actin rings when cultured on collagen-coated plastic coverslips, but not when residing in a collagen gel.[29] Importantly, the collagen-coated substrates were likely stiffer, flatter and less porous than the collagen gels, which were softer and presented a fibrillary, mesh-like surface topography. These differences plausibly impacted osteoclast function. Based on these findings, the authors suggested that actin ring formation depends not only on RGD binding but also on the physical properties of the substrate such as stiffness and surface topography. In comparison, the mesh-like fibrillary structure of the collagen membranes used in the present study was more similar to the collagen gel than to the collagen-coated coverslips described by Nakamura et al., in terms of surface structure and mechanical properties, ${ }^{[38]}$ which may explain why no resorption lacunae were observed on these surfaces. While it is difficult to determine precisely which material property was most consequential for this cellular effect, Touaitahuata et al. (2013) showed that osteoclasts formed actin rings on softer materials like polyacrylamide gels and polydimethylsiloxane, with stiffness ranging from 30 to around $1800 \mathrm{kPa}$, suggesting that the porous surface structure rather than stiffness may be the dominant causal factor. ${ }^{[31]}$ Porosity, and particularly pore size, is known to impact osteoclast function, as shown by Davison et al. in a study with $\beta$-TCP ceramic discs with differently sized surface microstructures (grains and pores).

Figure 2. (next page) Osteoclasts on cortical bone, biomineralized collagen and collagen surfaces after 14 or 21 days of culture in differentiation medium. F-actin (red), nuclei (blue) and TRAP (green). In cortical bone (A,B), bright actin staining shows organized actin ring structures, crescent shaped (pointed in A by *) or circular (traced line in A), are visible within the cell. Orthogonal projections of $\mathrm{xz}$ (bottom) and $\mathrm{yz}$ (right side) show that these structures (arrowheads) are enclosing a basal recess oriented deeper into the substrate surface than the adjacent substrate surface, suggesting these are sealing zones undergoing active resorption. Arrows in the orthogonal projections indicate the vertical direction. Actin rings are still visible in cortical bone after 21 days (traced line in B). Brightly stained punctate actin structures $\left(^{*}\right)$ are also seen in some osteoclasts on biomineralized collagen $(C, D)$, resembling podosomes but without the ring-like organization seen in cortical bone. Side view shows that these structures (arrowheads) are likely in contact with the surface of the material, but they do not as clearly enclose a contiguous zone as observed in cortical bone. Similar structures appeared less often and not as concentrated on collagen surfaces (E,F). All scale bars are $50 \mu \mathrm{m}$. 
14 days
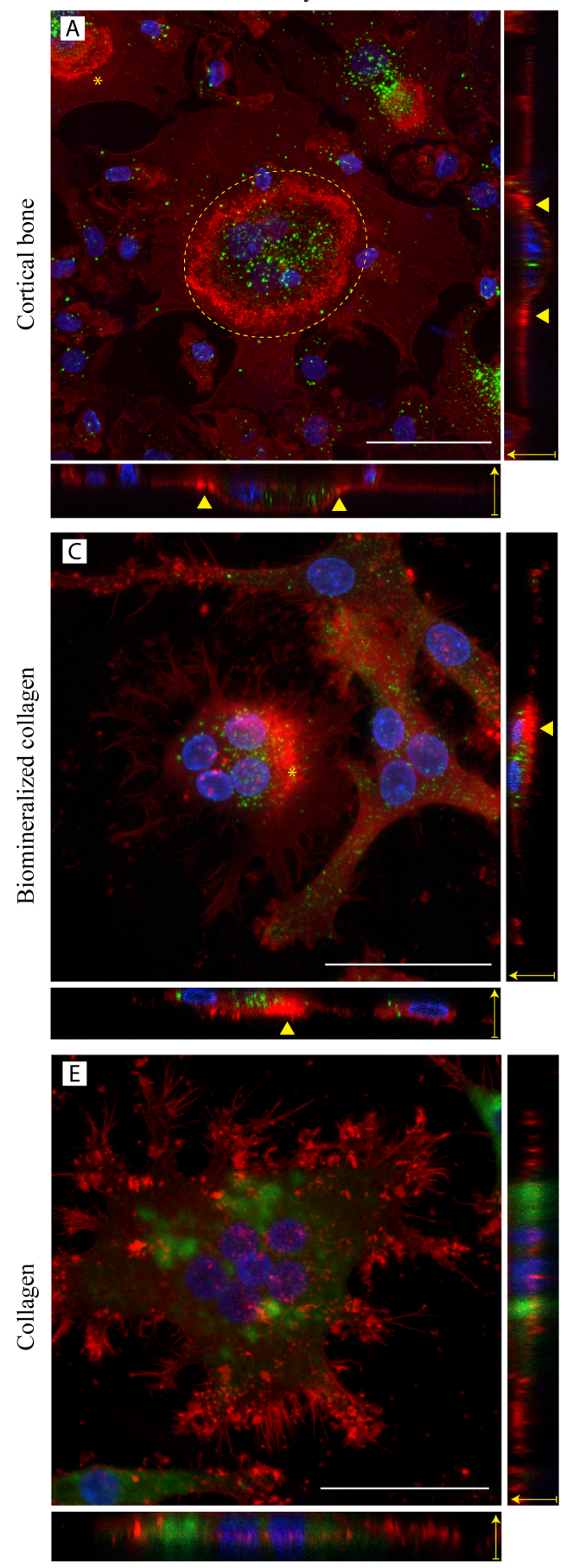

21 days
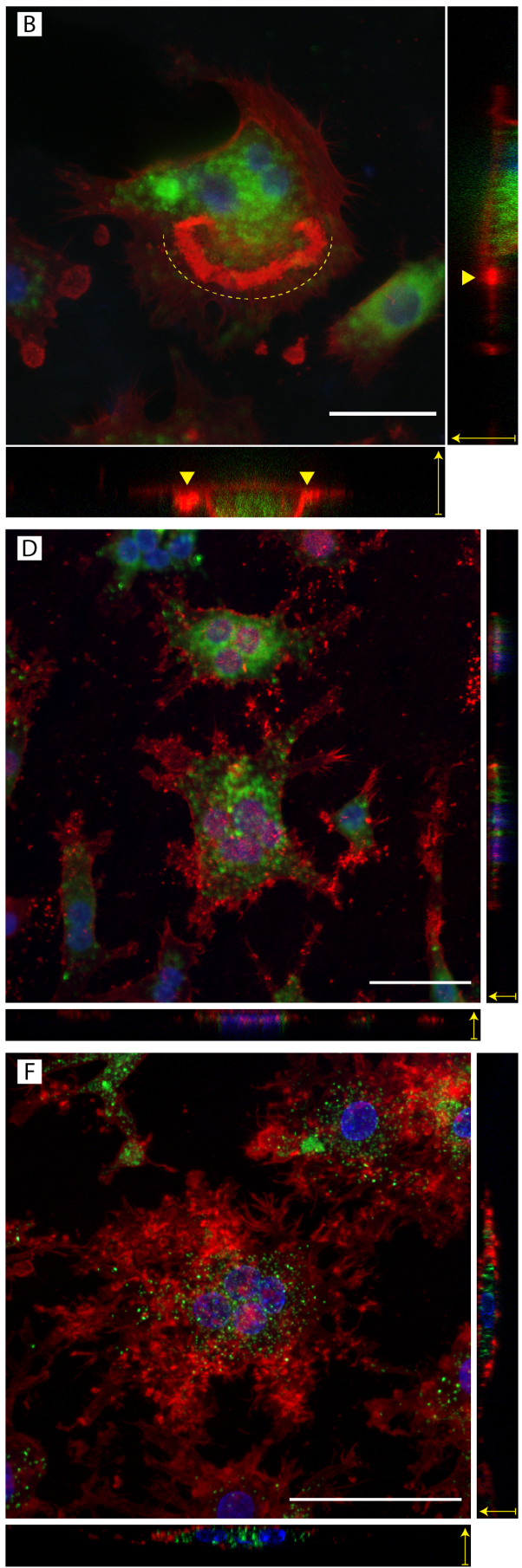
No actin rings were observed for osteoclasts on $\beta$-TCP surfaces with micron-sized structural features (grain diameter $3.7 \mu \mathrm{m}$ and pore size $1.8 \mu \mathrm{m}$ ), and there was also no resorption, but on $\beta$-TCP surfaces with submicron features (grain diameter 0.9 $\mu \mathrm{m}$ and pore size $0.6 \mu \mathrm{m}$ ) osteoclasts showed clear actin rings and there was ample resorption. This is in line with findings about the formation and stability of sealing zones on titanium surfaces of varying roughness, where an inverse correlation was found between the stability and translocation rate of sealing zones and roughness increasing from $\mathrm{R}_{\mathrm{a}}=1$ to $4.5 \mu \mathrm{m}$. ${ }^{[27]}$

This data suggests an interplay between stiffness, pore size and surface structure, which in our study was such that it inhibited actin ring formation and resorption.

A

TRAP - Bone slices

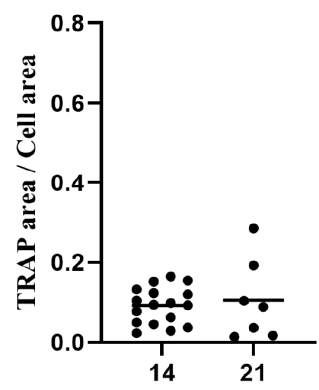

Time (days)
B

TRAP - BiominCol

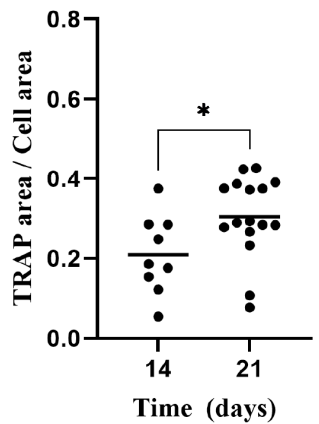

C

TRAP - Collagen

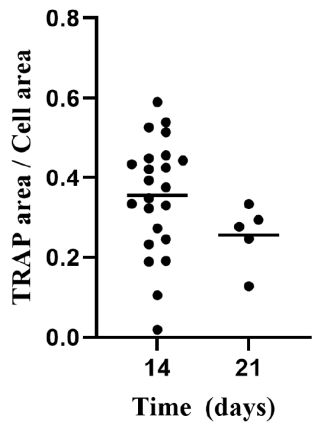

Figure 3. Semi-quantitative comparison of TRAP activity between osteoclasts cultured for 14 or 21 days on cortical bone slices, biomineralized collagen and collagen membranes. TRAP activity is shown as the area fraction of TRAP-positive pixels relative to the whole cell area, for each osteoclast (each point represents one osteoclast).

3.3. Osteoclasts are able to resorb cortical bone but they do not resorb collagen and biomineralized collagen membranes

Following an osteoclast culture of 21 days in differentiation medium, SEM analysis of the different surfaces was performed. Abundant resorption pits were observed on cortical bone slices (Figure 4A, 4D). In contrast, no evidence was found for resorption on either biomineralized collagen or collagen membranes without mineral (Figure 4B, 4E, 4C, 4F). Occasionally, the fibrillar structure of the membrane surface in close contact with osteoclasts appeared to be disturbed and disorganized 
compared to the surrounding cell-free surface, but it is unclear whether this is an effect of resorption or other cell-mediated effects, such as tension exerted on the fibers or enzymatic activity.

Images of the surface made using an optical profilometer and the corresponding image quantification corroborated that extensive resorption occurred on cortical bone slices (Figure 5A, 5D, 5G). Bone slices on which cells were cultured in differentiation medium showed a mean resorbed volume that was about 25 times higher than when cells were cultured in basic medium, without RANK-L (Figure 5D). Similarly, the resorbed area, expressed as a percentage of total area, was $1 \%$ and $19 \%$ when cells were cultured in basic and differentiation medium, respectively (Figure 5G). Both difference in resorbed volume and area were statistically significant, with $\mathrm{p}<0.0001$.
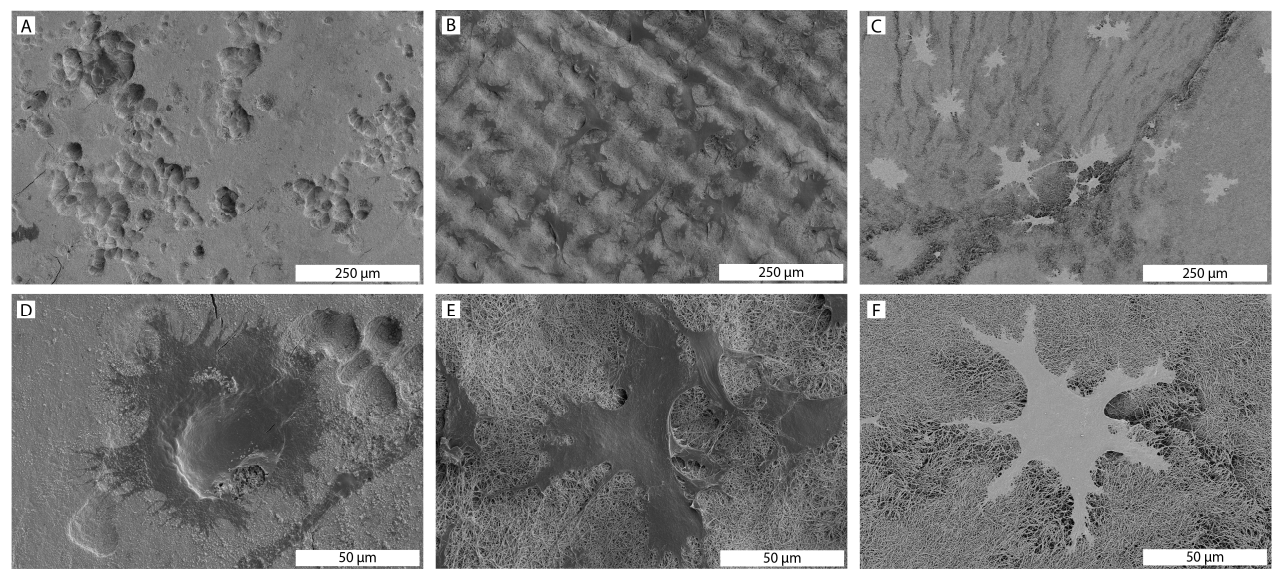

Figure 4. SEM images of osteoclasts on cortical bone (A,D), biomineralized collagen (B,E) and collagen $(\mathrm{C}, \mathrm{F})$ surfaces after 21 days in culture. Resorption lacunae can be seen in cortical bone, but not on biomineralized collagen or on collagen membranes.

While optical profilometry is a useful tool for analyzing the extent of resorption on bone slices, which are relatively smooth, this type of analysis is more difficult to perform on rough and uneven surfaces, because the algorithm is not able to differentiate between native topographical features (e.g., collagen fibers) and resorbed pits or trenches. 

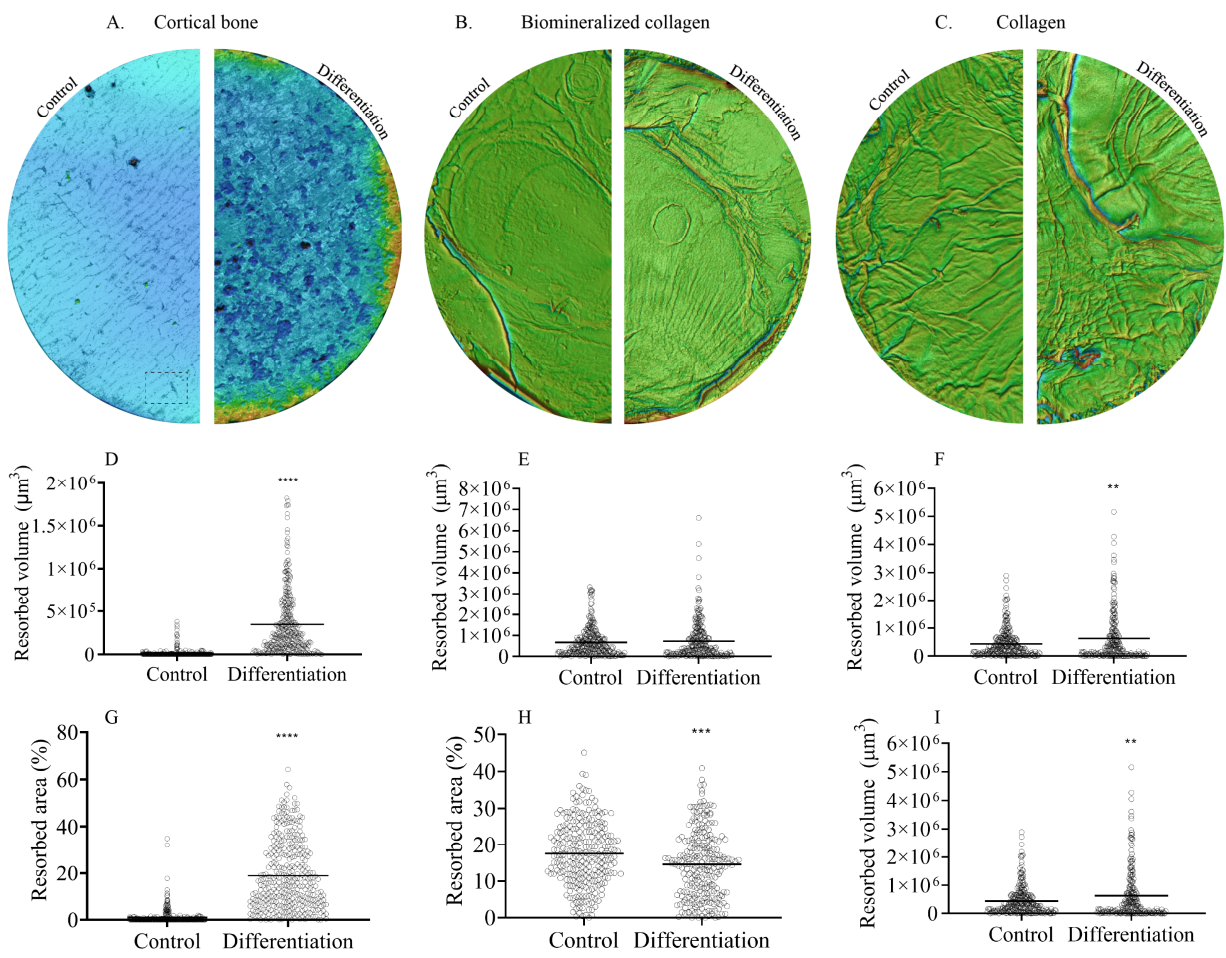

Figure 5. Volume and area resorbed by osteoclasts cultured on cortical bone, biomineralized collagen and collagen surfaces. The control condition were cells cultured in basic medium, with $20 \mathrm{ng} / \mathrm{mL}$ M-CSF but without RANK-L, whereas the differentiation medium contained $20 \mathrm{ng} / \mathrm{mL}$ M-CSF and $40 \mathrm{ng} / \mathrm{mL}$ RANK-L. Each data point represents a field-of-view as captured and analyzed by optical profilometry (dashed rectangle). Differences in resorbed volume and area between control and differentiation conditions are significant for cortical bone $(\mathrm{p}<0.0001)$. Difference in resorbed area is significant for biomineralized collagen $(\mathrm{p}<$ 0.001). Difference in resorbed volume is significant for collagen $(p=0.0034)$.

As a result, more noise is present in the data, obscuring small differences in potential resorption; because of this, only very extensive resorption activity such as what was seen on cortical bone is robustly detectable. With this limitation of the technique in mind, the results showed that no significant difference in the mean resorbed volume was observed between cells cultured on biomineralized collagen membranes in basic or differentiation medium (Figure 5E). A difference of $3 \%(p<0.001)$ was observed for the resorbed area, with a larger area for cells cultured in basic medium, which is plausibly an artifact of the technique used (Figure $5 \mathrm{H}$ ). Similar to the results 
obtained for mineralized collagen membranes, cells cultured on collagen without mineral in differentiation medium showed a slightly higher mean resorbed volume (1.4 times $(p=0.0034)$ ) than the control (Figure 5F), while no significant differences were found in the percentage resorbed area (Figure 5I). Notably, the percentage "resorbed area" in both basic and differentiation medium for both pure collagen and mineralized collagen was almost as high as the value observed for cells cultured on bone slices in the differentiation medium, which clearly shows that on these porous surfaces, the technique cannot distinguish between the structural properties of the material itself and structural changes resulting from resorption.

To make the technique suitable for analysis of rough surfaces, it is important to have a proper definition of a reference plane or level $(z=0)$, from which any concavity (with $\mathrm{z}<0$ ), would be counted as resulting from cell-induced resorption. The reference value for a relatively flat surface with low roughness $\left(R_{a}<1 \mu \mathrm{m}\right)$, such as the bone slice, is easy to define with good accuracy, for example by definition of a reference plane. A surface presenting surface topography such as wrinkles or complex shapes in the tens or hundreds of micrometers, requires a more complex method for defining a good reference, for example using a band-pass filter that removes (i.e., makes $\mathrm{z}=0$ ) any feature larger than a pre-defined cutoff wavelength. The trade off in choosing the cutoff dimension involves over-estimation of resorption (cutoff dimension too large) or under-estimation of resorption (cutoff dimension too small), which translates as more noise across all conditions, as noted above.

To provide further evidence for resorption by osteoclasts, we measured the concentration of $\mathrm{Ca}^{2+}$ in cell culture medium over a 21-day experiment by using ICPMS. The results showed that for cells cultured on cortical bone slices in differentiation medium, a higher $\mathrm{Ca}^{2+}$ concentration was observed between day 7 and day 21 in comparison with cells cultured in basic medium (Figure 6A), with differences being statistically significant between day 9 and day 21. These results confirmed that there was ample resorption of cortical bone slices by the osteoclasts cultured on them over a period of 21 days. According to the profile of the $\mathrm{Ca}^{2+}$ concentration, it is suggested that the onset of osteoclast resorption occurred between day 4 and day 7, further increased up to day 16 and then leveled off until the end of the experiment at 21 days. It should be noted that the only difference between basic and differentiation medium is that in the latter, RANK-L was present. 
This means that effects such as $\mathrm{CaP}$ precipitation from cell culture medium (previously observed for CaP-containing materials ${ }^{[39]}$ ), or dissolution of the intrafibrillar mineral, are expected to occur in both basic and differentiation medium, and thus should not affect the differences between the two conditions.

In contrast to bone slices, the analysis of the cell culture medium of cells cultured on biomineralized collagen membrane in basic or differentiation medium showed no significant differences in $\mathrm{Ca}^{2+}$ concentration at any time point (Figure 6B), suggesting that indeed, no measurable osteoclastic resorption occurred on this substrate, which is in line with other results.
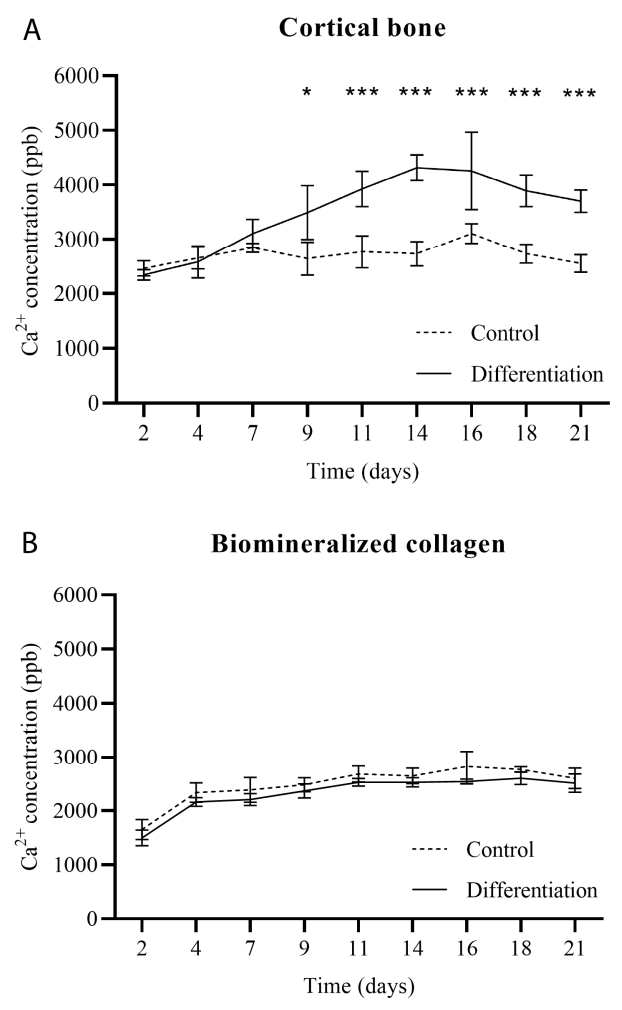

Figure 6. Concentration of calcium ions in cell culture medium, measured by ICP-MS at each moment of media change, for a total of 21 days. In the control condition cells were cultured in basic medium containing $20 \mathrm{ng} / \mathrm{mL} \mathrm{M-}$ CSF, but without RANK-L, and in the differentiation condition cells were cultured in medium containing 20 $\mathrm{ng} / \mathrm{mL} \mathrm{M}-\mathrm{CSF}$ and $40 \mathrm{ng} / \mathrm{mL}$ RANK-L. On cortical bone slices, differences between control and differentiation are visible from day 7 becoming significant from day 9 onwards. On biomineralized collagen surfaces, there are no appreciable differences in the calcium ion concentration between the control and differentiation conditions, indicating that no substantial (if any) resorption occurred. 


\subsection{Enzymatic activity of TRAP, CTS-K and CA-II does not necessarily correlate with osteoclast resorptive activity}

To further characterize the osteoclasts that formed on different surfaces, and investigate to which extent the enzymatic activity of cells is predictive of osteoclast formation and resorptive activity, we performed enzyme activity assays on key osteoclast markers TRAP, CTS-K and CA-II after 7, 14 and 21 days of culture in basic or differentiation medium.

During bone resorption, TRAP concentration in serum has been shown to increase, although its exact role in bone resorption is still not fully understood. ${ }^{[40]}$ As is shown in Figure 7A, at 7 days, the TRAP activity of cells cultured on bone slices was comparable between culture in basic and differentiation medium. Between 7 and 14 days, an increase in TRAP activity was observed in both basic and differentiation medium, however, this increase was more enhanced when cells were cultured in the differentiation medium. This resulted in higher activity in the differentiation medium than in the basic medium, although this difference was not statistically significant. Between 14 and 21 days, TRAP activity showed a further slight increase in basic medium, whereas a decrease was observed in differentiation medium, which resulted in comparable TRAP activity for the two conditions of cells cultured on bone slices for 21 days. The observed decrease in TRAP enzymatic activity between 14 and 21 days was in contrast with the immunofluorescence results (Figure 7A, 2B), where TRAP activity nodules were still abundant after 21 days (Figure 3A). The ICP-MS results (Figure 6A) suggested that osteoclasts were actively resorbing up to day 21, although a decreasing trend in $\mathrm{Ca}^{2+}$ content of the medium between 14 and 21 days of culture was observed, which is suggestive of levelling off of resorptive activity and in line with a decrease in TRAP activity observed here.

For cells cultured on biomineralized collagen and collagen without mineral (Figure $7 \mathrm{~B}, 7 \mathrm{C})$, there was an increase in TRAP activity between 7 and 21 days of culture in differentiation medium, whereas TRAP activity levels remained fairly constant when the culture was performed in basic medium. As a result, significantly higher TRAP activity values were measured in the differentiation medium after 14 and 21 days on both substrates, and they were comparable between collagen with and without mineral. 

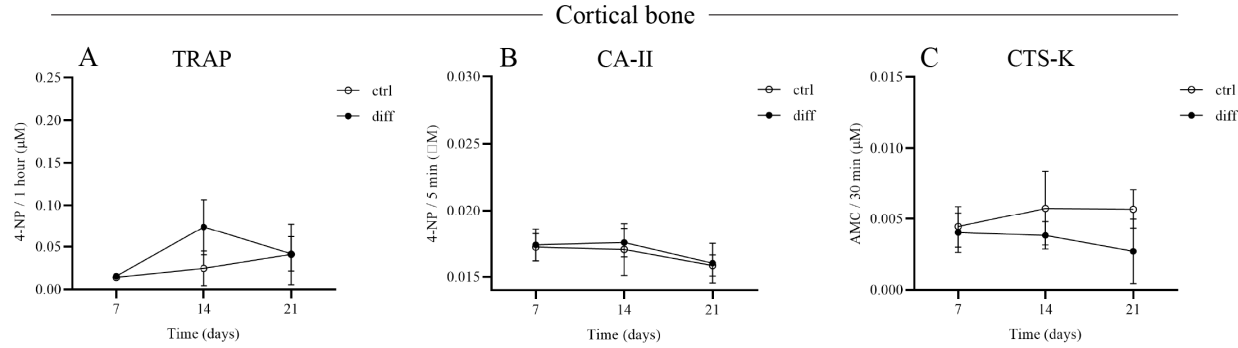

Biomineralized collagen
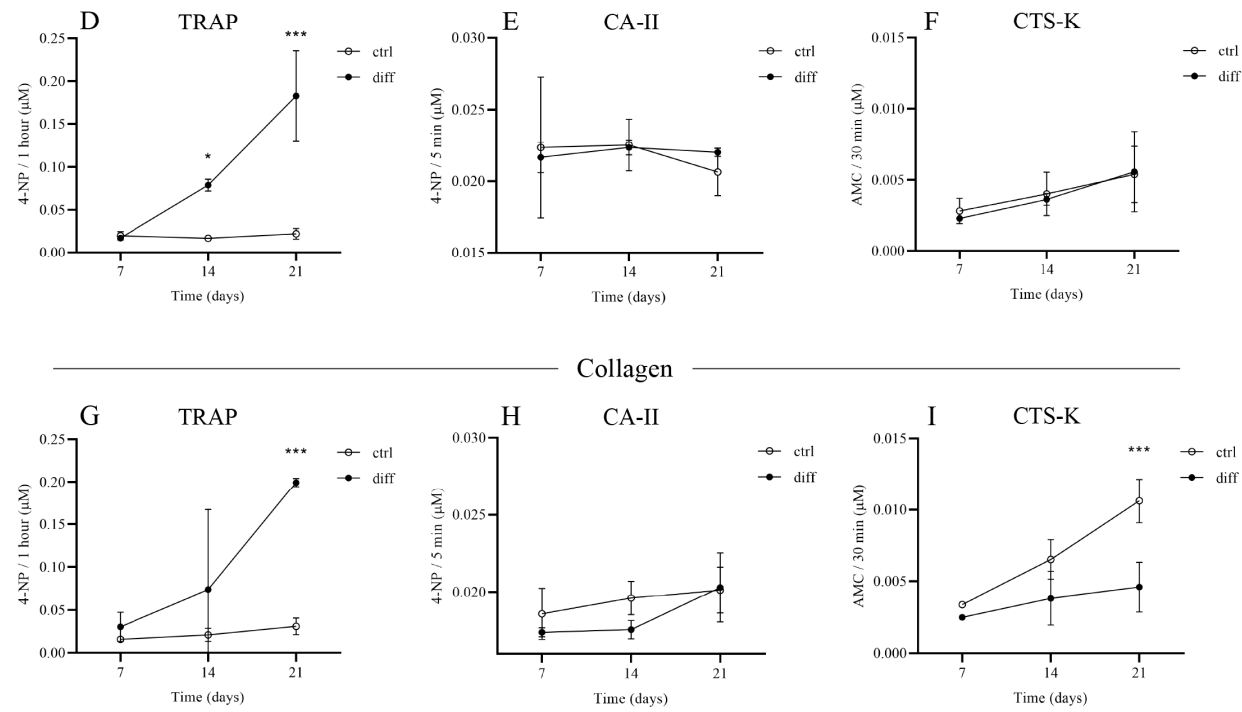

Figure 7. Activity of enzymes TRAP, CA-II and CTS-K, measured in lysates of osteoclasts cultured on cortical bone, biomineralized collagen, and collagen for 7, 14, and 21 days. TRAP activity was measured as the moles of 4-nitrophenyl converted during $1 \mathrm{~h}$ incubation with 4nitrophenylphosphate. CA-II activity was measured as the moles of 4-nitrophenyl converted during 5 min incubation with 4-nitrophenylacetate. CTS-K activity was measured as the moles of aminomethylcoumarin converted during $30 \mathrm{~min}$ incubation with Z-LR-AMC.

These results confirmed the fluorescence data showing osteoclast formation and TRAP activity up to 21 days of culture in the differentiation medium on both substrates (Figure 3B, 3C), although there was no proof of osteoclastic resorption. There is ample evidence that TRAP activity correlates well with osteoclastogenesis, with higher TRAP activity values indicating more osteoclasts, irrespective of the underlying substrate. ${ }^{[41-43]}$ This evidence appears to extend to biomineralized 
collagen and collagen without mineral as well, as shown by the results of our study. However, correlation with resorption is less clear-cut, as it seems to depend on the substrate, but also on whether the enzyme is secreted or intracellular. ${ }^{[25]}$

CA-II is an intracellular enzyme that acts as a proton source for acidification of the resorption lacunae. ${ }^{[4]}$ Here, CA-II activity showed a different trend from TRAP activity on all surfaces. In the case of cortical bone slices (Figure 7D), CA-II activity remained at the similar level between 7 and 14 days and decreased between 14 and 21 days for both cells cultured in basic and in differentiation medium. No differences were observed between the two media, at any time point. Similarly, for cells cultured on biomineralized collagen (Figure 7E), no differences were observed between basic and differentiation medium at any of the time points. Moreover, no temporal changes were observed for either condition. Finally, on collagen matrices without mineral (Figure 7F), in contrast to cortical bone and biomineralized collagen, a small increase in CA-II activity was observed between 7 and 21 days for both cells cultured in basic and in differentiation medium. At days 7 and 14, surprisingly, a higher CAII activity was measured in basic than in differentiation medium. The results of CAII activity do not seem to correlate with the TRAP activity results or the osteoclastogenesis observed by fluorescence immunohistochemistry. Such a correlation has been reported in literature. ${ }^{[45]}$, albeit on calcium phosphate cements, which osteoclasts were also shown to resorb. Our findings are in agreement with earlier work that the CA-II activity does not directly correlate with osteoclastic resorption. [25].

Regarding the activity of CTS-K, a secreted protease responsible for type I collagen degradation during bone resorption [46], for cells cultured on cortical bone slices (Figure 7G), no obvious temporal changes were observed, and a somewhat higher, though statistically not significant, CTS-K activity was found for cells cultured in basic than in differentiation medium after 14 and 21 days, which does not corroborate the pronounced resorptive activity in the differentiation medium as observed by immunofluorescence, SEM and ICP-MS. This might be because we measured enzyme activity in cell lysates, as opposed to extracellular, secreted CTS$\mathrm{K}$, which has previously been shown to correlate with resorbed area when cells were cultured on dentin slices [25]. On biomineralized collagen (Figure 7H) and collagen without mineral (Figure 7I), an increasing trend in CTS-K activity was observed between 7 and 21 days. While no differences were observed between cells cultured 
in basic and differentiation medium on biomineralized collagen, on collagen without mineral, higher CTS-K levels were measured in basic than in differentiation medium.

Taken together, these results indicate that intracellular enzymatic activity was not a reliable predictor of osteoclast resorption, as there was little correlation among TRAP, CA-II and CTS-K activity in cell lysates. Moreover, the activity of these enzymes did not reflect the evidence for osteoclast formation and resorptive activity as obtained using immunohistochemistry and morphological investigation using SEM. Other factors, such as location of enzyme (intra- versus extracellular), composition of the biomaterial, and $\mathrm{pH}$ of the culture medium may have all played a role in these results. For quantification of resorption, ICP-MS analysis of $\mathrm{Ca}^{2+}$ concentration in cell culture medium proved to be a more useful indicator of resorptive activity on calcium-containing biomaterials.

The results presented here show that while osteoclast-like cell formation seems to readily occur on biomineralized collagen, substrate resorption does not. No clearly defined actin rings were observed on biomineralized or pure collagen, and no resorption of these materials was detected. We hypothesize that the two main factors contributing to this are i) the porous, mesh-like structure of the membranes; and ii) the lack of ligands to the vitronectin receptor. The large pores of the mesh-like fibrillary collagen are likely to have prevented the normal formation of actin rings, a structure that is key to sealing off a section of material for degradation, and without which no resorption can take place. Moreover, the lack of ligands to the vitronectin receptor may have further contributed to this effect.

Despite its biomimetic design, biomineralized collagen, as prepared, was not resorbed like cortical bone. Although the biomineralized collagen showed closer-tonative properties than other materials to date, it is clear that, as far as osteoclast function is concerned, a key aspect of the native tissue was not replicated sufficiently. We suggest that a more dense substrate, with pore size reduced to the submicron scale, would likely support osteoclast resorption. Therefore the next steps in developing biomineralized collagen materials should focus on densification of the matrix, with either closer packing of the collagen fibers, or with extrafibrillar mineral to reduce porosity. Moreover, functionalizing the material with appropriate ligands to the vitronectin receptor is expected to enhance its ability to be resorbed. 


\section{Conclusion}

We investigated osteoclast formation and activity on biomineralized collagen and collagen membranes and made a comparison with cortical bone, a material that is well known for supporting normal osteoclast resorption. We found that biomineralized collagen and collagen membranes support osteoclast-like cell formation via RANK-L induced fusion of monocyte/macrophage precursors. Osteoclasts expressed TRAP on all materials, but only formed clear actin rings on cortical bone, which was also the only material osteoclasts were able to resorb. We hypothesized that the fibrillar, porous structure of the collagen membrane's surface, together with the lack of RGD ligands to the vitronectin receptor, prevented the formation of a stable sealing zone. Toward the development of more biomimetic biomineralized collagen composites, future studies should focus on the role of material porosity and fibrillary structure in directing osteoclast actin ring formation and resorption. Progress in these areas may result in better bone graft substitutes that can be more readily remodeled by osteoclasts and substituted by new bone tissue. 


\section{Data availability statement}

The raw data generated for this study are publicly available at https://doi.org/10.34894/UAL6GN.

\section{Acknowledgements}

The authors would like to thank Carmen López-Iglesias, Hans Duimel, and Raimond Ravelli from M4I Institute - Division of Nanoscopy (Maastricht University) for the assistance and guidance provided with TEM-SAED analyses. Myraise van Stijn and Enabling Technologies/DSM are acknowledged for assistance with SEMEDS analyses.

\section{Author Contributions}

DP designed and performed the experiments. ND provided critical input during experimental design. DP, ND and $\mathrm{PH}$ wrote the manuscript. $\mathrm{PH}$ supervised the project and reviewed the manuscript. All authors contributed to the article and approved the submitted version.

\section{Funding}

This research has been made possible with the support of the Dutch Province of Limburg (LINK project). PH gratefully acknowledges the Gravitation Program 'Materials-Driven Regeneration', funded by the Netherlands Organisation for Scientific Research (NWO).

\section{Conflict of interest}

The authors confirm that there are no known conflicts of interest associated with this publication and there has been no significant financial support for this work that could have influenced its outcome. 


\section{Supplementary Material}

A

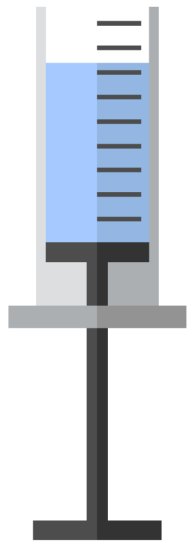

B

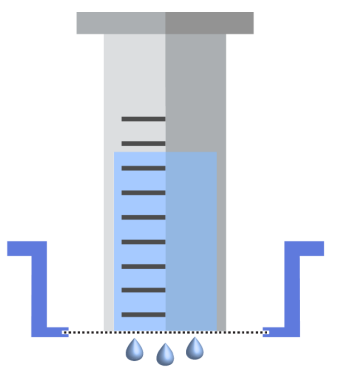

C

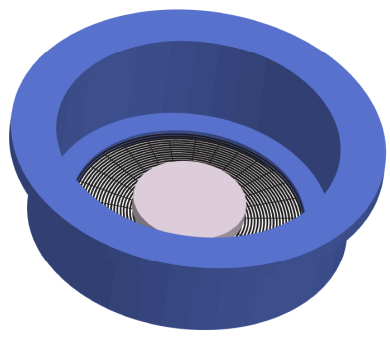

Supplementary Figure 1. Method for preparation of collagen membranes. Gel formation in upright modified syringe for $24 \mathrm{~h}$ (A); piston removal and syringe body placement on a cell strainer with a nylon mesh of $40 \mu \mathrm{m}$ pore size for $24 \mathrm{~h}$ (B); removal of syringe body, flattened collagen membrane remains on the cell strainer for another $24 \mathrm{~h}(\mathrm{C})$. 


\section{References}

[1] H. A. Lowenstam, S. Weiner, On Biomineralization, Oxford University Press, 1989.

[2] Yunxia Wang Lundberg, Y. Xu, K. D. Thiessen, K. L. Kramer, Dev. Dyn. 2016, 176, 139.

[3] U. G. K. Wegst, H. Bai, E. Saiz, A. P. Tomsia, R. O. Ritchie, Nat. Mater. 2015, 14, 23.

[4] O. A. Tertuliano, J. R. Greer, Nat. Mater. 2016, 15, 1195.

[5] T. Kurien, R. G. Pearson, B. E. Scammell, Bone Jt. J. 2013, 95 B, 583.

[6] P. Baldwin, D. J. Li, D. A. Auston, H. S. Mir, R. S. Yoon, K. J. Koval, J. Orthop. Trauma 2019, 33, 203.

[7] M. J. Olszta, X. Cheng, S. S. Jee, R. Kumar, Y. Y. Kim, M. J. Kaufman, E. P. Douglas, L. B. Gower, Mater. Sci. Eng. R Reports 2007, 58, 77.

[8] Y. Li, T. T. Thula, S. Jee, S. L. Perkins, C. Aparicio, E. P. Douglas, L. B. Gower, Biomacromolecules 2011, 13, 49.

[9] T. T. Thula, F. Svedlund, D. E. Rodriguez, J. Podschun, L. Pendi, L. B. Gower, Polym. Basel 2011, 3, 10.

[10] F. Nudelman, K. Pieterse, A. George, P. H. H. Bomans, H. Friedrich, L. J. Brylka, P. A. J. Hilbers, G. De With, N. A. J. M. Sommerdijk, Nat. Mater. 2010, 9, 1004.

[11] F. Nudelman, P. H. H. Bomans, A. George, G. de With, N. A. J. M. Sommerdijk, Faraday Discuss. 2012, 159, 357.

[12] W. J. E. M. Habraken, J. Tao, L. J. Brylka, H. Friedrich, L. Bertinetti, A. S. Schenk, A. Verch, V. Dmitrovic, P. H. H. Bomans, P. M. Frederik, et al., Nat. Commun. 2013, 4, 1507.

[13] M. Balooch, S. Habelitz, J. H. Kinney, S. J. Marshall, G. W. Marshall, J. Struct. Biol. 2008, 162, 404.

[14] A. K. Nair, A. Gautieri, M. J. Buehler, Biomacromolecules 2014, 15, 2494.

[15] Z. Sheikh, M. N. Abdallah, A. A. Hanafi, S. Misbahuddin, H. Rashid, M. Glogauer, Materials (Basel). 2015, 8, 7913.

[16] S. Takeshita, T. Fumoto, K. Matsuoka, K. Park, H. Aburatani, S. Kato, M. Ito, K. Ikeda, J. Clin. Invest. 2013, 123, 3914.

[17] T. T. Roberts, A. J. Rosenbaum, Organogenesis 2012, 8, 114.

[18] Y. Shiwaku, L. Neff, K. Nagano, K.-I. Takeyama, J. de Bruijn, M. Dard, F. Gori, R. Baron, PLoS One 2015, 10, 1.

[19] H. Domanschke, M. Gelinsky, B. Burmeister, R. Fleig, T. Hanke, A. Reinstorf, W. Pompe, A. Rosen-Wolff, Tissue Eng. 2006, 12, 949.

[20] A. Bernhardt, S. Thieme, H. Domaschke, A. Springer, A. Rösen-Wolff, M. Gelinsky, J. Biomed. Mater. Res. - Part A 2010, 95, 848.

[21] D. E. Rodriguez, T. Thula-Mata, E. J. Toro, Y. W. Yeh, C. Holt, L. S. Holliday, L. B. Gower, Acta Biomater. 2014, 10, 494.

[22] K. Jiao, L. Niu, Q. Li, F. Chen, W. Zhao, J. Li, J. H. Chen, C. W. Cutler, D. H. Pashley, F. R. Tay, Acta Biomater. 2015, 19, 23.

[23] S. Jee, T. T. Thula, L. B. Gower, Acta Biomater. 2010, 6, 3676.

[24] L. Filgueira, J. Histochem. Cytochem. 2004, 52, 411.

[25] A. Bernhardt, K. Koperski, M. Schumacher, M. Gelinsky, Eur. Cells Mater. 2017, 33, 28. 
[26] S. M. Maria, C. Prukner, Z. Sheikh, F. Mueller, J. E. Barralet, S. V. Komarova, J. Biomed. Mater. Res. - Part B Appl. Biomater. 2014, 102, 903.

[27] D. Geblinger, C. Zink, N. D. Spencer, L. Addadi, B. Geiger, J. R. Soc. Interface 2012, 9, 1599.

[28] M. Gano, P. Zigrino, S. Colucci, G. Zambonin, L. Trusolino, M. Serra, N. Baldini, A. Teti, P. C. Marchisio, A. Z. Zallone, Exp. Cell Res. 1994, 212, 209.

[29] I. Nakamura, N. Takahashi, T. Sasaki, E. Jimi, T. Kurokawa, T. Suda, J. Bone Miner. Res. 1996, 11, 1873.

[30] G. Pawlak, V. Vives, A. Blangy, E. Planus, C. Albiges-Rizo, in Mechanosensing Biol. (Ed: M. Noda), Springer Japan, Tokyo, 2011, pp. 193-213.

[31] H. Touaitahuata, E. Planus, C. Albiges-Rizo, A. Blangy, G. Pawlak, Eur. J. Cell Biol. 2013, 92, 139.

[32] N. L. Davison, B. ten Harkel, T. Schoenmaker, X. Luo, H. Yuan, V. Everts, F. Barrèrede Groot, J. D. de Bruijn, Biomaterials 2014, 35, 7441.

[33] J. P. Gorski, K. D. Hankenson, in Princ. Bone Biol. (Fourth Ed. (Eds: J. P. Bilezikian, T. J. Martin, T. L. Clemens, C. J. Rosen), Academic Press, 2020, pp. 359-378.

[34] Y. Takada, X. Ye, S. Simon, Genome Biol. 2007, 8, DOI 10.1186/gb-2007-8-5-215.

[35] A. V Taubenberger, M. A. Woodruff, H. Bai, D. J. Muller, D. W. Hutmacher, Biomaterials 2010, 31, 2827.

[36] K. P. McHugh, K. Hodivala-Dilke, M. H. Zheng, N. Namba, L. Jonathan, D. Novack, X. Feng, F. P. Ross, R. O. Hynes, S. L. Teitelbaum, J. Clin. Invest. 2000, 105, 433.

[37] I. Nakamura, N. Takahashi, E. Jimi, N. Udagawa, T. Suda, Mod. Rheumatol. 2012, 22, 167.

[38] D. D. M. Pereira, M. Schumacher, P. Habibovic, 2021, 1.

[39] A. M. C. Barradas, V. Monticone, M. Hulsman, C. Danoux, H. Fernandes, Z. Tahmasebi Birgani, F. Barrère-De Groot, H. Yuan, M. Reinders, P. Habibovic, et al., Integr. Biol. (United Kingdom) 2013, 5, 920.

[40] J. M. Halleen, S. L. Alatalo, H. Suominen, S. Cheng, A. J. Janckila, H. K. Väänänen, J. Bone Miner. Res. 2000, 15, 1337.

[41] J. P. Rissanen, M. I. Suominen, Z. Peng, J. M. Halleen, Calcif. Tissue Int. 2008, 82, 108.

[42] S. Vahabzadeh, M. Roy, S. Bose, J. Mater. Chem. B 2015, 3, 8973.

[43] N. L. Davison, J. Su, H. Yuan, J. J. J. P. van den Beucken, J. D. de Bruijn, F. B. de Groot, Eur. Cells Mater. 2015, 29, 314.

[44] W. S. Sly, M. P. Whyte, V. Sundaram, R. E. Tashian, D. Hewett-Emmett, P. Guibaud, M. Vainsel, H. J. Baluarte, A. Gruskin, M. Al-Mosawi, N. Engl. J. Med. 1985, 313, 139.

[45] M. Schumacher, A. S. Wagner, J. Kokesch-Himmelreich, A. Bernhardt, M. Rohnke, S. Wenisch, M. Gelinsky, Acta Biomater. 2016, 37, 184.

[46] M. J. Bossard, T. A. Tomaszek, S. K. Thompson, B. Y. Amegadzie, C. R. Hanning, C. Jones, J. T. Kurdyla, D. E. McNulty, F. H. Drake, M. Gowen, et al., J. Biol. Chem. 1996, $271,12517$. 


\section{Chapter 4}

\section{Cobalt-containing calcium phosphate induces resorption of biomineralized collagen by human osteoclasts}

Daniel de Melo Pereira, Matthias Schumacher, Pamela Habibović

Published in Biomaterials Research, 2021, 25:6, 1-13 


\section{Abstract}

Biomineralized collagen, consisting of fibrillar type-I collagen with embedded hydroxyapatite mineral, is a bone-mimicking material with potential application as a bone graft substitute. Despite the chemical and structural similarity with bone extracellular matrix, no evidence exists so far that biomineralized collagen can be resorbed by osteoclasts. The aim of the current study was to induce resorption of biomineralized collagen by osteoclasts by a two-fold modification: increasing the calcium phosphate content and introducing cobalt ions $\left(\mathrm{Co}^{2+}\right)$, which have been previously shown to stimulate resorptive activity of osteoclasts. To this end, we produced biomineralized collagen membranes and coated them with a cobaltcontaining calcium phosphate (CoCaP). Human osteoclasts, derived from CD14+ monocytes from peripheral blood, were differentiated directly on the membranes. Upon fluorescent staining of nuclei, F-actin and tartrate-resistant alkaline phosphatase, the cells were analyzed by laser confocal microscopy. Their resorption capacity was assessed by scanning electron microscopy (SEM), as well as indirectly quantified by measuring the release of calcium ions into cell culture medium. The CoCaP coating increased the mineral content of the membranes by $4 \%(\mathrm{wt})$ and their elastic modulus from 1 to $10 \mathrm{MPa}$. The coated membranes showed a sustained $\mathrm{Co}^{2+}$ release in water of about $7 \mathrm{nM}$ per 2 days. In contrast to uncoated membranes, on CoCaP-coated biomineralized collagen membranes, osteoclasts sporadically formed actin rings, and induced formation of resorption lacunae, as observed by SEM and confirmed by increase in $\mathrm{Ca}^{2+}$ concentration in cell culture medium. The effect of the CoCaP layer on osteoclast function is thought to be mainly caused by the increase of membrane stiffness, although the effect of $\mathrm{Co}^{2+}$, which was released in very low amounts, cannot be fully excluded. This work shows the potential of this relatively simple approach to induce osteoclast resorption of biomineralized collagen, although the extent of osteoclast resorption was limited, and the method needs further optimization. Moreover, the coating method is suitable for incorporating bioactive ions of interest into biomineralized collagen, which is typically not possible using the common biomineralization methods, such as polymer-induced liquid precursor method. 


\section{Introduction}

Biomineralized collagen is a promising material in the context of therapies for repair and regeneration of bone defects. It is composed of fibrillar type-I collagen and intrafibrillar mineral in the form of crystallites of calcium phosphate $(\mathrm{CaP})$ of the apatitic phase, oriented with their c-axis parallel to the collagen fibers. As such, biomineralized collagen mimics the composition and structure of bone, at the submicron scale, with an unprecedented level of detail.[1]

Replicating the structure of bone-like mineralized collagen fibril, and using this unit as a building block for larger constructs, is proposed as a biomimetic method to obtain synthetic bone graft substitutes with load-bearing capacity, as the characteristic structural organization of the collagen and apatite components of the mineralized fibril is one of the major contributors to the mechanical properties of bone ${ }^{[2,3]}$ Biomineralized collagen also presents a closer-to-native microenvironment to cells than current bone graft substitutes, which are typically composed of CaP or bioactive glasses. ${ }^{[4]}$

A desirable aspect of a bone graft substitute, besides biocompatibility, is the ability to interact with the microenvironment upon implantation, resulting in direct contact between implanted biomaterial and surrounding bone, thus avoiding undesired fibrous encapsulation. Moreover, biomaterials for bone regeneration should ideally be incorporated in the cellular process of bone remodeling. Biomaterials that can undergo resorption by osteoclasts, the first step in bone remodeling, are gradually replaced by new bone tissue, as osteoclast resorption is followed by deposition of new matrix by osteoblasts.[5,6] Although bone graft substitutes based on biomineralized collagen closely resemble the physicochemical properties of bone tissue, currently, limited evidence exists that these materials can undergo osteoclastdriven degradation.

In a previous study, we found that osteoclasts were able to form from human monocyte-macrophage precursors on biomineralized collagen membranes; however, they were not capable of efficiently forming sealing zones and resorbing the biomineralized membrane (submitted manuscript). We hypothesized that a combination of material properties was responsible for this lack of active resorption. First, the porous, mesh-like structure of the membranes possibly inhibited the sealing of a compartment for resorption. Second, the membranes exhibited a 
reduced stiffness when compared to other, frequently used substrates for in vitro study of osteoclast resorption, such as dentin or cortical bone slices, possibly affecting the resorptive activity of osteoclasts. Finally, the absence of native RGD ligands in the membrane (thus not originating from cell culture medium components) to the vitronectin receptor may have been a reason that no osteoclastic resorption of biomineralized collagen membranes occurred. ${ }^{[7,8]}$

With the goal of triggering osteoclastic resorption, in this study, we propose a modification of a biomineralized collagen membrane by depositing an additional, cobalt-containing $\mathrm{CaP}$ coating on its surface. The rationale behind this modification was two-fold. First, by increasing the amount of mineral surrounding the collagen fibers (extrafibrillar mineral), the porosity of the membrane is decreased, possibly enabling osteoclasts to seal a compartment for resorption. Second, the addition of bioactive cobalt ions $\left(\mathrm{Co}^{2+}\right)$ may increase osteoclast activity. Presence of cobalt ions has previously been shown to affect the osteoclast behavior, inducing higher resorptive activity at concentrations around the micromolar range. ${ }^{[9]}$

The addition of trace amounts of $\mathrm{Co}^{2+}$ via an extrafibrillar $\mathrm{CaP}$ coating is an alternative way of introducing guest inorganic ions in biomaterials based on biomineralized collagen, as cations are usually difficult to incorporate in the intrafibrillar mineral fraction. Indeed, different cations, such as $\mathrm{Cu}^{2+}$ and $\mathrm{Sr}^{2+}$ have been shown to inhibit the formation of intrafibrillar crystals due to disruption of the polymer-induced liquid precursor (PILP) method, which is commonly used for intrafibrillar mineralization of collagen. ${ }^{[10,11]}$ More generally, incorporation of small amounts of bioactive inorganic ions is an attractive strategy to affect processes related to bone formation and remodeling, and is interesting also from the perspective of producing closer-to-native materials, as trace amounts of various metallic ions are present in bone apatite. ${ }^{[12]}$

Here we produced and characterized biomineralized collagen membranes modified by deposition of a Co-containing CaP coating, and explored the effect of this modification on the formation and function of osteoclasts derived from human peripheral blood. 


\section{Materials and Methods}

\subsection{Materials}

PureCol ${ }^{\circledR}$ collagen type-I solution was purchased from Advanced BioMatrix (San Diego, California, USA, cat\#5005). Poly-L-aspartic acid sodium salt ( $\mathrm{pAsp}, \mathrm{Mw}=27$ $\mathrm{kDa}$ ) was purchased from Alamanda Polymers (Huntsville, Alabama, USA, cat\#000D200). 1-Ethyl-3-[3-dimethylaminopropyl] carbodiimide hydrochloride (EDC), Nhydroxysulfosuccinimide (sulfo-NHS), calcium chloride dihydrate, potassium phosphate dibasic, cobalt chloride hexahydrate and all other chemicals were obtained from Sigma-Aldrich (St. Louis, Missouri, USA). Recombinant human MCSF and soluble RANK-L cytokines were from Peprotech (Connecticut, USA, cat\#3002510 and cat\#3100110). The $\beta$-TCP ceramic discs were kindly supplied by Kuros Biosciences. The $\beta$-TCP was prepared as described previously, ${ }^{[13]}$ and machined in the shape of discs with a diameter of $9 \mathrm{~mm}$ and a thickness of $1 \mathrm{~mm}$.

\subsection{Preparation of biomineralized collagen membranes}

Biomineralized collagen membranes were prepared as previously described.[14,15] Briefly, collagen was mixed with 10x concentrated phosphate buffered saline (PBS) and $0.1 \mathrm{M} \mathrm{NaOH}$, in a volume fraction of, respectively: 0,$726 ; 0,246 ; 0,118$. Volumes of $2 \mathrm{~mL}$ were gelled in a humidified incubator at $37^{\circ} \mathrm{C}$ for $24 \mathrm{~h}$. These gels were then placed on top of a nylon mesh and incubated for $48 \mathrm{~h}$ to reduce water content, which resulted in the formation of flat membranes. Next, the membranes were cross-linked overnight at room temperature in $50 \mathrm{mM}$ MES buffer $(\mathrm{pH}=7.0)$, containing $50 \mathrm{mM}$ EDC and $25 \mathrm{mM}$ sulfo-NHS, before proceeding with the intrafibrillar mineralization of the collagen. This was achieved with the PILP method, by incubating the membranes for 7 days at $37^{\circ} \mathrm{C}$ in a $50 \mathrm{mM}$ Tris buffer $(\mathrm{pH}=7.4)$ containing $150 \mathrm{mM}$ $\mathrm{NaCl}, 4.5 \mathrm{mM} \mathrm{CaCl}, 2.1 \mathrm{mM} \mathrm{K} \mathrm{HPO}_{4}$ and $100 \mu \mathrm{g} / \mathrm{mL}$ pAsp. All steps were performed in a sterile environment.

\subsection{Cobalt-containing calcium phosphate coating (CoCaP coating)}

The already (intrafibrillary) mineralized collagen membranes underwent a two-step coating procedure. In a first step, membranes were incubated in a $2.5 x$ concentrated simulated body fluid (SBF) solution, which was prepared by mixing 3 precursor solutions: buffer, calcium stock and phosphate stock solution, in a ratio of 2:1:1. 
Buffer solution consisted of $50 \mathrm{mM}$ Tris ( $\mathrm{pH}=7.4)$; calcium stock solution consisted of $25 \mathrm{mM} \mathrm{CaCl}_{2}, 1.37 \mathrm{M} \mathrm{NaCl}$ and $15 \mathrm{mM} \mathrm{MgCl}_{2}$ (in buffer solution); and phosphate stock solution consisted of $11.1 \mathrm{mM} \mathrm{Na}_{2} \mathrm{HPO}_{4}, 42 \mathrm{mM} \mathrm{NaHCO}_{3}$ (in buffer solution). The membranes were placed into 24-well plates, incubated in $2 \mathrm{~mL}$ of $2.5 \times \mathrm{SBF}$ for 3 days at room temperature and finally washed with distilled water. In the second step, the membranes were again placed into 24-well plated and incubated for 3 days at room temperature in $2 \mathrm{~mL}$ of a calcium phosphate solution consisting of $50 \mathrm{mM}$ Tris ( $\mathrm{pH}=7.4), 4 \mathrm{mM} \mathrm{CaCl}_{2}$ and $2.25 \mathrm{mM} \mathrm{Na}_{2} \mathrm{HPO}_{4}$, to which a cobalt stock solution of $1 \mathrm{mM} \mathrm{CoCl}_{2}$ (in distilled water) was added to achieve final concentrations of 0.1 , 1,5 , and $10 \mu \mathrm{M}$, respectively. The membranes were then washed with distilled water and prepared for characterization or cell culture. All coating steps were performed in a sterile environment.

\subsection{Thermogravimetric analysis}

Thermogravimetric analysis was performed on the uncoated and CoCaP-coated biomineralized collagen membranes $(n=3)$. Membranes were washed with MilliQ water and blotted with filter paper before being heated up to $800{ }^{\circ} \mathrm{C}$, at a rate of 5 ${ }^{\circ} \mathrm{C} / \mathrm{min}$, using a Q500 TGA (TA Instruments, Belgium). The leftover weight at $800{ }^{\circ} \mathrm{C}$ was considered the mineral content of the membrane.

\subsection{Nanoindendation measurements}

Nanoindentation measurements were performed in wet state on the uncoated and CoCaP-coated biomineralized collagen membranes, using a Piuma Nanoindenter (Optics11 Life, the Netherlands), which was equipped with a $51.11 \mathrm{k}(\mathrm{N} / \mathrm{m})$ probe with a tip radius of $24.5 \mu \mathrm{m}$ (ref\# P190504). Probe calibration was performed using a glass slide submerged in distilled water. Measurements were made in "displacement control" mode, where the tip presses into the substrate until a certain probe displacement is reached (set at $10 \mu \mathrm{m}$ ). An area composed of $4 \times 4$ points was defined on each membrane, with measurement points ( $\mathrm{n}=16$ from one sample) every $200 \mu \mathrm{m}$. The Oliver and Pharr method was used to fit the raw data and estimate the elastic modulus. To ensure a flat and stable surface, membranes were bonded to the bottom of a plastic petri dish using double-sided tape, and covered with distilled water. 
2.6. Monocyte/macrophage culture and osteoclast differentiation Poietics $^{\mathrm{tm}}$ human peripheral blood CD14+ monocytes were obtained from Lonza (Basel, Switzerland, cat\#2W-400B, lot\#647890). Upon thawing the cryovial, cells were transferred to a $15 \mathrm{~mL}$ tube containing $9 \mathrm{~mL}$ warm basic cell culture medium $(\alpha-$ MEM (Lonza, cat\#BE02-002F) supplemented with $10 \%(\mathrm{v} / \mathrm{v})$ HyClone $^{\text {tm }}$ FetalClone $^{\text {tm }}$ serum (Thermo Fisher Scientific, USA, cat\#10780245, lot\#HXSH3008003), 100 U/ml penicillin, $100 \mu \mathrm{g} / \mathrm{ml}$ streptomycin and $20 \mathrm{ng} / \mathrm{mL} \mathrm{M-CSF}$ ), followed by centrifugation at $300 \mathrm{rcf}$ for $5 \mathrm{~min}$. Cells were re-suspended in basic cell culture medium, counted, and seeded at a density of 500.000 cells $/ \mathrm{cm}^{2}$ on cortical bone slices inside 96-well plates or on the uncoated and CoCaP-coated biomineralized collagen membranes inside 48-well plates. For the first 3 days of culture, the basic medium was modified to contain $35 \mathrm{ng} / \mathrm{mL}$ M-CSF. Then, the culture continued in either basic (20 ng/mL M-CSF) or in differentiation medium (basic medium supplemented with $40 \mathrm{ng} / \mathrm{mL}$ RANK-L) for up to 21 days, counting from the addition of RANK-L, with medium refreshment every 2-3 days. Before cell seeding, all substrates were incubated for $4 \mathrm{~h}$ with basic medium (without M-CSF). All steps were performed in a sterile environment.

\subsection{Confocal laser microscopy}

Uncoated and coated membranes and cortical bone slices with cells $(n=2)$ were fixed with warm 10\% formaldehyde in PBS (15 min at room temperature) for fluorescence staining after 14, or 21 days of culture. The samples were washed three times with PBS, and permeabilized with $0.2 \%(\mathrm{v} / \mathrm{v})$ Tween-20 in PBS for 10 minutes at room temperature. For visualization of tartrate-resistant alkaline phosphatase (TRAP) activity, the samples were incubated with ELF-97 phosphate substrate (Thermo Fisher Scientific, USA, cat\#E6601) according to the staining method by Filgueira. ${ }^{[16]}$ Briefly, 20x concentrated ELF-97 substrate was diluted in a buffer composed of 110 $\mathrm{mM}$ acetate ( $\mathrm{pH}=5.2), 1.1 \mathrm{mM}$ sodium nitrite and $7.4 \mathrm{mM}$ tartrate, and samples were incubated for $15 \mathrm{~min}$. For staining of F-actin, samples were incubated with 1:200 PBSdiluted AlexaFluor ${ }^{\circledR} 488$ phalloidin (Thermo Fisher Scientific) for $20 \mathrm{~min}$, and finally for staining nuclei, incubated with $1 \mu \mathrm{g} / \mathrm{mL}$ DAPI (Thermo Fisher Scientific, USA, cat\#1306) in PBS for $10 \mathrm{~min}$. All incubation steps were at room temperature, in the dark. Imaging was done with a SP8 STED confocal laser microscope (Leica Microsystems, Germany) using 25x and 86x water-immersion objectives. Large, 
multinucleated ( $\mathrm{n}>2$ ) and TRAP-positive cells were considered to be osteoclasts. Quantification of the TRAP activity ${ }^{1}$ was performed by image analysis using Image $\mathrm{J}$ (version 1.52r). Briefly, the number of TRAP-positive pixels was counted for each osteoclast, and expressed as fraction of total cell area. Details of this analysis are provided in a short video as a part of the available dataset published with this study.

\subsection{Scanning electron microscopy (SEM)}

For SEM morphological characterization, both the uncoated and CoCaP-coated biomineralized collagen membrane were dehydrated in a series of ethanol in water $(30 \%, 40 \%, 50 \%, 60 \%, 70 \%, 80 \%, 90 \%, 100 \%), 15$ min per step, followed by $30 \mathrm{~min}$ in hexadimethylsiloxane before drying overnight. The membranes with cells underwent the same treatment, but preceded by a cell-lysis step with $1 \%$ Triton X100 in PBS for $30 \mathrm{~min}$ in an ultrasonic waterbath, with ice. After dehydration, samples were glued onto aluminum stubs with carbon tape and silver paint, and sputter-coated with a $2 \mathrm{~nm}$ layer of iridium using a Q150TES sputter coater (Quorum, United Kingdom). Imaging was performed on a TENEO SEM (FEI, OR, United States) using the T1 in-column or back-scatter detectors in Optiplan mode, with the beam at $2-10 \mathrm{kV}$ and working distance of $2-10 \mathrm{~mm}$.

\subsection{Ion-coupled plasma mass spectrometry (ICP-MS)}

To determine the total amount of $\mathrm{Co}^{2+}$ incorporated in the membranes for different starting concentrations of $\mathrm{CoCl}_{2}$ used in the coating solution, membrane samples with a diameter of $6 \mathrm{~mm}(\mathrm{n}=3)$ were dissolved overnight in $2 \mathrm{~mL}$ ultra-pure $60 \%$ $\mathrm{HNO}_{3}$. Samples were then diluted with MilliQ water to a final concentration of $1 \%$ $\mathrm{HNO}_{3}$, and $100 \mu \mathrm{L}$ of diluted sample were mixed with $900 \mu \mathrm{L}$ of measuring matrix ( $1 \% \mathrm{HNO}_{3}$ with $20 \mathrm{ppb}$ scandium). A standard curve of calcium (ranging from 100 to $4000 \mathrm{ppb}$ ) and cobalt (ranging from 6 to $200 \mathrm{ppb}$ ) was used to quantify the concentrations. Data is presented as molar ratio $\mathrm{Co} / 10^{6} \mathrm{Ca}$ to normalize for the calcium content of each replicate.

\footnotetext{
${ }^{1}$ Here it should read semi-quantification, i.e. the comparison of amounts of fluorescent substrate (ELF-97) converted by TRAP between different conditions. The type of image analysis presented in this chapter is not sufficient to determine the activity of the TRAP enzyme. We ask the reader to keep this in mind while analyzing Figures 2 and 3 as well as their corresponding discussion. The original text was kept as published in Biomaterials Research and this note was added for clarification.
} 
For quantification of $\mathrm{Co}^{2+}$ release from $\mathrm{CoCaP}$-coated membranes, membrane samples with a diameter of $6 \mathrm{~mm}(\mathrm{n}=3)$ were incubated with MilliQ water $(150 \mathrm{uL}$ in a 96-well plate). Supernatant was collected at designated time-points, stored in micro centrifuge tubes at $-30^{\circ} \mathrm{C}$, and fresh MilliQ was added to the samples. At the end of the experiment, collected supernatants were thawed, and $100 \mu \mathrm{L}$ sample was added to $900 \mu \mathrm{L}$ of measuring matrix ( $1 \% \mathrm{HNO}_{3}$ with $20 \mathrm{ppb}$ scandium). A standard curve of cobalt (ranging from 6 to $200 \mathrm{ppb}$ ) was used to quantify the amount of released ions. Data is presented as the cumulative $\mathrm{Co}^{2+}$ concentration over the entire duration of the experiment.

Quantification of $\mathrm{Ca}^{2+}$ released during cell culture was performed for cells cultured on either uncoated or CoCaP-coated collagen membranes, in either basic or differentiation medium ( $n=3$ per condition). At every medium refreshment time point, cell culture supernatant was collected in micro centrifuge tubes and the tubes were kept at $-30^{\circ} \mathrm{C}$ until collection of all samples was completed. Then, the collected medium was thawed, and $50 \mu \mathrm{L}$ sample was added to $950 \mu \mathrm{L}$ of measuring matrix ( $1 \% \mathrm{HNO}_{3}$ with $20 \mathrm{ppb}$ scandium). A standard curve of calcium ranging from 100 to $4000 \mathrm{ppb}$ was prepared, and $20 \mathrm{ppb}$ scandium was used as internal standard in all samples measured, to compensate signal drift from the instrument, as well as other factors.

\subsection{Statistical analysis}

Statistical analysis was performed in GraphPad Prism (version 8.3). The Student's ttest was used to compare stiffness data from nanoindentation measurements, as well as TGA data. Two-way analysis of variance was used to compare $\mathrm{Ca}^{2+}$ concentration data from ICP-MS and TRAP activity in which differences in means between conditions were tested within each timepoint. A Bonferroni post-hoc test was used to correct for multiple comparisons (one family for all comparisons). Mean differences were considered statistically significant for $p$-value $<0.05$. In all figures, the following notation applies: ${ }^{*} \mathrm{p}<0.05 ;{ }^{* *} \mathrm{p}<0.001$; ${ }^{* *} \mathrm{p}<0.0001$. 


\section{Results}

In this study, we aimed at developing a biomineralized collagen membrane that allows the differentiation of osteoclasts from human peripheral blood monocytes and osteoclastic resorption, by depositing a $\mathrm{CoCaP}$ coating on the mineralized collagen fibrils of the membrane.

\subsection{CoCaP coating increases stiffness of biomineralized collagen membrane}

Biomineralized collagen membranes (BiominCol in figures) were prepared using a modified PILP method as described previously.[14,15] The membranes had a fibrillar surface structure, as observed by SEM (Figure 1A). Incubation of the membranes in a $\mathrm{CaP}$ solution containing $\mathrm{Co}^{2+}$ did not lead to apparent changes of the surface structure, and no mineral deposits were observed (Figure 1B). TGA results showed that the mass remaining after combustion of the organic phase of the membranes increased from $67 \%(\mathrm{w} / \mathrm{w})$ for the uncoated collagen membrane to $71 \%(\mathrm{w} / \mathrm{w})$ for the CoCaP-coated one (Figure 1C), indicating that the CoCaP coating slightly increased the mineral content of the membrane. The ICP-MS results showed that increasing concentrations of $\mathrm{Co}^{2+}$ in the $\mathrm{CaP}$ coating solution, caused corresponding increase in the amount of $\mathrm{Co}^{2+}$ detected after dissolution of the coated membranes in acid, which confirmed that Co-containing mineral was deposited in the membrane (Figure 1D). For a starting concentration of $1 \mu \mathrm{M} \mathrm{Co}^{2+}$ there were $20 \mathrm{Co}^{2+}$ per million $\mathrm{Ca}^{2+}$. This formulation was chosen for all other experiments in this study. CoCaP-coated collagen membranes showed a continuous release of $\mathrm{Co}^{2+}$ in water over a period of 16 days (Figure 1E), with an average release of approximately 400 ppt per refreshment period (2-3 days), which is equivalent to a molarity of around $7 \mathrm{nM}$. Nanoindentation measurements further showed that the stiffness of the CoCaPcoated collagen membrane (about $10 \mathrm{MPa}$ ) was about one order of magnitude higher relative to the uncoated membrane (Figure $1 \mathrm{~F}$ ), which is likely a result of the presence of additional mineral. 
A

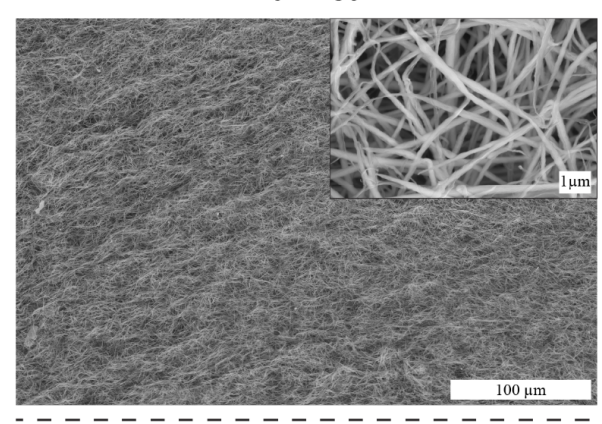

B

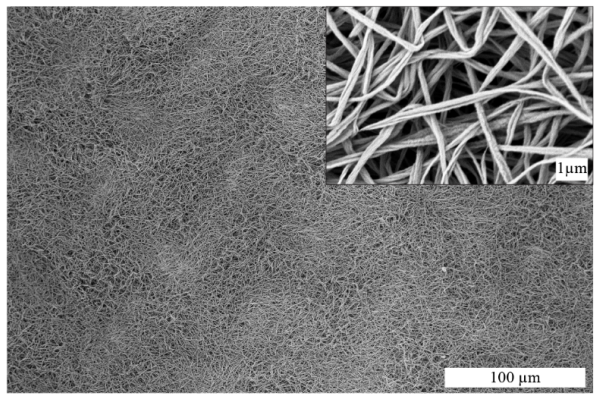

C) TGA

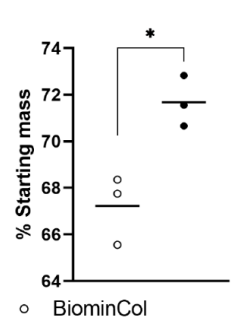

- CoCaP-coated BiominCol
D) Incorporation of $\mathrm{Co}^{2+}$

E) Release of $\mathrm{Co}^{2+}$

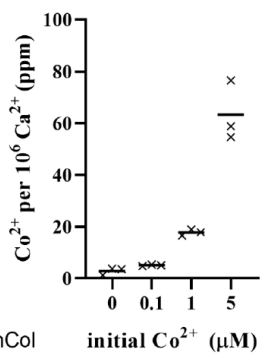

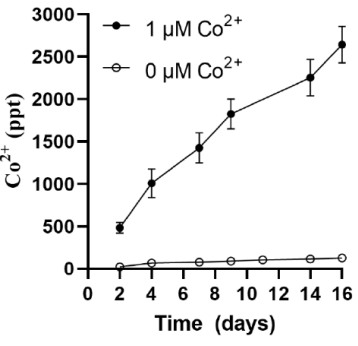

F) Stiffness

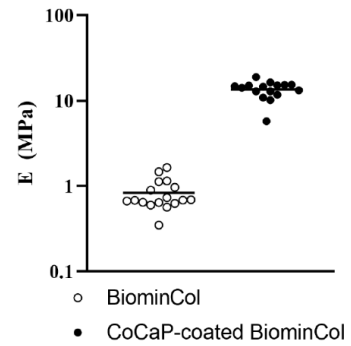

Figure 1. Characterization of uncoated and CoCaP-coated biomineralized collagen (BiominCol) membranes. SEM images of the surface topography of uncoated and CoCaPcoated BiominCol show that both materials have a fibrillar structure. No mineral deposits are seen on the CoCaP-coated BiominCol membrane and the mesh-like structure seems to be preserved after the coating procedure, without obvious differences in fibril diameter. Quantification of $\mathrm{Co}^{2+}$ by ICP-MS in CoCaP-coated BiominCol membranes, expressed as the ratio of $\mathrm{Co}^{2+}$ to $\mathrm{Ca}^{2+}$ for $\mathrm{CoCaP}$ coating solutions with different initial $\mathrm{Co}^{2+}$ concentrations. The initial concentration of $1 \mu \mathrm{M}$ was chosen for the rest of the study. Quantification of $\mathrm{Co}^{2+}$ released by $\mathrm{CoCaP}$-coated BiominCol membranes in water, expressed as cumulative release over a period of 16 days $(n=3)$. Quantification of mineral content of uncoated and CoCaPcoated BiominCol membranes by TGA, expressed as the remaining mass after burning at 800 ${ }^{0} \mathrm{C}$, showing an about $4 \%(\mathrm{w} / \mathrm{w})$ higher mineral content in CoCaP-coated membranes. Elasticity modulus determined by nanoindentation measurements of uncoated and CoCaPcoated BiominCol. The CoCaP coating increased the membrane modulus of elasticity by about one order of magnitude $(\mathrm{p}<0.0001)$. 
3.2. Osteoclasts differentiate from monocytes and show actin rings on CoCaPcoated mineralized collagen membranes, but not on uncoated membranes

Monocytes derived from human peripheral blood were cultured on uncoated and CoCaP-coated biomineralized collagen membranes, and stimulated to differentiate into osteoclasts by the addition of $40 \mathrm{ng} / \mathrm{mL}$ RANK-L to basic cell culture medium. After 7 days in differentiation medium, a few large and multinucleated cells were observed on the uncoated membranes (Figure 2A), but not on CoCaP-coated membranes (Figure 2B). TRAP-positive cells were not observed on either of the membranes after 7 days, but were present on both membranes after 14 and 21 days. Quantification of TRAP activity by image analysis showed that on average, TRAP activity of osteoclasts on CoCaP-coated membranes was twice as high as that on uncoated membranes at 14 and 21 days $(p=0.01)$ (Figure 3 ). After 14 days of culture, osteoclasts on both uncoated and coated membrane showed concentrated actin structures (Figure 2C-D). These actin structures were dispersed through the cell and did not form clear actin rings. After 21 days of culture in differentiation medium, a few osteoclasts on the CoCaP-coated membranes exhibited circular, high-intensity actin structures that resemble actin rings (Figure 2F). It should however be noted that the number of osteoclast with clearly defined actin rings was limited. These structures were not detected on the uncoated biomineralized collagen membrane (Figure 2E).

Figure 2. (next page) Confocal laser microscopy images (top and side view) of osteoclasts cultured on uncoated (A, C, E) and CoCaP-coated BiominCol membranes (B, D, F), at 7, 14, and 21 days. At 7 days, multi-nucleated cells were visible on uncoated BiominCol, with the central cell showing a thick actin structure (podosome belt - dashed circle). CoCaP-coated BiominCol membrane also exhibited multi-nucleated cells at this point. No TRAP+ cells were observed on either substrate. At 14 days, several TRAP+ multi-nucleated large cells were observed on both surfaces. High intensity actin structures were observed close to the surface of the material, but they do not appear to be actin rings. At 21 days, actin structures resembling actin rings were observed on some osteoclasts on CoCaP-coated BiominCol membranes (dashed circle and arrowheads), but not on the uncoated ones. Dashed lines in the side views represent the surface of the membrane. For all images: scale bar is $50 \mu \mathrm{m}$, actin in red, nuclei in blue, and TRAP in green. 
BiominCol
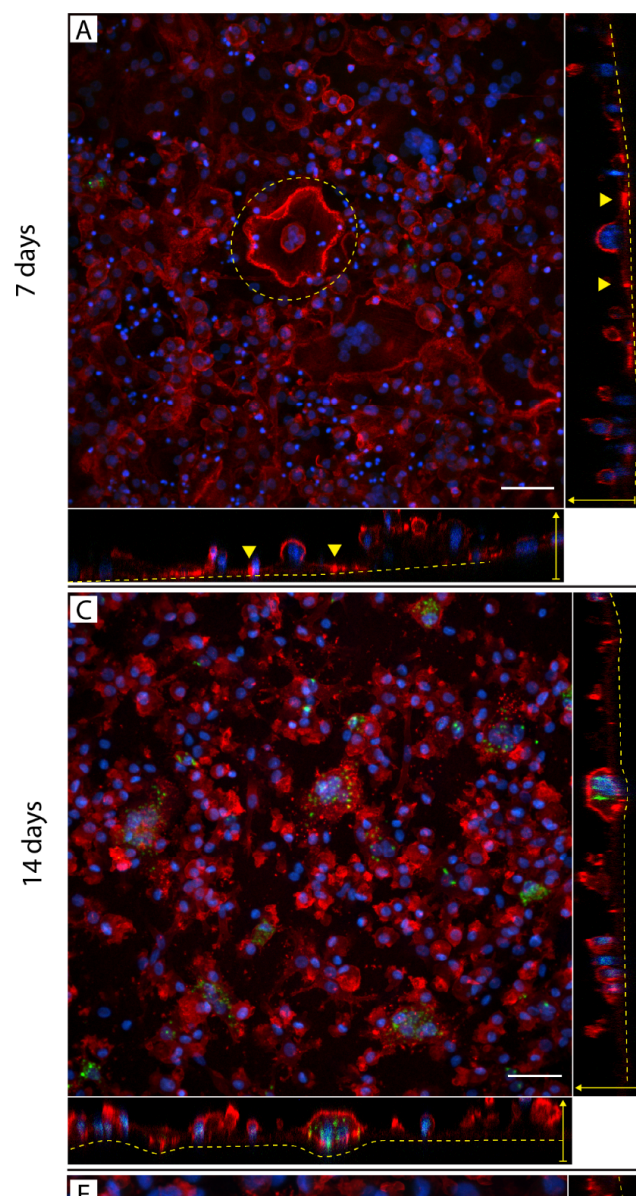

CoCaP-coated BiominCol

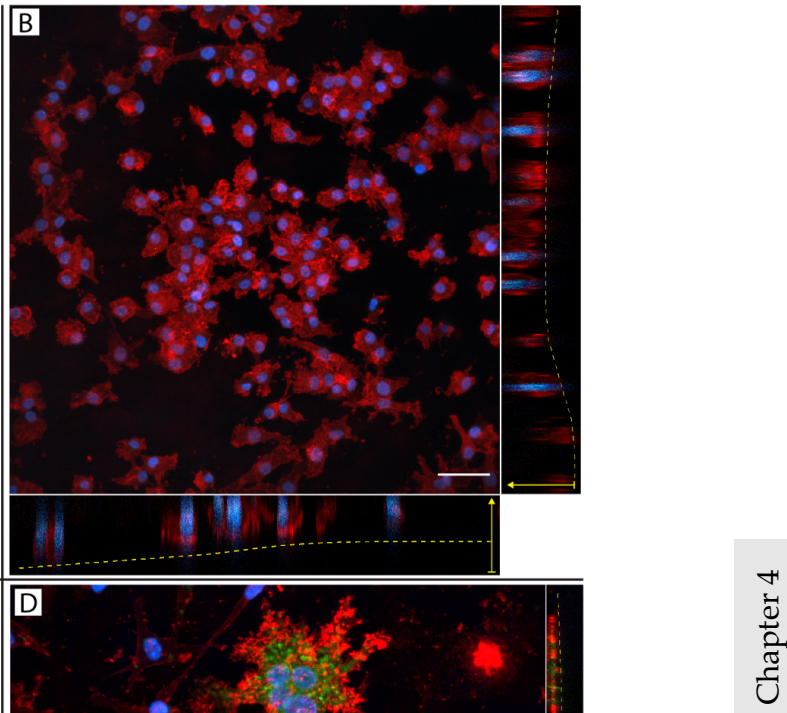




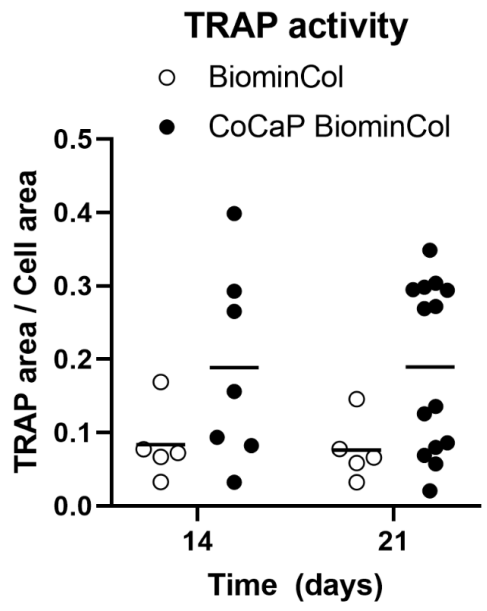

Figure 3. Semi-quantitative comparison of TRAP activity between osteoclasts cultured on BiominCol and CoCaP-coated BiominCol. TRAP activity is shown as the area fraction of TRAP+ pixels relative to the whole cell area, for each osteoclast (each point represents one osteoclast).

3.3. Presence of Co and increased stiffness stimulate limited resorption of the coated biomineralized collagen membrane

Resorption of uncoated and $\mathrm{CoCaP}$-coated biomineralized collagen membranes was evaluated by measuring the $\mathrm{Ca}^{2+}$ concentration in the cell culture medium, up to 21 days (Figure 4). This method allows for indirect quantification of resorptive activity, and is suitable for materials for which quantification of resorbed area or volume by conventional imaging methods is difficult, for example because of the high roughness or porosity of the substrate as is the case for collagen membranes. Nevertheless, a limitation of this method is a relatively low sensitivity; as the $\mathrm{Ca}^{2+}$ concentration in medium varies with many processes associated with cell culture, only major differences in resorptive behavior can be quantified. For example, when biomineralized collagen is immersed in cell culture medium in the absence of cells, there is a depletion of $\mathrm{Ca}^{2+}$ from the medium (data not shown). Nevertheless, the substrate-based effects are accounted for in the results as a comparison is only made between cells cultured in basic versus differentiation medium, on the same substrate. Therefore, any difference in $\mathrm{Ca}^{2+}$ concentration should be a result of cell differentiation and resorptive activity. 
On the uncoated membranes, no significant differences in $\mathrm{Ca}^{2+}$ concentration were observed between cultures in basic and differentiation medium, except at the last time point of 21 days, when there was a significantly higher $\mathrm{Ca}^{2+}$ content in the basic medium condition (Figure 4A). This is an unexpected finding, as no $\mathrm{Ca}^{2+}$ release should occur from the substrate in the absence of cells, or from macrophage activity. On the CoCaP-coated membrane, there were significant differences in $\mathrm{Ca}^{2+}$ concentration between basic and differentiation medium at 2, 9 and 16 days, with differentiation medium having a higher $\mathrm{Ca}^{2+}$ content (Figure 4B).

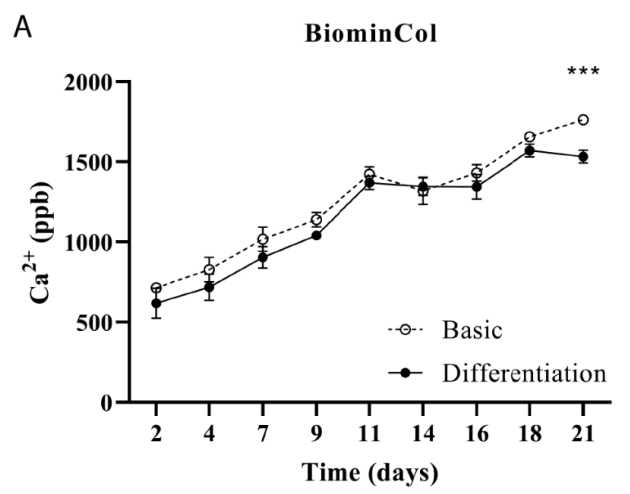

B

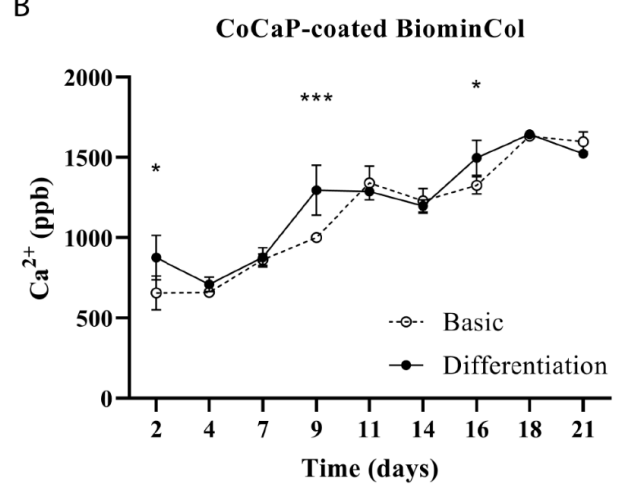

Figure 4. Quantification of $\mathrm{Ca}^{2+}$ in cell culture medium by ICP-MS over a period of 21 days of cell culture in basic or differentiation medium. (A) For uncoated BiominCol membranes, no major differences between the basic and differentiation medium were observed, indicating that resorption by osteoclasts was limited or non-existent. (B) For CoCaP-coated BiominCol membranes, a significantly higher $\mathrm{Ca}^{2+}$ concentration was seen for the differentiation condition on day $2(p=0.37)$, day $9(p<0.001)$ and day $16(\mathrm{p}=0.016)$.

To further investigate whether the differences in $\mathrm{Ca}^{2+}$ concentration were due to osteoclast resorption, the same uncoated and CoCaPcoated membranes for which the

ICP-MS analysis of the medium was performed, were observed by SEM, after removing the cells. On uncoated biomineralized collagen membranes, no obvious signs of resorption were observed (Figure 5A). There were areas on the membrane where the fibrillar structure appeared to be disturbed, which probably correspond to places of cell attachment (Figure 5C). On the CoCaP-coated membranes, the same areas with disrupted fibrillar structure were also present, but interestingly, some of 
these areas also exhibited resorption lacunae-like features, i.e., pockets indented in the surface of the biomaterial (Figure 5B, 5D).

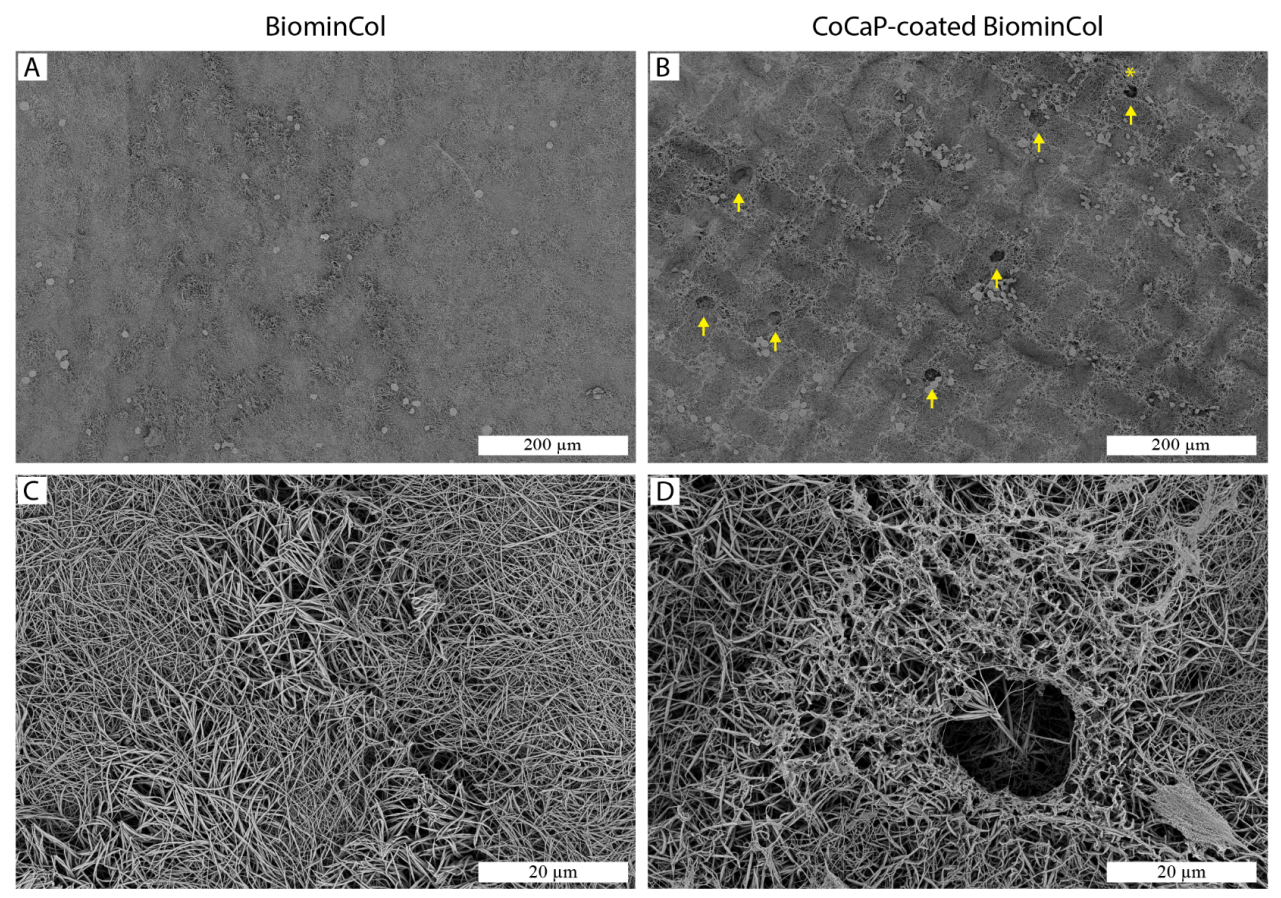

Figure 5. SEM images of uncoated (A, C) and CoCaP-coated BiominCol membranes (B, D) after 21 days of cell culture. Cells were removed prior to imaging. Both substrates showed signs of disruption of intrafibrillar membrane structure by cells. On the CoCaP-coated BiominCol membrane, rounded depressions with a diameter of about $20 \mu \mathrm{m}$ were observed that resemble resorption lacunae (yellow arrows).

\section{Discussion}

\subsection{CoCaP coating increases stiffness of biomineralized collagen membrane}

The modified PILP method, followed by a coating method was used to develop $\mathrm{CoCaP}$-coated biomineralized collagen membrane. The absence of apparent mineral deposits on the surface (Figure 1B) after incubation of the biomineralized collagen membrane in the CoCaP solution was unexpected, as the TGA data showed a $4 \%$ $(\mathrm{w} / \mathrm{w})$ increase in mineral content after the coating process (Figure 1C). Moreover, previous studies have shown that $\mathrm{CaP}$ nodules (spherulitic mineral aggregates) 
were deposited on tissue culture plastic upon immersion in $\mathrm{CaP}$ solutions similar to the one used here, with or without $\mathrm{CO}^{2+.}{ }^{217,18]}$ It has also been shown that $\mathrm{CaP}$ mineral deposits formed on collagen scaffolds upon incubation into concentrated SBF solutions. ${ }^{[19-21]}$

A few aspects related to the properties of the biomineralized collagen membrane and the CaP coating method used here may explain why this extra mineral deposition, originating from the coating, was not observed on SEM images. First, the surface area of the mineralized collagen membranes was much higher than that of cell culture plates used in the earlier studies, ${ }^{[17,18]}$ which means that the same amount of mineral that would normally deposit on a flat surface could now form on many more available nucleation/growth sites, resulting in the formation of smaller mineral particles. Second, the CoCaP coating step provided less total mineral than, for example, the PILP method used for intrafibrillar collagen mineralization; the CoCaP coating solution had about ten times lower ion content than the PILP solution, due to the lower reaction volume used, as described in the methods section. This may explain why there was no noticeable fiber diameter increase after the coating step (uncoated BiominCol at $188 \pm 45 \mathrm{~nm}$ and CoCaP-coated BiominCol at $156 \pm 30, \mathrm{n}=$ 20), in contrast to the observed fiber diameter increase following the intrafibrillar mineralization. ${ }^{[14,15]}$ Finally, and perhaps most importantly, the biomineralized collagen membranes used in our study already contained intrafibrillar $\mathrm{CaP}$ mineral before immersion into the $\mathrm{CoCaP}$ coating solution, which may have interfered with nucleation and growth of $\mathrm{CaP}$ deposits on the fibrous collagen surface. It is suggested that $\mathrm{CoCaP}$ coating preferentially crystallized on the pre-existing intrafibrillar CaP. This seems plausible based on earlier studies that compared the rate of hydroxyapatite (HA) crystal growth on collagen, elastin and HA surfaces. These studies showed that the growth rate of HA on the HA surface was several orders of magnitude higher than on the polymeric substrates. ${ }^{[22-24]}$ It should be noted that we tried to incorporate $\mathrm{Co}^{2+}$ intrafibrillarly, by adding the ion to the PILP solution. This was, however, unsuccessful as no intrafibrillar mineral was formed. This result is in accordance with previous reports in which inhibitory effects of cations such as $\mathrm{Cu}^{2+}{ }^{[10]}$ and $\mathrm{Sr}^{2+}{ }^{[1]}$ on the PILP-induced biomineralization were observed.

Taken together, based on the SEM characterization of the structural properties of mineralized collagen membranes before and after immersion in the CoCaP coating 
solution, there was no evidence for the deposition of the mineral on the surface; however, it is suggested that the new mineral deposited on the pre-existing intrafibrillar mineral, which could not be observed using SEM imaging. This hypothesis was supported by the TGA data, presented above, as well by the results of the ICP-MS analysis of the $\mathrm{Co}^{2+}$ content of coated membranes, measured for different initial concentrations of $\mathrm{Co}^{2+}$ in the solution and presented as the number of $\mathrm{Co}^{2+}$ per $10^{6} \mathrm{Ca}^{2+}$ (Figure 1D).

The additional $4 \%(\mathrm{w} / \mathrm{w})$ mineral content, formed during the coating process, is likely responsible for the increased stiffness of the CoCaP-coated collagen membrane (Figure 1F). It should be noted that both the uncoated and CoCaP-coated collagen membranes prepared here were significantly less stiff than the biomineralized collagen membrane reported in an earlier study (177 MPa).[15] Although a similar biomineralization method was used in both studies, differences in fiber density and density of crosslinks between the materials obtained in the two studies may explain different mechanical properties.

The TGA, ICP-MS, and nanoindentation data showed that the CoCaP coating process changed the physicochemical properties of the substrate, by incorporation of Co in its composition and by increasing its stiffness, without significantly altering the surface morphology of the biomineralized collagen membrane.

\subsection{Osteoclasts differentiate from monocytes and show actin rings on CoCaP- coated mineralized collagen membranes, but not on uncoated membranes}

No multinucleated TRAP+ cells were observed on either type of membrane at day 7 , but at day 14 and 21, osteoclasts were observed on both uncoated and CoCaP-coated biomineralized collagen membranes (Figure 2C-F), and TRAP activity was on average twice as high on the CoCaP-coated membranes (Figure 3). Moreover, osteoclasts showing actin rings were only observed on the CoCaP-coated membranes (Figure 2F). Intracellular TRAP activity was previously shown to correlate with resorption of CaP cements, while correlation between extracellular TRAP activity and resorption was reported for dentin and osteoblast-produced matrix. ${ }^{[25]}$ Further research is needed to investigate whether the more pronounced TRAP activity on CoCaP-coated mineralized collagen membranes observed here is indicative of the coated membranes being resorbed by osteoclasts. 
In an earlier study, we have shown that biomineralized collagen membranes supported osteoclast formation. Nevertheless, the formed osteoclasts were unable to form stable actin rings or sealing zones, and were therefore incapable of resorption. Here, we observed for the first time osteoclasts with actin rings on the CoCaP-coated biomineralized collagen membranes. As was described in the previous section, the coated membranes contained additional $\mathrm{CaP}$ with $\mathrm{Co}^{2+}$ and had increased stiffness relative to the uncoated ones. Both modifications may have contributed to the difference in osteoclast phenotype, i.e., the capacity to form actin rings, but it is likely that the increased stiffness is the main factor. Few studies investigated the effects of $\mathrm{Co}^{2+}$ on formation and resorptive activity of osteoclasts in the similar concentration range as used here. In a study by Patntirapong et al., surface of tissue culture well plates was coated with thin $\mathrm{CaP}$ layers containing $\mathrm{Co}^{2+}$ using solutions with a $\mathrm{Co}^{2+}$ concentration of $0.1,1$, and $5 \mu \mathrm{M}$, respectively. These coatings, which were shown to release about 1,10 , and $50 \mathrm{ng}$ of $\mathrm{Co}^{2+}$ in $500 \mu \mathrm{L}$ cell culture medium, respectively over a period of 3 days, supported the formation of a higher number of murine osteoclasts $(1 \mu \mathrm{M}$ condition in particular), and larger resorbed areas (all three conditions). ${ }^{[9]}$ For comparison, after 2-day incubation in water of the CoCaP-coated collagen membrane used in our study, the $\mathrm{Co}^{2+}$ concentration was around $0.4 \mu \mathrm{g} / \mathrm{L}$ (equivalent to around $7 \mathrm{nM}$ ), which is lower than the $\mathrm{Co}^{2+}$ released in the $0.1 \mu \mathrm{M}$ condition of the above discussed study ( $2 \mathrm{ug} / \mathrm{L}$ ). Another study showed that $10 \mathrm{nM}$ of dissolved $\mathrm{Co}^{2+}$ had either no effect on the resorptive activity of forming human osteoclasts, or caused a slight drop in the resorptive activity of mature osteoclasts. ${ }^{[26]}$ Taken together, the CoCaP-coated collagen membranes used in our study contained a lower amount of $\mathrm{Co}^{2+}$ than the studies in which a stimulatory effect on differentiation and resorptive activity of osteoclasts was observed. Nevertheless, differences in experimental set up (e.g. cell type) do not allow a direct comparison and therefore, an effect of $\mathrm{Co}^{2+}$ ions on osteoclast formation observed in our study cannot be fully excluded.

While low doses of $\mathrm{Co}^{2+}$ seem to stimulate osteoclast resorptive activity, there is no information on whether they play a role in actin ring formation. Substrate stiffness, on the other hand, has been suggested to have an influence on actin ring formation. In a study where the formation of podosomes by osteoclasts cultured on polyacrylamide and polydimethylsiloxane surfaces ranging in stiffness from $30 \mathrm{kPa}$ to around $1800 \mathrm{kPa}$ was investigated, it was shown that podosome belt formation 
was possible in this range of stiffness, as long as integrins were activated (e.g., by coating the substrate surfaces with either collagen or vitronectin). ${ }^{[27]}$ These findings suggested that actin ring formation can occur, to a certain extent, independently of substrate stiffness, if all other conditions (e.g. integrin activation) are met. In another study, it was shown that osteoclasts were also able to form actin rings on collagencoated coverslips, but not on the surface of collagen gels.[28] This study suggested that the mechanical properties and/or the porous, mesh-like surface of the gel impeded actin ring formation.

In our study, we have shown that biomineralized collagen, with stiffness of around $1000 \mathrm{kPa}$, did not allow actin ring formation on its surface. Since biomineralized collagen contains ligands for $\beta_{1}$ integrin activation (but not ligands for the vitronectin receptor), and stiffness within the range that allows actin ring formation, it is suggested that the porous structure was the inhibiting factor for actin ring formation. However, when stiffness was increased up to $10 \mathrm{MPa}$ by coating the membrane with $\mathrm{CoCaP}$, actin rings were sporadically observed, which suggests that the inhibiting effect of the fibrillar surface on actin ring formation can be at least partially overcome by increasing the substrate stiffness.

\subsection{Presence of Co and increased stiffness stimulate limited resorption of the coated biomineralized collagen membrane}

Resorption of the biomineralized membranes was investigated by quantification of $\mathrm{Ca}^{2+}$ in cell culture medium by ICP-MS, and also by observation of the membranes by SEM, after cell removal. Higher $\mathrm{Ca}^{2+}$ concentration was detected in culture medium from $\mathrm{CoCaP}$-coated membranes at 2, 9 and 16 days. While it is unlikely that resorption occurred as early as on day 2 , as osteoclasts were not observed on the coated membrane even after 7 days (Figure 2), it is plausible that the higher $\mathrm{Ca}^{2+}$ content in differentiation medium at 9 and 16 days was due to resorption of the substrate. However, these results must be interpreted with caution, due to the fact that only a few resorbing osteoclasts were observed and that this technique is not optimal for determining small differences in osteoclastic resorption.

There were no signs of resorption on the uncoated membranes (Figure 5A, 5C), but resorption lacunae were observed on the CoCaP-coated membrane (Figure 5B, 5D). These were of the same size as the actin rings observed by confocal laser microscopy. The observation of actin rings by fluorescence microscopy, together with SEM 
observations of resorption lacunae, and increased $\mathrm{Ca}^{2+}$ in cell culture medium after 9 and 16 days all point towards events of osteoclast resorption taking place on the $\mathrm{CoCaP}$-coated biomineralized membrane, although to a limited extent. Another interesting observation was that all resorption lacunae observed using SEM had a pit-like structure, while no trench-like resorption lacunae were observed.

In the previous section, we discussed the influence of substrate stiffness on actin ring formation by osteoclasts on different types of substrate. To our knowledge, no studies exist that investigated the effect of stiffness on osteoclastic resorption, especially in the range of $\mathrm{kPa}$ to $\mathrm{MPa}$, which would be useful to compare to our own data. From various resorption studies on bone, dentine, or CaP ceramics, it is evident that in-vitro resorption on surfaces with higher stiffness is common. ${ }^{[29-31]}$ We have also performed differentiation and resorption experiments on porous $\beta$-TCP ceramic discs to confirm that the differentiated osteoclasts were able to resorb this substrate (Supplementary Figure 1). The stiffness of bone, dentine and CaP ceramics is in the GPa range, thus orders of magnitude higher than the biomineralized collage substrates used in our study. Although resorption studies on polymer-CaP composites exist, ${ }^{[32-34]}$ and these materials can have lower stiffness, the resorption results in these studies are commonly not discussed in the context of the mechanical properties of the substrate.

Taken together, the results of this study showed that coating a biomineralized collagen membrane with a layer of Co-containing $\mathrm{CaP}$ had a twofold effect on the membrane properties, i.e. the stiffness of the membrane was increased by an order of magnitude, to $10 \mathrm{MPa}$, and Co was added to the substrate (Figure 1D, 1F). Additional CaP (Figure 1C) that was added by the coating process was involved in both effects by, on the one hand stiffening the membrane and on the other acting as a carrier of Co. Interestingly, no obvious effects of the additional coating was observed on the fibril thickness and hence the porosity of the membrane (Figure 1A, 1B). Osteoclasts, differentiated from human peripheral blood monocytes, were able to form on the coated membranes, and were also capable of resorbing it, albeit to a limited extent. This is supported by the observation of actin ring formation using fluorescence microscopy (Figure 2F), as well as by the observation of pit-like formations on the membranes, using SEM after cell removal (Figure 5B, 5D). The higher concentration of $\mathrm{Ca}^{2+}$ in cell culture medium of differentiated cells at days 9 
and 16 also support the few, and sporadic resorption events observed (Figure 4B). Although the effect of $\mathrm{CO}^{2+}$ ions, which were present at a low concentration (Figure 1D), cannot be excluded, we hypothesize that the main contributor to the ability of osteoclasts to resorb the coated membrane was the increase in membrane stiffness (though still significantly lower than that of bone or dentin slices), that allowed sealing of a compartment for resorption, partly overcoming the resorptioninhibiting effect of the large pores.

The main aim of this study was to modify the biomineralized collagen membrane in such a way that it can be resorbed by osteoclasts by adding $\mathrm{Co}^{2+}$ ions to the material. As discussed above, since the method we used to achieve this changed more than only the chemical properties of the membrane, the limitation of this study is that we cannot conclude which material property was responsible for the observed biological response. To this end, additional experiments separating the effects of stiffness and chemistry are required.

\section{Conclusion}

In conclusion, the results of this study showed that by a relatively simple modification of a biomineralized collagen membrane, resulting in increased stiffness and addition of a bioactive ion, it was possible to trigger the osteoclastic resorption of the membrane, though to a limited extent. The results of this study provide some directions for continued improvement of biomineralized collagen materials for application as bone graft substitutes. First, it is suggested that increase in collagen membrane stiffness enhances osteoclastic resorption. This can be achieved by compacting the collagen before intrafibrillar mineralization, or by increasing the amount of extrafibrillar mineral surrounding the already-mineralized fibers. The latter would have the same effect as reducing the pore size by compacting, which is an increase in stiffness, likely resulting in a surface more amenable to osteoclastic resorption. Secondly, the method of introducing bioactive ions to the membranes by post modification as described here offers an attractive alternative to incorporation during the intrafibrillar mineralization with the PILP method, which is complex, and should be explored more extensively for different types of ions. 


\section{Data availability statement}

The dataset supporting the conclusions of this article is available in the Dataverse repository, https://doi.org/10.34894/JUS4U6.

\section{Funding}

This research has been made possible with the support of the Dutch Province of Limburg (LINK project). PH gratefully acknowledges the Gravitation Program 'Materials-Driven Regeneration', funded by the Netherlands Organisation for Scientific Research (NWO).

\section{Acknowledgements}

The authors would like to thank Myraise van Stijn and Enabling Technologies/DSM for assistance with SEM-EDS analyses.

\section{Conflict of interest}

The authors confirm that there are no known conflicts of interest associated with this publication and there has been no significant financial support for this work that could have influenced its outcome. 


\section{Supplementary Material}
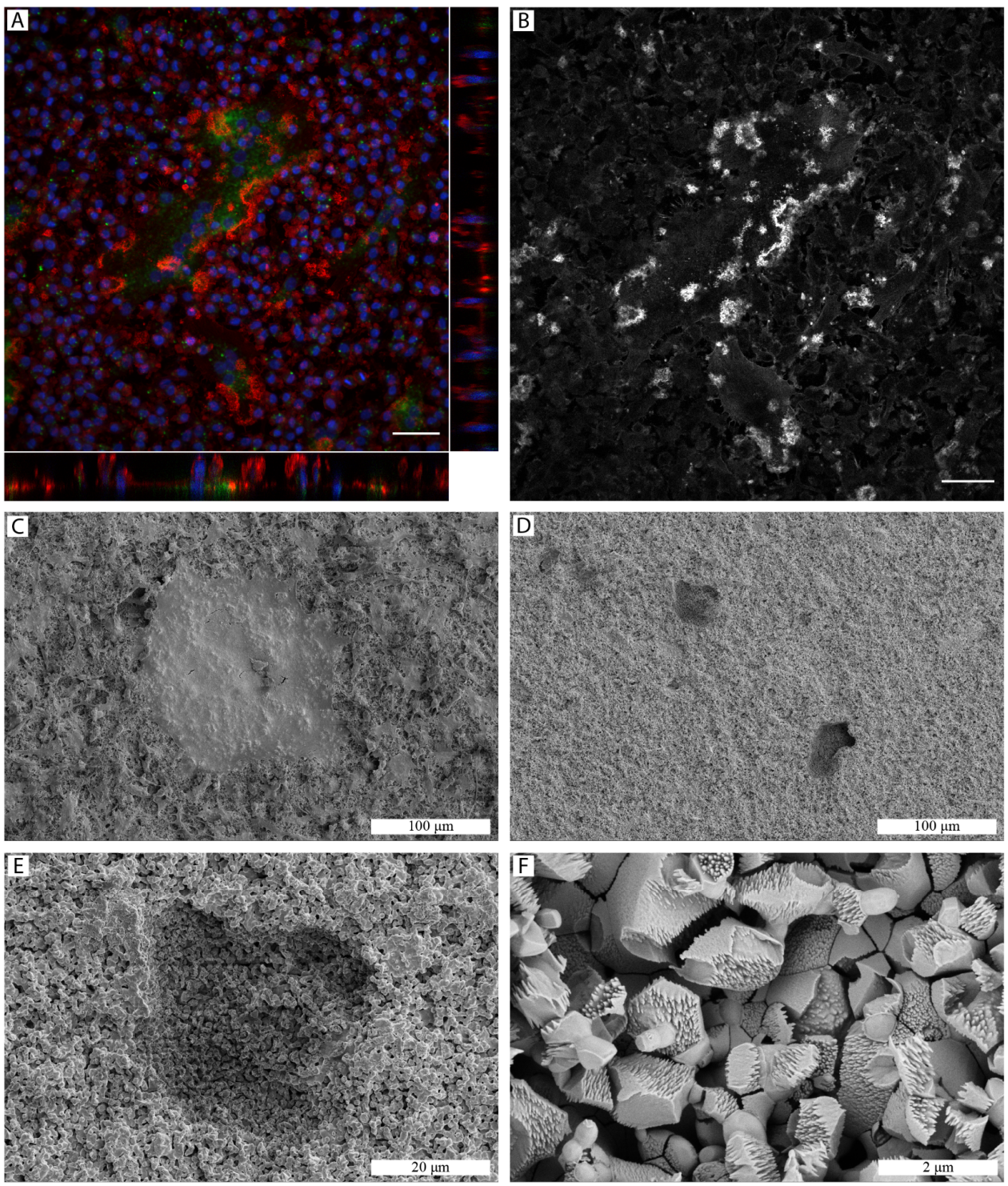

Supplementary Figure 1. Confocal laser microscopy (A, B) and SEM (C-F) images of osteoclasts culture on $\beta$-TCP discs, after 21-day cell culture. Laser confocal microscopy image (A) showed actin structures resembling resorption rings in close contact with the substrate surface (scale bar $50 \mu \mathrm{m}$ ). These are more clearly visible in the grayscale actin image (B), taken from a $\mathrm{Z}$ position close to the surface of the ceramic disc. SEM image of a large cell (C), presumably an osteoclast. Morphology of resorption pit (C-F) at different magnifications. The interior of the pit $(\mathrm{F})$ showed $\beta$-TCP grains with etched surface 


\section{References}

[1] M. J. Olszta, X. Cheng, S. S. Jee, R. Kumar, Y. Y. Kim, M. J. Kaufman, E. P. Douglas, L.

B. Gower, Mater. Sci. Eng. R Reports 2007, 58, 77.

[2] U. G. K. Wegst, H. Bai, E. Saiz, A. P. Tomsia, R. O. Ritchie, Nat. Mater. 2015, 14, 23.

[3] O. A. Tertuliano, J. R. Greer, Nat. Mater. 2016, 15, 1195.

[4] T. Kurien, R. G. Pearson, B. E. Scammell, Bone Jt. J. 2013, 95 B, 583.

[5] T. Rolvien, M. Barbeck, S. Wenisch, M. Amling, M. Krause, Int. J. Mol. Sci. 2018, 19, DOI 10.3390/ijms19102893.

[6] Z. Zhang, J. T. Egaña, A. K. Reckhenrich, T. L. Schenck, J. A. Lohmeyer, J. T. Schantz, H. Machens, A. F. Schilling, Acta Biomater. 2012, 8, 13.

[7] K. P. McHugh, K. Hodivala-Dilke, M. H. Zheng, N. Namba, L. Jonathan, D. Novack, X. Feng, F. P. Ross, R. O. Hynes, S. L. Teitelbaum, J. Clin. Invest. 2000, 105, 433.

[8] I. Nakamura, N. Takahashi, E. Jimi, N. Udagawa, T. Suda, Mod. Rheumatol. 2012, 22, 167.

[9] S. Patntirapong, P. Habibovic, P. V. Hauschka, Biomaterials 2009, 30, 548.

[10] F. Nudelman, P. H. H. Bomans, A. George, G. de With, N. A. J. M. Sommerdijk, Faraday Discuss. 2012, 159, 357.

[11] L. B. Gower, in Biominer. Biomater. Fundam. Appl., 2015.

[12] Z. Tahmasebi Birgani, A. Malhotra, L. Yang, B. Harink, P. Habibovic, in (Ed: P. B. T.C. B. I. I. Ducheyne), Elsevier, Oxford, 2017, pp. 406-427.

[13] N. L. Davison, X. Luo, T. Schoenmaker, V. Everts, H. Yuan, F. Barrère-de Groot, J. D. de Bruijn, Eur. Cells Mater. 2014, 27, 281.

[14] D. de Melo Pereira, M. Eischen-Loges, Z. T. Birgani, P. Habibovic, Front. Bioeng. Biotechnol. 2020, 8, 1248.

[15] Y. Li, T. T. Thula, S. Jee, S. L. Perkins, C. Aparicio, E. P. Douglas, L. B. Gower, Biomacromolecules 2011, 13, 49.

[16] L. Filgueira, J. Histochem. Cytochem. 2004, 52, 411.

[17] S. M. Maria, C. Prukner, Z. Sheikh, F. Mueller, J. E. Barralet, S. V. Komarova, J. Biomed. Mater. Res. - Part B Appl. Biomater. 2014, 102, 903.

[18] L. Yang, S. Perez-Amodio, F. Y. F. Barrère-de Groot, V. Everts, C. A. van Blitterswijk, P. Habibovic, Biomaterials 2010, 31, 2976.

[19] A. A. Al-Munajjed, N. A. Plunkett, J. P. Gleeson, T. Weber, C. Jungreuthmayer, T. Levingstone, J. Hammer, F. J. O’Brien, J. Biomed. Mater. Res. - Part B Appl. Biomater. 2009, 90 B, 584.

[20] D. Kim, B. Lee, S. Thomopoulos, Y.-S. Jun, Cryst. Growth Des. 2016, 16, 5359.

[21] H. S. Yang, W.-G. La, J. Park, C.-S. Kim, G.-I. Im, B.-S. Kim, J. Biomater. Sci. Polym. Ed. 2012, 23, 1659.

[22] P. G. Koutsoukos, in (Ed: Z. Amjad), Springer US, Boston, MA, 1998, pp. 41-66.

[23] P. G. Koutsoukos, G. H. Nancollas, Colloids and Surfaces 1987, $28,95$.

[24] A. L. Boskey, J. Phys. Chem. 1989, 93, 1628.

[25] A. Bernhardt, K. Koperski, M. Schumacher, M. Gelinsky, Eur. Cells Mater. 2017, 33, 28.

[26] R. E. Andrews, K. M. Shah, J. M. Wilkinson, A. Gartland, Bone 2011, 49, 717. 
[27] H. Touaitahuata, E. Planus, C. Albiges-Rizo, A. Blangy, G. Pawlak, Eur. J. Cell Biol. 2013, 92, 139.

[28] I. Nakamura, N. Takahashi, T. Sasaki, E. Jimi, T. Kurokawa, T. Suda, J. Bone Miner. Res. 1996, 11, 1873.

[29] J. Keller, S. Brink, B. Busse, A. F. Schilling, T. Schinke, M. Amling, T. Lange, PLoS One 2012, 7, DOI 10.1371/journal.pone.0046757.

[30] V. Perrotti, B. M. Nicholls, A. Piattelli, Clin. Oral Implants Res. 2009, 20, 17.

[31] T. Winkler, E. Hoenig, R. Gildenhaar, G. Berger, D. Fritsch, R. Janssen, M. M. Morlock, A. F. Schilling, Acta Biomater. 2010, 6, 4127.

[32] N. Rochet, T. Balaguer, F. Boukhechba, J. P. Laugier, D. Quincey, S. Goncalves, G. F. Carle, Biomaterials 2009, 30, 4260.

[33] V. Nicolin, G. Baldini, D. De Iaco, R. Bortul, G. Turco, S. L. Nori, Transl. Med. @ UniSa 2016, 14, 15.

[34] S. M. Cool, B. Kenny, A. Wu, V. Nurcombe, M. Trau, A. I. Cassady, L. Grøndahl, J. Biomed. Mater. Res. Part A 2006, 79, 963. 


\section{Chapter 5}

\section{Proliferation and osteogenic differentiation of hMSCs on biomineralized collagen}

Daniel de Melo Pereira, Maria Eischen-Loges, Zeinab Tahmasebi Birgani, Pamela Habibović

Published in Frontiers in Bioengineering and Biotechnology, 2020, 8:554565, 1-16 


\section{Abstract}

Biomineralized collagen with intrafibrillar calcium phosphate mineral provides an excellent mimic of the composition and structure of the extracellular matrix of bone, from nano- to micro scale. Scaffolds prepared from this material have the potential to become the next-generation of synthetic bone graft substitutes, as their unique properties make them closer to the native tissue than synthetic alternatives currently available to clinicians. To understand the interaction between biomineralized collagen and cells that are relevant in the context of bone regeneration, we studied the growth and osteogenic differentiation of bone marrow derived human mesenchymal stromal cells (hMSCs) cultured on biomineralized collagen membranes, and compared it to the cell behavior on collagen membranes without mineral. Cells proliferated normally on both biomimetic membranes, and were more triggered to differentiate toward the osteogenic lineage by the biomineralized collagen. This was shown by the elevated mRNA levels of RUNX2, SPP1, ENPP1 and OCN after 3 days of culture, and COL1A1 after 14 days of culture on mineralized collagen. The mRNA levels of the tested markers of osteogenesis were lower on collagen membranes without mineral, with the exception of OCN, which was more highly expressed on collagen than on biomineralized collagen membranes. Expression by hMSCs of OPG, a gene involved in inhibition of osteoclastogenesis, was higher on biomineralized collagen at day 3, while M-CSF, involved in osteoblast-osteoclast communication, was upregulated on both membranes at day 3 and 14 of culture. Alkaline phosphatase activity of hMSCs was high on both biomimetic membranes when compared with cells cultured on tissue culture plastic. Cell-induced mineralization was observed on collagen membranes, while the high mineral content of the biomineralized membranes prohibited a reliable analysis of cell-induced mineralization on these membranes.

In conclusion, we have identified that both collagen and biomineralized collagen support proliferation, osteogenic differentiation and mineralization of hMSCs, with biomineralized membranes having a more pronounced positive effect. These findings support the existing evidence that biomineralized collagen is a promising material in the field of bone regeneration. 


\section{Introduction}

In the field of bone repair and regeneration, there is a growing need for alternatives to autologous bone transplant as the standard of care. A biomaterial that is able to promote bone growth and regeneration of the injury site, at least as efficiently as autologous bone, is highly desirable. The two main advantages would be increased availability offered by a synthetic biomaterial, and elimination of complications associated with harvesting of bone tissue for transplantation.[1,2]

A majority of commonly used synthetic bone graft substitutes in the clinic are calcium phosphate $(\mathrm{CaP})$ ceramics, or composites of a $\mathrm{CaP}$ ceramic with an organic component, often collagen. ${ }^{[3-5]}$ Such composite biomaterials are similar in composition to the extracellular matrix (ECM) of bone, which consists of about 65 $w t \%$ inorganic matrix (hydroxyapatite - HA), $25 \mathrm{wt} \%$ organic matrix (mostly collagen type I) and $10 \%$ water. ${ }^{[6]}$ However, they do not completely replicate the organized hierarchical structure of bone, which, in addition to the composite nature, is known to contribute to the unique mechanical properties of bone. ${ }^{[7,8]}$ The structural organization of bone at different length scales comes as a natural source of inspiration for designing materials with potential to become next-generation synthetic bone graft substitutes.

Several attempts have been made to bridge the current gap between synthetic bone graft substitutes and native bone tissue, by replicating the organic-inorganic structure of bone from sub-micrometer to centimeter level.[9] The replication of the base unit of the bone ECM is of particular interest, as it potentially allows the bottom-up building of larger biomimetic constructs. The building block of bone ECM consists of collagen fibrils with high content $(\geq 65 \%(w / w))$ of intrafibrillar mineral, specifically nano HA crystals with their c-axis aligned along the collagen fibril length. ${ }^{6]}$ Intrafibrillary mineralized collagen materials can be prepared in vitro, ${ }^{[10]}$ via a mineralization method that typically involves one or more charged polymers. These polymers act in vitro as analogues of non-collagenous proteins present in the native ECM of bone. They effectively inhibit homogeneous crystallization, i.e., crystallization in solution, forming amorphous CaP-polymer complexes, and promoting mineralization primarily inside the ordered nanostructure of collagen type I fibers. ${ }^{[11-13]}$ 
The potential of biomineralized collagen as the basis for novel bone graft substitutes is evidenced by several studies that investigated this biomaterial in the context of induction of osteogenic differentiation in vitro and regeneration of bone defects in vivo. A few studies have looked at the extent of osteogenic differentiation of osteoblast-like cell lines, ${ }^{[14,15]}$ mouse, ${ }^{[16]}$ or rat MSCs,,$^{[17,18]}$ induced by biomaterials containing biomineralized collagen in their composition. With respect to the application of these materials in the context of bone regeneration, however, more relevant information can be obtained from studies with primary human cells which, though limited in number and using diverse experimental designs and cell types, all have the common denominator of having a scaffold composed of collagen with intrafibrillar mineral and studying the osteogenic differentiation of multipotent cells. HMSCs were embedded in a collagen gel that was biomineralized with an adapted PILP method, using milk osteopontin as process-directing agent to obtain intrafibrillar as well as extrafibrillar apatite mineral. Gene expression analysis showed that cells within mineralized gels had similar or higher mRNA expression of runt-related transcription factor 2 (RUNX-2), osteocalcin OCN, podoplanin (PDPN) and dentin matrix protein 1 (DMP1), than cells encapsulated in a nonmineralized gel, and moreover that OCN, PDPN and DMP1 were expressed at levels comparable to or higher than gel-encapsulated cells cultured with osteogenic induction medium. Protein quantification showed that the mineralized collagen gel induced cells to express a higher RANK-L to OPG ratio than either the unmineralized gel or the osteogenic induction medium, showing the potential for this material to modulate the communication between osteoblast and osteoclast precursors.[19]

In a study using periodontal ligament stem cells, increased expression of osteopontin, or secreted phosphoprotein 1 (SPP1), collagen type I (COL1A1), and bone morphogenetic protein 2 (BMP-2) was shown after 7 and 14 days of culture on collagen with intrafibrillar mineral versus unmineralized collagen and collagen with extrafibrillar mineral controls. There was also an increased production of mineral nodules. ${ }^{[20]}$

Umbilical cord-derived MSCs cultured on scaffolds made of biomineralized collagen showed alkaline phosphatase (ALP) activity similar to cells cultured in the established osteogenic differentiation medium on tissue culture plastic. The 
scaffolds were implanted in a rabbit femur defect model (Ø8 × $6 \mathrm{~mm}$ ), showing almost complete healing after 12 weeks. ${ }^{[21]}$

A scaffold composed of nano-HA, collagen and poly-L-lactic acid induced osteogenic differentiation of hMSCs, with upregulation of BMP-2, COL1A1 and Cathepsin-K (CTSK), shown by microarray analysis. ${ }^{[22]}$ This biomaterial was also used to repair calcaneal fractures in human patients, where a comparison with autologous bone graft showed no difference in clinical outcome. Seven out of 24 patients had harvest-related complications after 12 months, which is avoidable with the use of synthetic a bone graft substitute. ${ }^{[23]}$ However, it is not entirely clear whether intrafibrillar mineral was present in this material.[24]

While these few studies show the potential for biomineralized collagen to induce osteogenic differentiation, as well as promising bone defect healing capacity, knowledge about if and how biomineralized collagen triggers osteogenic differentiation is by no means complete. More knowledge of differentiation processes triggered by this biomimetic material and how they relate to osteoinduction in vivo is needed to ultimately develop more effective synthetic bone graft substitutes.

In this study, we applied a recognized method for producing biomineralized collagen membranes, and used this material to study the osteogenic differentiation of bone marrow-derived hMSCs. We looked into mRNA expression of early and late markers of osteogenesis and as well as markers involved in osteoblast-osteoclast communication. Furthermore, we characterized the cells regarding their ALP activity and capacity for mineralization.

\section{Materials and methods}

\subsection{Materials}

PureCol@ collagen type I solution (col-I, $3 \mathrm{mg} / \mathrm{mL}$, 97\% bovine dermal type I atelocollagen) was purchased from Advanced BioMatrix (California, USA, cat\# 5005). Poly-L-aspartic acid sodium salt ( $\mathrm{pAsp}, \mathrm{Mw}=27 \mathrm{kDa}$ ) was purchased from Alamanda Polymers (Alabama, USA, cat\# 000-D200). 1-Ethyl-3-[3dimethylaminopropyl] carbodiimide hydrochloride (EDC), Nhydroxysulfosuccinimide (sulfo-NHS), calcium chloride dihydrate, potassium 
phosphate dibasic, and all other chemicals were purchased from Sigma-Aldrich (Missouri, USA).

\subsection{Preparation of collagen membranes}

Dense collagen films were prepared in a sterile environment according to the process described by Li et al. (2011), with some modifications. Briefly, the collagen solution, $10 \mathrm{x}$ phosphate buffered saline (PBS) and $0.1 \mathrm{M} \mathrm{NaOH}$ were mixed in a volume fraction of, respectively, 0.706, 0.176, and 0.118, yielding a final collagen concentration of $2.1 \mathrm{mg} / \mathrm{mL}$. Gels of $2 \mathrm{~mL}$ were formed in up-facing $5 \mathrm{~mL}$ syringes with the tip cut off (Sigma-Aldrich, cat\# Z248010), at $37{ }^{\circ} \mathrm{C}$ for $24 \mathrm{~h}$. The syringes containing the gels were inverted on top of nylon meshes of $40 \mu \mathrm{m}$ pore size (Fischer Scientific, Massachusetts, USA, cat\# 11587522) and left at $37{ }^{\circ} \mathrm{C}$ for $48 \mathrm{~h}$ to make the gels loose water under their own weight. After this compression step, syringes were removed, collagen membranes were washed with PBS and cross-linked with $50 \mathrm{mM}$ EDC, $25 \mathrm{mM}$ sulfo-NHS in $50 \mathrm{mM}$ 4-Morpholineethanesulfonic acid (MES) buffer $(\mathrm{pH}=7.0)$, overnight at room temperature. The following day, gels were washed with $\mathrm{PBS}$ and incubated with $0.1 \mathrm{M} \mathrm{Na}_{2} \mathrm{HPO}_{4}$ and $2 \mathrm{M} \mathrm{NaCl}$ for $2 \mathrm{~h}$, to quench the remaining activated carboxylic acid residues. The membranes were washed three times with PBS for $2 \mathrm{~h}$. Membranes were then mineralized or kept in PBS at $4{ }^{\circ} \mathrm{C}$ for maximum 1 week.

\subsection{Mineralization of collagen membranes}

Mineralization solution was prepared according to the polymer-induced liquid precursor (PILP) method.[10] Briefly, stock solutions of calcium $\left(9 \mathrm{mM} \mathrm{CaCl}_{2}\right)$ and phosphate ( $4.2 \mathrm{mM} \mathrm{K}_{2} \mathrm{HPO}_{4}$ ) were prepared in a buffer with $50 \mathrm{mM}$ TRIS base, 150 $\mathrm{mM} \mathrm{NaCl}$, with a $\mathrm{pH}$ of 7.8 at room temperature. Prior to incubation, pAsp was added to the calcium solution, mixed and let rest for $5 \mathrm{~min}$, followed by addition of the same volume of phosphate precursor solution. The final concentration of $\mathrm{pAsp}$ was $100 \mu \mathrm{g} / \mathrm{mL}$. After mixing the two precursor solutions, the col-I membranes were added ( $40 \mathrm{~mL}$ of solution was used per membrane) and incubated in a water bath at $37^{\circ} \mathrm{C}$ for 7 days. Before cell culture, membranes were punched with a $10 \mathrm{~mm}$ metal puncher for culture in 48-well plates. Collagen or biomineralized collagen membranes were washed with PBS and incubated in cell culture medium for 3-4 h, prior to cell culture. All steps were performed in a sterile environment. 


\subsection{Cell culture}

Human mesenchymal stromal cells were isolated from bone marrow aspirates ${ }^{[2,26]}$ obtained from one donor, who has given written informed consent. After isolation, the cells were seeded at a density of $1500 \mathrm{cells} / \mathrm{cm}^{2}$ in tissue culture T-flasks, and expanded in growth medium (GM), consisting of $\alpha$-MEM without nucleotides and with Glutamax ${ }^{\mathrm{TM}}$ (ThermoFisher Scientific, cat\# 32561), supplemented with $10 \mathrm{v} / \mathrm{v} \%$ fetal bovine serum (Sigma-Aldrich, cat\# F7524, batch\# BCBT6987), and $20 \mathrm{mM}$ ascorbic acid (Sigma-Aldrich, cat\# A8960). Osteogenic differentiation medium (OM) was prepared by supplementing GM with $100 \mathrm{nM}$ dexamethasone (Sigma-Aldrich, cat\# D8893). Mineralization medium (MM) was prepared by supplementing OM with $10 \mathrm{mM} \beta$-glyrecophosphate (Sigma-Aldrich cat\# 50020). Cells were cultured in standard conditions of $37^{\circ} \mathrm{C}$ in a humidified atmosphere with $5 \% \mathrm{CO}_{2}$, and medium was replaced every 2 or 3 days. Upon reaching $70 \%-80 \%$ confluence, the cell layer was washed with warm PBS and detached using a $0.05 \%$ trypsin-EDTA solution (Thermo Fisher Scientific, cat\# 253000) until detachment was visible under a light microscope, up to a maximum of $5 \mathrm{~min}$. Trypsin was neutralized with addition of GM, the cell suspension was centrifuged at $300 \mathrm{rcf}$ for $5 \mathrm{~min}$ and re-suspended in GM. Cells were counted with a haemocytometer and dilution of the cell suspension was made according to the seeding densities required.

For quantification of DNA and ALP activity, which were performed on the same set of samples, cells were seeded at a density of $10.000 \mathrm{cell} / \mathrm{cm}^{2}$ in 48 -well plates on collagen or biomineralized collagen, with tissue culture plastic (TCP) as a control. Cells on TCP were cultured in GM or OM, while on both types of membranes, the culture was performed in GM only. There were six replicates per condition. At each time point $(3,7,14$, and 21 days), medium was aspirated and samples washed with warm PBS. All PBS was removed and the plate was kept at $-80{ }^{\circ} \mathrm{C}$ until further analysis.

The same seeding and sample handling was performed for the quantitative real-time polymerase chain reaction (qRT-PCR) experiment, at 3, 7, and 14 days, also with 6 replicates per condition.

\subsection{DNA quantification}

Total content of nucleic acid, i.e., cell DNA, per sample was quantified with the Cyquant $^{\mathrm{TM}}$ kit (Thermo Fisher Scientific, cat\# C7026) following the manufacturer's instructions. Briefly, frozen plates were thawed and collagen and mineralized 
collagen membranes were transferred to new plates. Lysis buffer from the kit was added to each well $(300 \mu \mathrm{L})$ and two more freeze-thaw cycles were done. Subsequently, samples were placed on ice in a ultrasonic water bath for $30 \mathrm{~min}$. Cell lysates were collected and transferred to micro centrifuge tubes, spun down for $10 \mathrm{~s}$ and $100 \mu \mathrm{L}$ of the supernatant transferred to clear bottom black 96-well plates, with technical duplicates. The dye-containing buffer was added $(2 x$ concentrated, $100 \mu \mathrm{L})$ and plates were incubated at room temperature for $5 \mathrm{~min}$ in the dark. Fluorescence measurements were made with a CLARIOstar Plus microplate reader (BGM Labtech, Germany) with excitation and emission wavelength of $485 \pm 10$ and $530 \pm$ $10 \mathrm{~nm}$, respectively. A standard curve of known cell numbers versus fluorescence intensity was prepared from the same cell suspension used in the experiment, i.e., $11 \mathrm{k}, 22 \mathrm{k}, 33 \mathrm{k}, 44 \mathrm{k}$, and 110k cells in 48-well plate, frozen after $6 \mathrm{~h}$ in culture. Total cell number per condition was calculated by subtracting the blank fluorescence reading, i.e., dye-containing lysis buffer, from each measurement, and converting fluorescence to cell number with the standard curve. The results are presented as mean and standard deviation of the biological replicates.

\section{6. $\mathrm{qRT}-\mathrm{PCR}$}

Samples were collected in TRIzol Reagent (Thermo Fisher Scientific). Extraction of RNA was performed using the phenol-chloroform method and purification was done using the RNeasy Minikit (QUIAGEN, Germany), in accordance with the manufacturer's recommendations. The extracted RNA of biological replicates were pooled in groups of 2, in order to increase the RNA quantity in each sample, resulting in 3 replicates per condition. RNA purity and quantity were determined using a BioDrop $\mu$ LITE instrument (BioDrop, UK). For each sample, 250 ng of RNA were reverse transcribed into cDNA using iScript cDNA Synthesis Kit (Bio-Rad, California, USA) following manufacturer's instructions. Amplification of cDNA (20 ng) by qRT-PCR was performed on a CFX96 Real-Time PCR Detection System (BioRad) using the iQ SYBR Green Supermix for qPCR (Bio-Rad). Transcription levels of osteogenic biomarkers including runt-related transcription factor 2 (RUNX2), SPP1, ALP, COL1A1, osteocalcin (OCN), Ectonucleotide Pyrophosphatase/Phosphodiesterase 1 (ENPP1) as well as osteoclast modulatory biomarkers including receptor activator of nuclear factor kappa-B ligand (RANKL), osteoprotegerin (OPG) and macrophage colony-stimulating factor (MCSF), were 
determined. Primer sequences for each marker can be found in Table 1. Fold expression values were determined using $\Delta \Delta \mathrm{Ct}$ method after normalizing each target gene with respect to the housekeeping gene (GAPDH) and to the expression of the target gene in hMSCs at day 3 on culture plates in GM.

Table 1. Primer sequences for qRT-PCT

\begin{tabular}{lll}
\hline \multicolumn{1}{c}{ Gene } & \multicolumn{1}{c}{ Forward $\left(5^{\prime}-3^{\prime}\right)$} & \multicolumn{1}{c}{ Reverse $\left(5^{\prime}-3^{\prime}\right)$} \\
\hline RUNX2 & CCGCCTCAGTGATTTAGGGC & GGGTCTGTAATCTGACTCTGTCC \\
SPP1 & GGTGATGTCCTCGTCTGTA & CCAAGTAAGTCCAACGAAAG \\
ALP & ACAAGCACTCCCACTTCATC & TTCAGCTCGTACTGCATGTC \\
COL1A1 & GAGGGCCAAGACGAAGACATC & CAGATCACGTCATCGCACAAC \\
OCN & TGAGAGCCCTCACACTCCTC & CGCCTGGGTCTCTTCACTAC \\
ENPP1 & CAAAGGTCGCTGTTTCGAGAG & TGCACGTCTCCTGGTAATCTAAA \\
RANKL & CAACATATCGTTGGATCACAGCA & GACAGACTCACTTTATGGGAACC \\
OPG & CACAAATTGCAGTGTCTTTGGTC & TCTGCGTTTACTTTGGTGCCA \\
MCSF & AGACCTCGTGCCAAATTACATT & AGGTGTCTCATAGAAAGTTCGGA \\
\hline
\end{tabular}

\subsection{Quantification of ALP activity}

Alkaline phosphatase activity was quantified with the CDPStar ${ }^{\circledR}$ reagent (SigmaAldrich, cat\# GERPN3682). $10 \mu \mathrm{l}$ of cell lysate supernatant (as described for quantification of total cell number) were transferred, with technical duplicates, to a 96-well plate. $40 \mu \mathrm{L}$ of CDPStar reagent was added to each well, followed by $30 \mathrm{~min}$ incubation at room temperature in dark. Luminescence intensity was read with a CLARIOstar Plus microplate reader. The results were normalized to the cell number, and are presented as mean and standard deviation of the biological replicates.

\subsection{Scanning electron microscopy (SEM) and energy-dispersive X-ray} spectroscopy (EDS)

The morphology and chemical composition of the membranes were characterized using an SEM coupled with an EDS detector. Collagen and biomineralized collagen membranes were dehydrated using a sequence of, first, mixtures of PBS and ethanol (70:30, 60:40 and 50:50) for $15 \mathrm{~min}$ each; and second, mixtures of distilled water and ethanol (40:60, 30:70, 20:80, 10:90, and 100\% ethanol) for 15 min each. This was followed with a $30 \mathrm{~min}$ incubation in a 50:50 mixture of ethanol and hexadimethylsiloxane (HDMS), followed by $30 \mathrm{~min}$ of incubation in HDMS. The last step consisted of removing the HDMS and leaving the membranes to dry overnight. 
Dried membranes were glued to aluminum stubs with carbon tape and silver paint. Samples were sputter coated with a $2 \mathrm{~nm}$ iridium layer for increased conductivity using a Q150TES sputter coater (Quorum, UK).

For observation of cell-induced mineralization, membranes with cells cultured on them were washed with warm PBS following cell culture medium aspiration, and fixed with warm $4 \%$ formaldehyde in PBS, at room temperature for 15 min. After washing with PBS, the samples were dehydrated and prepared for SEM as described above.

Samples were imaged with a TENEO electron microscope (FEI, Oregon, USA) operating in Optiplan mode at $2-5 \mathrm{kV}$ and $2-10 \mathrm{~mm}$ working distance, using the $\mathrm{T} 1$ in-column, ETD or back-scatter detectors. For EDS analysis, samples were imaged with a VERSA electron microscope (FEI) equipped with an EDS detector (EDAX, New Jersey, USA), operating at $10 \mathrm{kV}$ and $10 \mathrm{~mm}$ working distance.

\subsection{Statistical analysis}

Statistical testing of mean differences was performed in GraphPad Prism (version 8.3) using two-way analysis of variance (ANOVA) of independent samples, looking at differences between conditions within each timepoint. A Bonferroni post hoc test was used to correct for multiple comparisons (one family for all comparisons). Mean differences were considered statistically significant for $\mathrm{p}$-value $<0.05$. Data in all figures is presented as mean and standard deviation, unless otherwise specified, and significance is denoted as $\left.\left.\left.\left(^{*}\right) \mathrm{p}<0.05,{ }^{(* *}\right) \mathrm{p}<0.01,{ }^{* * *}\right) \mathrm{p}<0.001,{ }^{* * * *}\right) \mathrm{p}<0.0001$. 


\section{Results}

\subsection{Characterization of collagen and biomineralized collagen membranes}

The morphology and elemental composition of both biomimetic membranes was analyzed by SEM-EDS, and the results are displayed in Figure 1. The membranes as used for cell culture are shown in Figure 1A.

The collagen membranes were compact films consisting of randomly oriented collagen fibers, with even surface morphology exemplified by the images in Figure $1 \mathrm{~B}$ and 1C. Fibers of the collagen matrix had an average diameter of $60 \pm 13 \mathrm{~nm}$ and displayed a banding pattern along their length (Figure 1D). The fibrous structure remained after the mineralization process, although the fibers were wider, $201 \pm 56$ $\mathrm{nm}$ (Figure 1E), and the banding pattern was no longer visible. Elemental analysis of both membranes showed that calcium and phosphorus was only present in biomineralized collagen membranes (Figure 1D, E).

\subsection{Effect of collagen and biomineralized collagen on proliferation of hMSCs}

To explore the effect of collagen and mineralized collagen on the proliferation of hMSCs, using TCP as a control, DNA content of the cells on membranes was quantified and converted to cell number (Figure 2). Cells cultured on TCP in GM exhibited a steady increase in number until day 14 . Beyond this time point, the cells were detached from the TCP due to overgrowth of the cell monolayer in the culture well, and hence, no measurement could be done for GM. hMSCs cultured in OM were proliferating at a lower rate than in GM at days 7 and 14, and no further growth in OM was observed between day 14 and 21. Cells cultured on collagen membranes showed a lower proliferation than on TCP in GM at days 3 and 7. A boost in proliferation was observed between day 7 and 14 on collagen membranes where cells reached the same cell number as on TCP in GM. No further increase in cell number was observed on collagen membranes at day 21. hMSCs cultured on mineralized collagen had a lower cell number than cells on TCP in GM at days 3, 7, and 14. No further growth was observed at day 21. On both collagen and mineralized collagen, cells followed a proliferation pattern similar to that on TCP in OM until day 7 of culture. At day 14 and 21, proliferation was increased on collagen membranes whereas cells on mineralized collagen maintained a proliferation similar to the cells cultured on TCP in OM. Taken together, both collagen and mineralized 
collagen supported proliferation of hMSCs, with, at the later time points of 14 and 21 days, a higher cell number on collagen than on mineralized collagen.

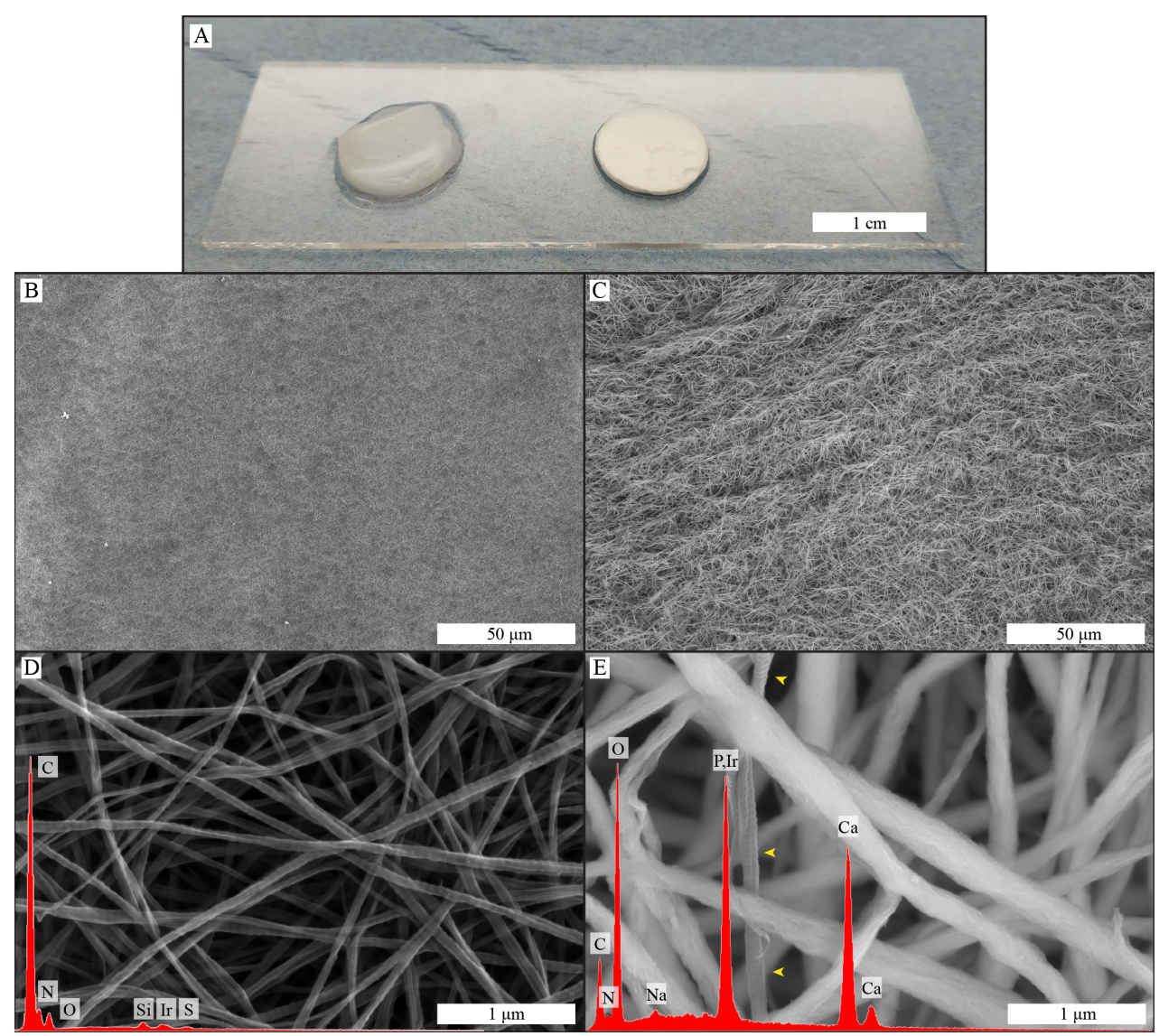

Figure 1. Macroscopic image (A) of collagen and biomineralized collagen as used for cell culture experiments; surface structure and elemental composition of collagen (B,D) and biomineralized collagen (C,E) membranes, obtained by SEM-EDS. Low magnifications images of the surface of collagen (B) and biomineralized collagen (C). High magnification image of collagen membrane with corresponding EDS spectrum (D), collagen banding is visible and there is no calcium or phosphorus signal. High magnification image of biomineralized collagen and corresponding EDS spectrum (E), where thicker fibers are visible, and no banding is discernible, expect in one fiber that is not mineralized (yellow arrowheads). Both types of membrane show fibrillar structure. 


\subsection{Osteogenic gene expression of hMSCs on biomimetic membranes}

Osteogenic differentiation of hMSCs was analyzed by quantifying the expression of osteogenic biomarkers at the mRNA level via qRT-qPCR (Figure 3). The selected markers were RUNX2, a transcription factor associated with activation of osteogenic genes $^{[27]}$ and SPP1, a non-collagenous protein with roles in cell attachment and matrix mineralization. ${ }^{[28]}$ In addition, markers for two matrix proteins were assessed, COL1A1, the main constituent of the organic part of the matrix ${ }^{[29]}$ and $\mathrm{OCN}$, which is involved in matrix mineralization by binding hydroxyapatite, ${ }^{[30]}$ as well as two other markers involved in the regulation of inorganic phosphate, ALP and ENPP1.[11,32]

RUNX2, an early transcription factor, ${ }^{[27]}$ was significantly upregulated at day 3 on collagen (1.6x) as well as on mineralized collagen (2.5x), compared with TCP. After 7 days of culture, a similar trend was observed, where a higher expression on both materials was detected as compared to the control, i.e., TCP, with the highest expression on biomineralized collagen. At day 14, when cells on both types of biomimetic membrane showed higher expression levels than the control, i.e. cells cultured on TCP, RUNX-2 expression on biomineralized collagen was significantly upregulated by 3.1x. When comparing collagen and mineralized collagen, a trend of a higher RUNX2 expression on mineralized collagen was observed at each time point.

A significant upregulation of SPP1 on biomimetic membranes as compared to TCP was detected at day 3 , with a $4 x$ increase on collagen and the highest increase of $18 x$ on mineralized collagen, following a behavior similar to RUNX2. After 7 days, no differences in expression of SPP1 were observed between conditions and, after 14 days, a slight decrease in expression relative to the control was observed on collagen, without statistical significance, whereas cells on mineralized collagen showed similar expression levels as the control. A decrease in expression over time was observed on mineralized collagen, while expression for TCP slightly increased from day 3 to 7 and were maintained at day 14 . The expression overtime on collagen was unchanged at 3 and 7 days and showed a slight decrease at 14 days.

Expression of ALP, which plays a role in matrix mineralization, ${ }^{[31,32]}$ was slightly downregulated early, at day 3 , on collagen relative to the control, as well as on mineralized collagen with a $2 x$ decrease. Comparable expression levels between all 
conditions were observed at day 7. After 14 days, the expression on collagen and mineralized collagen was increased as compared to TCP, with a $2.7 x$ statistically significant increase on mineralized collagen. ALP expression was downregulated in the TCP control overtime but collagen and mineralized collagen maintained the same level overtime.

Expression of ENPP1, an enzyme that regulates bone mineralization [31,32], was evaluated. Both ENPP1 and ALP are involved in maintaining bone mineralization in equilibrium. Bone mineralization relies on the availability of inorganic phosphate (Pi), which together with calcium crystallizes to form HA. ALP hydrolyses inorganic pyrophosphate (PPi) to generate $\mathrm{Pi}$, which promotes mineralization, and ENPP1 generates $\mathrm{PPi}$, which antagonizes mineralization. A statistically significant upregulation of ENPP1 was observed on both collagen (3x) and mineralized collagen (4.6x) relative to the control, at day 3. A significant increase of 1.6x was observed on mineralized collagen as compared to collagen. A pronounced decrease was observed at day 7 on collagen and mineralized collagen, as well as a smaller decrease on TCP. This trend was maintained after 14 days with a slight increase of expression on biomineralized collagen, without statistical significance.

OCN expression was significantly upregulated on collagen, being $3 x$ higher than on mineralized collagen and $8 \mathrm{x}$ higher as compared to TCP. The expression on mineralized was also significantly increased $(2.4 \mathrm{x})$ as compared to the control. This trend of highest expression on collagen, followed by mineralized collagen and the lowest being on TCP, was maintained at days 7 and 14, although the difference of expression between collagen and mineralized was not as pronounced as at the earliest time point. A decrease of OCN expression was observed over time for all substrates.

COL1A1, the main constituent of the organic matrix and therefore an indicator of ECM deposition, ${ }^{[29]}$ was significantly downregulated on collagen at day 3 , by half relative to the control, expression on mineralized collagen was similar as on TCP. No differences between conditions was observed at day 7. At day 14 on mineralized collagen, COL1A1 expression increased significantly by $5 x$ relative to TCP and by $3.4 \mathrm{x}$ relative to collagen. A decreasing trend over time was observed on TCP. 

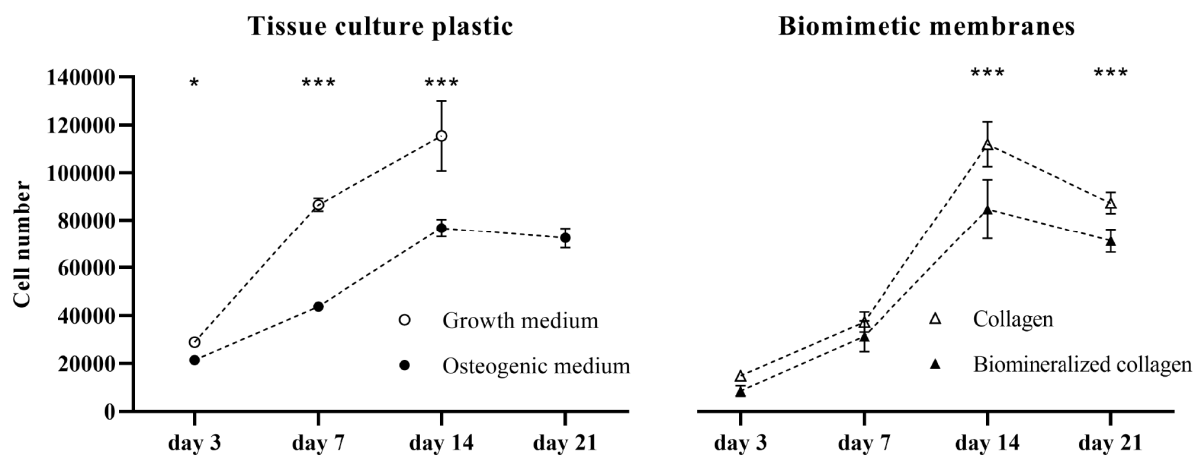

Figure 2. Proliferation of hMSCs on tissue culture plastic in GM and OM as well as on the biomimetic collagen and biomineralized collagen membranes in GM. Cell proliferation is expressed as cell number at 3, 7, 14 and 21 days of culture. The data point for 21 days in the GM condition is missing, due to cells becoming over-confluent and eventually detaching from the tissue culture plate. Data are represented as mean and standard deviation $(n=6)$ and significance is denoted as ${ }^{*} \mathrm{p}<0.05,{ }^{* * *} \mathrm{p}<0.001$.

\subsection{Expression of osteoclast-related genes by hMSCs on biomimetic membranes}

Potential modulation of osteoclast behavior by hMSCs cultured on collagen or biomineralized collagen was analyzed by assessing the expression of factors involved in osteoclastogenesis, including OPG, MCSF and RANKL ${ }^{[33,34]}$ at mRNA level (Figure 4). RANKL expression was below the detection limit of the qRT-PCR, indicating a very low expression level in all samples (Supplementary Figure 1). OPG expression in hMSCs on TCP was constant over time. An early upregulation at day 3 was observed on collagen, which was not statistically significant. An increase was also observed on mineralized collagen with a $3.5 x$ increase relative to TCP and $2 x$ increase relative to collagen, which were both statistically significant. At days 7 and 14, expression levels on collagen and mineralized collagen decreased to levels similar to those on TCP. MCSF expression at day 3 was significantly increased $4.5 \mathrm{x}$ on collagen and 5.5x on mineralized collagen relative to TCP. This trend, of a higher expression on both biomimetic membranes, was maintained at days 7 and 14 . Overall, however, MCSF expression decreased over time, from day 3 until day 14, on all substrates. 

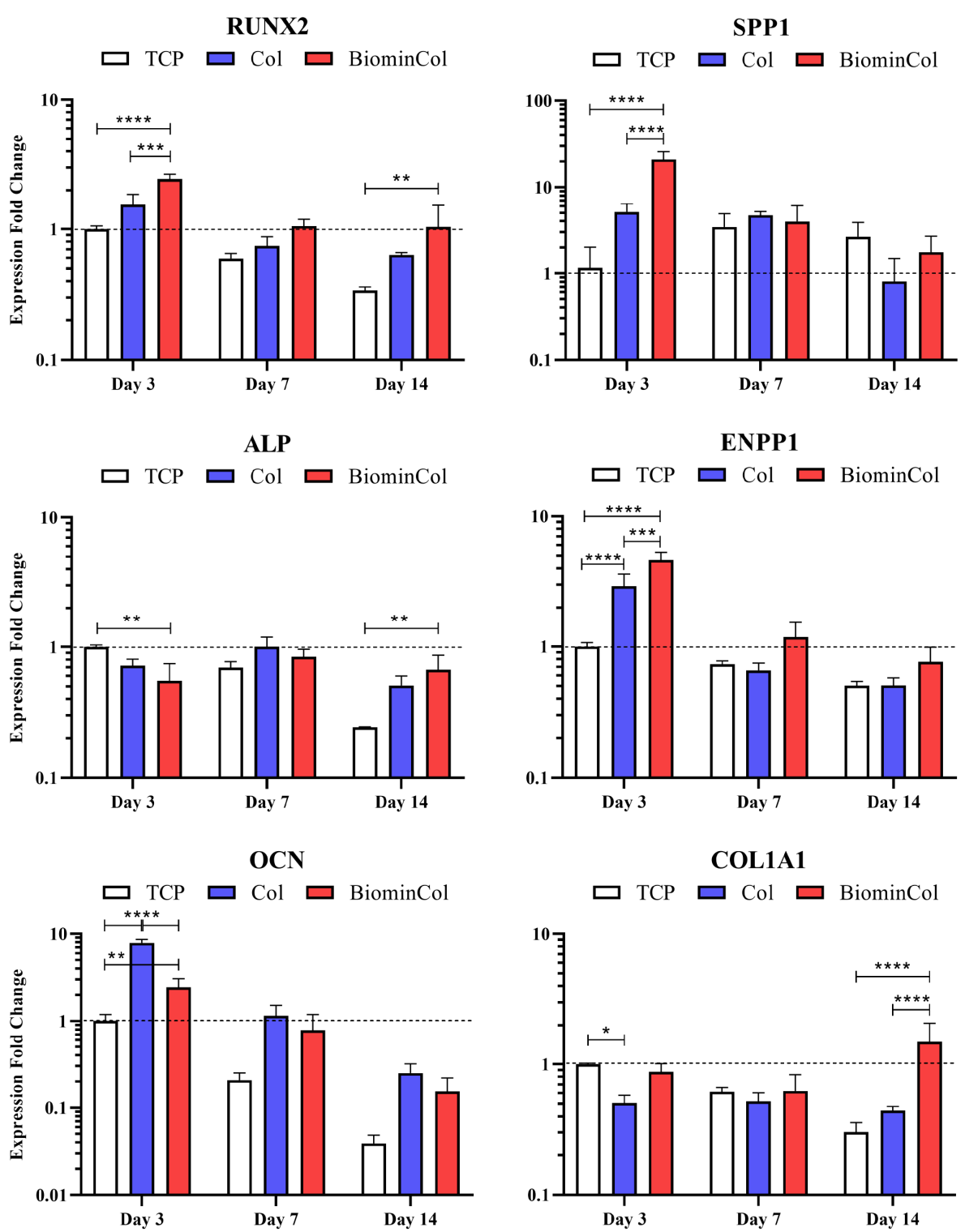

Figure 3. Gene expression profiles for RUNX2, SPP1, ALP, ENPP1, OCN, and COL1A1 of hMSCs cultured on tissue culture plastic (TCP) control, collagen (Col), and biomineralized collagen (BiominCol) for 3, 7, and 14 days. Data are represented as mean \pm SD $(n=3)$. Data is presented as mean and standard deviation $(n=3)$ and significance is denoted as ${ }^{*} p<0.05,{ }^{* *} p$ $<0.01,{ }^{* * *} \mathrm{p}<0.001,{ }^{* * * *} \mathrm{p}<0.0001$. 


\subsection{ALP activity}

In addition to assessing the mRNA expression levels of ALP, its activity was also analyzed as biochemical marker for osteoblast-like function in hMSCs undergoing osteogenic differentiation on different materials (Figure 5). ALP activity of hMSCs after 3 days was below the limit of detection for all conditions except the biomineralized collagen. On day 7, ALP activity was around two times higher on the biomineralized collagen than on the TCP control. Cells on mineralized collagen also showed a higher ALP activity than on collagen, although this difference was not statistically significant. There was a general increase from 7 to 14 days, and hMSCs cultured on both biomimetic membranes showed approximately twofold higher ALP activity than those cultured on TCP, while no significant effect of collagen mineralization was observed. Finally, on day 21, no measurement could be done on the TCP control, as cells grew over-confluent and samples had to be discarded. Collagen and biomineralized collagen showed comparable ALP activity on day 21, with a slight decrease compared to day 14 .

\subsection{Cell-induced mineralization}

Production of mineralized ECM by the hMSCs was evaluated by SEM and EDS analyses of membranes after 21 days of cell culture in MM. Mineral deposits produced by hMSCs were observed on collagen membranes (Figure 6A). The mineral deposits were found as a layer surrounding the collagen fibers of the membrane (inset Figure 6A), in close proximity to the cells on the membrane. These deposits were also found in the areas of the collagen membrane where no cells were observed. On mineralized collagen membranes, the only possible indication of cellinduced mineralization were spherical structures with the diameter of a few micrometers, which were observed in close association with cell filopodia (Figure 6B). Elemental analysis by EDS showed presence of calcium and phosphorus on the collagen membranes after cell culture in MM (Figure 7A), and elemental mapping showed that the signal was originating from the mineral deposits (Figure 7B-E). No mineral deposits were observed by SEM and no calcium or phosphorus presence was detected by EDS when hMSCs were cultured on collagen membranes in GM for 21 days (Supplementary Figure 2). Similarly, no evidence for mineral deposits was 
found when collagen membranes were incubated in MM for 14 days in the absence of cells (Supplementary Figure 3).
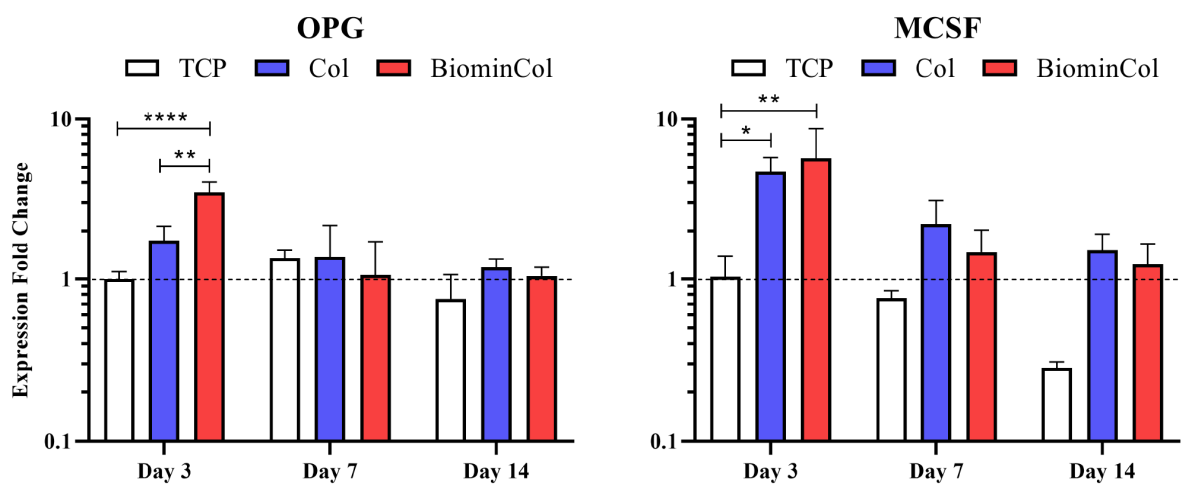

Figure 4. Gene expression profiles for OPG and MCSF of hMSCs cultured on tissue culture plastic (TCP) control, collagen (Col), and biomineralized collagen (BiominCol) for 3, 7, and 14 days. Data is presented as mean and standard deviation $(n=3)$ and significance is denoted as ${ }^{*} \mathrm{p}<0.05,{ }^{* *} \mathrm{p}<0.01,{ }^{* * * *} \mathrm{p}<0.0001$.

\section{Discussion}

The intrinsic self-healing capacity of bone falls short in repairing critical-sized bone defects. To avoid drawbacks commonly associated with using bone grafts, such as limited availability as well as donor site morbidity, ${ }^{[1,2]}$ synthetic bone graft substitutes are in demand as replacement for autologous bone. ${ }^{[3,4]}$ Intrafibrillary biomineralized collagen is a promising biomaterial for bone regeneration applications, as it mimics not only the organic-inorganic composition, but also the structure of the ECM of bone tissue at the sub-micron scale. In this study, we aimed to complement the existing knowledge on the relevance of biomineralized collagen in bone regeneration, by evaluating the behavior of clinically-relevant hMSCs on intrafibrillarly mineralized collagen membranes in comparison with collagen membranes without mineral. The differentiation of undifferentiated stem cells into osteogenic progenitors, i.e., osteoinduction, is a key process in regeneration of bone defects $^{[35]}$ and gaining further insight into this process is a valuable resource for designing novel and more functional bone graft substitutes. There are only a few in vitro studies exploring the effects of biomineralized collagen on osteogenic differentiation of human stem cells.[19-22] The results with hMSCs described here 
contribute to the growing evidence that this biomaterial is a promising candidate for developing synthetic bone graft substitutes.

Biomineralized collagen membranes, consisting of collagen type I containing intrafibrillar CaP mineral, were prepared following the established PILP route.[10] Dense type I collagen membranes had a fibrillar structure with typical D-banding characteristic of native collagen, a requirement for intrafibrillar mineralization to occur. ${ }^{[6]}$ Upon mineralization of the membranes, the fibrillar structure of the membrane was preserved. Typical crystal deposition from saturated $\mathrm{Ca}^{2+}$ and $\mathrm{PO}_{4}{ }^{3-}$ solutions ${ }^{[36]}$ was not observed on the surface of the material, while the elemental analysis showed that $\mathrm{CaP}$ was present in abundance. These observations indicate that $\mathrm{CaP}$ was present in the mineralized collagen membrane, but not on the surface of the fibers, and therefore must reside within the collagen fibers. Further evidence of this is the observed increase in fiber diameter after the biomineralization process, being roughly three times the size of the collagen membrane fibers. We therefore conclude that homogenous crystallization, i.e., crystallization in solution, did not occur, and mineral was instead deposited within the collagen fibrils, which is expected when a polyanion such as $\mathrm{pAsp}$ is added to the mineralization process, and is in accordance with previous studies in which PILP was used for collagen biomineralization. ${ }^{[37,38]}$

Mineralized collagen membranes were used to study proliferation and differentiation of hMSCs at the mRNA level, using collagen membranes without mineral as a control. Moreover, ALP activity and mineralization of the cells were analyzed.

Both biomimetic membranes with and without mineral supported the growth of hMSCs over a period of 21 days, with mineralized collagen showing a somewhat lower proliferation rate, in particular at the later time points of 14 and 21 days. Previous studies have also shown that collagen matrices with and without $\mathrm{CaP}$ mineral support cell proliferation. For example, in a study with MC3T3-E1 osteoblasts, a more pronounced proliferation on collagen without than on collagen containing mineral was observed, however, this analysis was only performed over a period of 7 days. ${ }^{[15]}$ Collagen with and without HA supported proliferation of rat MSCs over a period of 14 days without significant differences between the materials, ${ }^{[39]}$ whereas periodontal ligament stem cells were present in higher 
numbers on intrafibrillarly mineralized collagen than on the collagen control at 3, but not at 7 days of culture. ${ }^{[20]}$

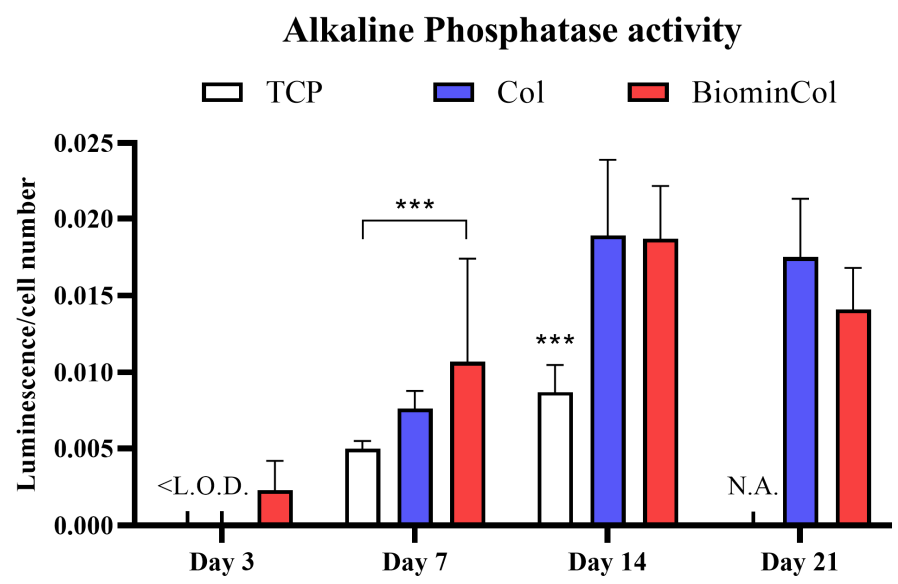

Figure 5. ALP activity of hMSC, normalized to cell number. Cells were cultured on tissue culture plastic (TCP) control, collagen ( $\mathrm{Col})$, and biomineralized collagen (BiominCol) for 3 , 7, 14, and 21 days. Data under the limit of detection are denominated as <L.O.D (growth medium and collagen at day 3), and missing data is labeled as N.A. (TCP control at day 21). Data is presented as mean and standard deviation $(n=6)$ and significance is denoted as ${ }^{* * *} \mathrm{p}$ $<0.001$.

Regarding osteogenic differentiation, in general, hMSCs showed an upregulation of osteogenic differentiation markers when cultured on mineralized collagen as compared to collagen without mineral.

RUNX2, an essential transcription factor required for determination of the osteoblastic lineage in hMSC at an early stage ${ }^{[27]}$ as well as SPP1, another early marker of osteogenesis, that has been shown to be expressed in immature osteoblasts, ${ }^{[28]}$ were enhanced at an early time point on biomineralized collagen membranes. This effect on RUNX2 was maintained, however, at lower mRNA levels and decreased for SPP1 over time. We observed a similar trend in time on collagen, however, the expression of RUNX2 was always lower than on biomineralized collagen indicating a lower degree of osteogenic differentiation. An early upregulation of RUNX2, followed by a slow decrease, is a pattern found over the course of osteogenic differentiation of hMSCs in vitro. ${ }^{[40]}$ RUNX2 is regulated by several upstream pathways, which are involved in osteogenic differentiation, 
including BMP and WNT signaling pathways. ${ }^{[41]}$ Its upregulation indicates an activation of these pathways and, hence, material-induced osteogenic differentiation. This also explains the upregulation of SPP1, which is a direct target of RUNX2 signaling. ${ }^{[27]}$ SPP1 is an important factor of matrix mineralization, which also plays a role in many other functions such as cell survival, migration, regulation of inflammation and angiogenesis. ${ }^{[42]}$

ALP and ENPP1 are known to positively and negatively regulate matrix mineralization in native bone, ${ }^{[31,32]}$ respectively. The mRNA level of ALP was lower, while the ENPP1 mRNA was higher on biomineralized collagen compared to the control at day 3. The opposite trend in ALP levels overtime was observed, with cells cultured on TCP showing a significant decrease, while biomineralized collagen maintained the level comparable to that at day 3. A substantial decrease of overall ENPP1 expression was observed at later time points, plausibly indicating the onset of matrix mineralization. ALP and ENPP1 expression of cells cultured on the collagen membranes and biomineralized collagen were similar, with ENPP1 expression being slightly lower in the absence of mineral. ENPP1 expression has previously been shown to be upregulated on $\mathrm{CaP}$ ceramics with intrinsic osteoinductive potential.[43] To our knowledge, this is however, the first time that ENPP1 expression has been investigated on collagen and biomineralized collagen. OCN, the most abundant non-collagenous protein in bone, ${ }^{[30]}$ was surprisingly expressed at an early stage with an upregulation on biomineralized collagen compared to the control. This trend was maintained over time and a similar effect on OCN expression was observed on collagen membranes without mineral. OCN has been shown to play an important role in osteoblast maturation, inter alia, promoting matrix mineralization by binding to HA. ${ }^{[44]} \mathrm{A}$ study by Tsao et al. showed that low $\mathrm{OCN}$ levels potentially lead to a delayed matrix mineralization. ${ }^{[30]}$ An early expression of OCN as observed here may indicate that biomimetic collagen membranes are able to accelerate osteogenic differentiation, however further research is needed to provide conclusive evidence for this.

COL1A1, the main component of the organic matrix, was higher at day 14 on biomineralized collagen relative to TCP (3.4x) and collagen (4.9x), suggesting matrix deposition by cells of the osteoblastic lineage. ${ }^{[29]}$ In contrast, collagen membranes without mineral were unable to stimulate COL1A1 expression, which was 
downregulated early on and kept at baseline until later time points. This effect of pure collagen scaffolds has previously been observed in different cell types. ${ }^{[20,39]}$

In a study by Sun et al., osteogenic differentiation of rat MSCs on collagen versus a collagen/HA composite, without intrafibrillar mineral, was tested, showing the results consistent with those presented here. ${ }^{[39]}$ Cells cultured on collagen/HA scaffolds showed, overall, the highest osteogenic differentiation capacity, supported by a rapid activation of RUNX-2 and the high mRNA levels of ALP and especially COL1A1. The authors attributed these effects to the differences in microstructure and stiffness of the materials. ${ }^{[39]}$ An upregulation of RUNX2 was also observed on $\mathrm{CaP}$ ceramic controls without collagen and may therefore also be influenced by the presence of $\mathrm{CaP}$ and/or free calcium and inorganic phosphate ions.

mRNA expression of ALP in hMSCs cultured on collagen and mineralized collagen has been sparsely investigated. However, a study investigating osteogenic differentiation of hMSCs on nanoparticulate (mineralized) collagen glycosaminoglycan scaffolds showed a low ALP expression at day 3 and a slight increase at day 7.45] Both scaffolds with and without mineral also showed an upregulation in matrix markers, such as OCN and COL1A1, as compared to the beginning of culture (day 0 ), which is in accordance with our results. In a study by Fu et al., osteogenic differentiation of human periodontal ligament stem cells was evaluated on collagen, intrafibrillarly mineralized collagen and extrafibrillarly mineralized collagen. ${ }^{[20]}$ Cells cultured on intrafibrillarly mineralized collagen showed an increased expression of osteogenic genes, such as SPP1, COL1A1 and BMP-2 as compared to collagen at days 7 and 14 .

In the context of bone regeneration, not only the osteoblast function, as the boneforming unit, but also the osteoclast function, the bone-resorbing unit, is of importance. In native bone, these two processes are in balance, maintaining a constant, homeostatically-controlled amount of bone ECM.[46] Therefore, in this study, we took first steps in analyzing how biomimetic membranes may influence osteoblast-osteoclast communication by assessing the mRNA levels of the biomarkers involved in this cellular crosstalk in hMSCs.

The RANKL/OPG ratio has an important role in osteoclast regulation. RANKL activates osteoclasts by binding to its membrane-bound receptor RANK, and OPG has an inhibitory effect by binding RANKL to prevent osteoclast activation. ${ }^{[33]}$ As reviewed by Kapasa et al., previous research indicated that changes in RANKL/OPG 
balance might reduce implant complications, which are caused by an increased osteoclastogenesis accompanied by chronic inflammation in which significantly elevated levels of RANKL are frequently observed. ${ }^{[47]}$ This results in poor osseointegration of the implant, prosthetic loosening and non-union of the defect. Here, low mRNA levels of RANKL in hMSCs were detected overtime. Osteoblasts can exhibit a pro-osteoclastic phenotype, expressing RANKL. This was previously observed in an immature subpopulation of osteoblasts and simulated in vitro by the stimulation with osteotropic factors vitamin D3, dexamethasone or their combination. ${ }^{[48]}$ Here, however, low mRNA levels of RANKL were detected overtime. Ren et al. combined OPG adenoviral expression in hMSCs with nanoparticulate mineralized collagen glycosaminoglycan scaffolds or nonmineralized scaffolds and observed a decreased RANKL/OPG ratio.[49] RANKL expression was detected not only in OPG transfected hMSCs but also in nontransfected hMSCs on mineralized and non-mineralized material. The major difference in Ren et al.' culture protocol compared to ours was the culture of hMSCs in osteogenic medium containing dexamethasone. We speculate that the lack of dexamethasone in our cultures, chosen to isolate the effect on the biomaterials from other stimulators of osteogenic differentiation, may be the reason for the low RANKL expression observed.

OPG mRNA expression, on the other hand, was upregulated in hMSCs cultured on biomineralized collagen indicating the potential of this biomaterials to modulate the osteoblast-osteoclast crosstalk.

Jiao et al. observed that mouse MSCs cultured on biphasic silica/apatite intrafibrillarly mineralized collagen scaffolds expressed higher mRNA levels of OPG (5-fold increase), and lower mRNA levels of RANKL relative to collagen scaffolds without mineral.[16] OPG expression on intrafibrillarly calcified collagen scaffolds without silica was only slightly increased and no difference in RANKL expression relative to collagen without mineral was observed. This indicated an inhibitory effect on RANKL-mediated osteoclastogenesis induced by biphasic silica/apatite intrafibrillarly mineralized collagen. This hypothesis was confirmed in a follow up experiment in which osteoclasts were exposed to conditioned medium from hMSCs cultured on these scaffolds, finally resulting in decreasing numbers of functional osteoclasts, i.e tartrate-resistant acid phosphatase (TRAP)-positive cells. 
In our study, we additionally observed an upregulation of MCSF mRNA expression, which has been suggested to activate osteoclastic bone resorption, ${ }^{[34]}$ in $\mathrm{hMSCs}$ cultured on both collagen and biomineralized collagen membranes, with the effect remaining elevated compared to control, yet overall, decreasing overtime.

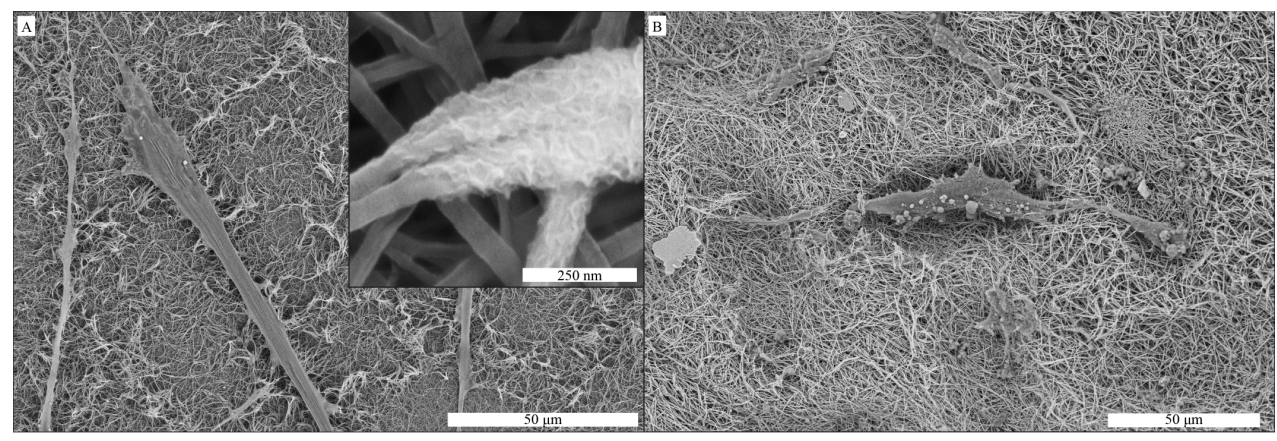

Figure 6. Cell-induced mineralization on collagen (A) and biomineralized collagen (B) membranes, shown by SEM imaging. Cells were cultured on membranes for 21 days in MM. Mineral deposits are visible in the collagen membrane (inset A). These deposits were not observed on biomineralized collagen (B).

Taken together, our data suggests that collagen and biomineralized collagen can potentially modulate osteoclastogenesis and the osteoclast-osteoblast crosstalk during bone regeneration process. However, to gather a deeper understanding of these modulatory effects, further investigation including direct osteoclast monoculture as well as hMSCs-osteoclast co-culture on these bioinspired biomaterials are required and suggested as next steps.

In addition to mRNA expression levels of osteogenic biomarkers, we also analyzed ALP activity, as an indicator of osteoblastic function in hMSCs cultured on collagen and biomineralized collagen membranes. ALP is an enzyme found in osteoblasts which increases matrix mineralization. ${ }^{32,50]}$

The analysis of the mRNA ALP levels showed that overtime, the ALP expression of hMSCs cultured on TCP control decreased, whereas on collagen and mineralized collagen, no decrease was observed, and the levels at 7 and 14 days were comparable between the two materials. In contrast, an increase in ALP activity was observed between day 3 and day 14, and remained at comparable level until day 21, for hMSCs cultured on both biomimetic membranes as well as the TCP control. This suggests that the peak in the ALP expression at the mRNA level was reached early during the 
cell culture, and that the effect at the enzyme level is observed at the later time points. Both biomimetic membranes showed a higher ALP activity than the TCP control, with the positive effect of biomineralized collagen being more pronounced at day 3 and day 7. This suggests an enhancing effect of the biomimetic matrices, and in particular the biomineralized one, on osteoblastic activity and, hence, evidence that hMSCs are differentiating into functional osteoblasts.

Previous studies have shown a higher ALP activity of cells cultured on intrafibrillarly mineralized collagen than on collagen without mineral[16,18,21] and even on collagen with extrafibrillar mineral.[17] These findings are in line with ours, although we did not observe significant differences between the two biomimetic membranes. This may be due to differences in the materials used in different studies. Although all studies tested intrafibrillarly mineralized collagen, slight differences in method used for preparing biomineralized collagen could have an impact on important properties of the materials. For example, the extent of exposure of the intrafibrillar mineral from its collagen confinement may be different, affecting both the chemical composition, mechanical properties, and surface topography, which in turn may affect the cell behavior. This is not only the case for the ALP activity but also for expression of other markers of osteogenic differentiation, cell proliferation and ECM production. Therefore, comparisons of different studies that tested biomineralized collagen need to be made with caution.

The formation of cell-induced mineral deposits on collagen and biomineralized collagen membranes was investigated by SEM-EDS. Mineral formation was microscopically observed on the collagen membranes, and supported by the elemental analysis demonstrating the presence of calcium and phosphorus. Critically, calcium and phosphorus were not detected on two controls, i.e., hMSCs cultured on the collagen membranes in GM and collagen membranes incubated in MM medium for 14 days in the absence of cells. These observations strongly suggest that the mineral deposits were indeed produced by the hMSCs cultured in MM, which contains dexamethasone and $\beta$-glycerophosphate. Moreover, the morphology of these deposits is interesting, as the mineral appears to surround the collagen fibers. This type of mineralization has been previously described for hMSC undergoing osteogenic differentiation in collagen matrices, where toluidine blue staining allowed identification of mineralized fibers, which were always found in contact with cells. [51] 
The high content of intrafibrillar $\mathrm{CaP}$ mineral in biomineralized membranes made detection of cell-deposited mineral difficult to analyze using SEM-EDS. On SEM images of biomineralized collagen membranes, we did not observe the same type of mineral deposits as seen on collagen membranes. Instead, round particles were observed surrounding the cells, the nature of which is unclear.

While it is reasonable to expect that hMSC cultured on biomineralized collagen in MM would produce mineralized matrix, ${ }^{[52]}$ we did not find convincing evidence for this. One reason for the lack of cell-induced mineral deposits, similar to the ones observed on collagen membranes, may be that the pre-existing intrafibrillar $\mathrm{CaP}$ could significantly alter the way in which new mineral is formed, by, for example, prioritizing growth of pre-existing $\mathrm{CaP}$ crystals over the formation of new mineral clusters. As existing CaP crystals are inside the collagen fibers, crystal growth would not significantly alter the fibrous morphology of the membrane, consequently making it more difficult to identify "new" mineral using SEM. However, if sufficient new mineral forms, the increase in fiber diameter should be detectable.

While other studies have used Alizarin Red semi-quantification to identify cellinduced mineral deposits, ${ }^{[20]}$ due to the high content of CaP in our biomineralized collagen membranes, this approach was not successful here (data not shown). Another alternative method for detecting newly formed mineral would be to tag calcium ions in solution with a fluorescent label, which would then be incorporated in newly formed mineral, making detection of deposits possible by fluorescence microscopy.[53]

Cell-induced matrix mineralization is an important aspect of de novo bone formation, and the capacity for hMSCs to mineralize the collagen membrane under stimulation of MM was demonstrated. It is reasonable to expect that hMSCs would show the same behavior when cultured on biomineralized collagen membranes, though we were unable to provide conclusive evidence here. 

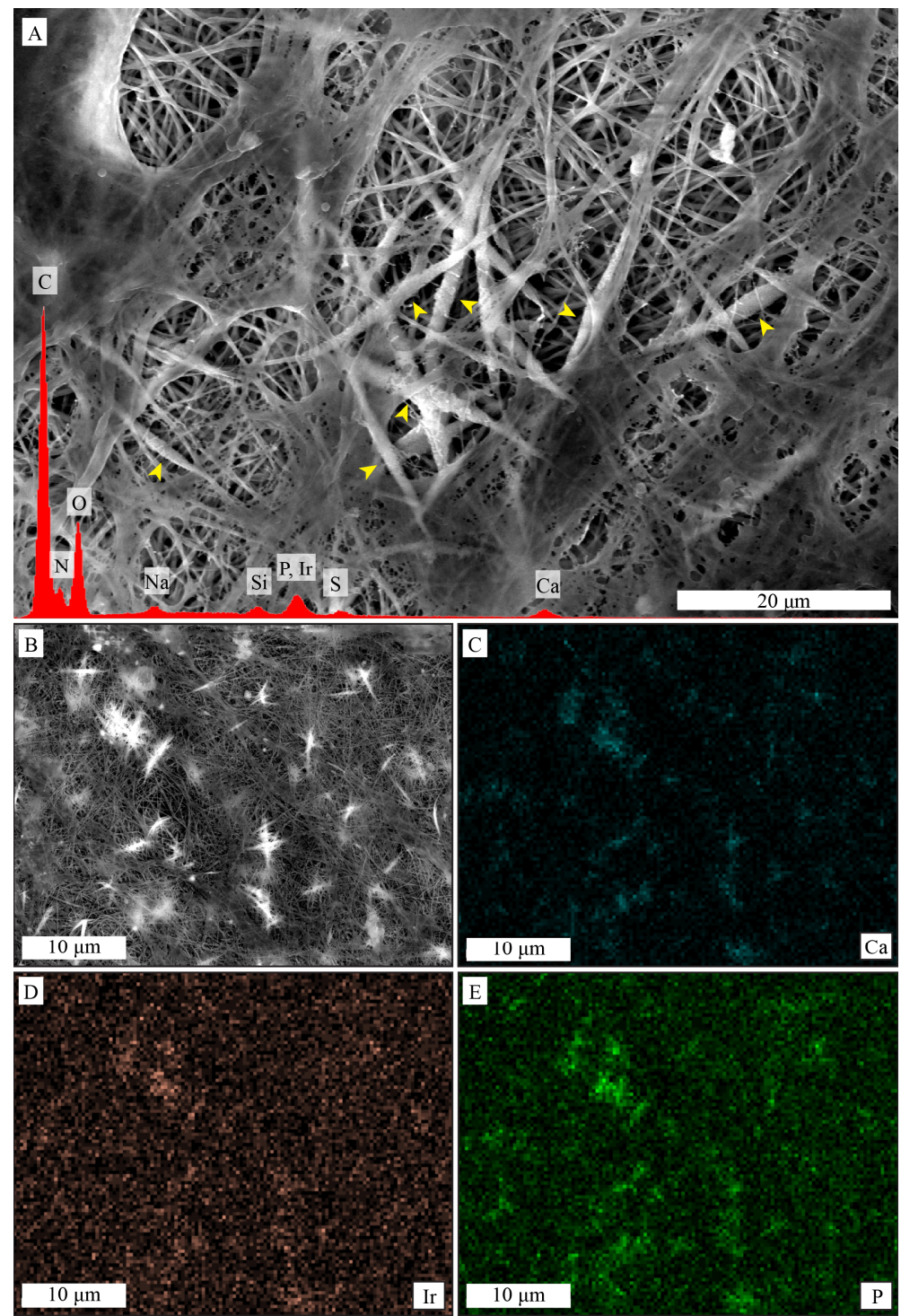

Figure 7. Cell-induced mineralization on collagen membranes, shown by SEM imaging and EDS analysis. Mineral deposits are visible as thicker and whiter fibers in the center of the image (A), yellow arrowheads. EDS spectra of (A), in red, shows presence of calcium and phosphorus. ESD mapping (B-E) shows that signal from calcium (C) and phosphorus (E) comes predominantly from the thicker and whiter mineral deposits that surround the collagen fibers, as seen in the previous figures. In contrast, the signal from iridium (D) is more homogeneous. 
Taken together, the results of this study that used bioinspired intrafibrillarly mineralized collagen and clinically relevant hMSCs, have shown that this material supports cell growth and osteogenic differentiation, and also affects markers related to osteoblast-osteoclast crosstalk. Moreover, the ability of the material to support cell-induced mineralization is suggested. While these findings add to the existing knowledge, a few limitations of this study should be discussed. First, the experiments were performed using a single hMSC donor and therefore, donor variability is not taken into account. Second, the induction of osteogenic differentiation was predominantly analyzed at the mRNA level. A next step to support the claim on enhancement of osteogenic differentiation on biomineralized collagen, is to assess the differentiation at the protein level and to further investigate the ECM deposition and mineralization. From a bone regeneration perspective, not only the formation of bone but also its resorption are relevant processes to be considered when developing a bone graft substitute. Our data on osteoblastosteoclast interaction indicated a possible modulatory effect of both collagen and mineralized collagen on osteoclastic functions and on osteoblast-osteoclast communication, which would be interesting to further explore. Lastly, production of the (mineralized) ECM by hMSCs on biomineralized collagen should be studied further, to clarify the nature of the mineral deposited and how this process is influenced by the properties of the material.

\section{Conclusions}

The results in this study demonstrate that biomineralized collagen membranes support the growth and osteogenic differentiation of hMSCs. The upregulation of the expression of osteogenesis-related genes such as RUNX2, SPP1, ENPP1, OCN, and COL1A1, as well as the enhancement of the ALP activity were observed. Furthermore, biomineralized collagen was shown to affect the expression of osteoclast-related genes OPG and M-CSF. hMSCs were able to deposit mineralized ECM on the collagen membranes and were suggested to have the ability to do the same on biomineralized collagen. We conclude that biomineralized collagen is a promising biomaterial for bone regeneration and merits further study. 


\section{Data availability statement}

The raw data generated for this study are publicly available at https://hdl.handle.net/10411/BC3SR3.

\section{Acknowledgments}

The authors would like to thank Myraise van Stijn and Enabling Technologies/DSM for the assistance provided with SEM-EDS analyses.

\section{Author Contributions}

DP designed the experiments, prepared biomaterials and performed cell culture, DNA and ALP quantification, SEM-EDS experiments and analyses. MJE performed qPCR experiments and analyses. DP and MJE wrote the manuscript. ZTB provided helpful insight during manuscript revision. PH supervised the project and reviewed the manuscript.

\section{Funding}

This research has been made possible with the support of the Dutch Province of Limburg (LINK project) and the Interreg Vlaanderen-Nederland 'Biomat on Microfluidic Chip' collaboration. PH gratefully acknowledges the Gravitation Program 'Materials-Driven Regeneration', funded by the Netherlands Organisation for Scientific Research (NWO).

\section{Conflict of interest}

The authors confirm that there are no known conflicts of interest associated with this publication and there has been no significant financial support for this work that could have influenced its outcome.

\section{Ethics statement}

The studies involving human participants were reviewed and approved by the medical ethics committee of Medisch Spectrum Twente (K06-002). The patient provided written informed consent to participate in this study. 


\section{Supplementary Material}

\section{RANKL}

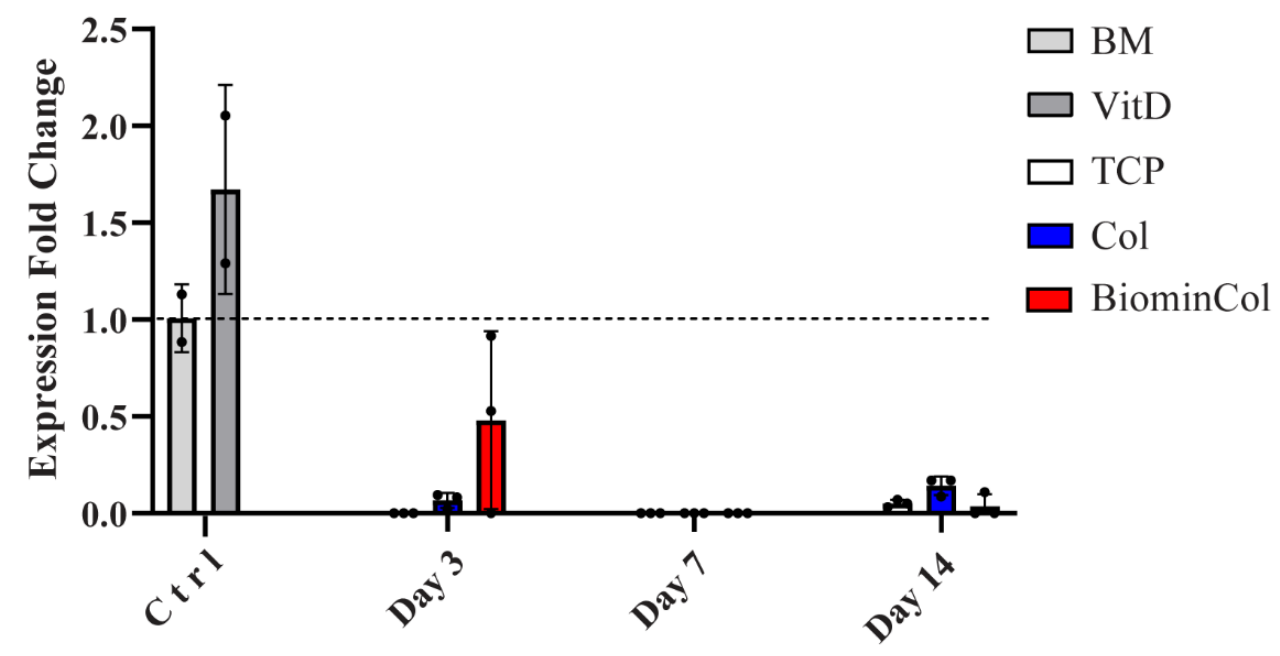

Supplementary Figure 1. Gene expression profile for RANKL. Osteoblasts in basic medium (BM) and osteoblasts stimulated with $20 \mathrm{nM}$ vitamin D3 (VitD3) were used as controls (Ctrl) to validate the RANKL primers. hMSCs cultured on tissue culture plastic (TCP) control, collagen (Col), and biomineralized collagen (BiominCol) for days 3, 7 and 14 days. Data are represented as mean and standard deviation ( $n=2$ for BM and VitD3; $n=3$ for hMSCs). 


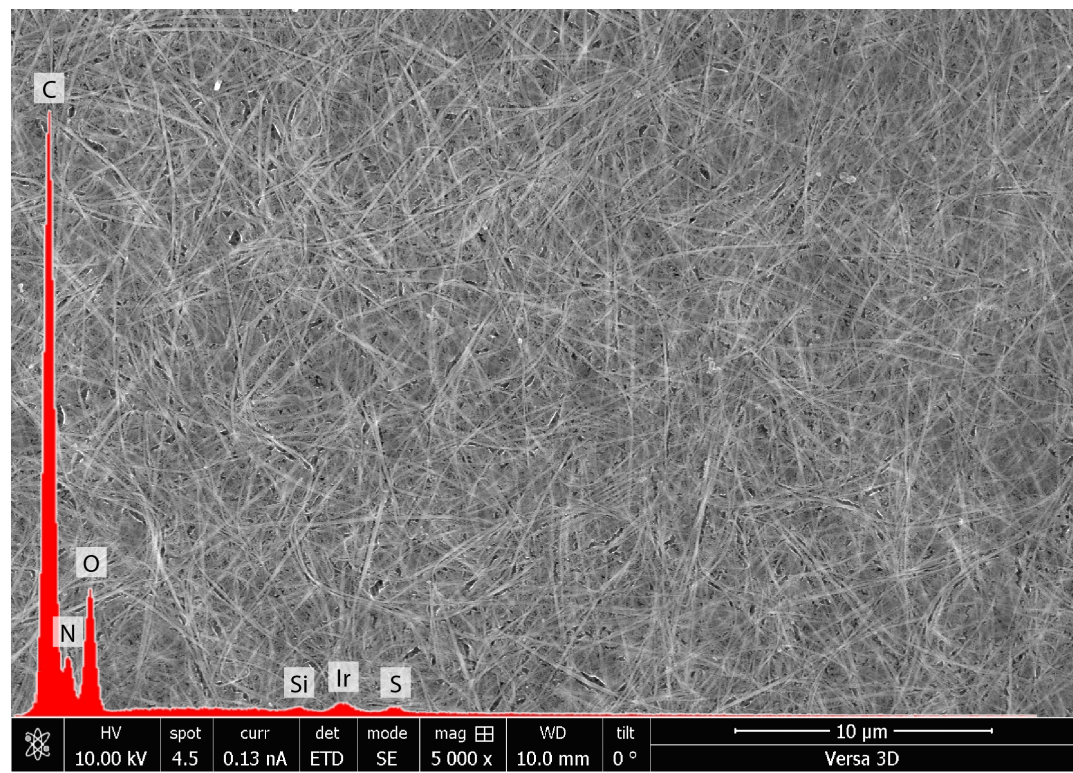

Supplementary Figure 2. SEM image of a collagen membrane with hMSCs cultured in GM for 21 days and EDS spectrum of the corresponding area. No calcium or phosphorus were detected.

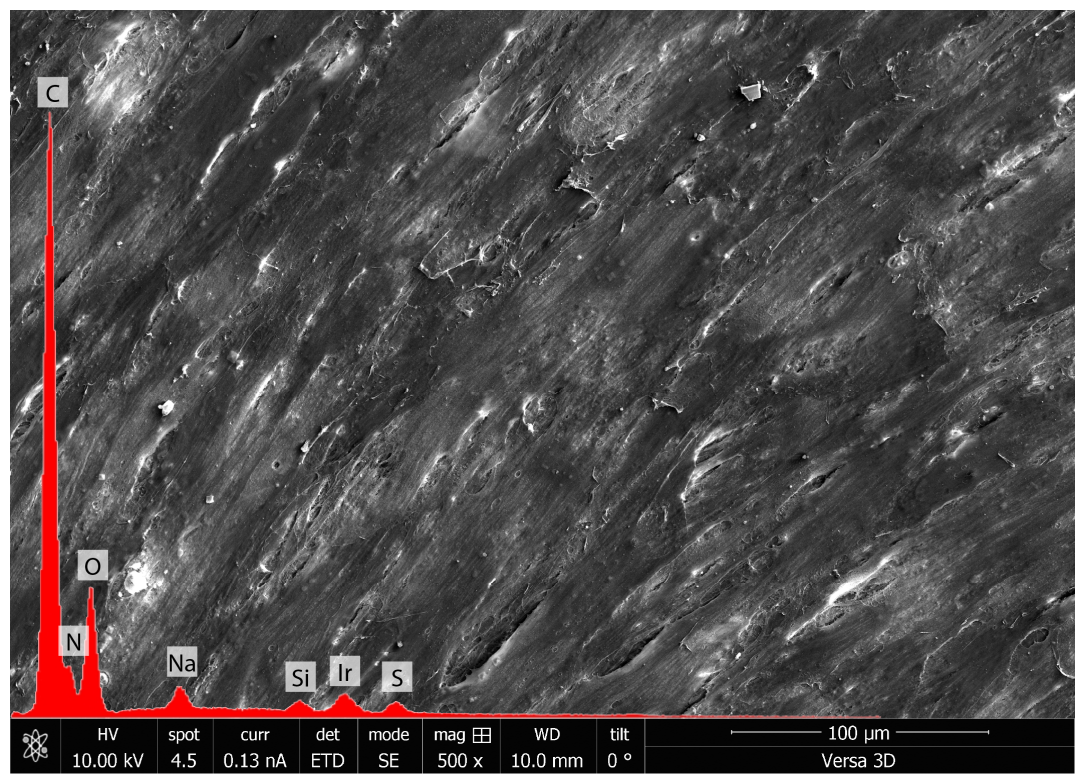

Supplementary Figure 3. SEM image of a collagen membrane incubated in MM for 14 days in the absence of cells (including media refreshment every 2-3 days) and EDS spectrum of the same area. No calcium or phosphorus were detected. 


\section{References}

[1] L. Babbi, A. Gasbarrini, S. Boriani, Eur. Rev. Med. Pharmacol. Sci. 2016, 20, 4670.

[2] G. M. Calori, M. Colombo, E. L. Mazza, S. Mazzola, E. Malagoli, G. V Mineo, Injury 2014, 45, S116.

[3] P. Baldwin, D. J. Li, D. A. Auston, H. S. Mir, R. S. Yoon, K. J. Koval, J. Orthop. Trauma 2019, 33, 203.

[4] T. Kurien, R. G. Pearson, B. E. Scammell, Bone Jt. J. 2013, 95 B, 583.

[5] O. A. Tertuliano, J. R. Greer, Nat. Mater. 2016, 15, 1195.

[6] M. J. Olszta, X. Cheng, S. S. Jee, R. Kumar, Y. Y. Kim, M. J. Kaufman, E. P. Douglas, L. B. Gower, Mater. Sci. Eng. R Reports 2007, 58, 77.

[7] N. Reznikov, M. Bilton, L. Lari, M. M. Stevens, R. Kröger, Science (80-. ). 2018, 360, eaao2189.

[8] Y. Li, C. Aparicio, PLoS One 2013, 8, 1.

[9] D. de Melo Pereira, P. Habibovic, Adv. Healthc. Mater. 2018, 1800700, 1.

[10] Y. Li, T. T. Thula, S. Jee, S. L. Perkins, C. Aparicio, E. P. Douglas, L. B. Gower, Biomacromolecules 2011, 13, 49.

[11] W. J. E. M. Habraken, J. Tao, L. J. Brylka, H. Friedrich, L. Bertinetti, A. S. Schenk, A. Verch, V. Dmitrovic, P. H. H. Bomans, P. M. Frederik, et al., Nat. Commun. 2013, 4, 1507.

[12] F. Nudelman, K. Pieterse, A. George, P. H. H. Bomans, H. Friedrich, L. J. Brylka, P. A. J. Hilbers, G. De With, N. A. J. M. Sommerdijk, Nat. Mater. 2010, 9, 1004.

[13] F. Nudelman, P. H. H. Bomans, A. George, G. de With, N. A. J. M. Sommerdijk, Faraday Discuss. 2012, 159, 357.

[14] Y. Liu, D. Luo, S. Liu, Y. Fu, X. Kou, X. Wang, Y. Sha, Y. Gan, Y. Zhou, J. Biomed. Nanotechnol. 2014, 10, 1049.

[15] Y. Wang, N. Van Manh, H. Wang, X. Zhong, X. Zhang, C. Li, Int. J. Nanomedicine 2016, 11, 2053.

[16] K. Jiao, L. Niu, Q. Li, F. Chen, W. Zhao, J. Li, J. H. Chen, C. W. Cutler, D. H. Pashley, F. R. Tay, Acta Biomater. 2015, 19, 23.

[17] Y. Wang, Y. Hua, Q. Zhang, J. Yang, H. Li, Y. Li, M. Cao, Q. Cai, X. Yang, X. Zhang, et al., J. Tissue Eng. Regen. Med. 2018, 12, 1545.

[18] Z. Zhang, Z. Li, C. Zhang, J. Liu, Y. Bai, S. Li, C. Zhang, Int. J. Nanomedicine 2018, 13, 7503.

[19] G. Thrivikraman, A. Athirasala, R. Gordon, L. Zhang, R. Bergan, D. R. Keene, J. M. Jones, H. Xie, Z. Chen, J. Tao, et al., Nat. Commun. 2019, 10, DOI 10.1038/s41467-01911455-8.

[20] Y. Fu, S. Liu, S. Cui, X. Kou, X. Wang, X. Liu, Y. Sun, G. Wang, Y. Liu, Y. Zhou, ACS Appl. Mater. Interfaces 2016, 8, 15958.

[21] B. Ye, X. Luo, Z. Li, C. Zhuang, L. Li, L. Lu, S. Ding, J. Tian, C. Zhou, Mater. Sci. Eng. C 2016, 68, 43.

[22] S. Xu, Z. Qiu, J. Wu, X. Kong, X. Weng, F. Cui, X. Wang, Tissue Eng. Part A 2016, 22, 170. 
[23] K. Lian, H. Lu, X. Guo, F. Cui, Z. Qiu, S. Xu, K. Lian, H. Lu, X. Guo, F. Cui, et al., Biomatter 2013, 3:e27250, DOI 10.4161/biom.27250.

[24] S. S. Liao, F. Z. Cui, W. Zhang, Q. L. Feng, J. Biomed. Mater. Res. - Part B Appl. Biomater. 2004, 69, 158.

[25] H. Fernandes, A. Mentink, R. Bank, R. Stoop, C. van Blitterswijk, J. de Boer, Tissue Eng. Part A 2010, 16, 1693.

[26] S. K. Booth, A. J. C. van der Muijsenberg, C. A. van Blitterswijk, J. de Boer, J. de Bruijn, Tissue Eng. 2007, 13, 3.

[27] P. Ducy, R. Zhang, V. Geoffroy, A. L. Ridall, G. Karsenty, Cell 1997, 89, 747.

[28] T. Komori, J. Cell. Biochem. 2006, 99, 1233.

[29] L. D. Quarles, D. A. Yohay, L. W. Lever, R. Caton, R. J. Wenstrup, J. Bone Miner. Res. 1992, 7, 683.

[30] Y. T. Tsao, Y. J. Huang, H. H. Wu, Y. A. Liu, Y. S. Liu, O. K. Lee, Int. J. Mol. Sci. 2017, 18, DOI 10.3390/ijms18010159.

[31] K. Johnson, J. Goding, D. V. a N. Etten, A. Sali, S. Hu, L. Milla, D. Farley, H. Krug, L. Hessle, R. Terkeltaub, J. Bone Miner. Res. 2003, 18, 994.

[32] L. Hessle, K. A. Johnson, H. C. Anderson, S. Narisawa, A. Sali, J. W. Goding, R. Terkeltaub, J. L. Millán, Proc. Natl. Acad. Sci. U. S. A. 2002, 99, 9445.

[33] J. C. Lee, L. Spiguel, D. Shenaq, M. Zhong, C. Wietholt, T.-C. He, R. R. Reid, Plast. Reconstr. Surg. 2010, 126, 100.

[34] R. L. Lees, J. N. M. Heersche, J. Bone Miner. Res. 1999, 14, 937.

[35] M. Bohner, R. J. Miron, Mater. Today 2019, 22, 132.

[36] D. O. Costa, B. A. Allo, R. Klassen, J. L. Hutter, S. J. Dixon, A. S. Rizkalla, Langmuir 2012, 28, 3871.

[37] S. Jee, T. T. Thula, L. B. Gower, Acta Biomater. 2010, 6, 3676.

[38] T. T. Thula, F. Svedlund, D. E. Rodriguez, J. Podschun, L. Pendi, L. B. Gower, Polym. Basel 2011, 3, 10.

[39] X. Sun, W. Su, X. Ma, H. Zhang, Z. Sun, X. Li, Regen. Biomater. 2018, 5, 93.

[40] A. Shekaran, E. Sim, K. Y. Tan, J. K. Y. Chan, M. Choolani, S. Reuveny, S. Oh, BMC Biotechnol. 2015, 15, 1.

[41] A. Rutkovskiy, K.-O. Stensløkken, I. J. Vaage, Med. Sci. Monit. Basic Res. 2016, 22, 95.

[42] C. M. Giachelli, S. Steitz, Matrix Biol. 2000, 19, 615.

[43] Z. Othman, H. Fernandes, A. J. Groot, T. M. Luider, A. Alcinesio, D. de M. Pereira, A. P. M. Guttenplan, H. Yuan, P. Habibovic, Biomaterials 2019, 210, 12.

[44] P. V. Hauschka, F. H. Wians, Anat. Rec. 1989, 224, 180.

[45] X. Ren, V. Tu, D. Bischoff, D. W. Weisgerber, M. S. Lewis, D. T. Yamaguchi, T. A. Miller, B. A. C. Harley, J. C. Lee, Biomaterials 2016, 89, 67.

[46] G. A. Rodan, Commentary 1998, 95, 13361.

[47] E. Kapasa, P. Giannoudis, X. Jia, P. Hatton, X. Yang, J. Funct. Biomater. 2017, 8, 42.

[48] G. J. Atkins, P. Kostakis, B. Pan, A. Farrugia, S. Gronthos, A. Evdokiou, K. Harrison, D. M. Findlay, A. C. W. Zannettino, J. Bone Miner. Res. 2003, 18, 1088.

[49] X. Ren, Q. Zhou, D. Foulad, A. S. Tiffany, M. J. Dewey, D. Bischoff, T. A. Miller, R. R. Reid, T. C. He, D. T. Yamaguchi, et al., Sci. Adv. 2019, 5, 1.

[50] R. S. Siffert, J. Exp. Med. 1951, 93, 415. 
[51] S. Neuss, R. Stainforth, J. Salber, P. Schenck, M. Bovi, R. Knüchel, A. Perez-Bouza, Cell Transplant. 2008, 17, 977.

[52] F. Langenbach, J. Handschel, Stem Cell Res. Ther. 2013, 4, DOI 10.1186/scrt328.

[53] S. M. van Gaalen, M. C. Kruyt, R. E. Geuze, J. D. de Bruijn, J. Alblas, W. J. A. Dhert, Tissue Eng. Part B. Rev. 2010, 16, 209. 


\section{Chapter 6}

\section{Behind the scenes - insights into technical details behind experimental chapters}

Daniel de Melo Pereira, Pamela Habibović 


\begin{abstract}
This chapter comprises a collection of experiments and the corresponding results that, while not part of any particular study or published articles, influenced choices made in all the experimental chapters presented so far. They are divided into optimization of parameters for intrafibrillar mineralization, production of the biomineralized collagen membranes, and monocyte/macrophage cell culture and differentiation to osteoclasts. The data presented here provide justification for (technical) choices made in the various chapters. Moreover, these results may be useful to the researcher wishing to replicate and build upon the experiments described in this thesis.
\end{abstract}

\title{
1. Optimization of parameters for intrafibrillar mineralization of collagen
}

As reviewed in Chapter 2, intrafibrillar mineralization of collagen can be achieved using a variety of polymers as substitutes for non-collagenous proteins found in bone (see their respective roles in Chapter 2, Table 1). These polymers can differ in chemical nature (e.g., be acidic, contain phosphate or serine moieties), molecular weight, and concentration used. Therefore, to enable efficient intrafibrillar mineralization, it was necessary to make a selection from a few candidates and determine optimal concentration for mineralizing type I bovine collagen from tendon, selected for our studies. Specifically, the aim was to prevent homogeneous precipitation of $\mathrm{CaP}$ in solution, while allowing a high degree of mineralization inside the collagen fibrils.

A first experiment was designed to test polyaspartic acid (sodium salt) of two different molecular weights (2-11 kDa and $27 \mathrm{kDa})$, polyaspartic acid (23 kDa), and polyacrylic acid (1800 Da). Polyaspartic acid was chosen because it is commonly used, and shown to produce large quantitates of intrafibrillar mineral in collagen from different sources.[1-17] Polyacrylic acid was used because data from the literature suggest that it prevents precipitation in solution, but it does not produce intrafibrillar mineral when used by itself. ${ }^{[18-20]}$ Each of the four polymers (Figure 1), was diluted in a range of concentrations $(0,10,50,100,200,300 \mu \mathrm{g} / \mathrm{mL})$ in a CaP solution (15 mL volume) typically used in the polymer-induced liquid precursor (PILP) method (150 mM NaCl, $50 \mathrm{mM}$ Tris, $4.5 \mathrm{mM} \mathrm{CaCl}$, $2.1 \mathrm{~K}_{2} \mathrm{HPO}_{4}$ ). At designated time-points, aliquots were taken from all conditions, transferred into a 96-well plate and their absorbance was determined in a plate reader. Turbidity 
measurements were compared with a control condition (no polymer) where precipitation occurred after a few minutes. For all polymers, an inhibition of precipitation was observed with an increase in concentration. At higher concentrations, precipitation was completely prevented until 7 days for all polymers (Figure 1). All conditions where absorbance remained close to 0 after 7 days effectively prevented precipitation in solution and were considered for a follow-up experiment involving collagen membranes.

pAsp 2-11 kDa

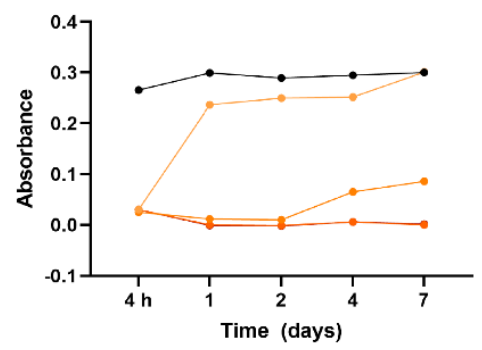

PAA 1800 Da

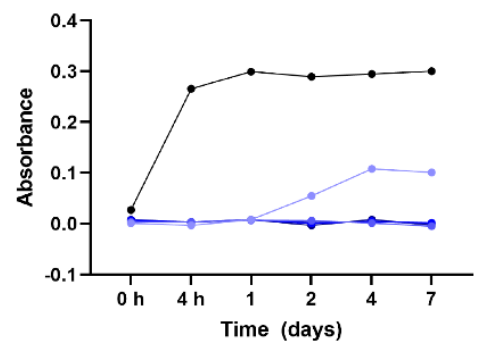

pAsp(H) 23 kDa

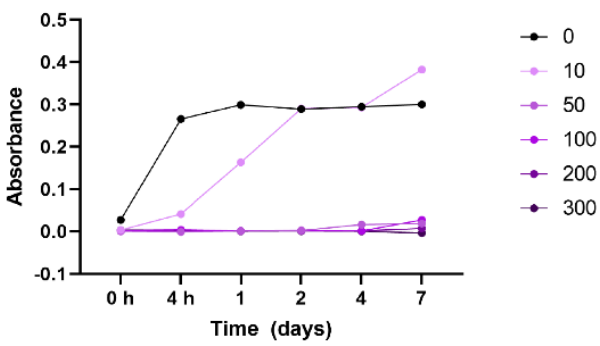

pAsp (Na) $27 \mathrm{kDa}$

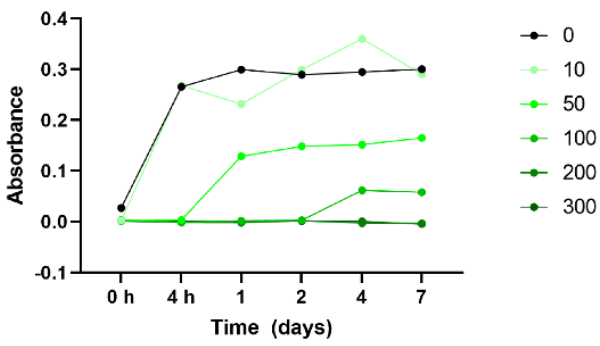

Figure 1. Turbidity of PILP mineralization solutions estimated by absorbance measurements at different time points over a period of 7 days at $37{ }^{\circ} \mathrm{C}$. Mineralization solutions consist of a Tris-saline buffer $(140 \mathrm{mM} \mathrm{NaCl}, 50 \mathrm{mM}$ Tris, $\mathrm{pH}=7.4)$, supplemented with $4.5 \mathrm{mM} \mathrm{CaCl}_{2}$ and $2.1 \mathrm{mM} \mathrm{Na}_{2} \mathrm{HPO}_{4}$ as well as with different polymer additives, in a range of concentrations. The control condition (in black) consists of the same mineralization solution without any added polymer, and in this condition, precipitation occurred in the solution after 4 hours of incubation. Measurements were taken from the same solution at different time points.

In this second experiment, collagen membranes were incubated for 7 days at $37{ }^{\circ} \mathrm{C}$ in the PILP solution with the various polymer formulations. Total mineral content was evaluated at the end of experiment by TGA, and pAsp (Na) $27 \mathrm{kDa}(50,100,200$ $\mu \mathrm{g} / \mathrm{mL})$ and PAA $1800 \mathrm{Da}(10 \mu \mathrm{g} / \mathrm{mL})$ showed highest mineral content, around 75 
wt.\% (Figure 1). The formulation chosen for all experiments was $100 \mu \mathrm{g} / \mathrm{mL}$ pAsp (Na). We discarded PAA $1800 \mathrm{Da}(10 \mu \mathrm{g} / \mathrm{mL})$ and pAsp(Na) $27 \mathrm{kDa}(50 \mu \mathrm{g} / \mathrm{mL})$ as possible alternatives due to the slight increase in turbidity observed in presence of either polymer before the end of the mineralizations period of 7 days (Figure 1). It is interesting to note that concentration differences for PAA $1800 \mathrm{Da}$ (10 vs 50 and 100 $\mu \mathrm{g} / \mathrm{mL}$ ) showed very different intrafibrillar mineralization results. In contrast to 10 $\mu \mathrm{g} / \mathrm{mL}$, at the higher concentrations, no mineral formation occurred.

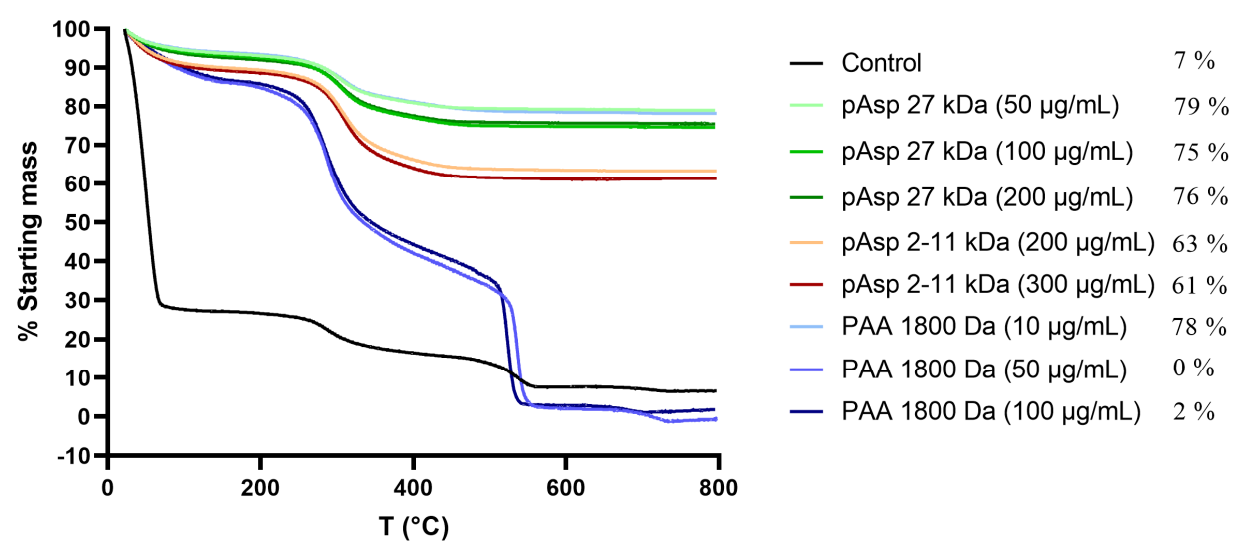

Figure 2. TGA analysis for determination of total mineral content of biomineralized collagen membranes prepared by the PILP method using different polymer additives in a range of concentrations. Samples were heated up to $800^{\circ} \mathrm{C}$, at a rate of $5^{\circ} \mathrm{C} / \mathrm{min}$ in air. The leftover mass percentage at $800{ }^{\circ} \mathrm{C}$ is registered next to each condition in the legend, and represents the inorganic mass fraction of the composite.

\section{Comparison of roughness between biomineralized collagen membranes and cortical bone}

Surface structure and topographical features can have an influence on osteoclast differentiation and function, as discussed in Chapter 3 and Chapter 4. Here we provide information on roughness parameters of the biomineralized collagen membranes used in the experimental chapters, compared side-by-side with bovine cortical bone surfaces, where monocytes/macrophages readily differentiate into osteoclasts with normal function.

Data was obtained by 3D laser profilometry of dry samples (Figure 3). In general, we observed a slightly more rough and uneven surface of biomineralized collagen, characterized by higher peaks and valleys, than that found on bone slices. Roughness (Sq) was below $1 \mu \mathrm{m}$ on the bone slices and close to $2 \mu \mathrm{m}$ on 
biomineralized collagen. The peak height (Sp) was twice as high on biomineralized collagen, and valley depth (Sv), although closer to that of bone slices, was also larger on biomineralized collagen. We considered the differences in surface-structural properties to be small enough not to warrant any modifications in the processing of collagen membranes, but kept in mind that their potential effect on osteoclast behavior cannot be fully excluded. Small variations in roughness (few micrometers) at the same scale were shown to have an effect on sealing zone stability and translocation on titanium surfaces.[21]
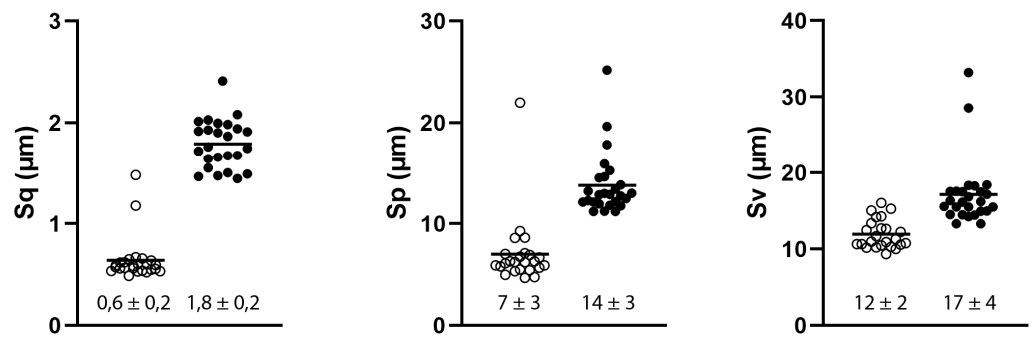

- Cortical bone slice

- BiominCol
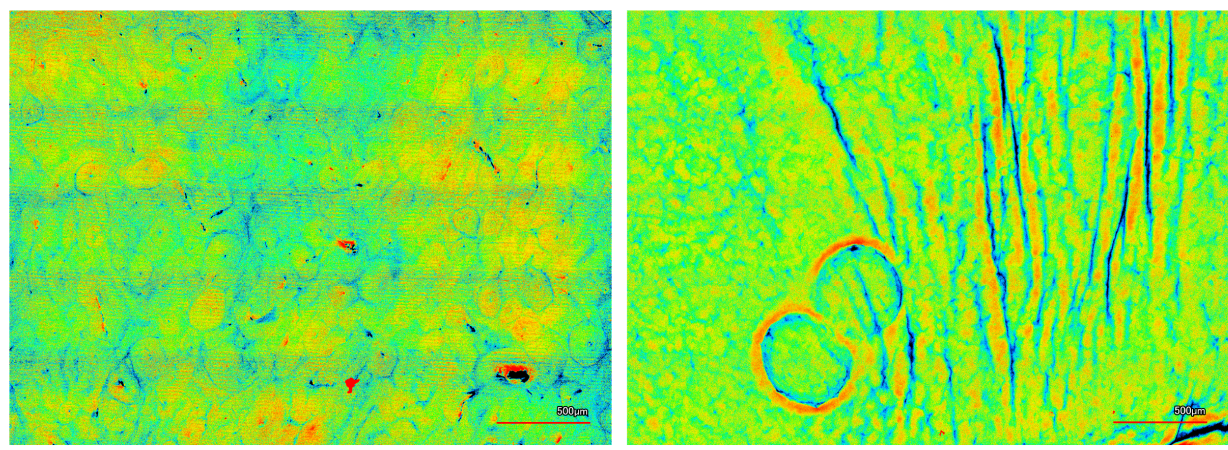

Figure 3. Roughness parameters of cortical bone slice and biomineralized collagen measured by 3D laser profilometry. Roughness parameters Sq (root mean squared height), Sp (maximum peak height), and Sv (maximum valley height) were averaged from independent fields-of-view for each sample $(n=25)$, and are presented as mean \pm standard deviation $(\mu \mathrm{m})$ in each graph. Biomineralized collagen has on average higher roughness $(3 x)$, higher peaks $(2 \mathrm{x})$, and lower valleys $(1.4 \mathrm{x})$ than cortical bone slice. 
3. Inclusion of metallic ions in the intrafibrillar mineral phase of biomineralized collagen

The presence of trace amounts of certain ions in biomaterials for bone regeneration can be desirable, not only from a biomimetic perspective, but also from a functional standpoint, as bioactive agents able to trigger certain cell behavior. ${ }^{[22]}$ This was discussed in Chapter 2, and exemplified in Chapter 4, with the inclusion of cobalt ions in the $\mathrm{CaP}$ phase of biomineralized membranes.

We attempted inclusion of cations of cobalt $\left(\mathrm{Co}^{2+}\right)$ (Figure 4), strontium $\left(\mathrm{Sr}^{2+}\right)$ (Figure 5) and magnesium $\left(\mathrm{Mg}^{2+}\right)$ (Figure 6) directly in intrafibrillar mineral by adding their salts to the calcium precursor solution in different concentrations.
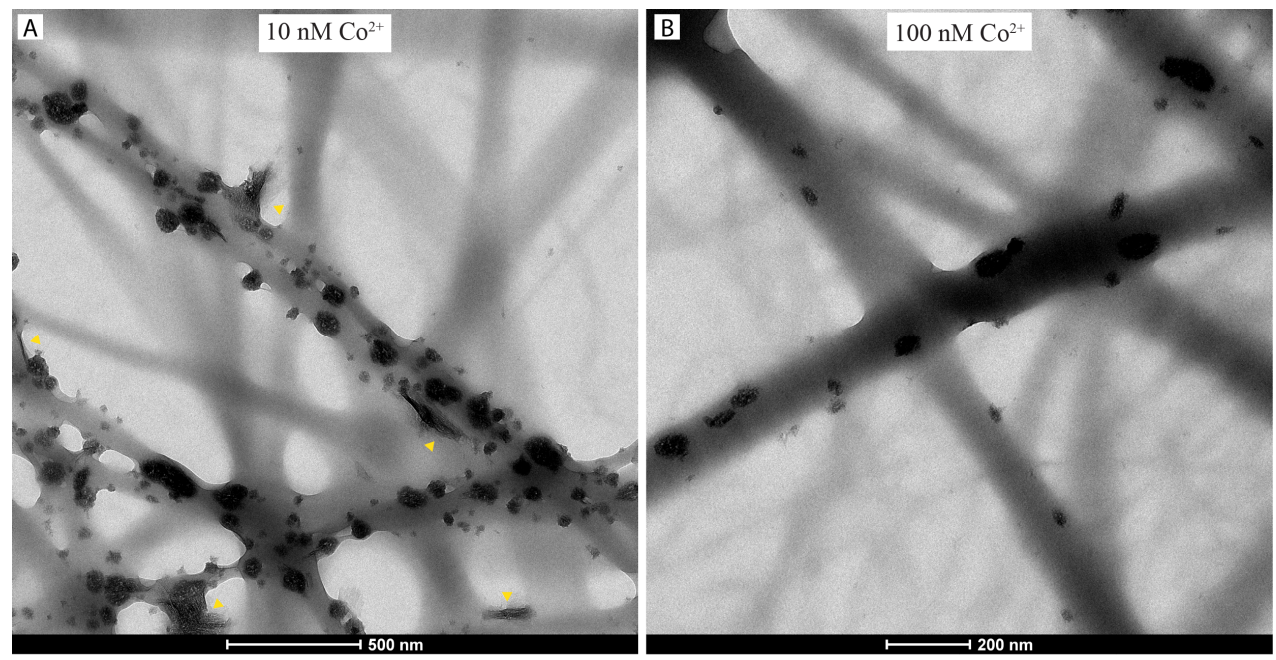

Figure 4. TEM images of collagen after immersion in a mineralization solution composed of the standard PILP formulation (50 mM Tris, $140 \mathrm{mM} \mathrm{NaCl}, 4.5 \mathrm{mM} \mathrm{CaCl}_{2}, 2.1 \mathrm{mM} \mathrm{K}_{2} \mathrm{HPO}_{4}$, $100 \mu \mathrm{g} / \mathrm{mL}$ pAsp) and an additional (A) 10 or (B) $100 \mathrm{nM} \mathrm{CoCl}$. Incubation was performed for $24 \mathrm{~h}$, at $37^{\circ} \mathrm{C}$, and $\mathrm{pH}=7.4$. Samples are unstained. Dense mineral aggregates (darker nodules) are visible in contact with collagen fibers. The majority of the nodules appear to have no ordered structure, with some exceptions where a striated pattern is visible (yellow arrowheads). Both types of mineral structures appear to be external or on the periphery of collagen fibers.

All three ions are present in bone apatite (see Chapter 2, subsection 1.3.2 Mineral Phase), and have been shown to influence bone regeneration processes in in vitro or in vivo studies. For example, magnesium has been shown to play an important role 
in bone metabolism and osteoblast proliferation in vitro. ${ }^{[23]}$ Strontium has a welldocumented beneficial effect on bone regeneration and bone remodeling. ${ }^{[24]}$ Cobalt, in addition to the effect on osteoclasts discussed in Chapter 4, has also been suggested to promote angiogenesis. ${ }^{[25,26]}$

Collagen fibers deposited on TEM grids were incubated in PILP mineralization solutions containing ions for $24 \mathrm{~h}$, after which the samples were air-dried overnight and imaged with TEM. Dense mineral aggregates (black) were observed close to collagen fibrils (dark grey), and appeared to have disordered structure. The addition of these cations, independently of concentration, prevented the formation of intrafibrillar mineral, presumably by disrupting the pAsp-stabilized amorphous calcium phosphate (ACP) aggregates or their interaction with collagen fibrils. The difficulty in including cations in intrafibrillar mineral has been described before, $[2,27]$ but the PILP method might be more amenable to anions, as was shown by a study where $\mathrm{F}^{-}$was added to the phosphate precursor solution without adverse effects on mineral formation. ${ }^{[28]}$ As discussed in Chapter 4, we circumvented this problem by incorporating $\mathrm{Co}^{2+}$ via a second $\mathrm{CaP}$ deposition step. 

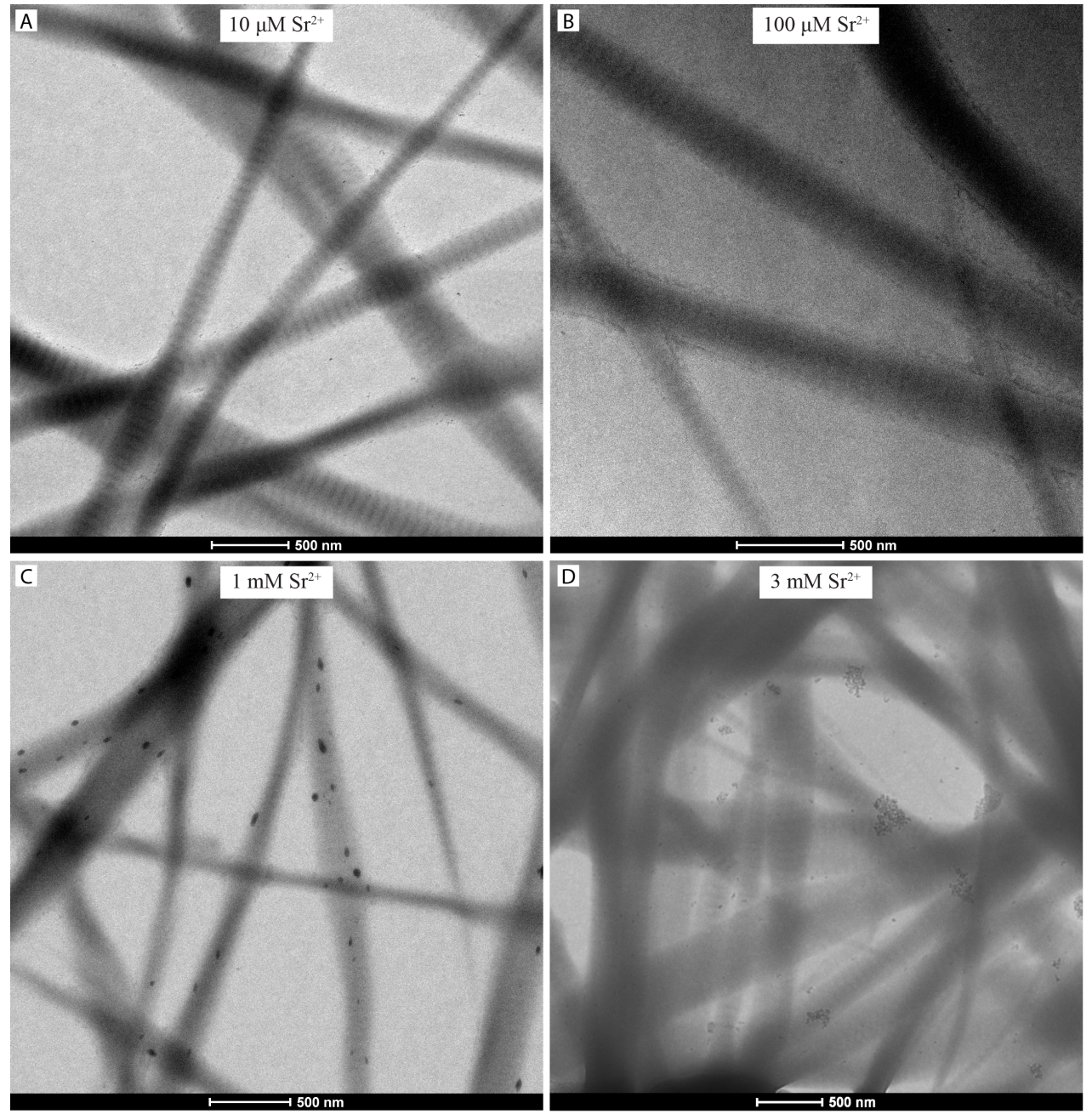

Figure 5. TEM images of collagen after immersion in a mineralization solution composed of the PILP formulation (50 mM Tris, $140 \mathrm{mM} \mathrm{NaCl}, 4.5 \mathrm{mM} \mathrm{CaCl}_{2}, 2.1 \mathrm{mM} \mathrm{K}_{2} \mathrm{HPO}_{4}, 100 \mu \mathrm{g} / \mathrm{mL}$ pAsp) and an additional (A) $10 \mu \mathrm{M}$, (B) $100 \mu \mathrm{M}$, (C) $1 \mathrm{mM}$ or (D) $3 \mathrm{mM} \mathrm{SrCl}$. Incubation was fperformed or $24 \mathrm{~h}$, at $37^{\circ} \mathrm{C}$, and $\mathrm{pH}=7.4$. Samples are unstained. At the lower concentrations (A) $10 \mu \mathrm{M}$ and (B) $100 \mu \mathrm{M}$, small aggregates of dense material can be seen as small dark dots close to collagen fibers in (A) and fuzzy outlines of collagen fibers in (B). At higher concentrations of $1 \mathrm{mM}(\mathrm{C})$ and $3 \mathrm{mM}(\mathrm{D})$, these aggregates are larger and easily observed next to collagen fibers. The majority of the mineral nodules have a disordered structure, and although always associated with collagen fibers, are located on their exterior. 

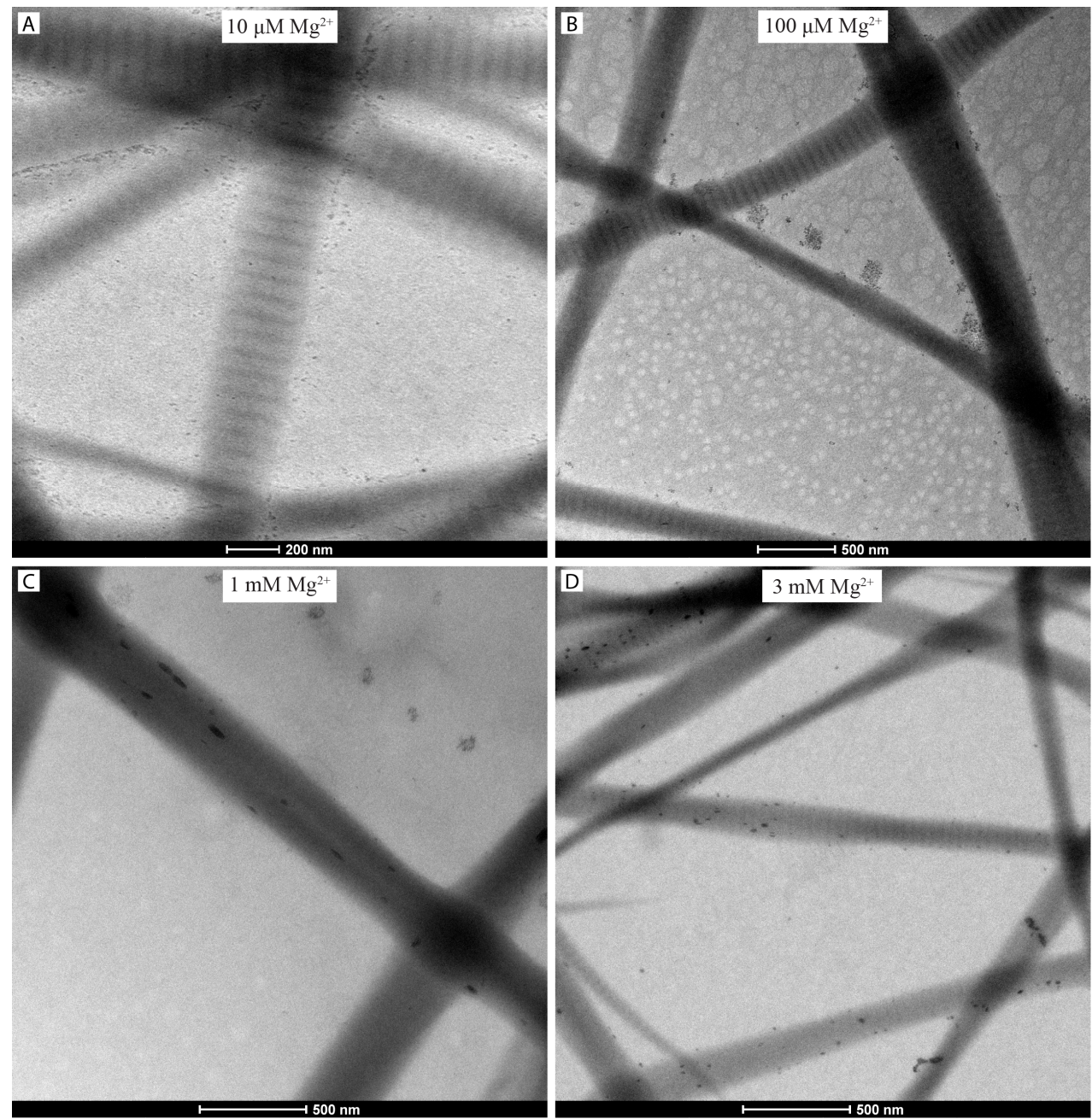

Figure 6. TEM images of collagen after immersion in a mineralization solution composed of the standard PILP formulation (50 mM Tris, $140 \mathrm{mM} \mathrm{NaCl}, 4.5 \mathrm{mM} \mathrm{CaCl}_{2}, 2.1 \mathrm{mM} \mathrm{K}_{2} \mathrm{HPO}_{4}$, $100 \mu \mathrm{g} / \mathrm{mL}$ pAsp) and an additional (A) $10 \mu \mathrm{M}$, (B) $100 \mu \mathrm{M},(\mathrm{C}) 1 \mathrm{mM}$ or (D) $3 \mathrm{mM} \mathrm{MgCl}$. Incubation was performed for $24 \mathrm{~h}$, at $37^{\circ} \mathrm{C}$, and $\mathrm{pH}=7.4$. Samples are unstained. In all conditions, small aggregates of dense material can be observed as darker objects next to collagen fibers. These disordered mineral nodules are predominantly found on the collagen fiber surface. 


\section{Flow cytometry analysis of efficiency of different commercial CD14+ monocyte isolation kits}

While the CD14+ monocytes used in the experiments described in the previous chapters were obtained as frozen aliquots (Lonza Bioscience, Switzerland), most parameters used in monocyte/macrophage cell culture and osteoclast differentiation were initially tested on monocytes isolated in-house from buffy coat samples obtained from the Dutch blood bank Sanquin. These included cell seeding density, cytokine concentration and supply regimen, and batch of serum. Based on cell morphology observations by light microscopy, we decided on $500 \mathrm{k}$ cells $/ \mathrm{cm}^{2}$; seeding with M-CSF (20 ng/mL) and culture 3-6 days before adding RANK-L (40 $\mathrm{ng} / \mathrm{mL}$ ) to the culture medium; and the specific batch of Fetal Calf Serum I from Hyclone (Fisher Scientific cat\# 10780245).

For the separation and selection of CD14+ monocytes used in the experiments described above, different commercial kits were tested. Here we present flow cytometry data collected for the frequency of CD14+ monocytes, before and after immunomagnetic selection from a population of mononuclear cells obtained from peripheral blood (PBMC) buffy coats by density gradient centrifugation (Figure 7). Three different immunomagnetic selection kits were compared for three different donors (Table 1).

Table 1. Summary of information about the immunomagnetic selection kits for CD14+ monocytes used in this experiment.

\begin{tabular}{lccc}
\cline { 2 - 4 } A & Type of selection & Manufacturer & Catalog number \\
\cline { 2 - 4 } B & Positive & StemCell Technologies & 17858 \\
C & Negative & StemCell Technologies & 19359 \\
& Positive & Miltenyi Biotec & $130-050-201$
\end{tabular}

Variability between donors is noticeable from the amount of CD14+ cells in the PBMC population before immunomagnetic selection (Table 2). By comparing the three kits for each donor, it was observed that the highest enrichment of CD14+ monocytes in the isolated cell population was achieved with kit $C$ (albeit with data from one donor only), followed by kit B and finally A (Table 2). Other differences influenced our choice of kit $B$ over kit $C$ as the one used for optimization of most parameters (cytokine concentration, cell density, FBS batch). The first was that the workflow was substantially easier and faster using kit $\mathrm{B}$, and the second the fact that it was a negative selection, which left the cells of interest unbound. Based on these 
results, we decided to use cells from a commercial supplier, in order to have cells from the same donor across the different studies.

Table 2. Summary of the flow cytometry results presented in Figure 7. Percentage of cell population that is $\mathrm{CD} 14+$ before (PBMC) and after immunomagnetic separation (Kits $\mathrm{A}, \mathrm{B}, \mathrm{C})$ for three different donors.

\begin{tabular}{lccc} 
& Donor 1 & Donor 2 & Donor 3 \\
\cline { 2 - 4 } PBMC & 17.4 & 19.8 & 13.0 \\
Kit A & 73.0 & 86.6 & 83.3 \\
Kit B & 76.3 & 92.5 & 87.8 \\
Kit C & 89.6 & &
\end{tabular}



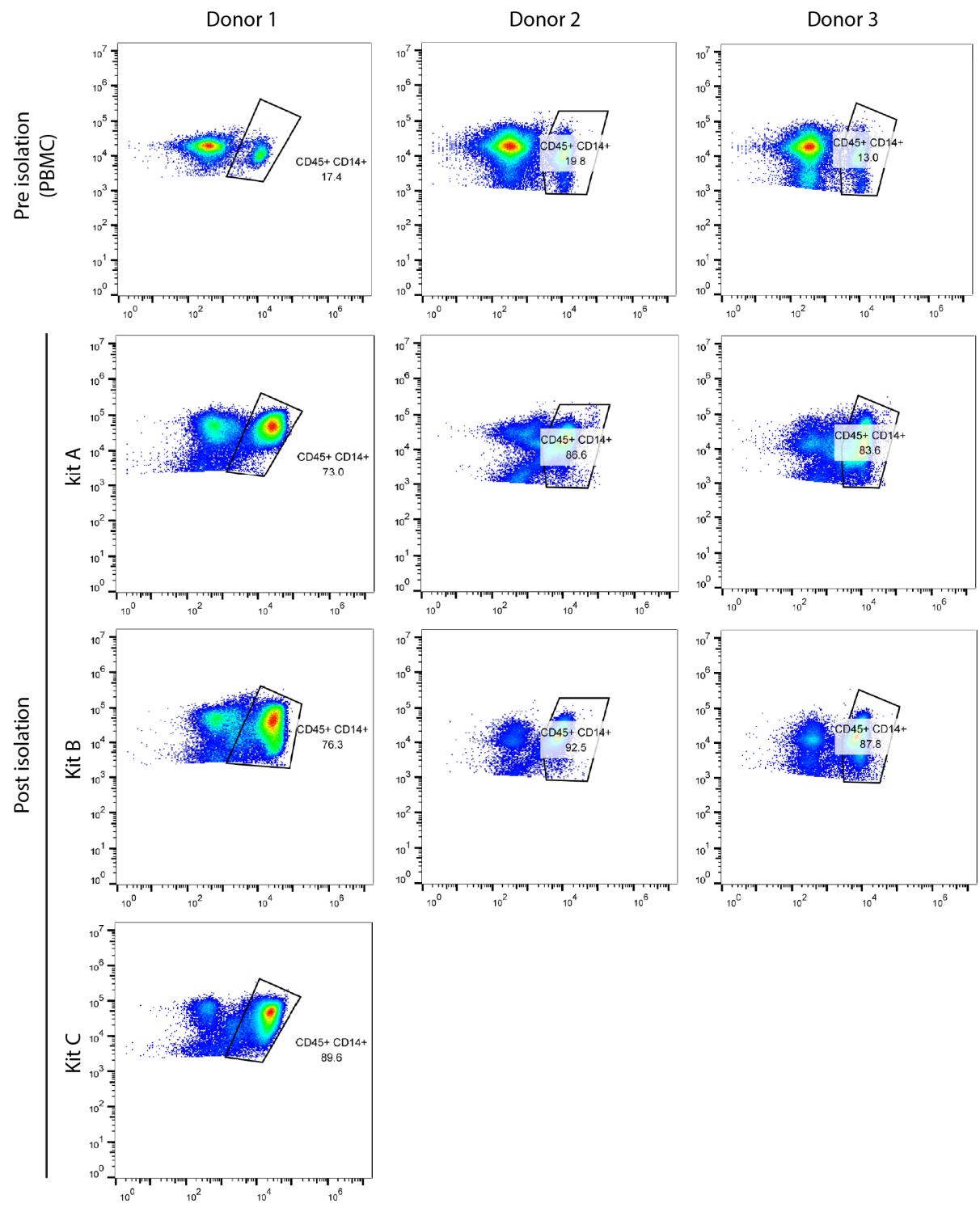

Figure 7. Flow cytometry data of peripheral blood mononuclear cells (PBMC) before and after immunomagnetic selection of CD14+ monocytes using different isolation kits. Cells were tagged with APC-conjugated CD45 (y-axis) and FITC-conjugated CD14 (x-axis) antibodies. The CD14+ population in the PBMC samples is between 10 and $20 \%$, and after selection of CD14+ cells with either of the three protocols/kits, becomes enriched to values between 70 and $95 \%$. 


\section{Osteoclasts differentiate from CD14+ monocytes in co-culture with U2OS osteosarcoma cells}

Preliminary osteoblast/osteoclast co-culture systems were explored as experimental setups that more closely resemble the in-vivo microenvironment surrounding an implant. Here, human osteosarcoma U2OS cells were placed in culture with CD14+ human monocytes derived from peripheral blood, without addition of cytokines. The experimental setup was initially defined as an indirect co-culture, with U2OS cells cultured separately from monocytes. Osteosarcoma cells were plated at $15 \mathrm{k}$ cells $/ \mathrm{cm}^{2}$ on 24-well plates and cultured for 14 days in basic medium, consisting of a-MEM supplemented with $10 \%$ FBS and antibiotics $(100 \mathrm{U} / \mathrm{mL}$ penicillin, 100 $\mu \mathrm{g} / \mathrm{mL}$ streptomycin). Meanwhile, monocytes were seeded at $500 \mathrm{k}$ cells $/ \mathrm{cm}^{2}$ on $96-$ well plates and cultured for 6 days in basic medium supplemented with $20 \mathrm{ng} / \mathrm{mL}$ $\mathrm{M}-\mathrm{CSF}$, to promote attachment. At subsequent monocyte media changes, medium from U2OS culture (U2OS-conditioned medium) was added to the monocyte/macrophage culture without additional cytokines. Although U2OS cells are adherent, some transfer of these cells to the wells with monocyte occurred during medium refreshments, because the medium was not filtered during the experiment. As a result, direct contact between the two cell types cannot be excluded. After 14 days in conditioned medium, cells were fixed and stained for TRAP and nuclei, and the amount of osteoclasts determined by image analysis. A control condition was used for comparison, where monocytes were cultured in basic medium supplemented with M-CSF (20 ng/mL) and RANK-L (40 ng/mL) for the same amount of time. This experiment was repeated with monocytes obtained from 3 different donors, each with 2 technical replicates (wells).

Large numbers of osteoclasts were observed after 14 days in the co-culture setup (Figure 8), more so than in the control with added cytokines (Table 3). We again observed a high variability in the numbers of osteoclasts among different donors, for both the co-culture and the control setup. For example, only one osteoclast was observed for donor 2, compared with a few tens of osteoclasts for the other two donors (condition with added cytokines). We must bear in mind that factors other than the donor characteristics could play a role too in the variations observed. For example, earlier medium refreshments can easily wash away loosely attached osteoclast precursors, hindering the chance of fusion later in the experiment. Depending on whether they were formed in co-culture or via stimulation with cytokines, osteoclast morphology was distinct. 


\section{Co-culture}
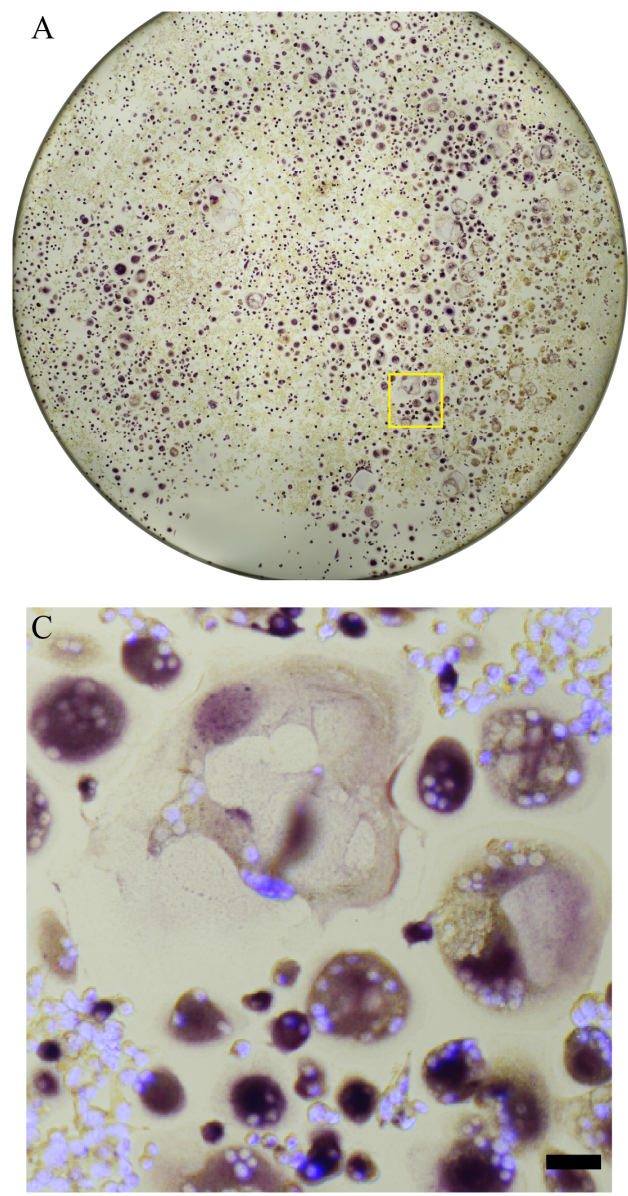

\section{Control}
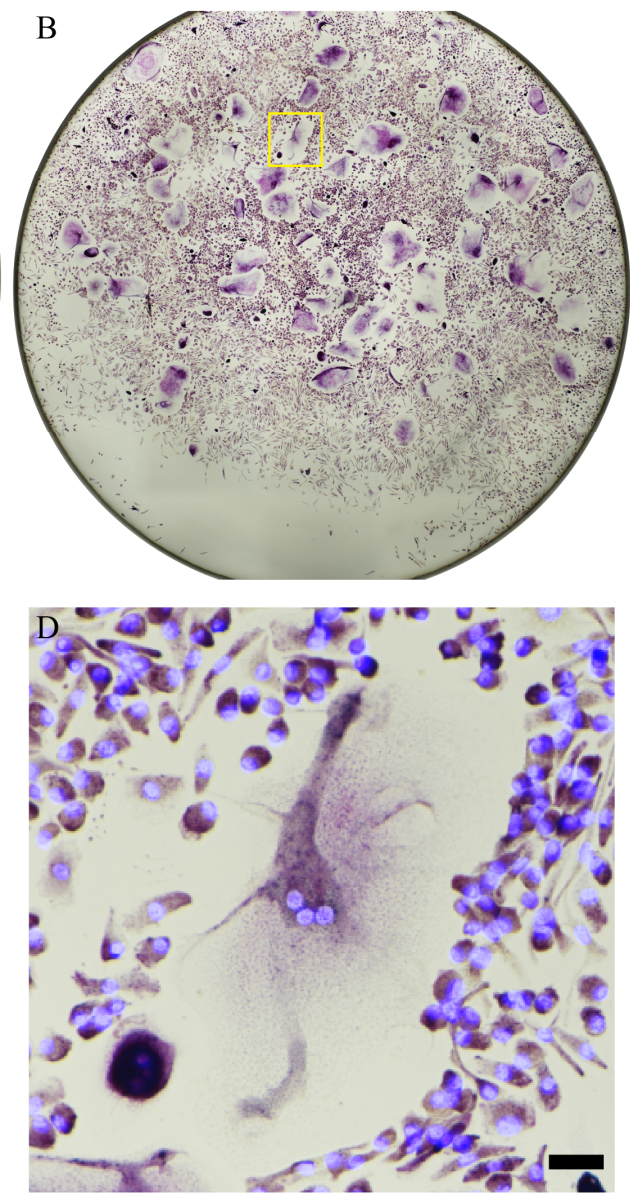

Figure 8. Brightfield and fluorescence (purple - TRAP, blue - DAPI) images of CD14+ monocytes after 14 days in co-culture with U2OS osteosarcoma cells (A, C). As control, CD14+ monocytes were cultured for the same amount of time in the presence of M-CSF $(20 \mathrm{ng} / \mathrm{mL})$ and RANK-L (40 ng/mL). Osteoclasts were observed in the co-culture system although no additional cytokines that stimulate osteoclastogenic differentiation were added.

Osteoclasts generated in co-culture were smaller, rounder and less spread than osteoclasts formed in the control condition (Figure 8). This could indicate differences in function, and further experiments are needed to verify, for example, their capacity for resorption. It is known from literature that U2OS cells secrete RANK-L, ${ }^{[29-33]}$ but to our knowledge the differentiation of osteoclasts in co-culture systems using the 
U2OS cell line has not been reported. This experiment showcases/illustrates a potential cytokine-free system to study the interplay of osteoblast-like cells, osteoclast formation and resorption of biomineralized collagen (and other) surfaces, as briefly mentioned at the end of Chapter 5 . Time constraints prevented further investigations using this co-culture system in the context of this thesis.

Table 3. Quantification of number of osteoclasts observed using image analysis of the entire well area per condition, donor, and technical replicate.

\begin{tabular}{cccc}
\hline U2OS medium & Donor 1 & Donor 2 & Donor 3 \\
\hline Well 1 & 481 & 96 & 135 \\
Well 2 & 340 & 78 & 228 \\
\hline Cytokine control & Donor 1 & Donor 2 & Donor 3 \\
\hline Well 1 & 10 & 0 & 15 \\
Well 2 & 27 & 1 & 49
\end{tabular}

\section{Acknowledgements}

Daniel Pereira would like to thank Lotte Strijbos for the contribution to Section 5. 


\section{References}

[1] M. J. Olszta, X. Cheng, S. S. Jee, R. Kumar, Y. Y. Kim, M. J. Kaufman, E. P. Douglas, L. B. Gower, Mater. Sci. Eng. R Reports 2007, 58, 77.

[2] F. Nudelman, P. H. H. Bomans, A. George, G. de With, N. A. J. M. Sommerdijk, Faraday Discuss. 2012, 159, 357.

[3] H. Nurrohman, K. M. M. Carneiro, J. Hellgeth, K. Saeki, S. J. Marshall, G. W. Marshall, S. Habelitz, PLoS One 2017, 12, 1.

[4] A. S. Deshpande, E. Beniash, Cryst. Growth Des. 2008, 8, 3084.

[5] S. Jee, L. Culver, Y. Li, E. P. Douglas, L. B. Gower, J. Cryst. Growth 2010, 312, 1249.

[6] S. Jee, T. T. Thula, L. B. Gower, Acta Biomater. 2010, 6, 3676.

[7] S. S. Jee, R. K. Kasinath, E. DiMasi, Y.-Y. Kim, L. Gower, CrystEngComm 2011, 13, 2077.

[8] T. T. Thula, F. Svedlund, D. E. Rodriguez, J. Podschun, L. Pendi, L. B. Gower, Polym. Basel 2011, 3, 10.

[9] Y. Li, T. T. Thula, S. Jee, S. L. Perkins, C. Aparicio, E. P. Douglas, L. B. Gower, Biomacromolecules 2011, 13, 49.

[10] F. Nudelman, K. Pieterse, A. George, P. H. H. Bomans, H. Friedrich, L. J. Brylka, P. A. J. Hilbers, G. De With, N. A. J. M. Sommerdijk, Nat. Mater. 2010, 9, 1004.

[11] A. J. Lausch, E. D. Sone, Biomacromolecules 2015, 16, 1938.

[12] D. Kim, B. Lee, S. Thomopoulos, Y.-S. Jun, Cryst. Growth Des. 2016, 16, 5359.

[13] T. T. Thula, D. E. Rodriguez, M. H. Lee, L. Pendi, J. Podschun, L. B. Gower, Acta Biomater. 2011, 7, 3158.

[14] A. K. Burwell, T. Thula-Mata, L. B. Gower, S. Habeliz, M. Kurylo, S. P. Ho, Y. C. Chien, J. Cheng, N. F. Cheng, S. A. Gansky, et al., PLoS One 2012, 7, DOI 10.1371/journal.pone.0038852.

[15] T. Thula-Mata, A. Burwell, L. B. Gower, S. Habelizt, G. Marshall, Mater. Res. Soc. Symp. Proc. 2011, 1355, 1.

[16] A. J. Lausch, B. D. Quan, J. W. Miklas, E. D. Sone, Adv. Funct. Mater. 2013, 23, 4906.

[17] H. Nurrohman, K. Saeki, K. Carneiro, Y. C. Chien, S. Djomehri, S. P. Ho, C. Qin, S. J. Marshall, L. B. Gower, G. W. Marshall, J. Mater. Res. 2016, 31, 321.

[18] L. S. Gu, J. Kim, Y. K. Kim, Y. Liu, S. H. Dickens, D. H. Pashley, J. Q. Ling, F. R. Tay, Dent. Mater. 2010, 26, 1077.

[19] Y. Liu, N. Li, Y. P. Qi, L. Dai, T. E. Bryan, J. Mao, D. H. Pashley, F. R. Tay, Adv. Mater. 2011, 23, 975.

[20] F. R. Tay, D. H. Pashley, Biomaterials 2008, 29, 1127.

[21] D. Geblinger, C. Zink, N. D. Spencer, L. Addadi, B. Geiger, J. R. Soc. Interface 2012, 9, 1599.

[22] Z. Tahmasebi Birgani, A. Malhotra, L. Yang, B. Harink, P. Habibovic, in (Ed: P. B. T.C. B. I. I. Ducheyne), Elsevier, Oxford, 2017, pp. 406-427.

[23] S. Lala, M. Ghosh, P. K. Das, D. Das, T. Kar, S. K. Pradhan, Mater. Chem. Phys. 2016, 170, 319 .

[24] N. Neves, D. Linhares, G. Costa, C. C. Ribeiro, Bone Jt. J. 2017, 6, 366.

[25] Z. T. Birgani, E. Fennema, M. J. Gijbels, J. De Boer, C. A. Van Blitterswijk, P. Habibovic, 
Acta Biomater. 2016, 36, 267.

[26] Z. T. Birgani, N. Gharraee, A. Malhotra, C. A. Van Blitterswijk, P. Habibovic, Biomed. Mater. 2016, 11, DOI 10.1088/1748-6041/11/1/015020.

[27] L. B. Gower, in Biominer. Biomater. Fundam. Appl., 2015.

[28] H. Nurrohman, S. Habelitz, G. W. Marshall, L. B. Gower, Dent. Mater. 2018, 34, 1378.

[29] K. Kinpara, M. Mogi, M. Kuzushima, A. Togari, J. Immunoassay 2000, 21.

[30] L. Mancini, M. J. Paul-clark, G. Rosignoli, R. Hannon, J. E. Martin, I. Macintyre, M. Perretti, Am. J. Pathol. 2007, 170, 1018.

[31] S. Chen, F. Huang, S. Lee, M. Li, Y. Chang, Int. Endod. J. 2009, 42, 375.

[32] M. Mogi, A. Kondo, J. Immunoass. Immunochem. 2013, 34, 356.

[33] F. M. Huang, S. S. Lee, S. F. Yang, Y. . Chang, J. Endod. 2019, 35, 363. 
Chapter 7

General discussion 


\section{Incorporating biomineralized collagen in the bone remodeling process - toward effective bone graft substitutes}

In this thesis, we set out to investigate biomineralized collagen as a potential biomimetic material for use as a bone graft substitute, specifically by exploring its interactions with osteoclasts and mesenchymal stromal cells, two main cell types relevant to remodeling and regeneration of bone. In particular, the focus on osteoclasts stems from their resorptive role in the process of bone remodeling, as outlined in Chapter 1. To briefly recall, in the bone remodeling cycle, the process of bone removal by osteoclasts is coupled by various communication mechanisms to the process of bone deposition by osteoblasts. Hence, the question whether a biomineralized collagen surface, which, from a chemical and structural perspective closely resembles natural bone tissue, can be resorbed by osteoclasts, bears importance in the context of its possible applications as a bone graft substitute material. The interest in biomineralized collagen itself is indeed rooted in its composition and hierarchical organization at the sub-micron scale, which represent a step forward in bone mimicking materials when compared to other available composite materials, which are often much simpler mixtures of the organic and inorganic components. The extent to which biomineralized collagen, used in our studies as a flat membrane, mimics bone tissue, is discussed at length in Chapter 2, but a brief summary is made here before discussing final considerations regarding the experimental results obtained. To better understand/visualize what makes biomineralized collagen similar to the native bone (and where it falls short), it is useful to think in terms of scale, from smaller (sub-micron scale) to larger (millimeter scale) structures. The biomimetic aspect of biomineralized collagen is most obvious at the smaller end of the scale, below $1 \mu \mathrm{m}$. Specifically, the arrangement of its two main components, fibrillary type I collagen and nanoscale hydroxyapatite particles, in which the mineral is predominantly found inside the collagen fibrils, oriented along the direction of the fibril, is representative of the native tissue. Nevertheless, at this sub-micron scale, some differences from the native tissue are already recognized. The two major differences are the absence of non-collagenous proteins, and the chemical composition of the apatite phase, which lacks the highly substituted character of bone apatite. Increasing the length scale up to hundreds of microns, in bone we find organized structures (osteons) composed of fibril arrays, packed densely around hollow spaces (Haversian and Volkmann canals, lacunae, 
canaliculi) where blood, nerves and cells reside. This level of architectural organization is obviously absent in our membranes, and so are the biological components, but perhaps more importantly, the density of the fibrous matrix, i.e., the number of mineralized fibrils in a certain volume unit, is lower than that of extracellular matrix (ECM) in secondary bone, where osteons are found. In the membranes used in our studies, collagen fibers are more loosely packed, and the space between them is occupied by water. Mechanical behavior at the macro-scale is affected by this difference in density, and it was shown in Chapter 4 that stiffness, determined by nano-indentation measurements, was closer to that of a gel (1-10 $\mathrm{MPa})$, than to the values normally reported for cortical bone (10-25 GPa). ${ }^{[1]}$ This point will be discussed again in our final considerations regarding biomimetism as tool for material engineering and design.

The main conclusion from the studies presented in this thesis is that resorption of biomineralized collagen by osteoclasts is possible. The extent to which resorption takes place, however, seems to depend on several physical and chemical characteristics of the materials. We hypothesize that some of the physical characteristics that influenced resorption are surface structure (topography), porosity and mechanical properties. On the chemical composition side, our results suggest that nothing inherent to the chemical nature of biomineralized collagen is an obstacle for resorption, but instead that the extent to which resorption takes place can be influenced by specific chemical composition details, such as the presence of RGD ligands and bioactive ions. We will first go through the results that sustain these hypotheses, followed by the shortcomings of our studies that take away some certainty about the conclusions drawn from them.

As is described in Chapter 4, a collection of experimental results points to (limited) resorption events taking place on the CoCaP-coated biomineralized collagen membranes. These were fluorescence images of cells showing F-actin structures resembling actin rings, SEM images of cavities in the surface of membranes (after cell removal) that matched in size with the actin rings, and increased $\mathrm{Ca}^{2+}$ concentration in cell culture medium measured by ICP-MS. The formation of actin rings, resorption cavities and increased $\mathrm{Ca}^{2+}$ concentration in medium were not observed on the uncoated biomineralized collagen membranes used in Chapters 3 
and 4, which led us to conclude that the modification of the substrate with a cobaltcontaining coating facilitated osteoclast resorption. One major shortcoming of this study was the lack of an additional control containing only one of the two modifications introduced by the coating step. This led to the main point of discussion in Chapter 4 being whether the inclusion of cobalt or the extra mineral amount, (shown to increase the substrate stiffness from 1 to $10 \mathrm{MPa}$ ) was the underlying cause of the observed resorption events. Nevertheless, the inability to pinpoint the underlying cause does not detract from the observed effect, i.e., the fact that resorption actually occurred, which is evidence that there is no obstacle to resorption inherent to the chemical nature of biomineralized collagen. One could argue that the original chemical composition of the biomineralized collagen was modified by the addition of cobalt in the coating solution. However, there is no evidence in literature that suggests that cobalt triggers the resorption of a material that was previously not able to undergo resorption. The literature evidence for an effect of cobalt on osteoclast resorption at the concentrations we found (around $7 \mathrm{nM}$ in culture medium after 2-3 days) is scarce and conflicting, pointing to either no effect, or a small positive or negative effect on resorption (Patntirapong 2009; Andrews 2011). So it is safe to assume that even if cobalt played a role in the resorption events observed in Chapter 4, (cobalt-free) biomineralized collagen can probably undergo osteoclast resorption too. Nevertheless, this statement is not supported by the results of Chapter 3, where we were not able to detect resorption of the original biomineralized membranes. What was the reason for the lack of resorption? As is discussed in Chapter 3, the two main factors hypothesized to have prevented resorption are the lack of RGD ligands to the vitronectin receptor (integrin $\left.\alpha v \beta_{3}\right)$ and physical characteristics of the substrate. Based on literature, we know (1) that actin ring formation and resorption are dependent on the pore size of the substrate with sizes in the range 1 to $3 \mu \mathrm{m}$ inhibiting these events on $\beta$-TCP ceramics, ${ }^{[2]}$ and (2) that osteoclasts have the ability to form actin rings on soft materials (30 to $1800 \mathrm{kPa}$ ). ${ }^{[3]}$ This suggests that a major barrier for actin ring formation (and subsequent resorption) in our study was the pore size associated with the mesh-like surface structure of the membrane. However, as was shown in Chapter 4, the surface structure was unaffected by the CoCaP coating step, presenting the same pore size and fibrillary structure. On the other hand, the coating process increased the stiffness of the membranes from 1 to $10 \mathrm{MPa}$. This change 
appears to have facilitated the ability of osteoclasts to form actin rings, counteracting the pore size obstacle at least to some extent. Taken together, these results indicated that the physical characteristics of the substrate used did have an effect on the ability of osteoclasts to seal an area for resorption. However, actin ring formation cannot be predicted simply based on either characteristic (i.e., pore size smaller than $1 \mu \mathrm{m}$ or stiffness higher than $1 \mathrm{MPa}$ ) as these two variables are interdepend. In the case of our biomineralized collagen membranes, decreasing the pore size by increasing the density of fibers (which in turn also increases the stiffness of the substrate) would likely result in a surface more amenable to osteoclast resorption. As recapitulated at the start of this chapter, density of fibers in our membranes is one property that makes them significantly different from native bone. This property is now recognized as a key feature of the ECM that should be replicated to allow normal osteoclast behavior. This aspect will be addressed again at the end of this chapter, when discussing the future of biomineralized collagen as a biomimetic material for bone regeneration.

Now moving the focus to another chemical characteristic of our biomineralized collagen membranes that could have influenced the ability of osteoclasts to form actin rings and resorb the substrate: the presence of integrin-binding motifs. We have mentioned the lack of RGD ligands to the vitronectin receptor in Chapter 3 as a possible cause of inhibition of actin ring formation and resorption. Integrin $\alpha v \beta_{3}$ is the most abundantly expressed integrin in osteoclasts, ${ }^{[4]}$ and its RGD ligands are present in vitronectin, osteopontin, bone sialoprotein, fibronectin and von Willebrand factor. ${ }^{[5,6]}$ Integrin $\alpha_{v} \beta_{3}$ is recognized for playing a crucial role in osteoclast attachment and resorption.[7] Knock-out studies in mice ( $\beta_{3}$ deletion) resulted in osteopetrosis, a disease caused by significant inhibition of osteoclast resorption. ${ }^{[8,9]}$ However, osteoclastogenesis appeared to be unaffected. ${ }^{[9]}$ Targeting of integrin $\alpha_{v} \beta_{3}$ with peptides, antibodies ${ }^{[10,11]}$ or integrin-disrupting drugs ${ }^{[12,13]}$ resulted in significantly reduced osteoclast resorption in vitro. There is mention of integrin $\alpha_{v} \beta_{3}$ being able to interact with the cryptic RGD site in collagen, but only after collagen denaturation, ${ }^{[14,15]}$ which means that there is no ligand for integrin $\alpha v \beta_{3}$ in our membranes. However, the calf serum used in cell culture medium could provide proteins that contain such ligands. Another integrin present in osteoclasts, $\alpha_{2} \beta_{1}$ is responsible for interacting with collagen ${ }^{[16,17]}$ and collagen alone is sufficient 
to allow osteoclast attachment and actin ring formation, even in the absence of serum proteins. ${ }^{[18]}$ Our results on osteoclast formation on collagen membranes shown in Chapter 3 and the literature discussed above demonstrate that the attachment of osteoclasts to collagen or biomineralized collagen is possible, probably via integrin $\alpha_{2} \beta_{1}$. However, their resorptive activity can be inhibited to a great extent if there is no activation of integrin $\alpha_{v} \beta_{3}$, although this could be partially countered by proteins carried by the serum used for cell culture. In conclusion, substrate abundance of RGD ligands to both integrin $\alpha v \beta_{3}$ and $\alpha_{2} \beta_{1}$ modulates the extent to which resorption takes place. Referring back to our initial considerations regarding the biomimetic character of the biomineralized collagen used in our studies, this is another example of where it falls short of the native tissue: the non-collagenous proteins found in bone have no analogue in biomineralized collagen which in turn results in the lack of RGD ligands to the vitronectin receptor.

Summarizing the discussion points so far, we have shown that biomineralized collagen can be resorbed by human osteoclasts, as was demonstrated by the case of the CoCaP-coated membranes in Chapter 4. We have argued that physical characteristics of the material, including surface structure, porosity and stiffness can completely inhibit resorption, and that these were likely the main reason why no resorption events were observed in Chapter 3 and on the uncoated membranes of Chapter 4. We have also argued that chemical characteristics of biomineralized collagen are likely not an obstacle to resorptive function, because resorption events were observed with minimal modification of the original biomineralized membranes. However, characteristics such as abundance of RGD ligands to integrin $\alpha v \beta_{3}$, or presence of bioactive ions such as $\mathrm{Co}^{2+}$ can modulate cell activity and ultimately the extent to which resorption actually occurs. The physical and chemical properties of biomineralized collagen can be further tuned to bring them closer to the properties of native tissue, a topic we will return to in the last part of this discussion.

Resorption of biomineralized collagen by osteoclasts is interesting because, if it occurs in vivo, it is expected to be followed by the process of bone deposition by osteoblasts. This entails communication between osteoclasts and osteoblast precursors, which are triggered to differentiate and produce ECM. Osteogenic 
differentiation can also be triggered by biomaterial itself, an aspect we explored in Chapter 5 where we looked into expression of genes commonly associated with osteogenic differentiation by human mesenchymal stromal cells (hMSCs) cultured on biomineralized collagen membranes. The results showed some indication of an osteogenic effect of the substrate on hMSCs, with elevated mRNA levels of RUNX2, SPP1, ENPP1, and OCN after 3 days, and COL1A1 after 14 days of culture on biomineralized collagen. Rather than focusing on the intrinsic ability of the biomaterial to trigger the differentiation of undifferentiated cells into the osteogenic lineage, a phenomenon also known as osteoinduction, ${ }^{[19,20]}$ we were interested in the idea of incorporating a biomaterial in the physiological cycle of bone remodeling. To explore this idea, it is important to analyze whether osteoclastic resorption (in vitro) is indeed followed by osteogenic differentiation of osteoblast precursors, (triggered by communication with osteoclasts) and, eventually, new bone matrix deposition. Co-culture systems of cells can be useful in the context of replicating the bone remodeling process and understanding communication between osteoclasts and osteoblasts or their precursors. This communication takes different forms and is governed by different mechanisms, as recently reviewed.[21] Osteoclasts can secrete factors and vesicles, express membrane proteins, and release matrix-bound factors that influence the osteogenic lineage. Even in the absence of resorption, conditioned medium from pre-differentiated osteoclasts was shown to induce osteogenic differentiation in hMSCs, as was evidenced by increased ALP activity and matrix mineralization. ${ }^{[22]}$ The experimental setup used in that study could be adapted to include biomaterials, for example, by collecting medium from actively resorbing osteoclasts on a biomaterial surface and comparing its effects on hMSCs differentiation or osteoblast function to the effects exerted by conditioned medium from osteoclast culture on bone surfaces. In Chapter 5 no co-culture setup was used, as the focus was on analyzing the osteogenic gene expression of hMSCs driven by biomineralized collagen. However, osteoclast-related genes were also investigated, finding a low RANK-L/OPG ratio, suggestive of a resorption-inhibiting phenotype. As more knowledge is obtained regarding osteoclast-osteoblast communication, we will be able to better characterize the interplay between osteoclasts, biomaterials and the osteoblast lineage, and know whether a biomaterial can indeed trigger the same type of communication that results in bone deposition in physiological conditions. For an example pilot study, we refer to Chapter 6 where we describe a co-culture 
system based on human monocytes and osteoblast-like cell line U2OS. In this context, coupling factors are interesting, such as the apoptotic bodies from mature osteoclasts, which were shown to trigger osteogenic differentiation via reverse RANK-L signaling, as well as enhance bone formation when implanted in a bone defect in mice. ${ }^{[23]}$

\section{Future perspectives - fine tuning properties and function of biomineralized collagen}

Based on the discussion above, it has become clear that the biomineralized collagen membranes used for the studies in this thesis resembled natural bone ECM to a certain extent, but also showed important differences in terms of composition (lack of biochemical cues provided by non-collagenous proteins and lack of trace ions in the inorganic phase) as well as physical characteristics (density and organization of mineralized fibers and, ultimately, mechanical properties). We concluded that the mesh-like surface structure of the membrane, and the consequent overall low volume density of mineralized fibers, was the main inhibiting factor of resorption by osteoclasts in Chapter 3. We also posited that the increase in stiffness reported in Chapter 4 was the main reason behind the resorption events observed. Increasing the density of mineralized fibers would reduce the porosity and pore size, and the overall water content. The surface of the membranes would thus be smoother and less porous, and the material would be stiffer. These changes would likely improve the capacity for osteoclasts to resorb this material.

Increasing the density of highly hydrated gels has been a thorn in the side of biomaterials scientists for some time. Two conceptually different methods exist to increase density of biomineralized collagen membranes. The first is used after the formation of the hydrated gel, before cross-linking and mineralization take place, and results in reduction of the initial water content between collagen fibers. There are two main techniques capable of achieving this effect. The first one is Plastic Compression (PC), which uses compression to remove water uni-directionally through a mesh. ${ }^{[24]}$ This technique is similar to the one we applied to prepare collagen membranes, but includes adding weight to apply compression, in contrast to our method where water was removed from the material through a nylon mesh by gravity alone. The second technique is Gel Aspiration-Ejection (GAE), which uses 
pressure differentials to aspirate a pre-formed hydrated gel into a syringe of specific shape and surface texture, resulting in expulsion of excess water and alignment of fibers to some extent. ${ }^{[25]}$ The alternative approach to increasing biomineralized collagen membrane density is to do it after the process of intrafibrillar mineralization, by replacing most of the water in pores with additional mineral. The method is similar to the one used in Chapter 4, but with substantially more mineral added, by increasing the concentration or the volume of mineralization solution. From a biomimetic perspective, too much extrafibrillar mineral is not desirable, as most of bone apatite is found inside the collagen fibers, and therefore, the first method is preferred. However, using an extra mineralization step allows for the inclusion of bioactive ions (which is difficult if not impossible in intrafibrillar mineralization), as shown in Chapter 4 , which is desirable not only from a perspective of mimicking the apatite in natural bone, but also because bioactive ions per se can have an effect on osteoclast activity and osteogenic differentiation.

Mimicking the architectural organization found in bone at the scale of hundreds of microns is another yet unresolved challenge. The precise architecture of cavities/channels found in bone is probably too difficult to replicate at the moment. However, their function as communication spaces where blood, nutrients, and cells can move in an otherwise prohibitively dense material is crucial. A hypothetical three-dimensional construct composed of biomineralized collagen needs to replicate this function in order to act as a bone graft substitute. Importantly, such a scaffolds needs to enable blood and host cells to interact over the entire volume of the construct, instead only through its surface. In Chapter 2 we discussed different approaches that could be used to achieve a dense 3D biomineralized collagen constructs incorporating such communication channels, wet spinning, microfluidic extrusion and reverse dialysis being a few examples of these approaches.

The rationale for exploring biomineralized collagen as a possible material for bone graft substitutes is rooted in its biomimetic characteristics, which, although not perfect yet, give it a certain attractiveness, not only from a functionality perspective, but also from a perspective of acceptance by the end users (clinicians and patients). There are, however, also other potential applications for this material. In-vitro bone models that aim to provide a micro environment close to the native tissue could 
make use of a material like biomineralized collagen. This was exemplified by a study where hMSCs and either vascular or neural cells were co-embedded in a biomineralized collagen construct, showing that this biomimetic material supports complex biological models.[26] Biomaterials used in bone disease or drug testing studies are often collagen, $\mathrm{CaP}$, or a combination of both, and have followed a trend of increasing biomimicry. ${ }^{[27]}$ 3D-printed scaffolds used in studies of bone metastatic cancer are made up of polymers such as poly( $\varepsilon$-caprolactone) or poly(lactic acid), with or without HA particles.[28] Bioprinted scaffolds usually consist of a hydrogel with embedded cells, sometimes with HA added to the mix as well. ${ }^{[29]}$ Microfluidic and organ-on-a-chip designs, either for bone, ${ }^{[30]}$ or bone marrow, ${ }^{[31]}$ also employ hydrogels, polymers, CaPs, or their composites to reproduce the ECM of bone. ${ }^{[32]}$ The materials described above for disease modelling and microenvironment replication do not have the characteristic collagen-apatite hierarchical organization at the sub-micron scale that biomineralized collagen and bone have. The physiological relevance of in vitro studies that rely on microenvironment replication is expected to increase as the level of biomimicry of these artificial microenvironments draws closer to their natural analogues, and this is exactly what biomineralized collagen offers for bone tissue.

\section{Concluding thoughts about importance of biomimetism in biomaterials design}

In the final part of this discussion, it is important to critically evaluate biomimetism as an inspiration and driver of materials design for biomedical applications, including regenerative medicine. Biomimicry as an engineering approach is a logical derivative from knowledge of the complex structures, processes, and functions that nature has developed over the course of 3.7 billion of years of evolution - nature's design as a gold standard. More specifically, application of biomimetic approaches to bone regenerative medicine is widespread and has yielded immense advances, both clinically, with the continued development of synthetic materials that have biomimetic characteristics (e.g., over $20 \mathrm{CaP}$-based bone graft substitutes are commercially/clinically available in the USA $\left.{ }^{[33]}\right)$, as well as in pre-clinical studies, as a way to replicate the physiological microenvironement in vitro and learn about fundamental processes from nature. 
Despite the advances achieved so far, it is important to ask the question whether biomimetism is the best engineering approach for biomaterials in general and bone graft substitutes in particular. This thesis, as well as work by others on biomineralized collagen, has shown that making a perfect mimic of a natural material is not a trivial task, while the biological function seems to be little forgiving of imperfections in the design. In other words, the question is whether we should aim for perfection in biomimetic design and development, in the hope to optimize the function, or aim for the optimal function by using approaches other than biomimetism.

Indeed, instead of design strategies based on a pre-existing concept, it is possible to approach the problem in the completely opposite way, eschewing any preformed idea (prejudice) over composition and physical characteristics. This can be done by producing and screening large libraries of materials and properties for those that maximize a certain outcome, for example trigger of osteogenic differentiation, antiinflammatory response, resorptive activity, etc. Advantages of this strategy, that is analogous to discovery approaches in the field of pharmacology, are that it considers materials and parameters that would normally not be considered when following a biomimetic approach (e.g., the use of metals in bone or use of highly degradable materials that do not mimic bone tissue, but do trigger rapid regeneration of natural tissue). It is however difficult to build such libraries in the first place, due to the multitude of approaches and differences in experimental design needed for different material types and also because parametrization of properties is difficult and often limited to a few examples.

The use of magnesium-based alloys as bone graft substitutes is an example of a nonbiomimetic material performing well as bone graft substitute, having better mechanical compatibility with bone than ceramic-only solutions, while still being osteoconductive and degrading in the physiological environment. ${ }^{[34]}$ Conductive polymers also exhibit little resemblance with the ECM of bone, but have nonetheless shown promising results in pre-clinical studies, offering the possibility of being combined with external electrical stimulation. ${ }^{[35]}$

The examples above illustrate the expanded possibilities when looking beyond biomimetics, although they also have inherent drawbacks, such as difficult to control corrosion/degradation in the case of magnesium alloys, ${ }^{[36]}$ and poor mechanical properties in the case of conductive polymers. 
Having a blueprint (the native tissue) offers a clear engineering goal. It does come with its own drawbacks, the main one being the sheer complexity of the native tissue, making it very difficult to know which of its characteristics are critical and require attention in order to design and develop a functional biomaterial. Thinking of bone especially, it is important to emphasize that the majority of the now available CaP-based bone graft substitutes, which have shown enormous clinical successes, are comparatively much simpler than the ECM of bone. They do however, replicate key characteristics of bone tissue, which are sufficient to make them, e.g., osteoconductive or even osteoinductive. Nevertheless, these key characteristics are hard to decipher a priori, and must be tested. For example, in our own experiments of osteoclast resorption, the biomineralized collagen membrane showed more bone biomimetic components than, e.g., a microporous beta-TCP surface. However, osteoclasts could readily resorb the beta-TCP surface, unlike the biomineralized collagen membrane. We arrive seemingly at a paradox, where a more biomimetic material (in design) actually shows less biomimetic characteristics (in function) than other synthetic, less mimetic materials. One conclusion that can be drawn immediately is that a biomimetic design does not guarantee improved performance, or in other words, does not guarantee a "biomimetic function". Another may be that the assumption of biomineralized collagen as the "more biomimetic" may be too simplistic or irrelevant, as it is solely based on bone-mimicking composition and structure. The hallmark of a truly biomimetic material is to be recognized by host cells as indistinguishable from the native tissue. This is only possible if the key characteristics of the original tissue are replicated in a synthetic biomaterial. Studies where biomimicry is used in novel biomaterials and focus on cell-material interaction give valuable information that can be used not only in designing better materials, but also in deepening our understanding of the native tissue. This is one of the advantages of biomimetics, that in striving for replicating properties of the native tissue, insight can be gained about the native tissue itself. 


\section{References}

[1] M. Casanova, A. Balmelli, D. Carnelli, D. Courty, P. Schneider, R. Müller, R. Soc. Open Sci. 2017, 4, 160971.

[2] N. L. Davison, X. Luo, T. Schoenmaker, V. Everts, H. Yuan, F. Barrère-de Groot, J. D. de Bruijn, Eur. Cells Mater. 2014, 27, 281.

[3] H. Touaitahuata, E. Planus, C. Albiges-Rizo, A. Blangy, G. Pawlak, Eur. J. Cell Biol. 2013, 92, 139.

[4] J. Clover, R. A. Dodds, M. Gowen, J. Cell Sci. 1992, 103 ( Pt 1, 267.

[5] N. Rucci, A. Teti, Matrix Biol. 2016, 52-54, 176.

[6] W. Zou, S. L. Teitelbaum, 2010, 1192, 27.

[7] I. Nakamura, L. T. Duong, S. B. Rodan, G. A. Rodan, 2007, 337.

[8] W. Zou, S. L. Teitelbaum, J. Cell Biol. 2015, 208, 125.

[9] K. P. McHugh, K. Hodivala-Dilke, M. H. Zheng, N. Namba, L. Jonathan, D. Novack, X. Feng, F. P. Ross, R. O. Hynes, S. L. Teitelbaum, J. Clin. Invest. 2000, 105, 433.

[10] T. J. Chambers, K. Fuller, J. A. Darby, J. A. Pringle, M. A. Horton, Bone Miner. 1986, $1,127$.

[11] M. A. Horton, M. L. Taylor, T. R. Arnett, M. H. Helfrich, Exp. Cell Res. 1991, 195, 368.

[12] M. Sato, M. K. Sardana, W. A. Grasser, V. M. Garsky, J. M. Murray, R. J. Gould, J. Cell Biol. 1990, 111, 1713.

[13] J. E. Fisher, M. P. Caulfield, M. Sato, H. A. Quartuccio, R. J. Gould, V. M. Garsky, G. A. Rodan, M. Rosenblatt, Endocrinology 1993, 132, 1411.

[14] G. E. Davis, Biochem. Biophys. Res. Commun. 1992, 182, 1025.

[15] M. H. Helfrich, S. A. Nesbitt, P. T. Lakkakorpi, M. J. Barnes, S. C. Bodary, G. Shankar, W. T. Mason, D. L. Mendrick, H. K. Väänänen, M. A. Horton, Bone 1996, $19,317$.

[16] M. H. Helfrich, S. A. Nesbitt, E. L. Dorey, M. A. Horton, J. bone Miner. Res. Off. J. Am. Soc. Bone Miner. Res. 1992, 7, 335.

[17] P. A. Townsend, I. Villanova, A. Teti, M. A. Horton, Eur. J. Cell Biol. 1999, 78, 485.

[18] I. Nakamura, N. Takahashi, T. Sasaki, E. Jimi, T. Kurokawa, T. Suda, J. Bone Miner. Res. 1996, 11, 1873.

[19] M. Bohner, R. J. Miron, Mater. Today 2019, 22, 132.

[20] E. García-gareta, M. J. Coathup, G. W. Blunn, Bone 2015, 81, 112.

[21] N. A. Sims, T. J. Martin, Annu. Rev. Physiol. 2020, 82, 507.

[22] T. Stessuk, J. Husch, I. At, S. Hofmann, J. Jjp, V. Den Beucken, J. Immunol. Regen. Med. 2021, 12, 100044.

[23] Q. Ma, M. Liang, Y. Wu, F. Luo, Z. Ma, S. Dong, J. Xu, C. Dou, Bone Res. 2021, 9, 5.

[24] U. Cheema, R. A. Brown, Adv. wound care 2013, 2, 176.

[25] G. Griffanti, E. Rezabeigi, J. Li, M. Murshed, S. N. Nazhat, 2019, 1903874, 1.

[26] G. Thrivikraman, A. Athirasala, R. Gordon, L. Zhang, R. Bergan, D. R. Keene, J. M. Jones, H. Xie, Z. Chen, J. Tao, et al., Nat. Commun. 2019, 10, DOI 10.1038/s41467-01911455-8.

[27] Y. V. Shih, S. Varghese, Biomaterials 2019, 198, 107.

[28] A. M. Hughes, A. D. Kolb, A. B. Shupp, K. M. Shine, K. M. Bussard, Cancers (Basel). 
2021, 13, 1 .

[29] S. Ji, M. Guvendiren, Front. Bioeng. Biotechnol. 2017, 5, 1.

[30] H. Bahmaee, R. Owen, L. Boyle, C. M. Perrault, A. A. Garcia-Granada, G. C. Reilly, F. Claeyssens, Front. Bioeng. Biotechnol. 2020, 8, 1.

[31] P. Ribeiro, L. Leitão, A. C. Monteiro, A. Bortolin, B. Moura, M. Lamghari, E. Neto, Semin. Cell Dev. Biol. 2021, 112, 27.

[32] A. Mansoorifar, R. Gordon, R. C. Bergan, L. E. Bertassoni, Adv. Funct. Mater. 2021, 31, DOI 10.1002/adfm.202006796.

[33] A. Oryan, S. Alidadi, A. Moshiri, N. Maffulli, J. Orthop. Surg. Res. 2014, 9, 1.

[34] V. Herber, B. Okutan, G. Antonoglou, N. G. Sommer, M. Payer, J. Clin. Med. 2021, 10, DOI 10.3390/jcm10091842.

[35] B. Ferrigno, R. Bordett, N. Duraisamy, J. Moskow, M. R. Arul, S. Rudraiah, S. P. Nukavarapu, A. T. Vella, S. G. Kumbar, Bioact. Mater. 2020, 5, 468.

[36] M. Rahman, N. K. Dutta, N. Roy Choudhury, Front. Bioeng. Biotechnol. 2020, 8, 564. 
Chapter 8

Statement of impact 
To appreciate the scientific impact of the work presented in this thesis it is first important to recognize the field in which it is positioned.. Like the majority of biomedical research, it sits at the intersection of disciplines. Starting from fundamental to more applied topics, the studies presented here have aspects of biomineralization, materials science, and regenerative medicine. Inspiration for the use of biomineralized collagen was born out of discoveries made in the field of biomineralization, with its studies concerning processes of natural mineralization of biological tissues (bone in particular), but its contribution fits more completely under the umbrella of regenerative medicine. More specifically, this work contributes to the existing knowledge of biomimetic materials and their applications as in vitro models to study (patho)physiological processes and as clinical products. The main contribution to the field of biomimetic materials comes from the results describing interactions between osteoclasts and biomineralized collagen, presented in Chapter 3 and Chapter 4. By showing that osteoclast resorption of biomineralized collagen is possible, we add to the evidence that this material can indeed be considered one of the closest lab-made analogs for bone microenvironment. Osteogenic and osteoclast-related gene expression of hMSCs, explored in Chapter 5, further adds to this evidence.

Above everything else, the results and discussions presented in this thesis are relevant to the scientific community concerned with mimicking the bone microenvironment. On the one hand, they are useful for the continued development of bone graft substitutes, and on the other hand, as mentioned in Chapter 7, for preclinical studies of bone (patho)physiology and drug testing, the value of which depends on biomaterials that adequately resemble the native tissue.

Estimating the societal impact of pre-clinical in-vitro studies, like the ones presented in this thesis, is far more difficult, and in my opinion, only a step short of clairvoyance. In fact, currently, there is no direct, appreciable societal impact from the research presented in this thesis. Nonetheless, a few considerations can be made regarding its potential future application.

The contributions of this thesis to the understanding of biomineralized collagen as a biomaterial of choice for mimicking the microenvironment of bone may be valuable to biomedical companies active in the field of medical devices and regenerative medicine products, such as companies that develop bone graft substitute materials. A prospective market study from February 2021 estimates the global bone graft and 
substitutes market at USD 2.78 billion (2020), with a compound annual growth rate estimated around 5.8\% until 2028.[1] Companies such as DePuy Synthes, Stryker, Medtronic, Zimmer Biomet, Integra Orthobiologics, among others, all develop some form of synthetic bone graft substitute, usually comprising a biomimetic composition of calcium phosphate, collagen, or both.[2,3] The market share of synthetic graft substitutes (as opposed to human or animal derived products) has been increasing in the past, and is projected to grow in the coming years. In addition, synthetic bone graft subtitutes have become increasingly more biomimetic, with composite formulations (collagen-CaP) replacing single-component materials, sometimes even with biological growth factors added. However, so far, no commercial product is composed of collagen with intrafibrillar mineral, such as the one investigated in this thesis.

As mentioned in Chapter 1, in many clinical cases, bone defects are large and complex, and require grafting to aid its regeneration. As the population in developed countries skews older, the incidence of situations requiring a bone graft or a bone graft substitute increases, and so does the need for alternatives to the current standard of care (using the patient's own bone tissue).

The road that starts with fundamental discoveries of bone biomineralization processes is long and winding, passing by material design, optimization for regenerative strategies, and many other stops, before (potentially) reaching society at large, in the form of new and improved solutions to clinical challenges. The contributions made to science along that road stand on their own feet, even if the ultimate goal of a society-benefitting product remains elusive. 


\section{References}

[1] Grand View Research, “Bone Graft And Substitutes Market Size, Share \& Trends Analysis Report By Material Type (Allograft, Synthetic), By Application (Craniomaxillofacial, Dental, Foot \& Ankle), By Region, And Segment Forecasts, 2021 - 2028," can be found under https://www.grandviewresearch.com/industryanalysis/bone-grafts-substitutes-market, 2021.

[2] A. Oryan, S. Alidadi, A. Moshiri, N. Maffulli, J. Orthop. Surg. Res. 2014, 9, 1.

[3] A. Bow, D. E. Anderson, M. Dhar, Drug Metab. Rev. 2019, 51, 533. 


\section{Summary}

Synthetic bone graft substitutes are used in the clinic as alternatives to natural bone grafts, for repairing large bone defects. Currently, large, clinically challenging bone defects are predominantly treated by bone grafts form the patient undergoing surgery (autograft), or from a donor (allograft). Both solutions have inherent drawbacks, such as scarcity and complications associated with donor site surgery. Moreover, in the case of allografts, a risk of immune reaction exists. Synthetic bone graft substitutes avoid these drawbacks, and their continued development suggests that they will in the future replace autografts as the standard of care for regeneration of large bone defects. Most commercially available synthetic bone graft substitutes are composed of calcium phosphates and/or collagen, the two main components of the extracellular matrix (ECM) of bone. Biomimicry in general has been a common guiding principle behind biomaterials design for use as bone graft substitutes, as similarity with the native tissue underpins their performance. As reviewed in Chapter 2, the state-of-the-art bone biomimetic material is biomineralized collagen, recreating both the composition and structural architecture of bone's ECM, especially at the sub-micron scale. This thesis explores the potential of biomineralized collagen in bone regeneration and related regenerative medicine applications, by studying its interactions with two cell types from the bone environment, osteoclasts, and human mesenchymal stromal cells (hMSCs).

The formation and resorptive activity of osteoclasts on biomineralized collagen membranes were investigated in Chapter 3. Human monocytes/macrophages from peripheral blood were stimulated to differentiate into osteoclasts on collagen membranes with and without intrafibrillar mineral, as well as on cortical bone slices. Formation of osteoclasts occurred similarly on all substrates, but actin rings were observed only on the cortical bone control. Resorption pits and higher concentration of calcium ions in the cell culture medium confirmed that osteoclasts were actively resorbing cortical bone slices. In contrast, biomineralized and pure collagen membranes were not resorbed, likely due to the mesh-like surface structure of these substrates, accompanying mechanical properties, and possibly also due to a lack of RGD ligands to the vitronectin receptor. In the follow-up study, a calcium phosphate layer containing cobalt was added to the biomineralized collagen membranes (Chapter 4). As a result, two modifications of the membrane were achieved: an increase in mineral content of $\approx 4 \%(\mathrm{w} / \mathrm{w})$ and introduction of cobalt ions, previously shown to stimulate resorption by osteoclasts. Because of the additional mineral, the 
stiffness of the coated biomineralized collagen membranes increased from 1 to 10 $\mathrm{MPa}$, and the cobalt ions were slowly released in cell culture medium, reaching a concentration of $7 \mathrm{nM}$ after 2 days. Osteoclasts with actin rings were sporadically observed on the coated membranes, as well as the corresponding resorption lacunae. Moreover, a small increase in calcium ion concentration in cell culture medium was measured. It was suggested that the increase in membrane stiffness, as well as the presence of cobalt ions, resulted in osteoclasts being able to resorb the coated biomineralized collagen membrane, though to a limited extent, unlike biomineralized collagen membrane without additional calcium phosphate coating. Resorption by osteoclasts is one half of the process of bone remodeling, while the other half includes bone deposition by osteoblasts, which differentiate from progenitors residing in the mesenchymal stromal cell pool. To address this second part, in Chapter 5 the extent to which biomineralized collagen triggers osteogenic and osteoclast-related gene expression in hMSCs was investigated. Markers of osteogenic differentiation RUNX2, SPP1, ENPP1, and OCN showed increased expression after 3 days, and COL1A1 after 14 days of cell culture on biomineralized collagen as compared to culture on tissue culture plastic. OPG expression was upregulated in cells cultured on biomineralized collagen, and RANK-L was below detection limit, resulting in a low RANK-L/OPG ratio, which is indicative of a resorption-inhibiting hMSC phenotype. Overall, the results obtained showed that biomineralized collagen supports hMSCs growth and osteogenic differentiation, and could play a role in modulating the (pre)osteoblast/osteoclast communication. Chapter 6 offers a description of some optimization experiments and technical considerations, which may be useful for replication of the experiments performed in this thesis, as well as guidelines for future studies.

To conclude, this thesis illustrates the potential applicability of biomineralized collagen in regenerative medicine applications that require the micro-environment of bone to be mimicked. It does so by exploring the interactions between biomineralized collagen and the two fundamental cellular components of the bone environment, namely osteoclasts and hMSCs, and lays the ground for future improvements on this promising biomaterial. 


\section{Samenvatting}

Grote, complexe, beschadigingen van bot worden voornamelijk behandeld door middel van transplantatie van bot van de patiënt zelf, of van een donor. Beide oplossingen hebben belangrijke nadelen, zoals een beperkte hoeveelheid beschikbaar bot, complicaties van de benodigde ingreep en, in het geval van donormateriaal, de kans op afstoting van het getransplanteerde bot. Om deze nadelen te adresseren worden synthetische botvervangers in de kliniek gebruikt als alternatief voor natuurlijke bot transplantaties. De continue ontwikkeling van synthetische materialen suggereert dat synthetische botvervangers uiteindelijk de standaard behandeling van grote botdefecten zullen worden.

De meeste commercieel beschikbare synthetische botvervangers zijn gemaakt van calciumfosfaten en/of collageen, de twee hoofdcomponenten van de extracellulaire matrix (ECM) van bot. Naast de chemische samenstelling speelt ook de natuurlijke structuur van bot een belangrijke rol. Deze structuur wordt hierom bij deze synthetische botvervangers vaak nagebootst. Zoals samengevat in het literatuuronderzoek van Hoofdstuk 2, is de huidige state-of-the-art binnen de synthetische botvervangers biologisch gemineraliseerd collageen, dat zowel de structuur als de samenstelling van de ECM van bot imiteert, voornamelijk op submicrometer schaal. Dit proefschrift onderzoekt de potentie van biologisch gemineraliseerd collageen als botvervanger, door de interacties met twee menselijke botceltypen te bestuderen: osteoclasten en mesenchymale stromale cellen (MSC's). De ontwikkeling en resorptie-activiteit van osteoclasten op biologisch gemineraliseerde collageen membranen zijn onderzocht in Hoofdstuk 3. Menselijke monocyten/macrofagen uit het perifere bloed werden gestimuleerd om te differentiëren tot osteoclasten op membranen van collageen met en zonder intrafibrillair mineraal, en op corticale bot schijfjes. Osteoclasten werden gevormd op alle substraten in vergelijkbare hoeveelheden, maar actine ringen werden alleen geobserveerd op de corticale bot controle. Resorptiekuiltjes en een hogere concentratie calcium ionen in het celkweek medium bevestigden dat de osteoclasten actief de corticale bot schijfjes resorbeerden. Biologisch gemineraliseerd collageen membranen en collageen membranen zonder mineraal werden niet geresorbeerd, waarschijnlijk door de poreuze, gaasachtige structuur van het oppervlakte van deze substraten. De mechanische eigenschappen en de afwezigheid van RGD liganden van de vitronectine receptor speelden mogelijk ook een. In de vervolgstudie is er een calciumfosfaat laag met kobalt toegevoegd aan de gemineraliseerde collageen 
membranen (Hoofdstuk 4). Hierdoor zijn er twee veranderingen aan de membranen bewerkstelligd: een toename in de hoeveelheid mineraal van ongeveer 4 gewichtsprocent en de introductie van kobalt ionen, waarvoor voorheen is bewezen resorptie door osteoclasten te stimuleren. Door de toename in de hoeveelheid mineraal nam ook de stijfheid van de membranen toe van $1 \mathrm{MPa}$ naar $10 \mathrm{MPa}$. De kobalt ionen kwamen langzaam vrij in het celkweek medium, tot een concentratie van $7 \mathrm{nM}$ na 2 dagen. Op deze membranen met extra calciumfosfaat coating werden sporadisch osteoclasten met actine ringen waargenomen, en de bijbehorende resorptiekuiltjes. Er werd ook een kleine toename in de concentratie calcium ionen waargenomen in het celkweek medium. Deze resultaten suggereren dat de toename in de stijfheid van de membranen en de aanwezigheid van kobalt ionen resulteerde in resorptie van het biologisch gemineraliseerd collageen door osteoclasten, al was het in kleine hoeveelheden.

Resorptie door osteoclasten is de helft van het botremodeleringproces. De andere helft is de aanmaak van nieuw bot door osteoblasten, die differentiëren uit voorgangers in de mesenchymale stroma. Om dit tweede deel te onderzoeken is in Hoofdstuk 5 het effect van biologisch gemineraliseerd collageen op osteoblast en osteoclast gerelateerde genexpressie in MSC's bestudeerd. Markers voor osteoblast differentiatie RUNX2, SPP1, ENPP1 en OCN kwamen meer tot expressie na 3 dagen, en COL1A1 na 14 dagen celkweek op biologisch gemineraliseerd collageen, in vergelijking met een plastic celkweek oppervlakte. OPG expressie was hoger in cellen die werden gekweekt op biologisch gemineraliseerd collageen en RANK-L was onder de detectielimiet, wat resulteerde in een lage RANK-L/OPG ratio, een indicatie van een resorptie-remmend MSC fenotype. Deze resultaten laten zien dat gemineraliseerd collageen de groei en differentiatie van MSC's ondersteunt en mogelijk een rol speelt in het reguleren van (pre)osteoblast/osteoclast communicatie. In Hoofdstuk 6 staan een aantal optimalisatie experimenten en technische details beschreven, die kunnen helpen bij het reproduceren van de experimenten in dit proefschrift, en het uitvoeren van nieuwe studies.

Samengevat beschrijft dit proefschrift de mogelijke toepassing van biologisch gemineraliseerd collageen in de regeneratieve geneeskunde waarbij de microomgeving van bot nagebootst moet worden. Dit werd onderzocht door de interacties te bestuderen tussen gemineraliseerd collageen en de twee fundamentele cellulaire componenten van bot, osteoclasten en MSC's. Met dit onderzoek wordt een basis gelegd voor toekomstige verbeteringen aan dit veelbelovende materiaal. 


\section{Acknowledgements}

Come now, sit! And by this fire rest;

Bread butter and beer will abound

Before this tale travels around.

Much mirth and merry, maybe a jest,

Preclude our serious quest.

This book you have read, was by

Valiant effort to your hands brought.

Mistake it not as if by one wrought

For however deft, no single hand could

Such portentous task complete,

Lest all his sanity deplete.

Yet here it stands for all to see,

The work of this once knight-to-be.

How come you ask, who was that helped,

How many hands hide behind his own?

The quest now clear, our sights alight

In the shore where those live bright

That this knight helped and made worthy.

Were that I could name them all without

A fault!

What then makes a knight? (or a scientist!)

If not his mentors to inspire

And in their wisdom share

Ideas that convention defy.

If not his teachers and spirited

Challengers that barriers and hurdles

Along the way erected.

If not his fellow questers

That laughing through sorrow,

Steel-bonded brotherhood build. 
If not his squires that unseen

Countless trivial task completed

And less the worry for the one ahead.

If not his family,

That by being in body and spirit

A remembrance that not skill alone

Guides a hand, but also kindness

For others yes, but also, for himself.

Of them all this Knight is made

And for them all this poem,

I, indebted,

Offer. 


\section{List of publications}

Pertaining to this thesis

Daniel de Melo Pereira and Pamela Habibović. Biomineralization-inspired material design for bone regeneration. Advanced Healthcare Materials, 2018, 1800700, 1-18

Daniel de Melo Pereira, Maria Eischen-Loges, Zeinab Tahmasebi Birgani and Pamela Habibović. Proliferation and osteogenic differentiation of hMSCs on biomineralized collagen. Frontiers in Bioengineering and Biotechnology, 2020, $8: 554565,1-16$

Daniel de Melo Pereira, Matthias Schumacher and Pamela Habibović. Cobaltcontaining calcium phosphate induces resorption of biomineralized collagen by human osteoclasts. Biomaterials Research, 2021, 25:6, 1-13

Daniel de Melo Pereira, Noel Davison and Pamela Habibović. Human osteoclast formation and resorptive function on biomineralized collagen. Bioactive Materials, 2021, 10.1016/j.bioactmat.2021.06.036

\section{$\underline{\text { Others }}$}

Ziryan Othman, Hugo Fernandes, Arjan Groot, Theo M. Luiderd, Alessandro Alcinesio, Daniel de Melo Pereira, Alexander P. M. Guttenplan, Huipin Yuan, Pamela Habibović. The role of ENPP1/PC-1 in osteoinduction by calcium phosphate ceramics. Biomaterials, 2019, 210, 12-24.

Lanying Sun, Daniel Pereira, Qibao Wang, David Baião Barata, Roman Truckenmüller, Zhaoyuan Li, Xin Xu, Pamela Habibović. Controlling Growth and Osteogenic Differentiation of Osteoblasts on Microgrooved Polystyrene Surfaces. PLOS One, 2016, 11(8): e0161466

Lanying Sun, Charlène B. Danoux, Qibao Wang, Daniel Pereira, David Barata, Jingwei Zhan, Vanessa LaPointe, Roman Truckenmüller, Chongyun Bao, Xin Xu, Pamela Habibović. Independent effects of the chemical and microstructural surface properties of polymer/ceramic composites on proliferation and osteogenic differentiation of human MSCs. Acta Biomaterialia, 2016, 42, 364-377. 
D. Barata, A. Resmini, D. Pereira, S. A. Veldhuis, C. A. van Blitterswijk, J. E. ten Elshof and P. Habibović. Surface micropatterning with zirconia and calcium phosphate ceramics by micromoulding in capillaries. Journal of Materials Chemistry B, 2016, 4, 1044-1055.

Charlène Danoux, Daniel Pereira, Nicola Döbelin, Christoph Stähli, Jake Barralet, Clemens van Blitterswijk, Pamela Habibović. The Effects of Crystal Phase and Particle Morphology of Calcium Phosphates on Proliferation and Differentiation of Human Mesenchymal Stromal Cells. Advanced Healthcare Materials, 2016, 5, 1775-1785. 
